\title{
Arithmetic and analytical aspects of Siegel modular forms
}

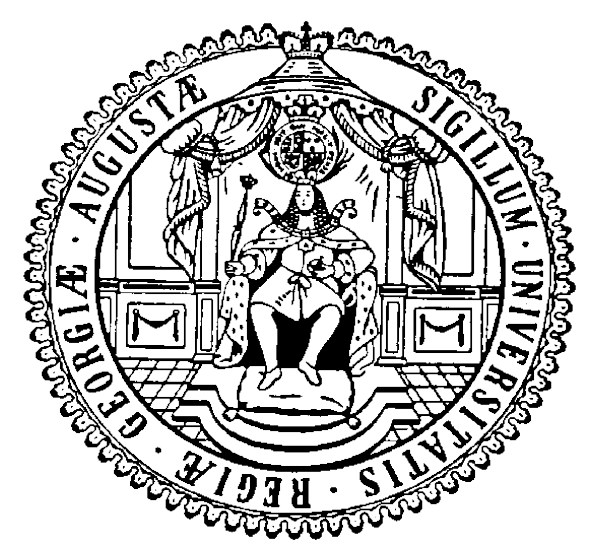

Dissertation

zur Erlangung des mathematisch-naturwissenschaflichen Doktorgrades

"Doctor rerum naturalium"

der Georg-August Universität Göttingen

im Promotionsstudiengang "Mathmatical Sciences (Ph.D)"

der Georg-August University School of Science (GAUSS)

vorgelegt von

Fabian Christoph Lutz Waibel

aus Wiesbaden

Göttingen, 2020 
Betreuungsausschuss:

Prof. Dr. Valentin Blomer,

Mathematisches Institut, Georg-August Universität Göttingen bis 30.09.2019

Mathematisches Institut, Rheinische Friedrich-Wilhelms-Universität Bonn ab 01.10.2019

Prof. Dr. Jörg Brüdern

Mathematisches Institut, Georg-August Universität Göttingen

$\underline{\text { Mitglieder der Prüfungskommission }}$

Referent: Prof. Dr. Valentin Blomer

Korreferent: Prof. Dr. Jörg Brüdern

Weitere Mitglieder der Prüfungskommission (alle Georg-August Universität Göttingen)

Prof. Dr. Preda Mihăilescu, Mathematisches Institut

Prof. Dr. Gerlind Plonka-Hoch, Institut für Numerische und Angewandte Mathematik

Jun.-Prof. Dr. Daniel Rudolf, Institut für Mathematische Stochastik

Prof. Dr. Chenchang Zhu, Mathematisches Institut

Tag der mündlichen Prüfung: 25.06.2020 


\section{Acknowledgement.}

First and foremost, I am very grateful to Prof. Dr. Valentin Blomer for raising my interest in modular forms, his continuous support during my master and $\mathrm{PhD}$ thesis and his valuable feedback. Furthermore, I sincerely thank Prof. Dr. Brüdern for his counsel, constant support and his open-door policy. Moreover, I thank my girlfriend Bri, my office mates Burkhard and Rebecca as well as Max for advice, emotional support and consistent encouragement. Last but not least I would like to thank my parents for their counsel and support. 


\section{Contents}

1 Introduction 5

1.1 Moments of Spinor $L$-functions . . . . . . . . . . . . . . . . . 9

1.2 Representation of integers by quadratic forms . . . . . . . . . . 12

1.3 Representation of binary quadratic forms by quadratic forms . . . . 15

$\begin{array}{lll}2 & \text { Moments of Spinor } L \text {-Functions } & 19\end{array}$

2.1 Representations, Newform Theory and Saito-Kurokawa Lifts. . . . . 20

2.2 The Spinor $L$-Function and Böcherer's conjecture for oldforms. . . . 24

2.3 Kitaoka-Petersson formula $\ldots \ldots \ldots \ldots \ldots \ldots \ldots$

2.4 Symplectic Kloosterman sums . . . . . . . . . . . . . . . . 33

2.5 Proof of Theorem $1 \ldots \ldots \ldots \ldots \ldots \ldots$

3 Theta series of degree one $\quad 42$

4 Theta series and Klingen-Eisenstein series of degree two 58

4.1 Siegel modular forms and the space of Eisenstein series . . . . . . . 58

4.1 .1 Eisenstein series $\ldots \ldots \ldots \ldots \ldots \ldots \ldots \ldots$

4.1 .2 Theta series and representation of quadratic forms . . . . . . 64

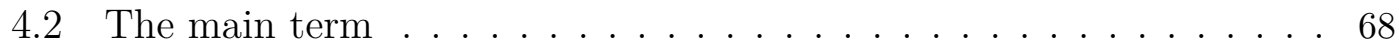

4.3 The error term for prime level . . . . . . . . . . . . . . . . . 71

4.3 .1 Inner product of theta series . . . . . . . . . . . . . . 72

4.3.2 Decomposition of matrices in $\mathrm{Sp}_{4}(\mathbb{Z})$ and $\Gamma_{0}^{(2)}(N) \ldots \ldots .78$

4.3.3 Bounds for Fourier coefficients of Klingen-Eisenstein series. . 88

4.3 .4 Bounds for Fourier coefficients of theta series . . . . . . . . 106

4.4 Extensions . . . . . . . . . . . . . . . . . . . . . . . . . . . . . 113

$4.4 .1 \quad$ Squarefree level . . . . . . . . . . . . . . . . . . . . . . . . . 114

4.4 .2 Quadratic nebentypus . . . . . . . . . . . . . . . 125 


\section{Introduction}

Modular forms first appeared in the theory of elliptic integrals and quadratic forms in the nineteenth century. Since then they occupy a central role in number theory and have numerous applications not only within the scope of number theory, but also in other fields of mathematics, or even in string theory. A prominent example is Taylor and Wiles's proof of Fermat's Last Theorem.

In the classical sense, modular forms are complex analytical functions $f: \mathbb{H}=$ $\{z \in \mathbb{C} \mid \operatorname{Im}(z)>0\} \rightarrow \mathbb{C}$ with a certain transformation behavior under a subgroup $\Gamma$ of $\mathrm{SL}_{2}(\mathbb{Z})$ that is given by

$$
f(\gamma z)=f\left(\frac{a z+b}{c z+d}\right)=\chi(\gamma)(c z+d)^{k} f(z), \quad \gamma=\left(\begin{array}{ll}
a & b \\
c & d
\end{array}\right) \in \Gamma
$$

where $\chi$ is a character on $\Gamma$ and $k$ is the weight of $f$. Furthermore, we require that $f$ satisfies the growth condition $f(x+i y) \ll y^{-\nu}+y^{\nu}$ for some fixed $\nu$.

A classic example is the theta series. We consider a positive, integral quadratic form

$$
q\left(x_{1}, \ldots, x_{m}\right)=\sum_{i} q_{i i} x_{i}^{2}+\sum_{i \neq j} q_{i j} x_{i} x_{j}
$$

with corresponding matrix $Q=\left(q_{i j}\right)$ and let

$$
\theta(Q, z)=\sum_{x \in \mathbb{Z}^{m}} e\left(\frac{1}{2} q(x) z\right)
$$

for $e(x):=\exp (2 \pi i x)$. Let $N$ denote the level of $Q$, which is the smallest integer such that $N Q^{-1}$ is integral and has even diagonal entries. Then, $\theta(Q, z)$ is a modular form of weight $\frac{m}{2}$ with respect to the group

$$
\Gamma_{0}(N)=\left\{\left(\begin{array}{ll}
a & b \\
c & d
\end{array}\right) \in \mathrm{SL}_{2}(\mathbb{Z}) \mid c \equiv 0(\bmod N)\right\}
$$

and the quadratic character $\chi\left(\begin{array}{ll}a & b \\ c & d\end{array}\right)=\left(\frac{(-1)^{\frac{m}{2}} \operatorname{det} Q}{d}\right)$ if $m$ is even and $\left(\frac{(-1)^{\frac{m-1}{2}} c \operatorname{det} Q}{d}\right)$ if $m$ is odd. Since $\theta(Q, z)=\theta(Q, z+1)$ we obtain a Fourier extension

$$
\theta(Q, z)=\sum_{n \geqslant 0} r(n, Q) e(n z)
$$

where $r(n, Q)=\left\{x \in \mathbb{Z}^{m} \mid q(x)=n\right\}$ counts the number of representations of $n$ by the quadratic form $q$. Now, one of the main advantages of modular forms comes into play. The space of modular forms of given weight $k$ on $\Gamma$ is finite and algorithmically computable. In this way, we can approximate $\theta(Q, z)$ by another, 
well-understood modular form and eventually obtain an asymptotic formula for $r(Q, n)$.

The theory of modular forms took a giant leap forward in 1937 when Hecke [25] introduced averaging operators that give essential arithmetic information on the underlying modular form. As a consequence, Hecke could interpret the Fourier coefficients of a $\mathrm{SL}_{2}(\mathbb{Z})$ cusp form ${ }^{1}$, that is a simultaneous eigenform of all Hecke operators, as eigenvalues of these operators. Later, this theory was extended to $\Gamma_{0}(N)$ and characters by Atkin and Lehner [3]. For a "Hecke eigenform" of $\Gamma_{0}(N)$, the Dirichlet series $L(f, s)=\sum_{n \geqslant 1} a(n) n^{-s}$ can be expressed as an Euler product

$$
\prod_{p}\left(1-\lambda(p) p^{-s}+\chi(p) p^{k-1-2 s}\right)
$$

where $k$ is the weight of $f$ and $\chi$ its character. Using Mellin inversion, Hecke proved that $L(f, s)$ can be continued to a holomorphic function on the whole complex plane and satisfies a functional equation relating $L(f, s)$ to $L(g, k-s)$ where $g(z)=z^{k} f\left(\frac{-1}{n z}\right)$. The work of Hecke was later simplified by his student Petersson who introduced an inner product with respect to which Hecke's operators are normal. A direct consequence of this property is the existence of a basis of Hecke eigenforms for the space of cusp forms.

Around the same time another direction was pursued by Siegel [55]. Inspired by the quantitative theory of representing quadratic forms by quadratic forms, he generalized the notion of modular forms to matrices of higher dimension. For two positive integral quadratic forms in $m$ and $n$ variables given by matrices $Q$ and $2 T$ he studied the number of representations

$$
r(Q, T))=\#\left\{X \in M_{m, n}(\mathbb{Z}) \mid \frac{1}{2} X^{T} Q X=T\right\} .
$$

His starting point was a generalized upper half space

$$
\mathbb{H}_{n}=\left\{Z=X+i Y \in M_{n}(\mathbb{C}) \mid Z^{T}=Z, Y>0\right\}
$$

and a generalized theta series

$$
\theta(Q, Z)=\sum_{X \in M_{m, n}(\mathbb{Z})} e\left(\frac{1}{2} X^{T} Q X Z\right), \quad Z \in \mathbb{H}_{n} .
$$

Just as its one-dimensional counterpart, it satisfies $\theta\left(Q,-Z^{-1}\right)=(\operatorname{det} Q)^{-1} \theta\left(Q^{-1}, Z\right)$. Furthermore, as $\theta(Q, Z)=\theta(Q, Z+S)$ for any symmetric, integer $n \times n$ matrix $S$, this series admits a Fourier expansion

$$
\theta(Q, Z)=\sum_{T \in \mathcal{S}_{n}} r(Q, T) e(\operatorname{tr} T Z)
$$

\footnotetext{
${ }^{1} \mathrm{~A}$ cusp form is a modular form that satisfies $f(x+i y) \ll y^{-\frac{k}{2}}$ for $x+i y \in \mathbb{H}$. In terms of Fourier expansions this means that the zeroth term $a(0)$ vanishes.
} 
where $\mathcal{S}_{n}$ denotes all $n \times n$ positive semi-definite, half-integer matrices with even diagonal. With this in mind, Siegel considered holomorphic functions $F: \mathbb{H}_{n} \rightarrow \mathbb{C}$ that satisfy

$$
F(M Z):=F\left((A Z+B)(C Z+D)^{-1}\right)=(\operatorname{det}(C Z+D))^{k} \chi(M) F(Z)
$$

for $M=\left(\begin{array}{ll}A & B \\ C & D\end{array}\right)$ in a subgroup $\Gamma$ of $\operatorname{Sp}_{2 n}(\mathbb{Z})$ and a character $\chi$ on $\Gamma$. Recall that

$$
\mathrm{Sp}_{2 n}(\mathbb{Z})=\left\{M \in \mathrm{SL}_{2 n}(\mathbb{Z}) \mid M^{T} J_{2 n} M=J_{2 n}\right\} \quad \text { for } \quad J_{2 n}=\left(\begin{array}{cc}
-I_{n} \\
I_{n}
\end{array}\right) .
$$

For $n \geqslant 2$ such a function is automatically holomorphic at the cusps as shown later by Koecher [39]. Nowadays, they are called Siegel modular forms and for example, $\theta(Q, Z)$ transforms with respect to the group

$$
\Gamma_{0}^{(n)}(N)=\left\{\left(\begin{array}{ll}
A & B \\
C & D
\end{array}\right) \in \operatorname{Sp}_{2 n}(\mathbb{Z}) \mid C \equiv 0(\bmod N)\right\}
$$

and the quadratic character

$$
\chi(M)=\left(\frac{(-1)^{k} \operatorname{det} Q}{\operatorname{det} D}\right) .
$$

As we have already seen for $\theta(Q, Z)$, a Siegel modular form $F$ with respect to $\Gamma_{0}^{(n)}(N)$ possesses a Fourier expansion $F(Z)=\sum_{T \in \mathcal{S}_{n}} A(T) e(\operatorname{tr} T Z)$. We say that $F$ is a cusp form, if $F$ vanishes at all cusps. In terms of Fourier expansion, this implies that the coefficients (at every cusp) vanish unless $T$ is positive definite.

In comparison to classical modular forms, both the theory of Hecke operators and $L$-functions become substantially more complex. For $n=2$ there still exists a basis of Hecke eigenform for the space of cusp forms. However, it is no longer possible to express every Fourier coefficients in terms of Hecke eigenvalues. In addition, the Fourier coefficients are attached to matrices instead of integers which makes embedding them in an $L$-function cumbersome. As a consequence, $L$-functions are defined for $n \geqslant 2$ as an Euler product rather than a Dirichlet series over Fourier coefficients.

Nonetheless, the complexity of Hecke relations makes it difficult to define an $L$-function using Hecke eigenvalues. A key difference to classical modular forms is that Hecke operators at primes $T(p)$ are no longer sufficient to generate the associated Hecke algebra. A solution is to consider Satake parameters instead. These are tuples of $n+1$ complex numbers that are constructed using information about the Hecke algebra and are closely connected to the roots of the Hecke polynomial. For $n=1$, let $\beta_{0, p}, \beta_{1, p}$ denote the roots of the Hecke polynomial 
$1-\lambda(p) X+\chi(p) p^{k-1} X^{2}$, cf. (1). Then, the Satake parameters $\left(\alpha_{0, p}, \alpha_{1, p}\right)$ are given by $\alpha_{0, p}=\beta_{0, p}$ and $\alpha_{0, p} \alpha_{1, p}=\beta_{1, p}$. Thus, for $n=1$ the $L$-function is given by

$$
\prod_{p}\left(1-\alpha_{0, p} p^{-s}\right)^{-1}\left(1-\alpha_{0, p} \alpha_{1, p} p^{-s}\right)^{-1}
$$

We generalize the concept above to a Hecke eigenform $F$ (of level 1) with Satake parameters $\left(\alpha_{0, p}, \ldots, \alpha_{n+1, p}\right)$ by setting

$$
L(s, F)=\prod_{p}\left(1-\alpha_{0, p} p^{-s}\right)^{-1} \prod_{r=1}^{n} \sum_{1 \leqslant i_{1}<\ldots<i_{r} \leqslant r}\left(1-\alpha_{0, p} \alpha_{i_{1}, p} \ldots \alpha_{i_{r}, p} p^{-s}\right)^{-1} .
$$

This function is holomorphic for $\operatorname{Re}(s)$ sufficiently large and is called the spinor $L$-function. For $n=2$ Andrianov [1] showed that $L(s, F)$ can be meromorphically continued to the entire complex plane and proved a functional equation relating $L(s, F)$ to $L(1-s, F)$.

To evaluate $L$-functions, a standard technique is to consider families of $L$ functions and obtain statistical results on average. This way, many problems can be treated that are out of reach when specializing to a single L.function. A classic example for such a family is the set of characters for a given modulus. This approach enabled Dirichlet to link the problem of counting primes in arithmetic progression to $L$-functions of Dirichlet characters. By $L(1, \chi)>0$ for non-trivial $\chi$, it follows that there are infinitely many primes of the form $a+n d$ with $(a, d)=1$. More generally, the existence of a zero-free region of $L(s, \chi)$ allows us to estimate the distribution of primes and a larger width of the zero-free region implies more accurate results.

To study $L$-functions of modular forms on average, the usual approach is to sum over a suitable base of the underlying space of cusp forms and consider the first and second moment on the critical line. For the spinor $L$-function of degree two, these moments have been computed by Blomer [6] via a spectral summation formula in the case of large weight. For this purpose, the moments are twisted by a harmonic weight that - by a recent breakthrough - itself is the product of two central $L$-values. Thus, Blomer actually evaluates a fourth moment. As an application, various non-vanishing results for central $L$-values follow.

This dissertation focuses on the level aspect of Siegel modular forms of degree two with respect to $\Gamma_{0}^{(2)}(N)$. In the first part we compute a second moment of the spinor $L$-function for large prime levels. In the second part, we prove an asymptotic formula for the Fourier coefficients $r(Q, T)$ of the Siegel theta series for squarefree level. As a byproduct, we improve previous results for the Fourier coefficients $r(Q, n)$ of the classical theta series. All estimates are uniform in the level $N$, meaning that, for every term, we meticulously keep track of the dependence on $N$.

Before we go into detail on the specific problems, we present an application that shows the benefit of uniform bounds in the level. It is conjectured that every 
natural number $n \equiv 3(\bmod 8), 5 \nmid n$ is the sum of three squares of primes. By current methods this problem is out of reach, but results can be obtained for almost primes, cf. [5, Section 3.1] and [7]. The main tool is a powerful sieve that filters out the small primes. The implementation of this sieve requires a good estimate on the number of representations of $n=x_{1}^{2}+x_{2}^{2}+x_{3}^{2}$ with $x_{j}$ divisible by a not too large squarefree number. More precisely, we need an asymptotic formula for the number of representations

$$
n=d_{1}^{2} x_{1}^{2}+d_{2}^{2} x_{2}^{2}+d_{3}^{2} x_{3}^{2}, \quad d_{i} \text { squarefree }
$$

that is uniform with respect to the coefficients $d_{i}$, or in other words, the level of the quadratic form $d_{1}^{2} x_{1}^{2}+d_{2}^{2} x_{2}^{2}+d_{3}^{2} x_{3}^{2}$. Altogether, this approach from Blomer and Brüdern yields that every $n$ satisfying the congruence conditions above is represented by three squares of almost primes. To the authors' knowledge, the best result so far is the representation by almost primes that each have at most 106 prime divisors, cf. [13].

\subsection{Moments of Spinor $L$-functions}

For the analytical theory of modular forms on congruence subgroups in $\mathrm{GL}_{2}(\mathbb{Z})$, spectral summation formulas such as the Petersson formula are a basic tool. A primary component is a sum over Kloosterman sums and many applications rely on a careful estimation of the latter. For Siegel cusp forms, Kitaoka [30] introduced an analogue to Petersson's formula that was extended in [15] to include congruence subgroups. In this case, however, the off-diagonal terms are very complex and contain generalized Kloosterman sums that run over matrices in $\operatorname{Sp}_{4}(\mathbb{Z})$. So far, the literature on these sums is limited.

The aim of this part is to evaluate spectral averages of second moments of spinor $L$-functions for Siegel congruence groups of large prime level by means of the Kitaoka-Petersson formula. The core of this computation is the manipulation of symplectic Kloosterman sums which may be of independent interest.

To state our results, we fix some notation. Let $S_{k}^{(2)}(N)$ denote the space of Siegel cusp forms on $\Gamma_{0}^{(2)}(N)$ of weight $k$. For $F, G \in S_{k}^{(2)}(N)$, we define the (unnormalized) Petersson inner product by

$$
\langle F, G\rangle=\int_{\Gamma_{0}^{(2)}(N) \backslash \mathbb{H}_{2}} F(Z) \overline{G(Z)}(\operatorname{det} Y)^{k} \frac{d X d Y}{(\operatorname{det} Y)^{3}} .
$$

Any $F \in S_{k}^{(2)}(N)$ has a Fourier expansion

$$
F(Z)=\sum_{T \in \mathscr{S}} a_{F}(T)(\operatorname{det} T)^{\frac{k}{2}-\frac{3}{4}} e(\operatorname{tr}(T Z)),
$$

with Fourier coefficients $a_{F}(T)$, where $\mathscr{S}$ is the set of symmetric, positive definite, half integral matrices $T$ with integral diagonal entries. We choose an orthogonal 
basis $B_{k}^{(2)}(N)^{\text {new }}$ of newforms in the sense of [49] in $S_{k}^{(2)}(N)$ such that the adelization of each element generates an irreducible representation and for each prime $p \mid N, F$ is an eigenfunction of the $T_{2}(p)$ operator, cf. (14).

In the following, $N$ is prime and $N \equiv 3(\bmod 4) 2$ For $F \in B_{k}^{(2)}(N)^{\text {new }}$ of even weight $k$, we let $L(s, F)$ denote the spinor $L$-function, normalized so that its critical strip is $0<\Re s<1$. This is a degree $4 L$-function. Furthermore, we set

$$
w_{F, N}:=\frac{\pi^{1 / 2}}{4}(4 \pi)^{3-2 k} \Gamma(k-3 / 2) \Gamma(k-2) \frac{\left|a_{F}(I)\right|^{2}}{\langle F, F\rangle},
$$

where $I$ is the $2 \times 2$ identity matrix. These "harmonic" weights appear naturally in the Kitaoka-Petersson formula. Due to the non-normalization of the inner product they implicitly contain a factor of $\left[\operatorname{Sp}_{4}(\mathbb{Z}): \Gamma_{0}(N)\right]=N^{3}$ in the denominator. On average, they are of size $=N^{-3}$, i.e. it holds by [17, p. 37] that

$$
\sum_{F \in B_{k}^{(2)}(N)^{\text {new }}} w_{F, N}=1+\mathcal{O}\left(N^{-1} k^{-2 / 3}\right)
$$

In addition, the weights $w_{F, N}$ are related to central values of $L$-functions. This remarkable conjecture is due to Böcherer and was recently proven in [20, Theorem 2 \& Remark 6]. Let $S_{k}^{(2)}(N)^{\text {new, T }}$ denote the space of newforms orthogonal to Saito-Kurokawa lifts. For $F \in S_{k}^{(2)}(N)^{\text {new,T }}$ that satisfy $w_{F, N} \neq 0$, we have by [17, Theorem 1.12] that

$$
w_{F, N}=c \frac{L(1 / 2, F) L\left(1 / 2, F \times \chi_{-4}\right)}{N^{3} L\left(1, \pi_{F}, \mathrm{Ad}\right)},
$$

where $L\left(s, \pi_{F}, \mathrm{Ad}\right)$ denotes the degree 10 adjoint $L$-function and $c$ is an explicit constant depending on $F$, see Lemma 8 .

Let $q_{1}, q_{2}$ be two fixed coprime fundamental discriminants (possibly 1 ) and denote by $\chi_{q_{1}}$ the character which maps $x$ to the Kronecker symbol $\left(\frac{q_{1}}{x}\right)$.

Theorem 1. For $k \geqslant 10$ and a prime $N \equiv 3(\bmod 4)$ it holds that

$$
\sum_{F \in B_{k}^{(2)}(N)^{\text {new }}} w_{F, N} L\left(1 / 2, F \times \chi_{q_{1}} \overline{L\left(1 / 2, F \times \chi_{q_{2}}\right)}=\text { main term }+\mathcal{O}_{q_{1}, q_{2}, k}\left(N^{-\alpha+\epsilon}\right),\right.
$$

where the main term is the residue at $s=t=0$ of the expression (53) and $\alpha=\frac{1}{2}$ for $k \geqslant 20$ and $\alpha=\frac{k-9}{k+1}$ for $k \leqslant 18$. In particular, if $q_{1}=q_{2}=1$, the main term equals

$$
\frac{4}{3} L\left(1, \chi_{-4}\right)^{2} P_{1}(\log N)
$$

${ }^{2}$ The assumptions $N \equiv 3(\bmod 4)$ is required for local non-archimedean computations in [17] and Section 2.2 The cited results from [17] hold for squarefree $N$ with prime divisors $p \equiv 3(\bmod 4)$. 
for a certain monic polynomial $P_{1}$ of degree 3 depending on $k$.

If $q_{1}, q_{2} \in\{1,-4\}$, the main term equals

$$
2 L\left(1, \chi_{-4}\right)^{2} P_{2}(\log N)
$$

for a certain monic polynomial $P_{2}$ of degree 2 depending on $q_{1}, q_{2}$ and $k$.

If $q_{1}, q_{2}$ are two coprime integers different from 1 and -4 , the main term equals

$$
4 L\left(1, \chi_{q_{1}}\right) L\left(1, \chi_{-4 q_{1}}\right) L\left(1, \chi_{q_{2}}\right) L\left(1, \chi_{-4 q_{2}}\right) L\left(1, \chi_{q_{1} q_{2}}\right) .
$$

In view of Böcherer's conjecture, Theorem 1 even evaluates a fourth moment of central values and a degree 16 L-function.

For large weights and the full modular group, i.e. $N=1$, Blomer [6] shows a very similar result and the proof of Theorem 1 is based on his work. While obtaining a uniform estimate in weight $k$ and level $N$ is principally possible, this requires however a Petersson formula for newforms. In the GL(2) case, such a formula is well-known and derived by first constructing an explicit orthogonal basis of oldforms and then, applying Möbius inversion to sieve these forms out, cf. [42].

The main difficulty of proving Theorem 1 is treating the off-diagonal contribution in the Kitaoka-Petersson formula. This term is a sum over Bessel functions and symplectic Kloosterman sums whose "moduli" run over integral 2-by-2 matrices with all entries divisible by $N$. Consequently, we decompose each Kloosterman sum into two parts, separating a Kloosterman sum of modulus $N I$ that is straightforward to handle. After applying Poisson summation, we see that the sum vanishes unless a specific congruence condition is fulfilled. In this way, only matrices in $\mathrm{GO}_{2}(\mathbb{Z})=\mathbb{R}_{>0} \cdot O(2) \cap \operatorname{Mat}_{2}(\mathbb{Z})$ survive as possible moduli for the remaining Kloosterman sums. This corresponds to the case of large weight in [6] and the remaining term can be computed in exactly the same way. In contrast to Blomer, who uses special features of Bessel functions, we manipulate symplectic exponential sums and evaluate congruences. Hence, this work can be seen as a non-archimedean version of [6], where the analysis of oscillatory integrals is replaced - in disguise - by its $p$-adic analogue.

The contribution of Saito-Kurokawa lifts to the left hand side of (8) is very small. If $f$ is the elliptic modular newform corresponding to the lift $F$, then $w_{F, N}$ is related to central $L$-values of $f$, i.e. by [17, Theorem 3.12] we have that

$$
w_{F, N}=\frac{3(2 \pi)^{7} \Gamma(2 k-4)}{N^{3} \Gamma(2 k-1)} \frac{L\left(1 / 2, f \times \chi_{-4}\right)}{L(3 / 2, f) L(1, f, \mathrm{Ad})} .
$$

Applying simply the convexity bound for central $L$-values, we see that the contribution of the $\mathcal{O}(N)$ Saito-Kurokawa lifts is $\mathcal{O}\left(N^{-5 / 4+\epsilon}\right)$.

Let $B_{k}^{(2)}(N)^{\text {new,T }}$ denote a basis of $S_{k}^{(2)}(N)^{\text {new,T }}$ with the same properties as in Theorem 1. By applying (6), Cauchy-Schwarz and (10), we get: ${ }^{3}$

\footnotetext{
${ }^{3}$ We use the superscript $\mathrm{T}$ for the space orthogonal to Saito-Kurokawa lifts since conjecturally the associated local representations are tempered everywhere.
} 
Corollary 2. For $k \geqslant 10$ and a sufficiently large prime $N \equiv 3(\bmod 4)$, it holds that

$$
\sum_{\substack{F \in B_{k}^{(2)}(N)^{\text {new }, \mathrm{T}} \\ w_{F, N} \neq 0}} \frac{1}{L\left(1, \pi_{f}, \mathrm{Ad}\right)} \gg \frac{N^{3}}{(\log N)^{2}} .
$$

In particular, if $L\left(1, \pi_{f}, \mathrm{Ad}\right)$ has no zeros in $|s-1| \ll N^{-\epsilon}$, then $N^{3-\epsilon}$ forms $F \in B_{k}^{(2)}(N)^{\text {new,T }}$ satisfy $w_{F, N} \neq 0$ and thus $L(1 / 2, F) L\left(1 / 2, F \times \chi_{-4}\right) \neq 0$.

Moreover, we get the following quadruple non-vanishing result:

Corollary 3. Let $q_{1}$ and $q_{2}$ be any two coprime fundamental discriminants and let $N$ be sufficiently large. Then, there exists $F \in S_{k}^{(2)}(N)^{\text {new, } T}$ such that

$$
L(1 / 2, F) L\left(1 / 2, F \times \chi_{-4}\right) L\left(1 / 2, F \times \chi_{q_{1}}\right) L\left(1 / 2, F \times \chi_{q_{2}}\right) \neq 0 .
$$

\subsection{Representation of integers by quadratic forms}

A positive integral $m \times m$ matrix $Q$ with even diagonal entries gives rise to a quadratic form $q(x)=\frac{1}{2} x^{T} Q x$. It is one of the classical tasks of number theory to study which numbers $n$ are represented by $q$ or more precisely to count the number of solutions

$$
r(q, n):=\#\left\{x \in \mathbb{Z}^{m} \mid q(x)=n\right\} .
$$

The first general result is due to Tartakowsky [58] who showed in 1929 by means of the Hardy-Littlewood method for $m \geqslant 5$ that $r(q, n) \geqslant 1$ if $n$ is sufficiently large and locally represented by $q$ meaning that for all primes $p$ there exists a $p$-adic solution $x_{p} \in\left(\mathbb{Z}_{p}\right)^{m}$ of $n=q\left(x_{p}\right)$.

For $m=4$ the situation becomes more subtle. Even though $x^{2}+y^{2}+7 z^{2}+7 w^{2}$ represents every $n$ locally, it fails over the integers to represent numbers of the form $37^{2 k}$ for $k \in \mathbb{Z}$. To eliminate this case, Tartakowsky makes the stronger assumption that $n$ is primitively locally represented by $q$ which means that there is a $p$-adic solution $x_{p}$ of $q\left(x_{p}\right)=n$ for all primes such that at least one entry of $x_{p}$ is a unit in $\mathbb{Z}_{p}$. With this condition, he manages to extend his result to $m=4$.

In the work of Tartakowsky the lower bound for $n$ is not made effective and it is not clear how it depends on $Q$. Especially the latter is important for many applications. The first finding in this direction is due to Watson [62] who shows that a locally represented integer is represented by $q$ if

$$
n \gg \begin{cases}(\operatorname{det} Q)^{\frac{5}{m-4}+\frac{1}{m}} & 5 \leqslant m \leqslant 9, \\ (\operatorname{det} Q)^{1+\epsilon} & m \geqslant 10 .\end{cases}
$$


By choosing another analytical approach, Hsia and Icaza [26] obtain an effective lower bound of size $(\operatorname{det} Q)^{\frac{m-2}{m-4}+\frac{2}{m}}$ which improves the exponent of Watson if $m=5,6$. Furthermore, Browning and Dietmann [12] improve the exponent in the cases $7 \leqslant m \leqslant 9$ by applying a modern version of the Hardy-Littlewood circle method, cf. [12, Table 1].

To address the case $m=4$, Browing and Dietmann make the assumption that for all primes $p$ and $k \in \mathbb{Z}$ there exists $x \in \mathbb{Z}^{4}$ such that

$$
\frac{1}{2} x^{T} Q x \equiv n\left(\bmod p^{k}\right) \quad \text { and } \quad Q x \not \equiv 0\left(\bmod p^{\tau}\right)
$$

where $\tau=1$ if $p$ is odd and $\tau=3$ for $p=2$. If this strong local solubility condition (SLC) holds, they obtain a lower bound of size $(\operatorname{det} Q)^{2} h(Q)^{8+\epsilon}$ where $h(Q)$ is the size of the largest coefficient (in absolute values) which satisfies $(\operatorname{det} Q)^{\frac{1}{4}} \leqslant h(Q) \leqslant \operatorname{det} Q$.

A much broader array of results for $m=4$ is obtained via the theory of modular forms and theta series by Rouse [45]. Let $N$ denote the level of $Q$. Rouse shows that $r(q, n) \geqslant 1$ if $n$ is locally represented by $q$ and

- $n \gg\left(N^{\frac{3}{2}}(\operatorname{det} Q)^{\frac{5}{4}}+N^{2} \operatorname{det} Q\right) N^{\epsilon} \quad$ and $\quad(n, N)=1$,

- $n \gg\left(N^{\frac{5}{4}}(\operatorname{det} Q)^{\frac{5}{4}}+N^{3} \operatorname{det} Q\right) N^{\epsilon} \quad$ and $n$ satisfies (SLC),

- $n \gg\left(N^{\frac{5}{2}}(\operatorname{det} Q)^{\frac{9}{4}}+N^{3} \operatorname{det} Q^{2}\right) N^{\epsilon} \quad$ and $n$ primitively locally represented.

In this thesis, we refine Rouse's approach and extend it to $m \geqslant 5$. The key difference is our estimation of the Petersson inner product of the cuspidal part of the theta series. By rather elementary methods that are based on [4], we obtain a considerable sharper bound than [45, Theorem 3].

For a diagonal form $q$ we let $a$ denote the product of its two largest coefficients. For all other forms, we set $a=(\operatorname{det} Q)^{\frac{2}{m}}$.

Theorem 4. Let $q(x)=x^{T} Q x$ denote a positive, integral quadratic form in $m \geqslant 4$ variables. Then, $n$ is represented by $q$ provided that $n$ is locally represented by $q$ and that

- $(n, N)=1$ and

$$
n \gg N^{1+\frac{2}{m-2}+\epsilon}+\left(\frac{N \operatorname{det} Q}{\sqrt{a}}\right)^{\frac{1}{m-2}+\epsilon} .
$$

- $m=4, n$ satisfies (SLC) and

$$
n \gg N^{3+\epsilon}+\frac{N^{1+\epsilon}(\operatorname{det} Q)^{2}}{a} .
$$


- $m=4, n$ is primitively locally represented by $q$ and

$$
n \gg\left(N+\frac{\operatorname{det} Q}{\sqrt{a}}\right) N^{3+\epsilon} \tilde{N}
$$

where $N=\tilde{N} l^{2}$ with $\tilde{N}$ squarefree.

- $m \geqslant 5$ and

$$
n \gg\left(N^{1+\frac{2}{m-3}}+\left(\frac{\operatorname{det} Q \sqrt{N}}{\sqrt{a}}\right)^{\frac{2}{m-3}}\right) N^{\epsilon} \min \left(N,(\operatorname{det} Q)^{\frac{1-\delta}{m-4}}\right)^{\frac{4}{m-3}}
$$

for any $\delta>0$ with $(\operatorname{det} Q)^{\delta} \leqslant \prod_{p \mid \operatorname{det} Q}\left(p^{2}, \operatorname{det} Q\right)$. If $n$ satisfies (SLC), we can drop the minimum term.

Note that (SLC) is satisfied for all $n$ if $N=p_{1}^{r_{1}} \ldots p_{s}^{r_{j}}$ and $p_{j}^{m+r_{j}-4} \nmid \operatorname{det} Q$. The bounds in Theorem 4 are not only an improvement in the case of $m=4$ but also for larger $m$. For example, for $m=6$ and arbitrary $n$ we obtain the condition

$$
n \gg N^{\frac{5}{3}} \min \left(N^{\frac{4}{3}},(\operatorname{det} Q)^{\frac{2}{3}-\delta}\right)+(\operatorname{det} Q)^{\frac{4}{3}} .
$$

In general Theorem 4 is favorable in comparison to previous results in those cases where the determinant is relative large compared to the level or where (SLC) is satisfied.

Next, let us outline the proof of Theorem 4. Some of the interim findings are of independent interest and have applications in other areas. Following a standard approach due to Siegel, we approximate the theta series by an average over the different classes in the genus of $Q$. The difference is a cusp form and we write $\theta(Q, z)=\theta(\operatorname{gen} Q, z)+f(z)$. On the level of Fourier coefficients, this corresponds to

$$
r(Q, n)=r(\operatorname{gen} Q, n)+a(n) .
$$

By Siegel's Hauptsatz we have that

$$
r(\operatorname{gen} Q, n)=\frac{(2 \pi)^{\frac{m}{2}} n^{\frac{m}{2}-1}}{\Gamma\left(\frac{m}{2}\right) \sqrt{\operatorname{det} Q}} \prod_{p} \beta_{p}(n, Q)
$$

where

$$
\beta_{p}(n, Q)=\lim _{a \rightarrow \infty} p^{-a(m-1)} \#\left\{m \in\left(\mathbb{Z} / p^{a} \mathbb{Z}\right)^{m} \mid \frac{1}{2} m^{T} Q m \equiv n \bmod p^{a}\right\} .
$$

To evaluate the $p$-adic densities, we follow the work of [24] and [45]. If $n$ is (primitively) locally represented by $Q$ for all primes this yields

$$
\prod_{p} \beta_{p}(n, Q) \gg \begin{cases}N^{-\epsilon} & \text { if } n \text { satisfies SLC } \\ N^{-\frac{1}{2}-\epsilon} \tilde{N}^{-\frac{1}{2}} & \text { if } m=4, \\ N^{-\epsilon} \max \left(N^{-1},(\operatorname{det} Q)^{-\frac{1}{m-4}}\right) & \text { if } m \geqslant 5,\end{cases}
$$


where $N=\tilde{N} l^{2}$ with $\tilde{N}$ squarefree. To bound the Fourier coefficients of the cusp form $f(z)=\sum_{n \geqslant 1} a(n) e(n z)$ we apply either the Petersson formula, cf. [27, Corollary 14.24], or Deligne's proof of the Ramanujan-Petersson conjecture. This yields

$$
a(n) \ll\|f\| n^{\frac{m}{4}-\frac{1}{2}} \min \left(N^{\frac{1}{2}}, 1+n^{\frac{1}{4}}(n, N)^{\frac{1}{4}} N^{-\frac{1}{2}}\right)(n N)^{\epsilon}
$$

where the norm is induced by the Petersson inner product

$$
\langle f, g\rangle=\int_{\Gamma_{0}(N) \backslash \mathbb{H}} f(z) \overline{g(z)} y^{k-2} d x d y .
$$

By refining a proof of Blomer [4], we obtain:

Lemma 5. Let $m \geqslant 3$. Then, it holds for $f(z)=\theta(Q, z)-\theta(\operatorname{gen} Q, Z)$ that

$$
\langle f, f\rangle \ll_{m}\left(\frac{N^{\frac{m}{2}}}{\left(\operatorname{det} Q, N^{\frac{m}{2}}\right)}+\frac{N}{\sqrt{a}}\right) N^{\epsilon} .
$$

As before, a is the product of the two largest coefficients if $q$ is diagonal and $(\operatorname{det} Q)^{\frac{2}{m}}$ in general.

Bounds of the cuspidal part of the theta series are useful in different contexts, see for example [5], [21] or [47].

\subsection{Representation of binary quadratic forms by quadratic forms}

The representation of integers by quadratic forms is in fact a special instance of a more general representation problem. For two integral, positive quadratic forms in $m$ and $n$ variables given by $Q$ and $2 T$ we study

$$
r(Q, T):=\#\left\{X \in M_{m, n}(\mathbb{Z}) \mid \frac{1}{2} X^{T} Q X=T\right\} .
$$

Therefore, we consider the Siegel theta series

$$
\theta(Q, Z)=\sum_{X \in M_{2 k, n}(\mathbb{Z})} e\left(\frac{1}{2} \operatorname{tr}\left(X^{T} S X Z\right)\right)=\sum_{T \in \mathcal{S}_{n}} r(Q, T) e(\operatorname{tr}(T Z))
$$

together with $\theta$ (gen $Q, Z)$ the corresponding weighted average over the genus. These are both Siegel modular forms with respect to $\Gamma_{0}^{(2)}(N)$ and the quadratic character (2). The crux of the matter is, however, that $\theta(Q, Z)-\theta(\operatorname{gen} Q, Z)$ vanishes only at zero-dimensional cusps and in general not at higher-dimensional cusps. This complicates the computation of the error term considerably. 
The first result for $n>1$ is due to Raghavan [44] who showed for $m \geqslant 2 n+3$ and $\min T \gg(\operatorname{det} T)^{\frac{1}{n}}$ that

$$
r(Q, T)=r(\operatorname{gen} Q, T)+\mathcal{O}\left((\operatorname{det} T)^{\frac{m-n-1}{2}}(\min T)^{2 n+2-\frac{m}{4}}\right)
$$

where $\min T=\min x^{T} T x$ for $0 \neq x \in \mathbb{Z}^{m}$. The idea of proof is to express the Fourier coefficients of $g(Z)=\theta(Q, Z)-\theta($ gen $Q, Z)$ by

$$
\int_{\mathfrak{E}} g(Z) \exp (-2 \pi \operatorname{tr}(T X)) d X
$$

where $Z=X+i T^{-1}$ runs over a cube $\mathfrak{E}$ of side length 1 with one corner in $i T^{-1}$. The computation is based on a generalized Farey dissection that was introduced by Siegel in [56, Section 8-9].

The Fourier coefficients of $\theta($ gen $Q, Z)$ are evaluated by Siegel's Hauptsatz:

$$
r(\operatorname{gen} Q, T)=\frac{\pi^{\frac{n(2 m-n+1)}{4}}(\operatorname{det} T)^{\frac{m-n-1}{2}}}{\prod_{j=0}^{n} \Gamma\left(\frac{m}{2}-j\right) \operatorname{det} Q^{\frac{n}{2}}} \prod_{p} \delta_{p}(Q, T) .
$$

For $m \geqslant 2 n+3$ Kitaoka [29] showed that $\prod_{p} \delta_{p}(Q, T)$ is bounded from below by a constant depending only on $N$. This gives an asymptotic formula for $r(Q, T)$ if $\min T \gg(\operatorname{det} T)^{\frac{1}{n}}$. However, latter condition is quite restrictive, as Minkowski's reduction theory already implies that $\min T \ll(\operatorname{det} T)^{\frac{1}{n}}$.

Furthermore, Kitoka [29] refined Raghavan's method and managed for $n=2$ to get rid of the dependence on $T$ of the lower bound of $\min T$. In addition, Kitaoka [33] extended the result to $m=6$ if $n=2$ and stated an asymptotic formula for $r\left(Q, t T_{0}\right)$ if $(t, N)=1, t \rightarrow \infty$ and $t T_{0}$ is locally represented by $Q$.

For even $m>4 n+4$ another approach is to decompose $\theta(Q, Z)-\theta($ gen $Q, Z)$ into a sum of Klingen-Eisenstein series and cusp forms of weight $k=\frac{m}{2}$. The former arise as lift of cusp forms of lower degree and converge for $k \geqslant 2 n+2$. Their Fourier coefficients were treated in detail by Kitaoka [31] who proved for even $k$ and $T>0$ that they are bounded by

$$
(\operatorname{det} T)^{k-\frac{3}{2}}(\min T)^{\frac{1-k}{2}} .
$$

Naturally, the Fourier coefficients of Siegel modular forms grow more slowly and are bounded by $\mathcal{O}(\operatorname{det} T)^{\frac{k}{2}-\frac{1}{2 n}}$. By $r(\operatorname{gen} Q, n) \gg_{N}(\operatorname{det} T)^{k-\frac{3}{2}}$ this gives an asymptotic formula for $r(Q, T)$ if $\min T$ goes to infinity and $m \geqslant 4 n+4$.

Recently, Ellenberg and Venkatesh [18] achieved a breakthrough by applying ergodic methods and obtained results even for the case that $m \geqslant n+3$. These findings are conditional, among other things, on the existence of primitive local solutions and that $T$ has a sufficiently large minimum and squarefree determinant. However, at present, this approach neither gives an effective bound on the sufficiently large minimum nor an asymptotic formula for $r(Q, T)$, cf [53]. 
None of these findings give an efficient lower bound for $\min T$ and $\operatorname{det} T$. For applications, it is particularly useful to know how the implied constant depends on $Q$. The aim of this thesis is to present an answer for the case $n=2$, even $m \geqslant 12$ and squarefree level. Therefore, we follow Kitaoka's strategy of decomposing $\theta(Q, Z)-\theta($ gen $Q, Z)$ into a sum of Klingen-Eisenstein series and cusp forms. This involves the following steps:

- The principal task is to obtain uniform bounds in the level for Fourier coefficients of Klingen-Eisenstein series. Therefore, we modify Kitaoka's approach for the principal congruence subgroup [34] appropriately. This even yields an explicit formula.

- Furthermore, we compute the Petersson inner product of the cuspidal part of the Siegel theta series of degree two.

- To bound the coefficients of the genus theta series from below, we rely on the work of [63] and [24] on the evaluation of $p$-adic densities.

For simplicity we assume that Klingen-Eisenstein series and cusp form transform with respect to the trivial character. By the transformation behavior of the theta series this implies that $\operatorname{det} Q$ is a square. Furthermore, we restrict ourselves to the case of prime level through most of the work. However, similar results hold for squarefree level, cf. Theorem 74, and Section 4.4 contains a sketch of proof.

Theorem 6. Let $m \geqslant 12$ with $4 \mid m$. Consider a positive, integral $m \times m$ matrix $Q$ of odd prime level $N$ such that $\operatorname{det} Q$ is a square. A binary quadratic form $T>0$ is represented by $Q$, i.e. $T=X^{T} Q X$ is soluble for $X \in \mathbb{Z}^{m \times 2}$, if $N^{m-4} \nmid \operatorname{det} Q$ and

$$
\begin{aligned}
& \min T \gg N^{1+\frac{2}{m-3}+\epsilon}+\left(\frac{\sqrt{N} \operatorname{det} Q}{\sqrt{a}}\right)^{\frac{2}{m-3}} N^{\epsilon}, \\
& \operatorname{det} T \gg N^{2+\epsilon}\left(\frac{N^{5}(\operatorname{det} Q)^{2}}{a}\right)^{\frac{2}{m-5}} .
\end{aligned}
$$

As before, $a$ is the product of the two largest coefficients of $q$ if $q$ is diagonal and $a=(\operatorname{det} Q)^{\frac{2}{m}}$ otherwise.

Remark. If $(\operatorname{det} T, N)=1$ we may drop the requirement that $N^{m-4} \nmid \operatorname{det} Q$, see Section 4.3.4. For $(\min T, N)=1$, the exponent in the lower bound of $\min T$ improves from $\frac{2}{m-3}$ to $\frac{2}{m-2}$.

The condition concerning the minimum of $T$ coincides with our results for the one-dimensional case. Indeed, if $T=X^{T} Q X$ is soluble for $X \in M_{m, 2}(\mathbb{Z})$, then also

$$
W^{T} T W=\frac{1}{2} X^{T} Q X
$$


for any $W \in \mathrm{Gl}_{2}(\mathbb{Z})$. For $T=\left(\begin{array}{cc}t_{1} & t_{2} / 2 \\ t_{2} / 2 & t_{4}\end{array}\right)$ and $W=\left(\begin{array}{cc}w_{1} & * \\ w_{3} & *\end{array}\right)$ the upper left entry of 13 implies that

$$
w_{1}^{2} t_{1}+w_{1} w_{3} t_{2}+w_{3}^{2} t_{4}=\frac{1}{2} x^{T} Q x
$$

is soluble in $x \in \mathbb{Z}^{m}$ for any coprime choice of $w_{1}, w_{3}$. Hence, in particular min $T$ is represented by $Q$ and applying Theorem 4 gives the first line of Theorem 6 .

Lastly, we outline the proof of Theorem 6. As $N$ is prime, there are only two one-dimensional cusps. Hence, every Klingen-Eisenstein series can be written as a linear combination of

$$
E\left(f_{1}, Z\right)=\sum_{M \in P(N) \backslash \Gamma_{0}^{(2)}(N)} j_{M}(Z)^{-k} f_{1}(\pi(M Z))
$$

and

$$
E_{N}\left(f_{2}, Z\right)=N^{k} \sum_{M \in P(N) \backslash \Gamma_{0}^{(2)}(N) J_{4}} j_{M}(N Z)^{-k} f_{2}(\pi(M(N Z)))
$$

for $f_{1}, f_{2} \in S_{k}(N)$ and $P(N)=\left\{M \in \Gamma_{0}^{(2)}(N) \mid \lambda(M)=(0,0,0, *)\right\}$, where $\lambda(M)$ denotes the bottom row of a matrix. This gives a decomposition

$$
\theta(Q, Z)=\theta(\operatorname{gen} Q, Z)+E\left(f_{1}, Z\right)+E_{N}\left(f_{2}, Z\right)+G(Z)
$$

for a cusp form $S$. An application of the Siegel $\Phi$-operator yields that

$$
f_{1}(z)=\theta(Q, z)-\theta(\operatorname{gen} Q, z) \text { and } f_{2}(z)=\frac{N^{k}}{\operatorname{det} Q}\left(\theta\left(N Q^{-1}, z\right)-\theta\left(\operatorname{gen} N Q^{-1}, z\right)\right) .
$$

By finding a suitable decomposition of $P(N) \backslash \Gamma_{0}^{(2)}(N)$ and $P(N) \backslash \Gamma_{0}^{(2)}(N) J_{4}$, we determine explicit formulas for the Fourier coefficients $A(T), B(T)$ of $E\left(f_{1}, Z\right)$ and $E_{N}\left(f_{2}, Z\right)$ at $T>0$, cf. Theorem 52 and 53 . As a result, we obtain that

$$
\begin{aligned}
& A(T) \ll(\operatorname{det} T)^{k-\frac{3}{2}} N^{-k+\frac{3}{2}} \sum_{\left(w_{1}, w_{3}\right)=1} t_{w}^{1-k}\left(a\left(t_{w}\right)(N, T)^{\frac{1}{2}}+b\left(t_{w}\right)\right) \delta_{N \mid t_{w}} \\
& B(T) \ll(\operatorname{det} T)^{k-\frac{3}{2}}(\operatorname{det} Q)^{-\frac{1}{2}} \sum_{\left(w_{1}, w_{3}\right)=1} t_{w}^{1-k} a\left(t_{w}\right)
\end{aligned}
$$

where

$$
T=\left(\begin{array}{cc}
t_{1} & t_{2} / 2 \\
t_{2} / 2 & t_{4}
\end{array}\right) \in \mathscr{S}, \quad t_{w}=w_{1}^{2} t_{1}+w_{1} w_{3} t_{2}+w_{3}^{2} t_{4}
$$


and $a(n), b(n)$ are the Fourier coefficients of $f_{1}$ and $f_{2}$. Interestingly, the bound for $E_{N}\left(f_{2}, Z\right)$ reflects the one-dimensional problem. More precisely, $B(T)$ is dominated by $r$ (gen $Q, T)$ if and only if $a\left(t_{w}\right)$ is dominated by $r\left(\right.$ gen $\left.Q, t_{w}\right)$ for all $\left(w_{1}, w_{2}\right)=1$ (assuming a lower bound of similar size for the $p$-adic densities).

To treat the Fourier coefficients of the Siegel cusp form, we apply Kitaoka's equivalent of the Petersson formula, cf. [15, Theorem 1.3]. This gives

$$
S(T) \ll\|G\|\left(N^{-\frac{1}{2}}(\operatorname{det} T)^{\frac{k}{2}-\frac{1}{4}}+1\right)
$$

where $\|G\|=\sqrt{\langle G, G\rangle}$ is induced by the Petersson inner product, cf. (3). A rather tedious computation shows that

$$
\langle G, G\rangle \ll \frac{N^{2 k+1}}{a} .
$$

For the $p$-adic densities

$$
\beta_{p}(Q, T)=\lim _{s \rightarrow \infty} p^{-s(2 m-3)} \#\left\{X \in M_{m, 2}\left(\mathbb{Z} / p^{s} \mathbb{Z}\right) \mid \frac{1}{2} X^{T} Q X \equiv T\left(\bmod p^{s}\right)\right\}
$$

we apply Yang's formula, [63], for odd primes which gives $\beta_{p}(Q, T) \gg 1$ if either $p^{2 k-4} \nmid \operatorname{det} Q$ or $(\operatorname{det} T, p)=1$. To compute the 2 -adic densities, we show how to lift certain solutions modulo 8 and obtain $\beta_{2}(T, p) \gg 1$ if $2^{m-8} \nmid \operatorname{det} Q$.

Notation and conventions. For an $L$-function $L(s)=\prod_{p} F_{p}\left(p^{-s}\right)$, we set $L^{N}=$ $\prod_{p \nmid N} F_{p}\left(p^{-s}\right)$. Furthermore, we set $\ell:=k-3 / 2$. We use the usual $\epsilon$-convention and all implied constants may depend on $\epsilon$. A term is negligible, if it is of size $\mathcal{O}\left(N^{-100}\right)$. By $[\cdot, \cdot],(\cdot, \cdot)$ we refer to the least common multiple respectively the greatest common divisor of two integers. Furthermore, we let $I_{n}$ denote the identity matrix of dimension $n$. To hold notation simple, we often omit the subscript when referring to $2 \times 2$ matrices, e.g. we write $I$ instead of $I_{2}$.

Moreover, we write $M_{n, m}(R)$ for the space of $n \times m$ matrices with entries in $R$ and $\mathcal{P}_{n}(R)$ for the space of symmetric $n \times n$ matrices with entries in $R$. Furthermore, we denote all positive semi-definite half-integer $n \times n$ matrices with diagonal entries by $\mathcal{S}_{n}$ and the subset that is positive definite by $\mathscr{S}_{n}$.

\section{Moments of Spinor $L$-Functions}

The aim of the following section is to compute a fourth moment of the spinor $L$-function in the level aspect. This part was published by the author in the Quarterly Journal of Mathematics, cf. [60].

We commence by reviewing the old- and newform theory for Siegel modular forms distinguishing between generic forms and Saito-Kurokawa lifts. The adelic framework and its associated representation theory allow us to construct specific 
bases of the new- and oldspace. Then, we focus on analytic properties of the spinor $L$-function. A major difficulty - estimating Satake parameter at ramified primes - is overcome by transferring cuspidal automorphic forms of $\mathrm{GSp}_{4}$ to $\mathrm{GL}_{4}$. Next, we introduce the principal tool for our estimate - the Kitaoka-Petersson formula. To treat the off diagonal terms in this spectral summation formula, we present estimates of sums over symplectic Koosterman sums. Finally, we conclude with the proof of Theorem 1 .

A short review of the most important properties of Siegel modular forms and some basic definitions can be found in the beginning of Section 4 .

\subsection{Representations, Newform Theory and Saito-Kurokawa Lifts}

Let $N$ be a squarefree integer with prime divisors $p \equiv 3(\bmod 4)$. To define the oldspace, we introduce four endomorphisms $T_{0}(p), T_{1}(p), T_{2}(p), T_{3}(p)$ of $S_{k}^{(2)}(N)$. The operator $T_{0}(p)$ is simply the identity, while $T_{1}(p)$ is the Atkin-Lehner involution that acts on $F \in S_{k}^{(2)}\left(N p^{-1}\right) \subset S_{k}^{(2)}(N)$ by $\left(T_{1}(p) F\right)(Z)=p^{k} F(p Z)$. The third operator $T_{2}(p)$ maps $F$ with Fourier coefficients as in (4) onto

$$
T_{2}(p) F=\sum_{T \in \mathscr{S}} a_{F}(p T)(\operatorname{det} p T)^{k / 2-3 / 4} e(\operatorname{tr}(T Z)),
$$

and $T_{3}(p)=T_{1}(p) \circ T_{2}(p)$, cf. [49]. We define the oldspace $S_{k}^{(2)}(N)^{\text {old }}$ in $S_{k}^{(2)}(N)$ as the sum of the spaces

$$
T_{i}(p) S_{k}^{(2)}\left(N p^{-1}\right), \quad i=0,1,2,3, \quad p \mid N
$$

and the newspace as the orthogonal complement of $S_{k}^{(2)}(N)^{\text {old }}$ inside $S_{k}^{(2)}(N)$ with respect to $(3)$. Furthermore, the space $S_{k}^{(2)}(N)$ contains a subspace of lifts from elliptic Hecke cusp forms $f$ of weight $2 k-2$ and level $N$, which we denote by $S_{k}^{(2)}(N)^{\mathrm{SK}}$. This gives us the following orthogonal decompositions:

$$
\begin{array}{rlrl}
S_{k}^{(2)}(N) & =S_{k}^{(2)}(N)^{\mathrm{T}} & & \oplus S_{k}^{(2)}(N)^{\mathrm{SK}} \\
& =S_{k}^{(2)}(N)^{\text {new }, \mathrm{T}} \oplus S_{k}^{(2)}(N)^{\text {old }, \mathrm{T}} \oplus S_{k}^{(2)}(N)^{\text {new, SK }} \oplus S_{k}^{(2)}(N)^{\text {old }, \mathrm{SK}} .
\end{array}
$$

A principal tool in the newform theory is the correspondence between Siegel cusp forms of degree two and automorphic representations on $\operatorname{GSp}(4)$, To simplify notation, we set $G=\operatorname{GSp}(4)$. We define the local analogue of $\Gamma_{0}^{(2)}(N)$ in $\mathbb{Z}_{p}$ for $p \mid N$ by

$$
P_{1}(p)=\left\{\left(\begin{array}{ll}
A & B \\
C & D
\end{array}\right) \in \mathrm{G}\left(\mathbb{Z}_{p}\right) \mid C \equiv 0\left(\bmod p \mathbb{Z}_{p}\right)\right\}
$$

and set $K_{0}(N)=\prod_{p \nmid N} G\left(\mathbb{Z}_{p}\right) \times \prod_{p \mid N} P_{1}(p)$. By strong approximation for $G$ we may write an element $g \in G(\mathbb{A})$ as $g=g_{\mathbb{Q}} g_{\infty} k_{\mathfrak{f}}$ with $g_{\mathbb{Q}} \in G(\mathbb{Q}), g_{\infty} \in G(\mathbb{R})^{+}, k_{\mathfrak{f}} \in K_{0}(N)$. 
Let $Z$ denote the center of $G$. For $F \in S_{k}^{(2)}(N)$, we denote the adelization of $F$ to be the function on $Z(\mathbb{A}) G(\mathbb{Q}) \backslash G(\mathbb{A})$ defined by

$$
\Phi_{F}(g)=\mu\left(g_{\infty}\right)^{k} j\left(g_{\infty}, i I_{2}\right)^{-k} F\left(g_{\infty}\left(i I_{2}\right)\right),
$$

where $j(g, Z)=\operatorname{det}(C Z+D)$ for $g=\left(\begin{array}{cc}A & B \\ D\end{array}\right)$. Since $G(\mathbb{Q}) \cap G(\mathbb{R})^{+} K_{0}(N)=\Gamma_{0}(N)$, $\Phi_{F}(g)$ is well defined. By setting

$$
\left\langle\Phi_{F}, \Phi_{G}\right\rangle=\frac{1}{\operatorname{vol}(Z(\mathbb{A}) G(\mathbb{Q}) \backslash G(\mathbb{A}))} \int_{Z(\mathbb{A}) G(\mathbb{Q}) \backslash G(\mathbb{A})} \phi_{F}(g) \overline{\phi_{G}(g)} d g
$$

we get a Hilbert space $L^{2}(G(\mathbb{Q}) \backslash G(\mathbb{A})$. This space contains a closed subspace $L_{0}^{2}\left(G(\mathbb{Q}) \backslash G(\mathbb{A})\right.$, the so called cuspidal subspace, containing all $\Phi \in L^{2}(G(\mathbb{Q}) \backslash G(\mathbb{A})$ satisfying

$$
\int_{N(\mathbb{Q}) \backslash N(\mathbb{A})} \Phi(n g) d n=0
$$

for each $g \in G(\mathbb{A})$ and each unipotent radical $N$ of each proper parabolic subgroup of $G$. The map $\psi: F \rightarrow \theta_{F}$ is an injection from $S_{k}(N)$ into $L_{0}^{2}(G(\mathbb{Q}) \backslash G(\mathbb{A})$. We denote the image by $V_{k}$ and note that $S_{k}(N) \rightarrow V_{k}$ is an isometry, normalized by

$$
\frac{\langle F, F\rangle}{\operatorname{vol}\left(\operatorname{Sp}(4, \mathbb{Z}) \backslash \mathbb{H}_{2}\right)}=\left\langle\Phi_{F}, \Phi_{F}\right\rangle .
$$

Furthermore, every $\Phi \in V_{k}$ satisfies $\Phi\left(g k_{\mathfrak{f}}\right)=\Phi(g)$ for $k_{\mathfrak{f}} \in K_{0}(N)$. We let $G(\mathbb{A})$ act on $\Phi \in L_{0}^{2}(G(\mathbb{Q}) \backslash G(\mathbb{A})$ by $\Phi(g) \rightarrow \Phi(g h)$ for $h \in G(\mathbb{A})$. Under this action, the space $L_{0}^{2}(G(\mathbb{Q}) \backslash G(\mathbb{A})$ decomposes into irreducible subspaces. We denote an irreducible cuspidal representation of $G(\mathbb{A})$ to be an irreducible subspace of $L_{0}^{2}(G(\mathbb{Q}) \backslash G(\mathbb{A})$.

There exists a decomposition of $V_{k}$ into

$$
V_{k}=\bigoplus_{\pi \in \mathcal{S}} V_{k}(\pi),
$$

where $V_{k}(\pi)$ is the subspace of $V_{k}$ composed of all elements that generate $\pi$ and $\mathcal{S}$ is the set of irreducible admissible representations $\pi$ of $G(\mathbb{A})$ with $V_{k}(\pi) \neq \varnothing$. Via the inverse of the adelization map, any basis of $V_{k}$ corresponds to a basis of $S_{k}^{(2)}(N)$. Hence, there is a basis of $S_{k}^{(2)}(N)$ such that every element is associated to an irreducible representation. These forms are automatically Hecke eigenforms at all places $p \nmid N$. Furthermore, two elements are orthogonal to each other if the associated representations differ. From now on, we only consider forms that correspond to irreducible representations.

An irreducible cuspidal representation $\pi$ of $G(\mathbb{A})$ is factorizable; so there exist irreducible unitary, admissible representations $\pi_{v}$ of $G\left(\mathbb{Q}_{v}\right)$ such that

$$
\pi \simeq \otimes_{v}^{\prime} \pi_{v},
$$


where the prime denotes a restricted tensor product. Accordingly, we factorize $V_{k}(\pi)$ as

$$
V_{k}(\pi)=\otimes_{v}^{\prime} V_{k}\left(\pi_{v}\right)
$$

with $V_{k}\left(\pi_{v}\right) \subseteq \pi_{v}$. We call $\phi_{p} \in \pi_{p}$ spherical if $\phi_{p}(g k)=\phi_{p}(g)$ for $k \in G\left(\mathbb{Z}_{p}\right)$. At unramified primes $(p \nmid N)$ and for $v=\infty, V_{k}\left(\pi_{v}\right)$ is one-dimensional and contains the unique (up to multiples) spherical vector in $\pi_{p}$, respectively the unique lowestweight vector in $\pi_{\infty}$. For ramified primes $V_{k}\left(\pi_{p}\right)$ is given by $\pi_{p}^{P_{1}(p)}$, meaning that $V_{k}\left(\pi_{p}\right)$ contains all $P_{1}(p)$ fixed vectors in $\pi_{p}$.

In the following, we study the local spaces $\pi_{p}^{P_{1}(p)}$ for $p \mid N$ in order to obtain an orthogonal basis for $V_{k}(\pi)$. A principal tool for this purpose is the categorization of possible local representations $\pi_{p}$ into different types, cf. for example [49, Table 1]. The underlying idea is to determine explicit, orthogonal bases for each type with respect to a suitable inner product. Then, $\Phi_{1}=\otimes_{v}^{\prime} \phi_{1, v}, \Phi_{2}=\otimes_{v}^{\prime} \phi_{2, v} \in V_{k}(\pi)$ are orthogonal, if for one place $p$ the local vectors $\phi_{1, p}, \phi_{2, p} \in V_{k}\left(\pi_{p}\right)$ are orthogonal.

The forms that generate the same representation $\pi$ are all part of the same

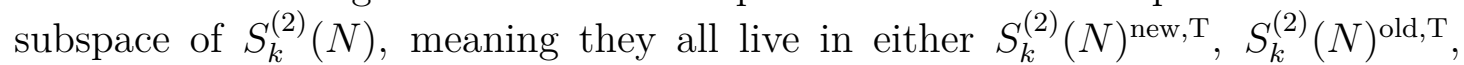
$S_{k}^{(2)}(N)^{\text {new, SK }}$ or $S_{k}^{(2)}(N)^{\text {old, SK }}$. The correspondence is determined by the local representation types of $\pi$. More precisely, a form $F \in S_{k}^{(2)}(N)$ is a newform if and only if $\pi_{F, p}$ is non-spherical (meaning that $\pi_{F, p}$ does not contain a spherical vector) at all primes $p \mid N$. If $F \in S_{k}^{(2)}(N)^{\mathrm{T}}$ then $\pi_{F, p}$ is a tempered type I representation (meaning that all characters are unitary) whenever $\pi_{F, p}$ is spherical and otherwise of type IIa, IIIa, Vb/c, VI a or VIb (conjecturally type Vb/c cannot happen). If $F \in S_{k}^{(2)}(N)^{\mathrm{SK}}$ then $\pi_{F, p}$ is type IIb whenever it is spherical, and otherwise of type VIb; cf. [17, §3].

If we take a basis of $S_{k}^{(2)}(N)$ as above and single out the forms with tempered type I for $p \nmid N$ and non-spherical $\pi_{F, p}$ for $p \mid N$, we obtain a basis of $S_{k}^{(2)}(N)^{\text {new }, T}$. By manipulating this basis locally, we can assume orthogonality and that every element is an eigenform of the $T_{2}(p)$ operator for all $p \mid N$. The latter holds if the corresponding local vectors $\phi_{p} \in \pi_{p}^{P_{1}(p)}$ are eigenforms of a certain local endomorphism $T_{1,0}$ at places $p \mid N$. (For the definition of $T_{1,0}$ see [17, p. 276].) If $\pi_{p}$ is of type IIa, $\mathrm{Vb} / \mathrm{c}, \mathrm{VI}$ there is a unique (up to multiples) $P_{1}(p)$-invariant vector $\phi_{p}$ in $\pi_{p}$ that is obviously an eigenform of $T_{1,0}$. For type IIIa, the local space has dimension two and Dickson et al, [17, §2.4], work out how to construct two orthogonal local vectors that are both eigenforms of the $T_{1,0}$ operator.

For $F \in S_{k}^{(2)}(N)^{\text {old,T }}$, there is at least one prime $p \mid N$ for which $\pi_{F, p}$ is of tempered type I, i.e. $\pi_{F, p} \simeq \chi_{1} \times \chi_{2} \rtimes \sigma$ with $\chi_{1}, \chi_{2}, \sigma$ unitary. To determine a basis for the $P_{1}(p)$ fixed subspace of $\pi_{F, p}$, we follow [49, §2]. First one determines a basis of the $I$-fixed subspace of $\pi_{F, p}$, where

$$
I=\left\{g \in G\left(\mathbb{Z}_{p}\right) \mid g \equiv\left(\begin{array}{cccc}
* & 0 & * & * \\
* & * & * & * \\
0 & 0 & * & * \\
0 & 0 & 0 & *
\end{array}\right)(\bmod p)\right\}
$$


is the Iwahori-subgroup. Let $W$ denote the 8-element Weyl group with generators $s_{1}, s_{2}$, cf. [49, §1.3], and for $w \in W$ set $f_{w}(w)=1$ and $f_{w}\left(w^{\prime}\right)=0$ for $w^{\prime} \in W, w^{\prime} \neq$ $w$. Then, a basis for the $I$-fixed subspace is given by

$$
f_{e}, f_{1}, f_{2}, f_{21}, f_{121}, f_{12}, f_{1212}, f_{212}
$$

where $f_{1}=f_{s_{1}}$ and so on. This basis is orthogonal with respect to the $G\left(\mathbb{Z}_{p}\right)$ invariant inner product

$$
\langle f, h\rangle=\int_{G\left(\mathbb{Z}_{p}\right)} f(g) \overline{h(g)} d g
$$

since the $f_{i}$ are supported on disjoint cosets. A basis for the $P_{1}(p)$ fixed subspace is then given by

$$
\phi_{1, p}=f_{e}+f_{1}, \quad \phi_{2, p}=f_{2}+f_{21}, \quad \phi_{3, p}=f_{121}+f_{12}, \quad \phi_{4, p}=f_{1212}+f_{212} .
$$

To construct an orthogonal bases of $S_{k}^{(2)}(N)^{\mathrm{T}}$, cf. [17, §3], we introduce a linear map from $S_{k}^{(2)}(e)^{\text {new,T }}$ to $S_{k}^{(2)}(a b c d e)^{\mathrm{T}}$, where $a, b, c, d, e$ denote pairwise coprime, squarefree integers. This map acts on newforms $F \in S_{k}^{(2)}(e)^{\mathrm{T}}$ in the following way: we factor the corresponding automorphic form $\phi_{F}=\phi_{S} \otimes_{p \nmid e} \phi_{p}$, where $S$ denotes the places dividing $e$. Then, for $p \mid a b c d, \phi_{p}$ is a spherical vector in a type I representation and we have an orthogonal decomposition of $\phi_{p}=\phi_{p, 1}+\phi_{p, 2}+\phi_{p, 3}+\phi_{p, 4}$ in $\pi_{p}^{P_{1}(p)}$. We set

$$
\delta_{a, b, c, d}\left(\phi_{S} \underset{p \nmid e}{\bigotimes} \phi_{p}\right)=\phi_{S} \underset{p \nmid e}{\bigotimes} \phi_{p}^{\prime}, \quad \text { where } \phi_{p}^{\prime}= \begin{cases}\phi_{p} & \text { if } p \nmid a b c d, \\ \phi_{p, 1} & \text { if } p \mid a, \\ \phi_{p, 2} & \text { if } p \mid b, \\ \phi_{p, 3} & \text { if } p \mid c, \\ \phi_{p, 4} & \text { if } p \mid d .\end{cases}
$$

Then, there is an orthogonal direct sum decomposition:

$$
S_{k}^{(2)}(N)^{\mathrm{T}}=\bigoplus_{\substack{a, b, c, d, e \\ a b c d e=N}} \delta_{a, b, c, d}\left(S_{k}^{(2)}(e)^{\mathrm{new}, \mathrm{T}}\right)
$$

For $e \neq N$, the right hand side is precisely $S_{k}^{(2)}(N)^{\text {old,T }}$.

Similarly, we construct a basis of $S_{k}^{(2)}(N)^{\mathrm{SK}}$. For this purpose, we introduce a representation theoretic analogue to the Saito-Kurokawa lifting. Consider an irreducible cuspidal automorphic representation $\pi_{0}$ of $\operatorname{PGL}(2, \mathbb{A})$ that corresponds to a form $f \in S_{2 k-2}^{(1)}(N)^{\text {new }}$. Furthermore, let $\pi_{N}$ denote the non-cuspidal automorphic representation of $\operatorname{PGL}(2, \mathbb{A})$ such that $\pi_{N, v}$ is the trivial representation if $v \nmid N$ and 
the Steinberg representation if $v \mid N$. Then, [51] introduces a functorial transfer $\Pi$ from $\mathrm{PGL}_{2} \times \mathrm{PGL}_{2}$ to $\mathrm{PGSp}_{4}$ such that $S K\left(\pi_{0}\right):=\Pi\left(\pi_{0} \times \pi_{N}\right)$ is an irreducible cuspidal automorphic representation. Furthermore, $\sigma \in V_{k}$ that generates $S K\left(\pi_{0}\right)$ is unique (up to multiples) and the corresponding cusp form coincides with the classical Saito-Kurokawa lift of $f$.

To obtain a basis of $S_{k}^{(2)}(N)^{\text {new,SK }}$ with associated representations $S K\left(\pi_{0}\right)$, we simply choose a basis of $S_{2 k-2}^{(1)}(N)^{\text {new }}$ such that the adelization of each element generates an irreducible representation $\pi_{0}$ and apply the lifting from above. If $\pi_{0, p}$ is spherical, then $\pi_{0, p}=\pi\left(\chi, \chi^{-1}\right)$ is a principal series representation of $\operatorname{PGL}\left(2, \mathbb{Q}_{p}\right)$ and by [50, §7] we have that $S K\left(\pi_{0}\right)_{p}=\chi 1_{\mathrm{GL}_{2}} \rtimes \chi^{-1}$, i.e. is of type IIb with $\sigma=\chi^{-1}$. An orthogonal basis for the $P_{1}(p)$ fixed subspace of this representation is given in [43]:

$$
\tilde{\phi}_{p, 1}=f_{e}+f_{1}, \quad \tilde{\phi}_{p, 2}=f_{2}+f_{21}+f_{121}+f_{12} \quad \tilde{\phi}_{p, 3}=f_{1212}+f_{212}
$$

with $f_{i}$ as in (16).

Let $r, s, t, m$ denote pairwise coprime, squarefree integers. Take $F \in S_{k}^{(2)}(m)^{\text {new,SK }}$ with associated representation $S K\left(\pi_{0}\right)$ and factorize $\phi_{F}=\phi_{S} \times \bigotimes_{p \nmid v}^{\prime} \phi_{p}$. For $p \mid r s t$, $\phi_{p}$ is a spherical vector in a type IIb representation as above and we have an orthogonal decomposition $\phi_{p}=\tilde{\phi}_{p, 1}+\tilde{\phi}_{p, 2}+\tilde{\phi}_{p, 3}$ in $\pi_{p}^{P_{1}(p)}$. We set

$$
\tilde{\delta}_{r, s, t}\left(\phi_{S} \bigotimes_{p \nmid e} \phi_{p}\right)=\phi_{S} \bigotimes_{p \nmid e} \phi_{p}^{\prime}, \quad \text { where } \phi_{p}^{\prime}= \begin{cases}\phi_{p} & \text { if } p \nmid r s t, \\ \tilde{\phi}_{p, 1} & \text { if } p \mid r, \\ \tilde{\phi}_{p, 2} & \text { if } p \mid s, \\ \tilde{\phi}_{p, 3} & \text { if } p \mid t .\end{cases}
$$

Then, $\tilde{\delta}_{r, s, t}$ is a mapping from $S_{k}^{(2)}(m)^{\text {new,SK }}$ to $S_{k}^{(2)}(r s t m)^{S K}$ and we have an orthogonal direct decomposition

$$
S_{k}^{(2)}(N)^{\mathrm{SK}}=\bigoplus_{\substack{r, s, t, m \\ r s t m=N}} \tilde{\delta}_{r, s, t}\left(S_{k}^{(2)}(m)^{\mathrm{new}, \mathrm{SK}}\right) .
$$

For $m \neq N$, the right hand side is $S_{k}^{(2)}(N)^{\text {old }, S K}$.

In the following section, we compute Böcherer's relation for members of these bases, 19 and (21).

\subsection{The Spinor $L$-Function and Böcherer's conjecture for oldforms}

As in the previous section, we assume that $F \in S_{k}^{(2)}(N)^{\text {new }}$ generates an irreducible representation and is an eigenform of $T_{2}(p)$ if it is not a Saito-Kurokawa lift. The former implies that $F$ is an eigenform of the Hecke algebra for all $p \nmid N$. Let 
$\alpha_{p}, \beta_{p}, \gamma_{p}$ denote the Satake parameter of $F$. Then, we define the local spin $L$ factors at $p \nmid N$ by

$$
L_{p}(s, F)=\left(1-\frac{\alpha_{p}}{p^{s}}\right)^{-1}\left(1-\frac{\alpha_{p} \beta_{p}}{p^{s}}\right)^{-1}\left(1-\frac{\alpha_{p} \gamma_{p}}{p^{s}}\right)^{-1}\left(1-\frac{\alpha_{p} \beta_{p} \gamma_{p}}{p^{s}}\right)^{-1}
$$

for $\Re s$ sufficiently large. Furthermore, we set $L_{\infty}=\Gamma_{\mathbb{C}}(s+1 / 2) \Gamma_{\mathbb{C}}(s+k-3 / 2)$, where $\Gamma_{\mathbb{C}}(s)=2(2 \pi)^{-s} \Gamma(s)$. For $F \in S_{k}^{(2)}(N)^{\text {new,T }}$, we only consider $F$ that satisfy $w_{F, N} \neq 0$. (In this case the forms $\mathrm{F}$ have a corresponding Bessel model.) This implies that for $p \mid N$, the type of $\pi_{F, N}$ is either IIIa or VIb. To distinguish these two cases, we consider the eigenvalues $\mu_{p}$ under the $T_{2}(p)$ operator. If $\mu_{p}= \pm p$, then $F$ is of type VIb. As in [51], we set for $p \mid N$

$$
\begin{array}{lll}
L_{p}(s, F)^{-1}=\left(1-\mu_{p} p^{-3 / 2-s}\right)\left(1-\mu_{p}^{-1} p^{1 / 2-s}\right) & \text { for } \mu_{p} \neq \pm p & \left(\pi_{F, p} \text { of type IIIa }\right), \\
L_{p}(s, F)^{-1}=\left(1-\mu_{p} p^{-3 / 2-s}\right)^{2} & \text { otherwise } & \left(\pi_{F, p} \text { of type VIb }\right) .
\end{array}
$$

Then, the completed $L$-function $\Lambda(s, F)=\prod_{p \cup \infty} L_{p}(s, F)$ has meromorphic continuation to the whole complex plane and satisfies the functional equation

$$
\Lambda(s, F)=N^{1-2 s} \Lambda(1-s, F) .
$$

However, this result is conditional on a nice $L$-function theory for $\mathrm{GSp}_{4}$ as in [49, 3.14]. The associated representation to $F \in S_{k}^{(2)}(N)^{\text {new, T }}$ is cuspidal and non$\mathrm{CAP}^{4}$ and thus not in one of the classes $(\mathrm{Q}),(\mathrm{P})$ and $(\mathrm{B})$ in the notion of [52]. For representations belonging to the other two possible classes, $(\mathrm{G})$ and $(\mathrm{Y})$, a nice $L$-function theory is known, cf. [52, Lemma 1.2 and 1.3].

In a standard way [27, Theorem 5.3], we get the following approximate function

$$
L\left(1 / 2, F \times \chi_{q}\right)=2 \sum_{n} \frac{A_{F}(n) \chi_{q}(n)}{n^{1 / 2}} W\left(\frac{n}{N|q|^{2}}\right)
$$

where $L(s, F)=\prod_{p} L_{p}(s, F)=\sum_{n} A_{F}(n) n^{-s}$ and

$$
W(x)=\frac{1}{2 \pi i} \int_{(2)} \frac{L_{\infty}(s+1 / 2)}{L_{\infty}(1 / 2)}\left(1-s^{2}\right) x^{-s} \frac{d s}{s} .
$$

By shifting the contour, we see immediately that the integral satisfies for all $A>0$ the bound

$$
W(x) \ll_{A}(1+x)^{-A} .
$$

\footnotetext{
${ }^{4} \mathrm{~A}$ representation of $G(\mathbb{A})$ is said to be $\mathrm{CAP}$ if it is nearly equivalent to a global induced representation of a proper parabolic subgroup of $G(\mathbb{A})$ and otherwise non-CAP. In our setting, $F$ is a Saito-Kurokawa lift if and only if the associated representation is CAP.
} 
A key role plays the following formula from Andrianov

$$
L^{N}\left(s, F \times \chi_{q}\right) a_{F}(I)=L^{N}\left(s+1 / 2, \chi_{q}\right) L^{N}\left(s+1 / 2, \chi_{-4 q}\right) \sum_{(m, N)=1} \frac{a_{F}(m I) \chi_{q}(m)}{m^{s}},
$$

cf. [1, Theorem 4.3.16] with $l=a=1, \eta=\chi=$ trivial. We denote by

$$
r(n)=r_{q}(n)=\frac{\chi_{q}(n)}{n^{1 / 2}} \sum_{d \mid n} \chi_{-4}(d)
$$

the Dirichlet coefficients of $L\left(s+1 / 2, \chi_{q}\right) L\left(s+1 / 2, \chi_{-4 q}\right)$. For $q=1$, the latter is the Dedekind zeta function $\zeta_{\mathbb{Q}(i)}(s+1 / 2)$.

For $f \in S_{2 k-2}(N)$ with $L$-function $L(s, f)$, the partial $L$-function of the corresponding Saito-Kurokawa lift $F$ is given by, cf. [41],

$$
L^{N}(s, F)=\zeta^{N}(s-1 / 2) \zeta^{N}(s+1 / 2) L^{N}(s, f) .
$$

At primes $p \mid N$, we define the local spin $L$-factors for $F \in S_{k}^{(2)}(N)^{\text {new,SK }}$ as in [11].

Let $F \in S_{k}^{(2)}(N)$ be an eigenform of the Hecke algebra at all $p \nmid N$ with eigenvalues $\lambda_{p} p^{k-3 / 2}$. Then, the eigenvalues satisfy $\lambda_{p}=p^{\frac{1}{2}}$ if $F$ is a Saito-Kurokawa lift, and due to Weissauer $\lambda_{p} \ll p^{\epsilon}$ otherwise. Furthermore, it holds by [61, Theorem 1.1] that $a_{F}(m p I)=\lambda_{p} a(m I)+|a(m I)| \mathcal{O}\left(p^{-1 / 2}\right)$, where the Fourier coefficients $a_{F}(m I)$ are normalized as in (4). It follows for $(m, N)=1$ that

$$
\begin{array}{llll}
a_{F}(m I) \ll m^{\epsilon} a_{F}(I) & \text { for } & F \in S_{k}^{(2)}(N)^{\mathrm{T}}, \\
a_{F}(m I) \ll m^{1 / 2} a_{F}(I) & \text { for } & F \in S_{k}^{(2)}(N)^{\mathrm{SK}} .
\end{array}
$$

For the proof of Theorem 1, we need to bound the coefficients $A_{F}(p)$ of the spinor $L$-function at ramified primes $p::^{5}$

Lemma 7. Let $F \in S_{k}^{(2)}(N)^{\text {new,T }}$ with $a_{F}(I) \neq 0$. Then, it holds for $p \mid N$ that

$$
\left|A_{F}(p)\right| \ll p^{-1 / 11} \text {. }
$$

Proof. Since $a_{F}(I) \neq 0$, we know that $\pi_{F, p}$ is of type IIIa or VIb. For latter we directly see $\left|A_{F}(p)\right| \ll p^{-\frac{1}{2}}$. For type IIIa, $\pi_{F, p}=\chi \rtimes \sigma \operatorname{St}_{\mathrm{GSp}(2)}$, where $\chi, \sigma$ are unramified characters of $\mathbb{Q}_{p}^{\times}$with $\chi \sigma^{2}=1$ and $\operatorname{St}_{\mathrm{GSp}(2)}$ is the Steinberg representation. Let $\omega \in \mathbb{Z}_{p}$ be a generator of the maximal ideal $p \mathbb{Z}_{p}$. By [49, Table 2], it holds that

$$
L_{p}(s, F)^{-1}=\left(1-\sigma(\omega) p^{-1 / 2-s}\right)\left(1-\sigma \chi(\omega) p^{-1 / 2-s}\right) .
$$

\footnotetext{
${ }^{5}$ The argument presented in this lemma was communicated to the author by Ralf Schmidt.
} 
The key to bound $\sigma(\omega), \sigma \chi(\omega)$ is to transfer $\pi_{F}$ to a cuspidal automorphic representation of $\mathrm{GL}(4, \mathbb{A})$. For representations in the general class $(\mathrm{G})$ in the notion of [52] such a lift is possible. Since $F$ is a non-CAP form, $\pi_{F}$ cannot be in one of the classes (Q), (P) and (B). Furthermore, [46, Table 16] states all possible representation types for $(\mathrm{Y})$ and IIIa is not one of them, so $\pi_{F}$ is in $(\mathrm{G})$.

For $\pi_{F, p}=\chi \rtimes \sigma \operatorname{St}_{\mathrm{GSp}(2)}$, the attached Langlands $L$-parameter is $(\rho, N)$ with

$$
\begin{aligned}
\rho: W_{\mathbb{Q}_{p}} & \rightarrow \operatorname{GSp}_{4}(\mathbb{C}) \\
\omega & \mapsto\left(\begin{array}{cccc}
\nu^{1 / 2} \chi \sigma(\omega) & & & \\
& \nu^{-1 / 2} \chi \sigma(\omega) & & \\
& & \nu^{1 / 2} \sigma(\omega) & \\
& & & \nu^{-1 / 2} \sigma(\omega)
\end{array}\right) \text { and } N=\left(\begin{array}{ccc}
0 & 1 & \\
0 & 0 & -1 \\
& 0 & 0
\end{array}\right),
\end{aligned}
$$

cf. [49, p. 266], where $W_{\mathbb{Q}_{p}}$ is the Weil group of $\mathbb{Q}_{p}$. We map this parameter into $\mathrm{GL}_{4}(\mathbb{C})$ and apply the local Langlands correspondence for $\mathrm{GL}_{4}$. In this way, we obtain the representation $\chi \sigma \mathrm{St}_{\mathrm{GL}(2)} \times \sigma \mathrm{St}_{\mathrm{GL}(2)}$ of $\mathrm{GL}_{4}$. Since $\chi, \sigma$ are unramified characters, this corresponds in the notation of $[8$ to the representation induced from $\mathrm{St}_{\mathrm{GL}(2)}[e(\chi \sigma)] \otimes \mathrm{St}_{\mathrm{GL}(2)}[e(\sigma)]$, where $e$ denotes the exponent of a character defined by $|\sigma|=|.|^{e(\sigma)}$. By applying [8, Theorem 1], we obtain $e(\sigma), e(\sigma \chi) \leqslant 9 / 22$. In other words, $|\sigma(\omega)|,|\sigma \chi(\omega)| \leqslant p^{9 / 22}$.

The proof of Böcherer's conjecture in [17] and [20] is obtained via local computations. In the introduction, we already stated relations for newforms; now we present similar results for members of the oldspace basis constructed in the previous section. Recall that if $F \in S_{k}^{(2)}(N)$ is an oldform, there is at least one $p \mid N$ for which $\pi_{F, p}$ is of type I or IIb. For these types, we define the local standard $L$-factor by

$$
L\left(s, \pi_{p}, \operatorname{Std}\right)^{-1}=\left(1-p^{-s}\right)\left(1-\alpha_{p} p^{-s}\right)\left(1-\alpha_{p}^{-1} p^{-s}\right)\left(1-\beta_{p} p^{-s}\right)\left(1-\beta_{p}^{-1} p^{-s}\right),
$$

where we use the following notation for the Satake parameter $\alpha_{p}, \beta_{p}, \gamma_{p}$ : If $\pi_{p}=$ $\chi_{1} \times \chi_{2} \rtimes \sigma$ is a type I representation, we set $\alpha_{p}=\chi_{1}(\omega), \beta_{p}=\chi_{2}(\omega)$ and $\gamma_{p}=\sigma(\omega)$, while for $\pi_{p}=\chi 1_{\mathrm{GL}(2)} \rtimes \sigma$, we set $\alpha_{p}=p^{-1 / 2} \chi(\omega), \beta_{p}=p^{1 / 2} \chi(\omega)$ and $\gamma_{p}=\sigma(\omega)$. In both cases, it holds that $\alpha_{p} \beta_{p} \gamma_{p}^{2}=1$. For elements in the basis (19), a proof of Böcherer's conjecture has been obtained in [17, Theorem 3.9]:

Lemma 8. Let $F \in S_{k}^{(2)}(N)^{\mathrm{T}}$. Assume that $F=\delta_{a, b, c, d}(G)$, where abcde $=N$ and $G$ is a newform in $S_{k}^{(2)}(e)^{\mathrm{T}}$. Let $\pi=\otimes_{v}^{\prime} \pi_{v}$ denote the representation attached to $G$ (or equivalently to $F$ ). Then

$$
w_{F, N}=\frac{2^{s} \pi^{5}\left(1-N^{-4}\right) \Gamma(2 k-4) L(1 / 2, F) L\left(1 / 2, F \times \chi_{-4}\right)}{N^{3} \Gamma(2 k-1) L\left(1, \pi_{F}, \mathrm{Ad}\right)} \prod_{p \mid N} \frac{J_{p}}{\left(1+p^{-1}\right)\left(1+p^{-2}\right)},
$$


where $s=6$ if $F$ is a weak Yoshida lift and 7 otherwise and

$$
J_{p}= \begin{cases}L\left(1, \pi_{p}, \operatorname{Std}\right)\left(1-p^{-4}\right) & \text { if } p \mid \text { bc }(\text { cannot occur if } F \text { newform }), \\ L\left(1, \pi_{p}, \operatorname{Std}\right)\left(1-p^{-4}\right) p^{-1} & \text { if } p \mid \text { ad }(\text { cannot occur if } F \text { newform }), \\ \left(1+p^{-2}\right)\left(1+p^{-1}\right) & \text { if } p \mid e \text { and } \pi_{p} \text { is of type IIIa, } \\ 2\left(1+p^{-2}\right)\left(1+p^{-1}\right) & \text { if } p \mid e \text { and } \pi_{p} \text { is of type VIb, } \\ 0 & \text { otherwise. }\end{cases}
$$

Analogously, we compute a relation for our basis (21) of Saito-Kurokawa lifts :

Lemma 9. Let $g \in S_{2 k-2}(m)$ denote a newform of squarefree level $m$ that generates an irreducible representation $\pi_{0}$. Let $\pi:=S K\left(\pi_{0}\right)$ denote the lift of $\pi_{0}$ and $G$ the corresponding Siegel newform. Let $r, s, t$ denote squarefree, mutually coprime numbers with $(r s t, m)=1$ and consider $F=\tilde{\delta}_{r, s, t}(G) \in S_{k}^{(2)}(N)^{\mathrm{SK}}$ where $N=$ rstm. Then:

$$
w_{F, N}=\frac{3 \cdot 2^{6} \pi^{7} \Gamma(2 k-4)}{N^{3} \Gamma(2 k-1)} \frac{L\left(1 / 2, f \times \chi_{-4}\right)}{L(3 / 2, f) L\left(1, \pi_{0}, \mathrm{Ad}\right)} \prod_{p \mid N} \frac{J_{p}}{\left(1+p^{-1}\right)\left(1+p^{-2}\right)},
$$

where

$$
J_{p}= \begin{cases}L\left(1, \pi_{p}, \operatorname{Std}\right)\left(1-p^{-4}\right) p^{-1} & \text { if } p \mid r(\text { cannot occur if } F \text { newform }), \\ L\left(1, \pi_{p}, \operatorname{Std}\right)\left(1-p^{-4}\right)\left(p^{-1}+1\right) & \text { if } p \mid s(\text { cannot occur if } F \text { newform }), \\ L\left(1, \pi_{p}, \operatorname{Std}\right)\left(1-p^{-4}\right) & \text { if } p \mid t(\text { cannot occur if } F \text { newform }), \\ 2\left(1+p^{-2}\right)\left(1+p^{-1}\right) & \text { if } p \mid m .\end{cases}
$$

Proof. By [17, (105) and (106)] we have that

$$
\frac{\left|a_{F}(I)\right|\left[\mathrm{Sp}_{4}(\mathbb{Z}): \Gamma_{0}(N)\right]}{\langle F, F\rangle}=3 \cdot 2^{1+4 k} \frac{L\left(1 / 2, \pi_{0} \times \chi_{-4}\right) L\left(1, \chi_{-4}\right)^{2}}{L\left(3 / 2, \pi_{0}\right) L\left(1, \pi_{0}, \mathrm{Ad}\right)} \sum_{p \mid N}\left(2^{-1} J^{*}\left(\phi_{p}\right)\right),
$$

where $\phi_{p} \in \pi_{p}$ is a $P_{1}(p)$ fixed vector. If $\phi_{p}$ is not spherical, then it is of type VIb and we apply the results from [17]. If $\phi_{p}$ is spherical, then by construction of $\tilde{\delta}_{r, s, t}$ it is one the three vectors from (20). We have

$$
\left.J^{*}\left(\phi_{p}\right)\right)=M(\pi) J_{0}\left(\phi_{p}\right)
$$

where

$$
\begin{aligned}
J_{0}\left(\phi_{p}\right) & =1-(q+1) q^{-3} \lambda(\phi)+q^{-2} \mu(\phi), \\
M(\pi) & =\frac{L\left(1, \pi_{F}, \operatorname{Ad}\right)\left(1+p^{-1}\right)}{\left(1-p^{-2}\right)\left(1-p^{-4}\right) L(1 / 2, \pi) L\left(1 / 2, \pi \times \chi_{-4}\right)},
\end{aligned}
$$


cf. [17, §2.5], and $\lambda(\phi), \mu(\phi)$ are given with respect to (17) by

$$
\lambda(\phi)=\frac{\left\langle d_{1} e_{1} e_{0} e_{1} \phi, \phi\right\rangle}{\langle\phi, \phi\rangle}, \quad \mu(\phi)=\frac{\left\langle d_{1} e_{0} e_{1} e_{0} \phi, \phi\right\rangle}{\langle\phi, \phi\rangle} .
$$

The action of the local operators $e_{0}, e_{1}$ are given as a $8 \times 8$ matrix with respect to (16) in [49, Lemma 2.1.1]. Here, $d_{1}=(q+1)^{-1}\left(e+e_{1}\right)$ is the so-called Siegelization and maps onto the space of $P_{1}$-fixed vectors. Recall that $\pi_{p}^{P_{1}(p)}$ is spanned by $\tilde{\phi}_{p, 1}, \tilde{\phi}_{p, 2}, \tilde{\phi}_{p, 3}$ and $\tilde{\phi}_{p, 1}+\tilde{\phi}_{p, 2}+\tilde{\phi}_{p, 3}$ is the $G\left(\mathbb{Z}_{p}\right)$ fixed vector. If we write $d_{1} e_{1} e_{0} e_{1} \tilde{\phi}_{p, i}=\sum_{j=1} c_{i j} \tilde{\phi}_{p, j}$ and $d_{1} e_{0} e_{1} e_{0} \tilde{\phi}_{p, i}=\sum_{j=1} \tilde{c}_{i j} \tilde{\phi}_{p, j}$, then $\lambda\left(\tilde{\phi}_{p, i}\right)=c_{i i}$ and $\mu\left(\tilde{\phi}_{p, i}\right)=\tilde{c}_{i i}$. A straightforward but lengthy calculation shows

$$
\begin{array}{lll}
\lambda\left(\tilde{\phi}_{p, 1}\right)=(p-1) p^{2}, & \lambda\left(\tilde{\phi}_{p, 2}\right)=\frac{p-1}{p+1} p^{2}, & \lambda\left(\tilde{\phi}_{p, 3}\right)=0, \\
\mu\left(\tilde{\phi}_{p, 1}\right)=(p-1) p^{2}, & \mu\left(\tilde{\phi}_{p, 2}\right)=p^{2}, & \mu\left(\tilde{\phi}_{p, 3}\right)=0 .
\end{array}
$$

As a consequence, we get

$$
J_{0}\left(\tilde{\phi}_{p, 1}\right)=p^{-1}, \quad J_{0}\left(\tilde{\phi}_{2}\right)=1+p^{-1}, \quad J_{0}\left(\tilde{\phi}_{3}\right)=1 .
$$

It remains to compute $M(\pi)$. The local $L$-factors are computed in [2, §3.1.2] and [49, Table 2] and it holds for $\pi$ of type IIb that

$$
\frac{L(1, \pi, \mathrm{Ad})}{L(1 / 2, \pi) L\left(1 / 2, \pi \times \chi_{-4}\right)}=\left(1-p^{-1}\right) L(1, \pi, \mathrm{Std}) .
$$

\subsection{Kitaoka-Petersson formula}

The primary tool in the proof of Theorem 1 is a spectral summation of Petersson type for Siegel cusp forms. For the full modular group, it was proved in [30] and later extended in [15] to include congruence subgroups. We quote their results and introduce some notation. Let $\Lambda$ denote all symmetric, integral 2-by-2 matrices. A major role plays a generalized Kloosterman sum

$$
K(Q, T ; C)=\sum_{D} e\left(\operatorname{tr}\left(A C^{-1} Q+C^{-1} D T\right)\right)
$$

where $Q, T \in \mathscr{S}, C \in \operatorname{Mat}_{2}(\mathbb{Z})$, the sum runs over matrices

$$
\left\{D \in \mathrm{M}_{2}(\mathbb{Z}) \bmod C \Lambda \mid\left(\begin{array}{cc}
* & * \\
C & D
\end{array}\right) \in \operatorname{Sp}_{4}(\mathbb{Z})\right\}
$$


and $A$ is any matrix such that $\left(\begin{array}{ll}A & * \\ C & D\end{array}\right) \in \operatorname{Sp}_{4}(\mathbb{Z})$. The cardinality of 31 depends only on the elementary divisors of $C$, since

$$
K\left(Q, T ; U^{-1} C V^{-1}\right)=K\left(U Q U^{T}, V^{T} T V ; C\right) \text { for } U, V \in \mathrm{GL}_{2}(\mathbb{Z}),
$$

and for $C=U^{-1}\left(\begin{array}{ll}c_{1} & \\ & c_{2}\end{array}\right) V^{-1}$ one has

$$
|K(Q, T ; C)| \leqslant c_{1}^{2} c_{2}^{1 / 2+\epsilon}\left(c_{2}, t_{4}\right)^{1 / 2},
$$

where $t_{4}$ is the $(2,2)$-entry of $T[V]$.

For a real, diagonalizable matrix $P$ with positive eigenvalues $s_{1}^{2}, s_{2}^{2}$ we write

$$
\mathcal{J}_{\ell}(P)=\int_{0}^{\pi / 2} J_{\ell}\left(4 \pi s_{1} \sin \theta\right) J_{\ell}\left(4 \pi s_{2} \sin \theta\right) \sin \theta d \theta
$$

where $J_{k}(x)$ denotes the Bessel function of weight $k$. For $J_{k}(x)$, we have the simple bounds

$$
\text { (i) } \quad J_{k}(x) \ll_{k} 1, \quad(i i) \quad J_{k}(x) \ll_{k} x^{-1 / 2} \text { and }(\text { iii }) \quad J_{k}(x) \ll_{k} x^{k}
$$

for all $x>0, k>2$. This gives us

$$
\text { (i) } \mathcal{J}_{\ell}(P) \ll|P|^{\frac{l}{2}} \quad \text { and } \quad(\text { ii }) \quad \mathcal{J}_{\ell}(P) \ll(\operatorname{det} P)^{\frac{l}{2}}(\operatorname{tr}(P))^{-\frac{l}{2}-\frac{1}{4}} .
$$

The former follows from applying (35) (iii) to both Bessel factors in (34). The latter follows by applying (35) (iii) to the Bessel factor with smaller eigenvalue and 35 ( $i i)$ to the other Bessel factor.

For two matrices $P=\left(\begin{array}{cc}p_{1} & p_{2} / 2 \\ p_{2} / 2 & p_{4}\end{array}\right) \in \mathscr{S}, S=\left(\begin{array}{cc}s_{1} & s_{2} / 2 \\ s_{2} / 2 & s_{4}\end{array}\right) \in \mathscr{S}$ and $c \in \mathbb{N}$ we define a "Salié" sum

$$
H^{ \pm}(P, S ; c)=\delta_{s_{4}=p_{4}} \sum_{d_{1}(\bmod c)}^{*} \sum_{d_{2}(\bmod c)} e\left(\frac{\overline{d_{1}} s_{4} d_{2}^{2} \mp \overline{d_{1}} p_{2} d_{2}+s_{2} d_{2}+\overline{d_{1}} p_{1}+d_{1} s_{1}}{c} \mp \frac{p_{2} s_{2}}{2 c s_{4}}\right) .
$$

This sum is relatively easy to handle. By applying the well-known bound for Gauss sums $\sum_{x(\bmod c)} e\left(\frac{a x^{2}+b x}{c}\right) \ll(a, c)^{1 / 2} c^{1 / 2}$ for the $d_{2}$ sum and estimating the $d_{1}$ sum trivially, we get

$$
\left|H^{ \pm}(P, S ; c)\right| \ll c^{3 / 2}\left(c, s_{4}\right)^{1 / 2} .
$$

For $Q \in \mathscr{S}$, we define a Poincaré series

$$
P_{Q}(Z)=\sum_{\gamma \in \Gamma_{\infty} \backslash \Gamma_{0}^{(2)}(N)} j(\gamma, Z)^{-k} e(\operatorname{tr}(Q \gamma Z))=\sum_{T \in \mathscr{S}} h_{Q}(T)(\operatorname{det} T)^{\frac{k}{2}-\frac{3}{4}} e(\operatorname{tr}(T Z)),
$$


where $\Gamma_{\infty}=\left\{\left(\begin{array}{r}I \\ I\end{array}\right) \mid S \in \Lambda\right\}$, Then, we have by [15, Proposition 2.1] that

$$
\left\langle F, P_{Q}\right\rangle=8 c(\operatorname{det} Q)^{-\frac{k}{2}+\frac{3}{4}} \overline{a_{F}(Q)}, \quad \text { with } c=\frac{\sqrt{\pi}}{4}(4 \pi)^{3-2 k} \Gamma(k-3 / 2) \Gamma(k-2)
$$

for $F \in S_{k}^{(2)}(N)$. By computing $\left\langle P_{T}, P_{Q}\right\rangle$ for $T, Q \in \mathscr{S}$ it follows

$$
8 c\left(\frac{\operatorname{det} T}{\operatorname{det} Q}\right)^{\frac{k}{2}-\frac{3}{4}} \sum_{F \in B_{k}^{(2)}(N)} \frac{a_{F}(T) \overline{a_{F}(Q)}}{\langle F, F\rangle}=h_{Q}(T)(\operatorname{det} T)^{\frac{k}{2}-\frac{3}{4}} .
$$

The Fourier coefficients of the Poincaré series, $h_{Q}(T)$, have been computed in [15]:

Lemma 10. It holds for $T, Q \in \mathscr{S}$ and even $k \geqslant 6$ that

$$
\begin{aligned}
& h_{Q}(T)(\operatorname{det} T)^{\frac{k}{2}-\frac{3}{4}}=\delta_{Q \sim T} \# \operatorname{Aut}(T) \\
& +\left(\frac{\operatorname{det} T}{\operatorname{det} Q}\right)^{\frac{k}{2}-\frac{3}{4}} \sum_{ \pm} \sum_{s} \sum_{\substack{c>1 \\
N \mid c}} \sum_{U, V} \frac{(-1)^{k / 2} \sqrt{2} \pi}{c^{3 / 2} s^{1 / 2}} H^{ \pm}\left(U Q U^{T}, V^{-1} T V^{-T}, c\right) J_{\ell}\left(\frac{4 \pi \sqrt{\operatorname{det}(T Q)}}{c s}\right) \\
& +8 \pi^{2}\left(\frac{\operatorname{det} T}{\operatorname{det} Q}\right)^{\frac{k}{2}-\frac{3}{4}} \sum_{\substack{\operatorname{det} C \neq 0 \\
N \mid C}} \frac{K(Q, T ; C)}{|\operatorname{det} C|^{3 / 2}} \mathcal{J}_{\ell}\left(T C^{-1} Q C^{-T}\right),
\end{aligned}
$$

where the sum over $U, V \in \mathrm{GL}_{2}(\mathbb{Z})$ in the second term on the right hand side runs over matrices

$$
U=\left(\begin{array}{cc}
* & * \\
u_{3} & u_{4}
\end{array}\right) /\{ \pm\}, \quad V=\left(\begin{array}{cc}
v_{1} & * \\
v_{3} & *
\end{array}\right), \quad\left(\begin{array}{ll}
u_{3} & u_{4}
\end{array}\right) Q\left(\begin{array}{l}
u_{3} \\
u_{4}
\end{array}\right)=\left(\begin{array}{ll}
-v_{3} & v_{1}
\end{array}\right) T\left(\begin{array}{c}
-v_{3} \\
v_{1}
\end{array}\right)=s .
$$

Here, $\operatorname{Aut}(T)=\left\{U \in \mathrm{GL}_{2}(\mathbb{Z}) \mid U^{T} T U=T\right\}$ and $Q \sim T$ means equivalence in the sense of quadratic forms. The sums are absolutely convergent for $k \geqslant 6$.

In the last term, [30] and [15] have the constant $1 / 2 \pi^{4}$ instead of $8 \pi^{2}$. As pointed out by [6, p. 7], this is incorrect. As in [30] and [15], we refer to the first term on the right side as diagonal term, the second as rank 1 and the third as rank 2 case.

From now on, we assume that $N \equiv 3(\bmod 4)$ is prime. The main obstacle to proving Theorem 1 is computing the rank 2 case. The decay of the Bessel function implies that we only need to consider matrices $C$ with small entries. For $\beta>0$, we set

$$
\mathscr{C}(\beta)=\left\{C=\left(\begin{array}{cc}
\tilde{c}_{1} & \tilde{c}_{2} \\
\tilde{c}_{3} & \tilde{c}_{4}
\end{array}\right) \mid 0 \neq \operatorname{det} C \ll N^{\frac{1+\beta}{\ell}}, \tilde{c}_{1}, \tilde{c}_{2}, \tilde{c}_{3}, \tilde{c}_{4} \ll N^{\frac{1+\beta}{\ell}}\right\}
$$

\footnotetext{
${ }^{6}$ There is a minor typo in [15. Proposition 2.1], there should be an additional factor of 2 on the right hand side. The reason is that the original proof from Klingen [38, §6] uses a different definition for $\Gamma_{\infty}$ that differs from the definition in [15] (that coincides with ours) by a factor 2 .
} 
and

$$
h\left(m_{1}, m_{2}, C\right)=\frac{K\left(m_{2} I, m_{1} I ; N C\right)}{N^{3}|\operatorname{det} C|^{3 / 2}} \mathcal{J}_{\ell}\left(\frac{m_{1} m_{2} C^{-1} C^{-T}}{N^{2}}\right) .
$$

Recall that $\ell=k-3 / 2$.

Lemma 11. For $m_{1}, m_{2} \ll N^{1+\epsilon}$ we have

$$
\sum_{\operatorname{det} C \neq 0} h\left(m_{1}, m_{2}, C\right)=\sum_{C \in \mathscr{C}(\beta)} h\left(m_{1}, m_{2}, C\right)+\mathcal{O}\left(N^{-1-\beta+\frac{5(1+\beta)}{2 \ell}+\epsilon}\right) .
$$

Proof. By using principal divisors, we can write $C \in \mathbb{Z}^{2 \times 2}$ uniquely as

$$
C=U^{-1}\left(\begin{array}{ll}
c_{1} & \\
& c_{2}
\end{array}\right) V^{-1}
$$

where $1 \leqslant c_{1} \mid c_{2}, U \in \mathrm{GL}_{2}(\mathbb{Z}), V \in \mathrm{GL}_{2}(\mathbb{Z}) / P\left(c_{2} / c_{1}\right)$, cf. [30, Lemma 1]. Here,

$$
P(n)=\left\{\left(\begin{array}{ll}
a & b \\
c & d
\end{array}\right) \in \mathrm{GL}_{2}(\mathbb{Z}) \mid b \equiv 0(\bmod N)\right\} .
$$

In this way, we can assign to a matrix $C$ a unique parameter $\left(U, c_{1}, c_{2}, V\right)$. For fixed $c_{1}, c_{2}, V$, we pick $U_{1} \in \mathrm{GL}_{2}(\mathbb{Z})$ such that

$$
A=U_{1}^{T}\left(\begin{array}{cc}
c_{1}^{-1} & \\
& c_{2}^{-1}
\end{array}\right) V^{T} V\left(\begin{array}{cc}
c_{1}^{-1} & \\
& c_{2}^{-1}
\end{array}\right) U_{1}
$$

is Minkowski-reduced. Then, the matrices $C$ with parameters $\left(c_{1}, c_{2}, V\right)$ are precisely the matrices

$$
C=U^{-1} U_{1}^{-1}\left(\begin{array}{ll}
c_{1} & \\
& c_{2}
\end{array}\right) V^{-1}
$$

where $U$ varies over $\mathrm{GL}_{2}(\mathbb{Z})$ and $C^{-T} C^{-1}=U^{T} A U=: A[U]$. Furthermore, by $[15$, Lemma 5.5] it holds that

$$
\sum_{V=\left(\begin{array}{l}
* v_{2} \\
* v_{4}
\end{array}\right) \in \mathrm{GL}_{2}(\mathbb{Z}) / P\left(c_{2} / c_{1}\right)}\left(c_{2}, m_{1}\left(v_{2}^{2}+v_{4}^{2}\right)\right)^{1 / 2} \ll c_{1}^{-1+\epsilon} c_{2}^{3 / 2+\epsilon} .
$$

By (33), (41) and using $\left(N c_{2}, t_{4}\right)^{1 / 2} \leqslant N^{1 / 2}\left(c_{2}, t_{4}\right)^{1 / 2}$ for $t_{4}=m_{1}\left(v_{2}^{2}+v_{4}^{2}\right)$, we get

$$
\begin{aligned}
\sum_{\operatorname{det} C \neq 0} h\left(m_{1}, m_{2}, C\right) & \ll N^{\epsilon} \sum_{c_{1} \mid c_{2}} c_{1}^{-1 / 2+\epsilon} c_{2}^{1 / 2+\epsilon}\left(\sum_{\substack{U \in \mathrm{GL}_{2}(Z) \\
\operatorname{tr} A[U] \leqslant 1}}\left(\frac{m_{1} m_{2}}{N^{2}}\right)^{\ell}(\operatorname{det} A)^{\ell / 2}\right. \\
& \left.+\sum_{\substack{U \in \mathrm{GL}_{2}(Z) \\
\operatorname{tr} A[U]>1}}\left(\frac{m_{1} m_{2}}{N^{2}}\right)^{\ell / 2-1 / 4} \operatorname{det} A^{\ell / 2} \operatorname{tr}(A[U])^{-\ell / 2-1 / 4}\right),
\end{aligned}
$$


where we applied (36) $(i)$ for the first, and (36) (ii) for the second sum. For these two sums, we have the following estimates from [30, Lemma 3], [40, Lemma 3.4]

$$
\begin{aligned}
\#\left\{U \in \mathrm{GL}_{2}(\mathbb{Z}) \mid \operatorname{tr} A[U] \leqslant 1\right\} & \ll(\operatorname{det} A)^{-1 / 2}, \\
\sum_{U \in \mathrm{GL}_{2}(\mathbb{Z}), \operatorname{tr}(A[U]>1} \operatorname{det} A^{1+\delta} \operatorname{tr}(A[U])^{-5 / 4-\delta} & \ll \operatorname{det} A^{1 / 2+\delta}
\end{aligned}
$$

for every $\delta>0$ and $A$ Minkowski-reduced.

First, we consider $C$ in $\mathcal{C}:=\left\{C \mid \operatorname{det}(C) \gg N^{(1+\beta) / l}\right\}$. By (42), (43), (44) (with $\delta=l / 2-1), m_{1}, m_{2} \ll N^{1+\epsilon}$ and $\operatorname{det} A=\left(c_{1} c_{2}\right)^{-2}$, we get

$$
\begin{aligned}
\sum_{C \in \mathcal{C}} h\left(m_{1}, m_{2}, C\right) \ll N^{\epsilon} \sum_{\substack{c_{1} \mid c_{2}, c_{1} c_{2} \gg N^{(1+\beta) / \ell}}} c_{1}^{-\ell+1 / 2+\epsilon} c_{2}^{-\ell+3 / 2+\epsilon} & \ll N^{\epsilon} \sum_{c \gg N^{(1+\beta) / \ell}} c^{-\ell+3 / 2+\epsilon} \\
& \ll N^{-1-\beta+\frac{5(1+\beta)}{2 \ell}+\epsilon} .
\end{aligned}
$$

Next, we treat $C$ in

$$
\mathcal{C}_{m}=\left\{C \mid N^{\frac{m}{\alpha}} \ll \operatorname{det} C \ll N^{\frac{m+1}{\alpha}} \text { and } \operatorname{tr}\left(C^{-T} C^{-1}\right) \gg N^{\frac{2+2 \beta}{l}-\frac{2 m}{\alpha}}>1\right\}
$$

with $\alpha \in \mathbb{Z}$, such that $2 l \mid \alpha$ and $\frac{m+1}{\alpha} \leqslant \frac{1+\beta}{l}$. By (42) and (44) with $\delta=\epsilon$, we get

$$
\sum_{C \in \mathcal{C}_{m}} h\left(m_{1}, m_{2}, C\right) \ll N^{-1-\beta+\frac{(2+2 \beta)}{l}+\frac{m(l-2)}{\alpha}+\epsilon} \sum_{c \gg N^{m / \alpha}} c^{-\ell+3 / 2+\epsilon} \ll N^{-1-\beta+\frac{5}{2 \ell}(1+\beta)+\epsilon} .
$$

Note that if $C \notin \bigcup_{m} \mathcal{C}_{m} \cup \mathcal{C}$, then $C \in \mathscr{C}(\beta)$. Indeed, the former implies that

$$
\tilde{c}_{1}^{2}+\tilde{c}_{2}^{2}+\tilde{c}_{3}^{2}+\tilde{c}_{4}^{2}=\operatorname{tr}\left(C^{-T} C^{-1}\right)(\operatorname{det} C)^{2} \ll N^{(2+2 \beta) / \ell+2 / \alpha} \text { for } C=\left(\begin{array}{cc}
\tilde{c}_{1} & \tilde{c}_{2} \\
\tilde{c}_{3} & \tilde{c}_{4}
\end{array}\right) \text {. }
$$

By choosing $\alpha$ sufficiently large, the claim follows.

Remark 12. There are $\mathcal{O}\left(N^{3(1+\beta) / \ell+\epsilon}\right)$ elements in $\mathscr{C}(\beta)$. If $\tilde{c}_{1} \tilde{c}_{4}=\operatorname{det} C$, then either $\tilde{c}_{2}$ or $\tilde{c}_{3}$ must be 0 . For $\tilde{c}_{1} \tilde{c}_{4} \neq \operatorname{det} C$ and fixed $\tilde{c}_{1}, \tilde{c}_{4}, \operatorname{det} C$ there are only $\tau\left(\tilde{c}_{1} \tilde{c}_{4}-\operatorname{det} C\right) \ll N^{\epsilon}$ choices for $\tilde{c}_{2}$ and $\tilde{c}_{3}$, since $\tilde{c}_{2} \tilde{c}_{3}=\tilde{c}_{1} \tilde{c}_{4}-\operatorname{det} C$.

\subsection{Symplectic Kloosterman sums}

This section focuses on the decomposition of the Kloosterman sum in the rank 2 term of Lemma 10. The idea is to decompose the modulus $N C$ into a large part of modulus $N$ and a small part of modulus $C$. After computing the $N$ part via a congruence condition in the proof of Theorem 1, we see that the rank 2 term is small unless $C$ has a certain form. This condition allows us to compute the remaining sum over Kloosterman sums. 
Kitaoka [30, Lemma 1-3] worked out how to decompose a Kloosterman sum for the case that $C$ is diagonal and in combination with (32), this is sufficient in most cases. However, our Kloosterman sum $K\left(\mu_{1} I, \mu_{2} I ; N C\right)$ with $\mu_{1}, \mu_{2}, N \in \mathbb{Z}$ has diagonal terms in the first two arguments and such an approach would imply non-diagonal terms in the first two arguments. However, in order to apply Lemma 15. we require a diagonal term in the second argument of the Kloosterman sum, hence, we need to adjust Kitaoka's proof slightly. To simplify notation, we set $\Gamma:=\mathrm{Sp}_{4}(\mathbb{Z})$

Lemma 13. Set $c:=\operatorname{det} C$ and assume $(c, N)=1$. Choose integers $s, t$ with $s N+t c=1$ and set $X=t c \cdot C^{-1}$. Let $Q, T \in \mathscr{S}$. Then

$$
K(Q, T, N C)=K\left(X Q X^{T}, T, N\right) K\left(s^{2} Q, T, C\right) .
$$

Proof. It holds that $\left(\begin{array}{ll}A & B \\ C & D\end{array}\right) \in \Gamma$ if and only if $A^{T} D-C^{T} B=I$ and $A^{T} C$ and $B^{T} D$ are symmetric. Since $A^{T} C$ symmetric implies that $A C^{-1}$ is symmetric, it follows that,

$$
\left(\begin{array}{cc}
A & B \\
N C & D
\end{array}\right) \in \Gamma \quad \Leftrightarrow \quad\left(\begin{array}{cc}
C^{T} A & C^{T} B-s A^{T} D \\
N I & X D
\end{array}\right),\left(\begin{array}{cc}
N A & N B-X^{T} A^{T} D \\
C & s D
\end{array}\right) \in \Gamma
$$

cf. [30, Proof of Lemma 1]. Consequently, we can show that the map

$$
\begin{aligned}
D \bmod N C \Lambda & \mapsto(X D \bmod N \Lambda, s D \bmod C \Lambda) \\
\left\{D \bmod N C \Lambda \mid\left(\begin{array}{cc}
* & * \\
N C & D
\end{array}\right) \in \Gamma\right\} & \rightarrow\left\{D \bmod N \Lambda \mid\left(\begin{array}{cc}
* & * \\
N I & D
\end{array}\right) \in \Gamma\right\} \times\left\{D \bmod C \Lambda \mid\left(\begin{array}{cc}
* & * \\
C & D
\end{array}\right) \in \Gamma\right\}
\end{aligned}
$$

is bijective. This works exactly as in the proof of [30, Lemma 2], since $N C=C N$.

By $C X+s N I=I$, we obtain

$$
\begin{gathered}
\operatorname{tr}\left(A(N C)^{-1} Q+(N C)^{-1} D T\right) \\
=\operatorname{tr}\left(\left(X^{T} C^{T} A+s N A\right) N^{-1} C^{-1} Q+N^{-1} C^{-1}(C X D+s N D) T\right) \\
=\operatorname{tr}\left(\left(X^{T} C^{T} A N^{-1} C^{-1} Q+s A C^{-1} Q+N^{-1} X D T+s C^{-1} D T\right)\right. \\
=\operatorname{tr}\left(X^{T} C^{T} A N^{-1}(s N I+X C) C^{-1} Q+s A(s N I+X C) C^{-1} Q\right) \\
\quad+\operatorname{tr}\left(N^{-1} X D T+s C^{-1} D T\right) \\
=\operatorname{tr}\left(C^{T} A N^{-1} X Q X^{T}+N^{-1} X D T\right)+\operatorname{tr}\left(N A C^{-1} s^{2} Q+C^{-1} s D T\right) \\
\quad+\operatorname{tr}\left(s X^{T} C^{T} A C^{-1} Q\right)+\operatorname{tr}(s A X Q) .
\end{gathered}
$$

Since $s X^{T} C^{T} A C^{-1} Q=\operatorname{stc} A C^{-1} Q=s A X Q$ is symmetric and integral, we conclude that

$$
\begin{aligned}
& \operatorname{tr}\left(A(N C)^{-1} Q+(N C)^{-1} D T\right) \\
& \quad \equiv \operatorname{tr}\left(C^{T} A N^{-1} X Q X^{T}+N^{-1} X D T\right)+\operatorname{tr}\left(N A C^{-1} s^{2} Q+C^{-1} s D T\right)(\bmod 1) .
\end{aligned}
$$


If the modulus is $p I$ for a prime $p$, a symplectic Kloosterman sum simplifies as follows:

Lemma 14. Let $p$ be a prime, $Q=\left(\begin{array}{cc}q_{1} & q_{2} / 2 \\ q_{2} / 2 & q_{4}\end{array}\right)$ and $T=\left(\begin{array}{cc}t_{1} & t_{2} / 2 \\ t_{2} / 2 & t_{4}\end{array}\right)$. Then

$$
K(Q, T ; p I)=\sum_{\substack{d_{1}, d_{2}, d_{4}(\bmod p) \\ p \nmid \delta}} e\left(\frac{\bar{\delta}\left(d_{4} q_{1}-d_{2} q_{2}+d_{1} q_{4}\right)+d_{1} t_{1}+d_{2} t_{2}+d_{4} t_{4}}{p}\right),
$$

where $\delta=d_{1} d_{4}-d_{2}^{2}$.

Proof. Set $D=\left(\begin{array}{ll}d_{1} & d_{2} \\ d_{3} & d_{4}\end{array}\right)$. Since $\left(\begin{array}{cc}* & * \\ C & D\end{array}\right) \in \Gamma$ if and only if $C^{-1} D$ is symmetric and $(C, D)$ is primitive. 7 it follows for $C=N I$ that $d_{2}=d_{3}$ and the sum runs over all $d_{1}, d_{2}, d_{4}$ modulo $N$ that satisfy $p \nmid \delta$. Let $\bar{\delta}$ be an integer such that $\delta \bar{\delta} \equiv 1 \bmod N$. Setting $A=\bar{\delta}\left(\begin{array}{cc}d_{4} & -d_{2} \\ -d_{2} & d_{1}\end{array}\right)$, it follows that $A^{T} C$ is symmetric and $B:=\left(A^{T} D-I_{2}\right) N^{-1} \in M_{2}(\mathbb{Z})$.

The next lemma counts the number of solutions of a congruence that arises when computing the Kloosterman sum of modulus $N$.

Lemma 15. Let $N \equiv 3(\bmod 4)$ be a prime and $h_{1}, h_{2}, c_{1}, c_{2}, c_{4} \in \mathbb{Z}$ such that $4 c_{1} c_{4}-c_{2}^{2} \not \equiv 0(\bmod N)$. Let $L\left(c_{1}, c_{2}, c_{4}, h_{1}, h_{2}\right)$ denote the number of solutions $d_{1}, d_{2}, d_{4}(\bmod N)$ of

$$
\begin{aligned}
h_{1} & \equiv a\left(d_{1}+d_{4}\right) & & (\bmod N) \\
\left(d_{1} d_{4}-d_{2}^{2}\right) h_{2} & \equiv b\left(d_{4} c_{1}-d_{2} c_{2}+d_{1} c_{4}\right) & & (\bmod N) \\
0 & \neq d_{1} d_{4}-d_{2}^{2} & & (\bmod N),
\end{aligned}
$$

where $a, b$ are arbitrary integers coprime to $N$. Then

$$
L\left(c_{1}, c_{2}, c_{4}, h_{1}, h_{2}\right)=\delta_{h_{1} \equiv h_{2} \equiv 0(\bmod N)} \delta_{c_{1} \equiv c_{4}, c_{2} \equiv 0(\bmod N)} N^{2}+\mathcal{O}(N) .
$$

Proof. First, we compute the left hand side for $h_{1} \equiv h_{2} \equiv 0(\bmod N)$. It follows by (45) that $d_{4} \equiv-d_{1}(\bmod N)$ and by (46) thus $d_{1}\left(c_{1}-c_{4}\right)+c_{2} d_{2} \equiv 0(\bmod N)$.

For $c_{1} \equiv c_{4}$ and $c_{2} \equiv 0(\bmod N)$, this congruence holds for arbitrary $d_{1}, d_{2}$. In addition, congruence 47$)$ requires that $d_{1}^{2} \not \equiv d_{4}^{2}(\bmod N)$. Since only $2 N$ pairs $d_{1}, d_{2}$ fulfill $d_{1}^{2} \equiv d_{4}^{2}(\bmod N)$, there are $N^{2}-2 N$ solutions for $d_{1}, d_{2}, d_{4}(\bmod N)$ satisfying all three congruences. On the other hand, for $c_{1} \not \equiv c_{4}$ or $c_{2} \not \equiv 0(\bmod N)$, there are less than $N+1$ solutions, since choosing $d_{1}$ already fixes $d_{4}$ and vice versa.

\footnotetext{
${ }^{7}(C, D)$ is primitive, if there exists $U=\left(\begin{array}{ll}* & * \\ C & D\end{array}\right) \in \mathrm{GL}_{4}(\mathbb{Z})$.
} 
Next, we show that for fixed $h_{1} \not \equiv 0$ or $h_{2} \not \equiv 0$ and arbitrary fixed $c_{1}, c_{2}, c_{4}$, we have $L\left(h_{1}, h_{2}, c_{1}, c_{2}, c_{4}\right) \leqslant N+1$. By (45) it holds that $d_{4} \equiv \bar{a} h_{1}-d_{1}(\bmod N)$. After substituting $h_{1}$ by $a h_{1}$ and $h_{2}$ by $b h_{2}$, (46) equals

$$
h_{2}\left(-d_{1}^{2}-d_{2}^{2}\right)+h_{2} h_{1} d_{1} \equiv d_{1}\left(c_{4}-c_{1}\right)-d_{2} c_{2}+c_{1} h_{1} \quad(\bmod N) .
$$

For $h_{2} \equiv 0$, this gives $0 \equiv\left(c_{4}-c_{1}\right) d_{1}-c_{2} d_{2}+c_{1} h_{1}(\bmod N)$. Since $h_{1} \not \equiv 0$ and either $c_{4}-c_{1}, c_{1}$ or $c_{2}$ is $\not \equiv 0(\bmod N)$, choosing $d_{1}$ fixes $d_{4}$ and vice versa. For $h_{2} \not \equiv 0$ we get

$$
\begin{aligned}
& \left(d_{1}-\overline{2}\left(c_{4}-c_{1}-h_{1}\right)\right)^{2}+\left(d_{2}-\overline{2 h_{2}} c_{2}\right)^{2} \\
& \left.\equiv h_{1} c_{1}+\overline{h_{2}}+d_{1}\right)+\left(\overline{2}\left(c_{4}-c_{1}-h_{1}\right)\right)^{2}+\left(\overline{2 h_{2}} c_{2}\right)^{2} \quad(\bmod N) .
\end{aligned}
$$

The congruence $x^{2}+y^{2} \equiv n(\bmod N)$ has $N+1$ solutions for $x, y(\bmod N)$ if $N \nmid n$, and one solution if $N \mid n$, namely $(0,0)$.

To treat the remaining sum over Kloosterman sums, we cite [6, Lemma 4]. Let $\varphi: \mathbb{Z}[i] \rightarrow \mathbb{N}$ denote Euler's totient function on $\mathbb{Z}[i]$.

Lemma 16. Let

$$
C \in \mathrm{GO}_{2}(\mathbb{Z})=\left\{\left(\begin{array}{cc}
x & y \\
\mp y & \pm x
\end{array}\right) \mid(x, y) \in \mathbb{Z}^{2} \neq\{(0,0)\}\right\}
$$

and $q_{1}, q_{2}$ two fundamental discriminants (possibly 1). Then

$$
\sum_{\substack{\mu_{1}\left(\bmod \left[q_{1}, \operatorname{det} C\right]\right) \\ \mu_{2}\left(\bmod \left[q_{1}, \operatorname{det} C\right]\right)}} \chi_{q_{1}}\left(\mu_{1}\right) \chi_{q_{2}}\left(\mu_{2}\right) K\left(\mu_{2} I, \mu_{1}, C\right)=\delta_{q_{1}=q_{2}=1}|\operatorname{det} C|^{2} \varphi(x+i y) .
$$

\subsection{Proof of Theorem 1}

The proof follows [6] closely. By the approximate functional equation (23) we have

$$
\begin{aligned}
& \sum_{F \in B_{k}^{(2)}(N)^{\text {new }, \mathrm{T}}} w_{F, N} L\left(1 / 2, F \times \chi_{q_{1}}\right) \overline{L\left(1 / 2, F \times \chi_{q_{2}}\right)} \\
= & \sum_{F \in B_{k}^{(2)}(N)^{\text {new }, \mathrm{T}}} w_{F, N} \sum_{n_{1}, n_{2}} \frac{A_{F}\left(n_{1}\right) \overline{A_{F}\left(n_{2}\right)} \chi_{q_{1}}\left(n_{1}\right) \chi_{q_{2}}\left(n_{2}\right)}{n_{1}^{1 / 2} n_{2}^{1 / 2}} W\left(\frac{n_{1}}{N\left|q_{1}\right|^{2}}\right) W\left(\frac{n_{2}}{N\left|q_{2}\right|^{2}}\right) .
\end{aligned}
$$

To apply Andrianov's formula (25), we need $n_{1}, n_{2}$ to be coprime to $N$. Hence, we need to estimate

$$
L_{N}\left(F, \chi_{q}\right):=\sum_{N \mid n} \frac{A_{F}(n) \chi_{q}(n)}{n^{1 / 2}} W\left(\frac{n}{N|q|^{2}}\right)
$$


By the decay (24) of the weight function, we truncate the sum at $n \ll N^{1+\epsilon}$ at a negligible error. By Lemma 7 we get

$$
L_{N}\left(F, \chi_{q}\right)=\frac{A_{F}(N)}{N^{1 / 2}} \sum_{n \ll N^{\epsilon}} \frac{A_{F}(n) \chi_{q}(N n)}{n^{1 / 2}} W\left(\frac{n}{|q|^{2}}\right)+\mathcal{O}\left(N^{-100}\right) \ll N^{-13 / 22+\epsilon} .
$$

By applying Cauchy Schwarz and making use of the further computations of this section, i.e. $\sum_{F} w_{F, N}\left|L^{N}\left(1 / 2, F \times \chi_{q}\right)\right|^{2} \ll N^{\epsilon}$, we can remove all terms on the right hand side of (49) with $N \mid n_{1} n_{2}$ at the cost of an error of $\mathcal{O}\left(N^{-13 / 22+\epsilon}\right)$.

For newforms that are Saito-Kurokawa lifts, we cannot apply (23). Fortunately, their contribution is very small on both sides of (49), so we can extend the basis to include lifts at the cost of a small error. For the left hand side, we apply (12) and the convexity bound for central $L$ values, getting an error of $\mathcal{O}\left(N^{-5 / 4+\epsilon}\right)$. By double Mellin inversion and (22) the right hand side of $(49)$ with $F$ running over an orthogonal basis of $S_{k}^{(2)}(N)^{\text {new,SK }}$ and $n_{1}, n_{2}$ coprime to $N$ equals

$$
\begin{gathered}
=\frac{-3(2 \pi)^{5} \Gamma(2 k-4)}{\Gamma(2 k-1)} \int_{(2)} \int_{(2)} L^{N}\left(s, \chi_{q_{1}}\right) L^{N}\left(s+1, \chi_{q_{1}}\right) L^{N}\left(s, \chi_{q_{2}}\right) L^{N}\left(s+1, \chi_{q_{2}}\right) \\
\times \sum_{f \in B_{2 k-2}^{(1)}(N)^{\text {new }}} \frac{L\left(1 / 2, f \times \chi_{-4}\right) L^{N}\left(1 / 2+s, f \times \chi_{q_{1}}\right) L^{N}\left(1 / 2+s, f \times \chi_{q_{2}}\right)}{L(3 / 2, f) L(1, f, \mathrm{Ad})} \\
\quad \times \frac{L_{\infty}\left(s+\frac{1}{2}\right)}{L_{\infty}(1 / 2)} \frac{L_{\infty}\left(t+\frac{1}{2}\right)}{L_{\infty}(1 / 2)}\left(1-s^{2}\right)\left(1-t^{2}\right) N^{s+t-3}\left|q_{1}\right|^{2 s}\left|q_{2}\right|^{2 t} \frac{d s d t}{s t},
\end{gathered}
$$

where $B_{2 k-2}^{(1)}(N)^{\text {new }}$ is an orthogonal basis of $S_{2 k-2}^{(1)}(N)^{\text {new }}$ We can shift the $s$ contour to $\Re s=\epsilon$, since the pole at $s=1$ (for $q_{1}=1$ ) cancels with the zero of $\left(1-s^{2}\right)$. In the same manner, we shift $t$-contour to $\Re t=\epsilon$. By the convexity bound, we conclude that (50) is bounded by $\mathcal{O}\left(N^{-5 / 4+\epsilon}\right)$.

By combing these estimations and applying (25), we obtain

$$
\begin{aligned}
\sum_{F \in B_{k}^{(2)}(N) \text { new }} w_{F, N} L\left(1 / 2, F \times \chi_{q_{1}}\right) L\left(1 / 2, F \times \chi_{q_{2}}\right) \\
=4 \sum_{\substack{n_{1}, n_{2} \\
m_{1}, m_{2}}}^{*} \frac{r_{1}\left(n_{1}\right) r_{2}\left(n_{2}\right) \chi_{1}\left(m_{1}\right) \chi_{2}\left(m_{2}\right)}{\left(n_{1} n_{2} m_{1} m_{2}\right)^{1 / 2}} W\left(\frac{n_{1} m_{1}}{\left|q_{1}\right|^{2} N}\right) W\left(\frac{n_{2} m_{2}}{\left|q_{2}\right|^{2} N}\right) \\
\times \sum_{F \in B_{k}^{(2)}(N)^{\text {new }}} c \frac{a_{F}\left(m_{1} I\right) \overline{a_{F}\left(m_{2} I\right)}}{\langle F, F\rangle}+\mathcal{O}\left(N^{-13 / 22+\epsilon}\right),
\end{aligned}
$$

where $\Sigma^{*}$ denotes that all summands are coprime to $N$ and the constant $c$ is given by (38). To apply Kitaoka's formula on the right hand side, the sum needs 
to run over an orthogonal basis of the whole space including oldforms. To show that we can include the oldspace at the cost of a small error, we truncate at $n_{1}, n_{2}, m_{1}, m_{2} \ll N^{1+\epsilon}$, use $r_{1}(n), r_{2}(n) \ll n^{-\frac{1}{2}+\epsilon}$ and $a_{F}(m I) \ll m^{1 / 2} a_{F}(I)$, cf. (28). Hence, the first term on the right hand side of (51) with $F$ running over a basis of the oldspace $B_{k}^{(2)}(N)^{\text {old }}$ is bounded by

$$
N^{2+\epsilon} \sum_{F \in B_{k}^{(2)}(N)^{\text {old }}} w_{F, N}
$$

We choose the orthogonal basis from $(19)$ and $(21)$ for $B_{k}^{(2)}(N)^{\text {old }}$. Since there are only $\mathcal{O}(1)$ oldforms, we conclude by Lemma 8 and 9 that 52 is bounded by $\mathcal{O}\left(N^{-1+\epsilon}\right)$.

Neglecting the error terms and applying (39), display (51) equals

$$
4 \sum_{\substack{n_{1}, n_{2} \\ m_{1}, m_{2}}}^{*} \frac{r\left(n_{1}\right) r\left(n_{2}\right) \chi_{q_{1}}\left(m_{1}\right) \chi_{q_{2}}\left(m_{2}\right)}{\left(n_{1} n_{2} m_{1} m_{2}\right)^{1 / 2}} W\left(\frac{n_{1} m_{1}}{\left|q_{1}\right|^{2} N}\right) W\left(\frac{n_{2} m_{2}}{\left|q_{2}\right|^{2} N}\right) \frac{1}{8} m_{2}^{k-3 / 2} h_{m_{2} I}\left(m_{1} I\right) .
$$

The Fourier coefficients $h_{m_{2} I}\left(m_{1} I\right)$ are given by Lemma 10 . We compute the three terms separately. The diagonal term is given by

$$
4 \sum_{n_{1}, n_{2}, m}^{*} \frac{r_{1}\left(n_{1}\right) r_{2}\left(n_{2}\right) \chi_{1}\left(m_{1}\right) \chi_{2}\left(m_{2}\right) W\left(\frac{n_{1} m}{\left|q_{1}\right|^{2} N}\right) W\left(\frac{n_{2} m}{\left|q_{2}\right|^{2} N}\right)}{\left(n_{1} n_{2} m^{2}\right)^{1 / 2}} .
$$

By double Mellin inversion, this equals

$$
\begin{aligned}
& \frac{4}{(2 \pi i)^{2}} \int_{(2)} \int_{(2)} L^{N}\left(s+1, \chi_{q_{1}}\right) L^{N}\left(s+1, \chi_{-4 q_{1}}\right) L^{N}\left(t+1, \chi_{q_{2}}\right) \\
& \times L^{N}\left(t+1, \chi_{-4 q_{2}}\right) L^{N}\left(s+t+1, \chi_{q_{1} q_{2}}\right) \frac{L_{\infty}(s+1 / 2)}{L_{\infty}(1 / 2)} \frac{L_{\infty}(t+1 / 2)}{L_{\infty}(1 / 2)} \\
& \times(1-s)^{2}(1-t)^{2} N^{s} N^{t}\left|q_{1}\right|^{2 s}\left|q_{2}\right|^{2 t} \frac{d s d s}{s t} .
\end{aligned}
$$

Next, we add the local spin $L$-factors at $N$. Therefore, we write

$$
L^{N}\left(s+1, \chi_{q_{1}}\right)=L\left(s+1, \chi_{q_{1}}\right)-\frac{\chi_{q_{1}}(N)}{N^{s+1}} L\left(s+1, \chi_{q_{1}}\right) .
$$

To bound the contribution from the second term, we shift the $t$ contour in (53) to $\Re=\epsilon$ obtaining

$$
\int_{(\epsilon)} \int_{(2)} \frac{\chi_{q_{1}}(N)}{N^{s+1}} L\left(s+q, \chi_{q_{1}}\right) L^{N}\left(s+1, \chi_{-4 q_{1}}\right) \cdots(1-t)^{2} N^{s} N^{t} \frac{d s d s}{s t} \ll N^{-1+\epsilon} .
$$

For the other four $L$-functions, this works analogously. 
To compute (53) (with local $L$-factors at $N$ ), we shift the $s$-contour to $\Re=-1+\epsilon$ picking up a pole at $s=0$ of order 1 or 2 . To estimate the remaining integral, we shift the $t$-contour to $\Re t=-1+\epsilon$, picking up a pole at $t=-s$ (since $\left(q_{1}, q_{2}\right)=1$ that can only happen if $q_{1}=q_{2}=1$ ) and a pole at $t=0$. The latter pole and the remaining integral are $\mathcal{O}\left(N^{-2+\epsilon}\right)$, while the residue at $t=-s$ equals

$\frac{-4}{2 \pi i} \int_{-1+\epsilon} \Gamma(s+1) \zeta_{\mathbb{Q}(i)}(s+1) \Gamma(1-s) \zeta_{\mathbb{Q}(i)}(1-s) \frac{\Gamma(k-1+s) \Gamma(k-1-s)}{\Gamma(k-1)^{2}}\left(1-s^{2}\right)^{2} \frac{d s}{s^{2}}$.

It is not necessary to simplify this term further, since it will cancel with another term from the rank 2 contribution.

To treat the pole at $s=0$, we shift the $t$-contour to $\Re=-1+\epsilon$ picking up a pole at $t=0$ of order at most 4 that comes from the terms $L\left(t+1, \chi_{q_{2}}\right), L^{\prime}\left(t+1, \chi_{q_{1} q_{2}}\right)$ and $t^{-1}$. The remaining integral is $\mathcal{O}\left(N^{-2+\epsilon}\right)$. The residue at $s=t=0$ is the main term in Theorem 1. For $q_{1}, q_{2} \in\{1,-4\}$, both poles have at least order 2 and we obtain a polynomial in $\log N$, whose leading coefficient can be read off after applying Taylor expansion. For $q_{1}, q_{2} \notin\{1,-4\}$ each pole is of order 1 and hence, the residue does not depend on $N$.

Next, we consider the rank 1 contribution. By the decay of $W$, we truncate $n_{1}, n_{2}, m_{1}, m_{2}$ at $\ll N^{1+\epsilon}$ at a negligible error. There are $s^{\epsilon}$ choices for $U, V$ and it must hold $\left[m_{1}, m_{2}\right] \mid s$. Hence, by (37) and $r_{1}(n), r_{2}(n) \ll n^{-\frac{1}{2}+\epsilon}$, the rank 1 contribution is bounded by

$$
N^{\epsilon} \sum_{m_{1}, m_{2} \ll N^{1+\epsilon}}^{*} \frac{1}{\left(m_{1} m_{2}\right)^{1 / 2}} \sum_{s, c} \frac{\left(N c,\left[m_{1} m_{2}\right] s\right)^{1 / 2}}{\left(\left[m_{1} m_{2}\right] s\right)^{1 / 2-\epsilon}}\left|J_{\ell}\left(\frac{4 \pi m_{1} m_{2}}{\left[m_{1} m_{2}\right] N c s}\right)\right| .
$$

Setting $d=\left(m_{1}, m_{2}\right)$, the previous display is bounded by

$$
\begin{aligned}
& N^{\epsilon} \sum_{m_{1}, m_{2}, d \ll N^{1+\epsilon}}^{*} \frac{\left(m_{1} m_{2}, N\right)^{\frac{1}{2}}}{m_{1} m_{2}} d^{-\frac{3}{2}} \sum_{s, c} c^{1 / 2} s^{\epsilon}\left|J_{\ell}\left(\frac{4 \pi d}{N c s}\right)\right| \\
& \ll N^{-1 / 2+\epsilon} \sum_{d \ll N^{1+\epsilon}} d^{-1}\left(\frac{d}{N}\right)^{l-1 / 2} \sum_{s, c} c^{1 / 2-l} s^{-l+\epsilon} \ll N^{-\frac{1}{2}+\epsilon},
\end{aligned}
$$

where we used (35) ( $i$ ii $)$ for the second step.

It remains to treat the rank 2 contribution

$$
\begin{aligned}
4 \pi^{2} \sum_{n_{1}, m_{1}, n_{2}, m_{2}}^{*} & \frac{r_{1}\left(n_{1}\right) r_{2}\left(n_{2}\right) W\left(\frac{n_{1} m_{1}}{\left|q_{1}\right|^{2} N}\right) W\left(\frac{n_{2} m_{2}}{\left|q_{2}\right|^{2} N}\right) \chi_{1}\left(m_{1}\right) \chi_{2}\left(m_{2}\right)}{\left(n_{1} m_{1} n_{2} m_{2}\right)^{1 / 2}} \\
& \times \sum_{\operatorname{det} C \neq 0} \frac{K\left(m_{2} I, m_{1} I ; N C\right)}{N^{3}|\operatorname{det} C|^{3 / 2}} \mathcal{J}_{\ell}\left(\frac{m_{1} m_{2} C^{-1} C^{-T}}{N^{2}}\right) .
\end{aligned}
$$


By (24) we truncate the $n_{1}, m_{1}, n_{2}, m_{2}$ sums at $n_{1} m_{1}, n_{2} m_{2} \ll N^{1+\epsilon}$ at the cost of a negligible error. This allows us to apply Lemma 11 which bounds the contribution of all $C \notin \mathscr{C}(\beta)$ by $\mathcal{O}\left(N^{-\beta+(5+5 \beta) / 2 l+\epsilon}\right)$. For notational simplicity, we set $c:=$ $|\operatorname{det} C|$. To apply Poisson summation, we complete the $n_{1}, m_{1}, n_{2}, m_{2}$ sum at a negligible error and split $m_{1}, m_{2}$ in residue classes modulo $N\left[c, q_{1}\right]$ and $N\left[c, q_{2}\right]$. This way, display (54) equals

$$
\begin{aligned}
& 4 \pi^{2} \sum_{n_{1}, n_{2}}^{*} \frac{r_{1}\left(n_{1}\right) r_{2}\left(n_{2}\right)}{\left(n_{1} n_{2}\right)^{1 / 2}} \sum_{C \in \mathscr{C}(\beta)} \sum_{\mu_{1}\left(\bmod N\left[c, q_{1}\right]\right)}^{*} \chi_{\mu_{2}\left(\bmod N\left[c, q_{1}\right]\right)}\left(\mu_{1}\right) \chi_{q_{2}}\left(\mu_{2}\right) \frac{K\left(\mu_{2} I, \mu_{1} I ; N C\right)}{N^{3} c^{3 / 2}} \\
\times & \sum_{\substack{m_{1} \equiv \mu_{1}\left(N\left[c, q_{1}\right]\right) \\
m_{2} \equiv \mu_{2}\left(N\left[c, q_{2}\right]\right)}} \frac{W\left(\frac{n_{1} m_{1}}{\left|q_{1}\right|^{2} N}\right) W\left(\frac{n_{2} m_{2}}{\left|q_{2}\right|^{2} N}\right)}{m_{1}^{1 / 2} m_{2}^{1 / 2}} \mathcal{J}\left(\frac{m_{1} m_{2} C^{-1} C^{-T}}{N^{2}}\right)+\mathcal{O}\left(N^{-\beta+\frac{5+5 \beta}{2 l}+\epsilon}\right) .
\end{aligned}
$$

Poisson summation yields for the main term

$$
\begin{aligned}
& 4 \pi^{2} \sum_{n_{1}, n_{2}}^{*} \frac{r_{1}\left(n_{1}\right) r_{2}\left(n_{2}\right)}{\left(n_{1} n_{2}\right)^{1 / 2}} \sum_{C \in \mathscr{C}(\beta)} \sum_{\mu_{1}\left(\bmod N\left[c, q_{1}\right]\right)}^{*} \chi_{q_{1}}\left(\mu_{1}\right) \chi_{q_{2}}\left(\mu_{2}\right) \frac{K\left(\mu_{2} I, \mu_{1} I ; N C\right)}{N^{5}\left[c, q_{1}\right]\left[c, q_{2}\right] c^{3 / 2}} \\
& \times \sum_{h_{1}, h_{2} \in \mathbb{Z}} \Psi_{n_{1}, n_{2}}\left(N C ; h_{1}, h_{2}\right) e\left(-\frac{\mu_{1} h_{1}}{N\left[c, q_{1}\right]}-\frac{\mu_{2} h_{2}}{N\left[c, q_{2}\right]}\right),
\end{aligned}
$$

where $\Psi_{n_{1}, n_{2}}\left(N C ; h_{1}, h_{2}\right)$ is

$$
\int_{\mathbb{R}} \int_{\mathbb{R}} \frac{W\left(\frac{n_{1} x_{1}}{\left|q_{1}\right|^{2} N}\right) W\left(\frac{n_{2} x_{2}}{\left|q_{2}\right|^{2} N}\right)}{\sqrt{x_{1} x_{2}}} \mathcal{J}_{\ell}\left(\frac{x_{1} x_{2} C^{-1} C^{-T}}{N^{2}}\right) e\left(\frac{x_{1} h_{1}}{N\left[c, q_{1}\right]}+\frac{x_{2} h_{2}}{N\left[c, q_{2}\right]}\right) d x_{1} d x_{2} .
$$

Substituting $x_{1}$ by $N x_{1}$ and $x_{2}$ by $N x_{2}$, this integral simplifies to

$$
N \int_{\mathbb{R}} \int_{\mathbb{R}} \frac{W\left(\frac{n_{1} x_{1}}{\left|q_{1}\right|^{2}}\right) W\left(\frac{n_{2} x_{2}}{\left|q_{2}\right|^{2}}\right)}{\sqrt{x_{1} x_{2}}} \mathcal{J}_{\ell}\left(x_{1} x_{2} C^{-1} C^{-T}\right) e\left(\frac{x_{1} h_{1}}{\left[c, q_{1}\right]}+\frac{x_{2} h_{2}}{\left[c, q_{2}\right]}\right) d x_{1} d x_{2},
$$

which we denote by $N \tilde{\Psi}_{n_{1}, n_{2}}\left(C ; h_{1}, h_{2}\right)$. By applying partial summation sufficiently often with respect to $x_{1}$ and $x_{2}$ (integrating the last term and differentiating the rest), we can truncate the $h_{1}, h_{2}$ sum at $h_{1}, h_{2} \ll N^{(1+\beta) / \ell+\epsilon}$ at a negligible error.

We choose $s, t \in \mathbb{Z}$ with $s N+t c=1$ and $\left(s, q_{2}\right)=1$. By Lemma 13 , the Kloosterman sum decomposes into

$$
K\left(\mu_{2} I, \mu_{1} I, N C\right)=K\left(\mu_{2} t^{2} c C^{-1} c C^{-T}, \mu_{1} I, N I\right) K\left(s^{2} \mu_{2} I, \mu_{1} I, C\right) .
$$

The first Kloosterman sum on the right hand side equals by Lemma 14

$$
\sum_{\substack{d_{1}, d_{2}, d_{4}(\bmod N) \\ N \nmid \delta}} e\left(\frac{\mu_{2} t^{2} \bar{\delta}\left(d_{4} c_{1}-d_{2} c_{2}+d_{1} c_{4}\right)+\mu_{1}\left(d_{1}+d_{4}\right)}{N}\right),
$$


where $c C^{-1} c C^{-T}=\left(\begin{array}{cc}c_{1} & c_{2} / 2 \\ c_{2} / 2 & c_{4}\end{array}\right)$ with $c_{1}, c_{2}, c_{4} \in \mathbb{Z}, \delta=d_{1} d_{4}-d_{2}^{2}$.

Next, we split $\mu_{i} \bmod N\left[c, q_{i}\right]$ into $\nu_{i} \bmod N$ and $\gamma_{i} \bmod \left[c, q_{i}\right]$, that is, we put $\mu_{i}=\nu_{i}\left[c, q_{i}\right]+\gamma_{i} N$. Then $\chi_{q_{i}}\left(\mu_{i}\right)=\chi_{q_{i}}\left(N \gamma_{i}\right)$. Consequently, display (55) equals

$$
\begin{aligned}
& 4 \pi^{2} \sum_{n_{1}, n_{2}}^{*} \frac{r_{1}\left(n_{1}\right) r_{2}\left(n_{2}\right)}{\left(n_{1} n_{2}\right)^{1 / 2}} \sum_{C \in \mathscr{C}(\beta)} \frac{1}{N^{4}\left[c, q_{1}\right]\left[c, q_{2}\right] c^{3 / 2}} \sum_{h_{1}, h_{2} \in \mathbb{Z}} \tilde{\Psi}_{n_{1}, n_{2}}\left(C ; h_{1}, h_{2}\right) \\
\times & \sum_{\substack{\gamma_{1}\left(\bmod \left[c, q_{1}\right]\right) \\
\gamma_{2}\left(\bmod \left[c, q_{2}\right]\right)}} \chi_{q_{1}}\left(N \gamma_{1}\right) \chi_{q_{2}}\left(N \gamma_{2}\right) K\left(s \gamma_{2} I, N \gamma_{1} I, C\right) e\left(-\frac{\gamma_{1} h_{1}}{\left[c, q_{1}\right]}-\frac{\gamma_{2} h_{2}}{\left[c, q_{2}\right]}\right) \\
\times & \sum_{\substack{d_{1}, d_{2}, d_{4}(\bmod N) \\
N \nmid \delta}} \sum_{\nu_{1} \neq 0(\bmod N)} e\left(\frac{\nu_{1}\left(\left[c, q_{1}\right]\left(d_{1}+d_{4}\right)-h_{1}\right)}{N}\right) \\
\times & \sum_{\nu_{2} \neq 0(\bmod N)} e\left(\frac{\nu_{2}\left(\left[c, q_{2}\right] t^{2} \bar{\delta}\left(d_{4} c_{1}-d_{2} c_{2}+d_{1} c_{4}\right)-h_{2}\right)}{N}\right) .
\end{aligned}
$$

Thus, we need to count the number of solutions of $\mathcal{L}_{1}: h_{1} \equiv\left[c, q_{1}\right]\left(d_{1}+d_{4}\right)(\bmod N)$ and $\mathcal{L}_{2}: h_{2} \delta \equiv\left[c, q_{2}\right] t^{2}\left(d_{4} c_{1}-d_{2} c_{2}+d_{1} c_{4}\right)(\bmod N)$. For fixed $c_{1}, c_{2}, c_{4}, h_{1}, h_{2}$, the last two lines of display (56) equal:

$$
\sum_{\substack{d_{1}, d_{2}, d_{4}(\bmod N) \\ N \nmid \delta}} N^{2} \#\left\{d_{i} \mid \mathcal{L}_{1} \cap \mathcal{L}_{2}\right\}-N\left(\#\left\{d_{i} \mid \mathcal{L}_{1}\right\}+\#\left\{d_{i} \mid \mathcal{L}_{2}\right\}\right)+1 .
$$

The first term is computed in Lemma 15 and is of size $N^{4}+\mathcal{O}\left(N^{3}\right)$ for $h_{1}=h_{2}=0$ and $c_{1}=c_{4}, c_{2}=0$ and is $\mathcal{O}\left(N^{3}\right)$ in all other cases. The second term is of size $\mathcal{O}\left(N^{3}\right)$ since both congruences already fix one $d_{i}$ modulo $N$.

The condition that $C^{T} C$ is a multiple of the identity for invertible $C$ is equivalent to $C \in \mathrm{GO}_{2}(\mathbb{Z})$, cf. (48) and [6, p. 1770]. By Remark 12, there are $\mathcal{O}\left(N^{5(1+\beta) / \ell+\epsilon}\right)$ choices for $h_{1}, h_{2}$ and the entries of $C$. Hence, display (56) equals

$$
\begin{gathered}
4 \pi^{2} \sum_{n_{1}, n_{2}}^{*} \frac{r_{1}\left(n_{1}\right) r_{2}\left(n_{2}\right)}{\left(n_{1} n_{2}\right)^{1 / 2}} \sum_{C \in \mathrm{GO}_{2}(\mathbb{Z})} \frac{\chi_{q_{2}}(N \bar{s})}{\left[c, q_{1}\right]\left[c, q_{2}\right] c^{3 / 2}} \sum_{\substack{\gamma_{1}\left(\bmod \left[c, q_{1}\right]\right) \\
\gamma_{2}\left(\bmod \left[c, q_{2}\right]\right)}} \chi_{q_{1}}\left(\gamma_{1}\right) \chi_{q_{2}}\left(\gamma_{2}\right) K\left(\gamma_{2} I, \gamma_{1} I, C\right) \\
\int_{\mathbb{R}} \int_{\mathbb{R}} \frac{W\left(n_{1} x_{1} /\left|q_{1}\right|^{2}\right) W\left(n_{2} x_{2}\left|q_{2}\right|^{2}\right)}{\sqrt{x_{1} x_{2}}} \mathcal{J}_{\ell}\left(x_{1} x_{2} C^{-1} C^{-T}\right) d x_{1} d x_{2}+\mathcal{O}\left(N^{-1+\frac{5+5 \beta}{\ell}+\epsilon}\right) .
\end{gathered}
$$

We choose $\beta$ such that $\mathcal{O}\left(N^{-1+\frac{5+5 \beta}{l}+\epsilon}\right)$ is of the same size as the previous error term $\mathcal{O}\left(N^{-\beta+\frac{5+5 \beta)}{2 \ell}+\epsilon}\right)$, i.e. $\beta=\frac{2 \ell-5}{2 \ell+5}=\frac{2 k-8}{2 k+2}$.

Applying Lemma 16, we see that the sum vanishes for $q_{1} \neq 1$ or $q_{2} \neq 1$. Since matrices $C \in \mathrm{GO}_{2}(\mathbb{Z})$ fulfill $C^{-1}=C^{T} c^{-1}$, the main term of the previous display 
equals

$$
8 \pi^{2} \sum_{n_{1}, n_{2}}^{*} \frac{r_{1}\left(n_{1}\right) r_{2}\left(n_{2}\right)}{\left(n_{1} n_{2}\right)^{1 / 2}} \sum_{\substack{\gamma \in \mathbb{Z}[i] \\|\gamma|^{2} \ll N^{\frac{1+\beta}{l}+\epsilon}}} \frac{\phi(\gamma)}{|\gamma|^{3}} \int_{\mathbb{R}} \int_{\mathbb{R}} \int_{0}^{\pi / 2} \frac{W\left(n_{1} x_{1}\right) W\left(n_{2} x_{2}\right)}{\sqrt{x_{1} x_{2}}} \mathcal{J}_{\ell}\left(\frac{x_{1} x_{2}}{|\gamma|^{2}} I\right) d x_{1} d x_{2} .
$$

By (24) and (35) we can complete the $\gamma$-sum at a negligible error. By the computation in [6, §7], the resulting term equals

$$
\begin{array}{r}
16 \pi^{2} \int_{(2)} \sum_{n_{1}, n_{2}} \frac{r_{1}\left(n_{1}\right) r_{2}\left(n_{2}\right)}{\left(n_{1} n_{2}\right)^{1+s / 2}} \cdot \frac{\zeta_{\mathbb{Q}(i)}\left(\frac{s+1}{2}\right)}{\zeta_{\mathbb{Q}(i)}\left(\frac{s+3}{2}\right)}\left(\frac{L_{\infty}(1+s / 2)\left(1-\frac{s+1}{2}\right)^{2}}{L_{\infty}(1 / 2)(s+1) / 2}\right)^{2} \\
\times \frac{(4 \pi)^{s} \Gamma\left(k-\frac{3+s}{2}\right)}{(1+s) \Gamma\left(k-\frac{s-1}{2}\right)} \frac{d s}{2 \pi i} .
\end{array}
$$

We add the summands $n_{1}, n_{2}$ that are multiples of $N$ at the cost of an error of size $\mathcal{O}\left(N^{-5 / 2}\right)$. By changing variables, applying the functional equation of the Dedekind zeta-function and shifting the contour, the previous term equals

$$
\frac{4}{2 \pi i} \int_{-1+\epsilon} \zeta_{\mathbb{Q}(i)}(s+1) \zeta_{\mathbb{Q}(i)}(1-s) \frac{\Gamma(k-1+s) \Gamma(k-1-s) \Gamma(s+1) \Gamma(1-s)}{\Gamma(k-1)^{2}}\left(1-s^{2}\right)^{2} \frac{d s}{s^{2}}
$$

which cancels with the residue $s=-t$ from the rank 0 case.

\section{Theta series of degree one}

We consider an integral, positive $m \times m$ matrix $Q$ with even diagonal and its corresponding quadratic form $q(x)=\frac{1}{2} x^{T} Q x$ for $m \geqslant 3$. Throughout this thesis we tacitly assume that $q(x)$ is primitive which implies that $N Q^{-1}$ is of level $N$. The aim of this section is to obtain an asymptotic formula for

$$
r(Q, n)=\#\left\{x \in \mathbb{Z}^{m} \mid q(x)=n\right\}
$$

that is uniform in the level $N$ of $Q$. Therefore, we consider the theta series

$$
\theta(Q, z)=\sum_{x \in \mathbb{Z}^{m}} e\left(\frac{1}{2} x^{T} Q x z\right)=\sum_{n \geqslant 0} r(Q, n) e(n z) .
$$

We decompose $\theta(Q, z)$ into an Eisenstein series and a cusp form. Recall that two matrices $Q, Q^{\prime}$ are in the same genus, if there exists $U_{p} \in \mathrm{GL}_{m}\left(\mathbb{Z}_{p}\right)$ for every $p$ such that $Q=U_{p}^{T} Q^{\prime} U_{p}$. For positive $Q$ there are only finitely many classes in the genus and the set of automorphs $o(Q):=\left\{U \in \mathrm{GL}_{2}(\mathbb{Z}) \mid U^{T} Q U=Q\right\}$ is finite. We set

$$
\theta(\operatorname{gen} Q, z)=\left(\sum_{R \in \operatorname{gen} Q} \frac{1}{\# o(R)}\right)^{-1} \sum_{R \in \operatorname{gen} Q} \frac{\theta(R, Z)}{\# o(R)} \text {. }
$$


The Fourier coefficients $r$ (gen $Q, n)$ of $\theta(\operatorname{gen} Q, z)$ are given by Siegel's Hauptsatz:

$$
r(\text { gen } Q, n)=\frac{(2 \pi)^{\frac{m}{2}} n^{\frac{m}{2}-1}}{\Gamma\left(\frac{m}{2}\right) \sqrt{\operatorname{det} Q}} \prod_{p} \beta_{p}(n, Q)
$$

where $\beta_{p}(n, Q)=\lim _{a \rightarrow \infty} p^{-a(m-1)} r_{p^{a}}(n, Q)$ denotes the usual $p$-adic density with

$$
r_{p^{a}}(n, Q)=\#\left\{m \in\left(\mathbb{Z} / p^{a} \mathbb{Z}\right)^{m} \mid \frac{1}{2} m^{T} Q m \equiv n \bmod p^{a}\right\}
$$

We write $\theta(Q, Z)=\theta(\operatorname{gen} Q, z)+f(z)$ for a cusp form $f$. On the level of Fourier coefficients this corresponds to

$$
r(Q, n)=r(\operatorname{gen} Q, n)+a(n) .
$$

We view $r$ (gen $Q, n)$ as the main term and bound it from below by estimating the $p$-adic densities. To evaluate the error term $a(n)$ uniformly in the level we compute an inner product of the cuspidal part of the theta series and apply the Petersson formula. In the end we give bounds with respect to $N$ on the largest integer $n$ that is locally represented but not over the integers.

We start by treating the $p$-adic densities. If $p \nmid 2 n N$, an easy computation shows, cf. [55, Hilfssatz 12], that

$$
\beta_{p}(n, Q)= \begin{cases}1-p^{-\frac{m}{2}}\left(\frac{(-1)^{\frac{m}{2} \operatorname{det} Q}}{p^{\frac{m}{2}}}\right) & \text { if } m \text { even, } \\ 1+p^{\frac{1-m}{2}}\left(\frac{(-1)^{\frac{m-1}{2}} n \operatorname{det} Q}{p^{\frac{m}{2}}}\right) & \text { if } m \text { odd }\end{cases}
$$

For $p \nmid 2 N$, we have by [55, Hilfssatz 16] that

$$
1-p^{-r} \leqslant \beta_{p}(n, Q) \leqslant 1+p^{r}
$$

where $r=\frac{m}{2}$ for even $m$ and $r=\frac{1-m}{2}$ for odd $m$. For the remaining $p$-adic densities, we apply a formula from Yang [63]. Therefore, we fix some notation. For odd $p$ we know that $Q$ is $p$-adically equivalent to

$$
\operatorname{diag}\left(p^{\nu_{1}} u_{1}, p^{\nu_{2}} u_{2}, \ldots, p^{\nu_{m}} u_{m}\right) \quad \text { with } u_{i} \in \mathbb{Z}_{p}^{*}, 0=\nu_{1} \leqslant \nu_{2} \leqslant \ldots \leqslant \nu_{m} .
$$

We set

$$
V(l)=\left\{1 \leqslant i \leqslant m \mid \nu_{i}-l<0 \text { is odd }\right\}
$$

and

$$
d(l)=l+\frac{1}{2} \sum_{\nu_{i}<l}\left(\nu_{i}-l\right), \quad v(l)=\left(\frac{-1}{p}\right)^{\left\lfloor\frac{\# V(l)}{2}\right\rfloor} \prod_{i \in V(l)}\left(\frac{u_{i}}{p}\right) .
$$

Then, the following formula for the $p$-adic densities was shown by Yang [63, Theorem 3.1]: 
Theorem 17. Set $n=p^{a} t$ with $(t, p)=1$. Then, for odd $p$, we have that

$$
\beta_{p}(Q, n)=1+\left(1+p^{-1}\right) \sum_{\substack{0<l \leqslant a \\ \# V(l) \text { even }}} v(l) p^{d(l)}+v(a+1) p^{d(a+1)} f(n)
$$

where

$$
f(n)=\left\{\begin{array}{ll}
-\frac{1}{p} & \text { if } \# V(a+1) \text { is even, } \\
\left(\frac{t}{p}\right) \frac{1}{\sqrt{p}} & \text { if } \# V(a+1) \text { is odd. }
\end{array} .\right.
$$

The case $p=2$ is slightly more complex. However, a very similar formula holds due to Yang, cf. [63, Theorem 4.1]. A direct consequence is the following estimate for odd $p \nmid n$ :

Corollary 18. Assume that $\beta_{p}(Q, n)>0$. Then, we have for odd $p \nmid n$ either that $\beta_{p}(Q, n)=2$ or

$$
1-\frac{1}{p} \leqslant \beta_{p}(Q, n) \leqslant 1+\frac{1}{p}
$$

Proof. We apply Theorem 17 with $a=0$ and make a case distinction according to $\# V(1)=\#\left\{1 \leqslant i \leqslant m \mid \nu_{i}=0\right\}$. If $\# V(1)=1$, then

$$
\beta_{p}(Q, n)=1+\left(\frac{n}{p} \prod_{i \in V(1)}\left(\frac{u_{i}}{p}\right)\right)=2
$$

since $\beta_{p}(Q, n)>0$. For $\# V(1) \geqslant 2$, we have $p^{d(1)}|f(n)| \leqslant p^{-1}$ and the formula yields $1-\frac{1}{p} \leqslant \beta_{p}(Q, n) \leqslant 1+\frac{1}{p}$.

Furthermore, we obtain the following upper bounds for the $p$-adic densities:

Corollary 19. It holds that

$$
\prod_{p} \beta_{p}(Q, n) \ll(n, N)^{\frac{1}{2}}(n N)^{\epsilon} .
$$

Proof. By (58), 59) and Corollary 18 we already know that

$$
\prod_{p} \beta_{p}(Q, n) \ll(n N)^{\epsilon} \prod_{p \mid 2(n, N)} \beta_{p}(Q, n) .
$$

Let $(n, N)=p^{b}$. Then, $d(l) \leqslant \frac{b}{2}$ for all $l$. Hence, Theorem 17 gives for odd $p$ that $\beta_{p}(Q, n) \ll p^{\frac{b}{2}}$ as $|v(l)|=1$. For $p=2$ we apply [63, Theorem 4.1]. The term $d(k)$, defined in [63, (4.3)], satisfies $d(k) \leqslant 2^{\frac{b+1}{2}}$ which implies that [63, (4.4)] is bounded by $\ll 2^{\frac{b}{2}}$ and hence, also $\beta_{2}(n, Q)$. 
As a consequence, we obtain by (57) that

$$
r(\operatorname{gen} Q, n) \ll \frac{n^{\frac{m}{2}-1}(n, N)^{\frac{1}{2}}}{\sqrt{\operatorname{det} Q}}(n N)^{\epsilon} .
$$

To bound $\beta_{p}(Q, n)$ from below, we make the assumption that $n$ is locally represented by $q$. For $m \geqslant 5$ this implies that there is a primitive local solution, i.e., a $p$-adic solution of $q(x)=n$ such that at least one of the entries of $x$ is a unit in $\mathbb{Z}_{p}$. If such a solution exists, we always have $\beta_{p}(n, Q)>0$. For four variables the situation becomes more complicated, since the quadratic form might be anisotropic over $\mathbb{Z}_{p}$ for some prime $p$ which means that $\lim _{v \rightarrow \infty} \beta_{p}\left(p^{v}, Q\right)=0$. To avoid complications we assume that $n$ is primitively represented by $q$ if $m=4$.

We follow the approach from Hanke [24]. As before, we use that $q(t)$ is $p$-adically equivalent to a form

$$
p^{\nu_{1}} g_{1}\left(t_{1}\right)+p^{\nu_{2}} g_{2}\left(t_{2}\right)+\ldots+p^{\nu_{r}} g_{r}\left(t_{r}\right),
$$

where $g_{i}$ has unit discriminant and is indecomposable over $\mathbb{Z}_{p}$ and $p^{\nu_{i}} \mid N$ for all $i$. If $p$ is odd, this implies that $g_{i}$ is of dimension 1, while for $p=2$ the forms $g_{i}$ may also be equivalent to one the binary forms $x y$ or $x^{2}+x y+y^{2}$.

We call a solution of $q(t) \equiv n\left(\bmod p^{r}\right) \operatorname{good}$ if there is $i$ such that $p^{\nu_{i}} t_{i} \neq 0(\bmod p)$ and $b a d$ if $t_{i} \equiv 0(\bmod p)$ for all $\nu_{i}=0$, but there is some $t_{j} \neq \equiv(\bmod p)$ with $\nu_{j} \geqslant 1$. For $\nu_{j}=1$ we refer to this solution as type I and for $\nu_{j} \geqslant 2$ as type II. Furthermore, we say that $t$ is of zero type if $t \equiv 0(\bmod p)$.

We denote the number of good solutions modulo $p^{a}$ by $r_{p^{a}}^{\text {good }}(n, Q)$. We infer by Hensel's Lemma for odd $p$ that

$$
r_{p^{a+1}}^{\text {good }}(n, Q)=p^{a(m-1)} r_{p}^{\text {good }}(n, Q) .
$$

and $r_{2^{a}}^{\text {good }}(n, Q)=2^{(a-2)(m-1)} r_{2^{3}}^{\text {good }}(n, Q)$.

For odd $p$ and $(n, p)=1$ we already treated the $p$-adic densities in Corollary 18 . If $p \mid n$, we decompose 62 int 8

$$
q_{0}\left(\vec{x}_{0}\right)+p q_{1}\left(\vec{x}_{1}\right)
$$

where $q_{0}$ is of discriminant coprime to $p$ and $q_{0}, q_{1}$ are diagonal forms. We make a distinction according to $\operatorname{dim} q_{0}=\#\left\{1 \leqslant i \leqslant m \mid \nu_{i}=0\right\}$. If $\operatorname{dim} q_{0}=1$, there are no good solutions. For $\operatorname{dim} q_{0}=2$, the existence of one primitive solution of $u_{1} t_{1}^{2}+u_{2} t_{2}^{2} \equiv 0(\bmod p)$ implies that there are $p-1$ non-trivial solution. By choosing all other entries, $\vec{x}_{1}, \vec{x}_{2}$, arbitrarily, there are $p^{m-1}+p^{m-2}$ good solutions of $q(\vec{x}) \equiv 0(\bmod p)$. For $\operatorname{dim} q_{0}=3$, a similar $\operatorname{argument}$ works, cf. [45, Proof of Lemma 2]. If $\operatorname{dim} q_{0} \geqslant 4$, we apply Theorem 17 and the fact that $d_{l} \leqslant-l$ gives the bound $r_{p}(n) \geqslant 1-\frac{1}{p}-\frac{2}{p^{2}}$.

\footnotetext{
${ }^{8}$ We use the notation $\vec{x}_{i}$ here to emphasize that $\vec{x}_{i}$ is a vector.
} 
For $p=2$, we consider

$$
q_{0}\left(\vec{x}_{0}\right)+2 q_{1}\left(\vec{x}_{1}\right)+2^{2} q_{2}\left(\vec{x}_{2}\right)+2^{3} q_{3}\left(\vec{x}_{3}\right)
$$

where $q_{0}, q_{1}, q_{2}$ are of discriminant coprime to 2 and each $q_{i}$ consists of forms as in (62). For $m=4$ Rouse [45] shows that the existence of one good solution implies that there at least $2^{7}$ good solutions modulo 8 by enumerating all possibilities of the 2-adic Jordan decomposition. As a direct consequence, we get $\beta_{2}(Q, n) \geqslant \frac{1}{16}$ if $\operatorname{dim} q_{0}+\operatorname{dim} q_{1} \leqslant 4$ and there is a good solution. Indeed, $\vec{x}_{3}$ can be chosen arbitrarily and for $\vec{x}_{2}$ we only need to distinguish between $q_{2}\left(\vec{x}_{2}\right) \equiv 0(\bmod 2)$ and $q_{2}\left(\vec{x}_{2}\right) \equiv 1(\bmod 2)$ which costs us at most three-quarters of the solutions. If $\operatorname{dim} q_{0}+\operatorname{dim} q_{1}>4$ we apply [63, Theorem 4.1]. Let $d(k)$ and $\delta(k)$ denote the functions from [63, (4.3)]. Then, $d(1) \leqslant 0$ if $\delta(1) \neq 0, d(2) \leqslant-2$ if $\delta(2) \neq 0$ and $d(k) \leqslant 5 / 2-2 k$ which implies that $\beta_{2}(Q, n) \geqslant \frac{1}{4}$.

Bad type solutions only appear if $p \mid n$. We decompose $q$ into

$$
q_{0}\left(\vec{x}_{0}\right)+p q_{1}\left(\vec{x}_{1}\right)+p^{2} q_{2}\left(\vec{x}_{2}\right)
$$

where $q_{0}, q_{1}$ are of discriminant coprime to $p$ and $q_{0}, q_{1}, q_{2}$ consist of forms as in (62). For type I, we reduce the congruence by $p$; so instead of counting the solutions of

$$
q_{0}\left(\vec{x}_{0}\right)+p q_{1}\left(\vec{x}_{1}\right)+p^{2} q_{2}\left(\vec{x}_{2}\right) \equiv n\left(\bmod p^{t}\right)
$$

we use that $\vec{x}_{0} \equiv 0(\bmod p)$ and consider

$$
p q_{0}\left(\vec{x}_{0}\right)+q_{1}\left(\vec{x}_{1}\right)+p q_{2}\left(\vec{x}_{2}\right) \equiv n p^{-1}\left(\bmod p^{t-1}\right) .
$$

By definition of type I this congruence has solutions of good type. The corresponding lift has multiplicity $p^{\operatorname{dim} q_{1}+\operatorname{dim} q_{2}}$, since we are free to choose $\vec{x}_{1}$ and $\vec{x}_{2}$.

For type II, we reduce by $p^{2}$, thus, we consider

$$
q_{0}\left(\vec{x}_{0}\right)+p q_{1}\left(\vec{x}_{1}\right)+q_{2}\left(\vec{x}_{2}\right) \equiv n p^{-2}\left(\bmod p^{t-2}\right) .
$$

In this case, we can choose $\vec{x}_{2}$ freely, and for $\vec{x}_{0}, \vec{x}_{1}$, we have $p$ choices for each entry, so the multiplicity is

$$
p^{\operatorname{dim} q_{0}+\operatorname{dim} q_{1}+2 \operatorname{dim} q_{2}} .
$$

We apply these maps consecutively until we obtain a congruence that possesses a good type solution. We have shown the following:

Lemma 20. Let $p$ be odd and assume that $q(x) \equiv n\left(\bmod p^{t}\right), t \geqslant 1$ has a solution of good type. Then,

$$
\beta_{p}(Q, n) \geqslant 1-\frac{1}{p}-\frac{2}{p^{2}}
$$


while for a solution bad type I we have

$$
\beta_{p}(Q, n) \geqslant p^{1-\operatorname{dim} q_{0}}\left(1-\frac{1}{p}-\frac{2}{p^{2}}\right)
$$

and for bad type II that

$$
\beta_{p}(Q, n) \geqslant p^{2-\operatorname{dim} q_{0}-\operatorname{dim} q_{1}}\left(1-\frac{1}{p}-\frac{2}{p^{2}}\right) .
$$

For $p=2$ and a good solution modulo $2^{t}, t \geqslant 3$ the same bounds hold provided that we substitute $\left(1-\frac{1}{p}-\frac{2}{p^{2}}\right)$ by $\frac{1}{16}$.

The lower bounds in the lemma above are not optimal but sufficient for our purposes. We say that $q$ satisfies $(\mathrm{SLC})$ for $n$ if there is a solution of $q(x) \equiv n\left(\bmod p^{t}\right)$ with $Q x \neq 0(\bmod p)$ for all odd $p$ and $t \geqslant 1$ as well as for $p=2$ and $t \geqslant 3$. This condition is equivalent to the existence of good solutions modulo $p^{t}$. As a consequence, we obtain that:

Corollary 21. Assume that $m \geqslant 4$ and $q(x)=n$ is soluble over $x \in \mathbb{Z}_{p}$ for all primes. If $q$ satisfies (SLC) for $n$ or $(n, N)=1$, we have that

$$
\prod_{p} \beta_{p}(n, Q) \gg(n N)^{-\epsilon},
$$

if $m=4$ and $n$ is primitively locally represented by $q$ we obtain

$$
\beta_{p}(n, Q) \gg \prod_{p \mid N} \max \left(p^{-\left\lfloor\frac{v_{p}(N)+1}{2}\right\rfloor}, p^{-\left\lfloor\frac{v_{p}(\operatorname{det} Q)}{2}\right\rfloor}\right)(n N)^{-\epsilon},
$$

and for $m \geqslant 5$ we get that

$$
\beta_{p}(n, Q) \gg \prod_{p \mid N} \max \left(p^{-v_{p}(N)}, p^{-\left\lfloor\frac{v_{p}(\operatorname{det} Q)-2}{m-4}\right\rfloor}\right)(n N)^{-\epsilon},
$$

Proof. Recall that a quadratic form in three variables with unitary discriminant is isotropic over $\mathbb{Z}_{p}$ if $p$ is odd. We decompose $q$ as in (63). If $\operatorname{dim} q_{0} \geqslant 3$, there is a solution of good type and if $\operatorname{dim} q_{1} \geqslant 3$ there is a solution of bad type I. Furthermore, bad type solutions of type I only exist if $p^{m-2} \mid \operatorname{det} Q$ and type II solutions if $p^{2 m-6} \mid \operatorname{det} Q$. More generally, if the smallest type II solution $m_{j}$ has coefficient $p^{\nu_{j}}$, then $p^{(m-4) \nu_{j}+2} \mid \operatorname{det} Q$. For $p=2$, any form with four variables and unitary discriminant is isotropic over $\mathbb{Z}_{2}$. Hence, if $\operatorname{dim} q_{0} \geqslant 4$, there is a good type solution, and if $\operatorname{dim} q_{1} \geqslant 4$ there is a bad type solution of type I. The bounds follow now from Lemma 20, (58) and (59). 
In the class of $Q$ there exists an element $Q^{\prime}$ that lives in the Siegel domain $\mathcal{S}\left(\frac{4}{3}, \frac{1}{2}\right)$, cf. [14, p. 259]. These matrices admit a decomposition

$$
Q^{\prime}=V^{T} D V
$$

where $V$ is a rational upper triangular matrix with ones on the diagonal and all other entries $\left|v_{i, j}\right| \leqslant \frac{1}{2}$ and $D$ is a rational diagonal matrix with entries $a_{1}, \ldots, a_{m} \in$ $\mathbb{Q}$ such that

$$
a_{1} \geqslant 1, \quad \text { and } \quad a_{i+1} \geqslant(3 / 4) a_{i} \text { for all } i=1, \ldots, m-1 .
$$

This construction of $a_{1}, \ldots, a_{m}$ with respect to $Q$ is not unique. However, any $a_{i}$ is (up to a constant depending on $m$ ) of the same size as the $i$-th successive minimum of $Q$, cf. [14, $\$ 12$ Theorem 4.1].

Lemma 22. Consider $0<Q \in M_{m}(\mathbb{Z})$ with even diagonal, with level $N$ and $a_{1}, \ldots, a_{n}$ as above. Then, we have

$$
\left(\frac{3}{4}\right)^{i-1} \leqslant a_{i} \leqslant\left(\frac{4}{3}\right)^{m-i} N
$$

for every $1 \leqslant i \leqslant N$. Furthermore, it holds

$$
\sum_{x \leqslant l} r(Q, x)^{2} \ll\left(1+\frac{l^{\frac{1}{2}}}{\sqrt{a_{1}}}+\frac{l}{\sqrt{a_{1} a_{2}}}+\ldots+\frac{l^{m-\frac{3}{2}} \sqrt{a_{1} a_{2}} \sqrt{a_{m}}}{\operatorname{det} Q}+\frac{l^{m-1} \sqrt{a_{1} a_{2}}}{\operatorname{det} Q}\right) l^{\epsilon}
$$

and

$\sum_{x \leqslant l} r\left(N Q^{-1}, x\right)^{2} \ll\left(1+\frac{l^{\frac{1}{2}} \sqrt{a_{m}}}{\sqrt{N}}+\ldots+\frac{l^{m-\frac{3}{2}} \operatorname{det} Q}{N^{m-\frac{3}{2}} \sqrt{a_{1}} \sqrt{a_{m} a_{m-1}}}+\frac{l^{m-1} \operatorname{det} Q}{N^{m-1} \sqrt{a_{m} a_{m-1}}}\right) l^{\epsilon}$.

Proof. Since $r(Q, n)=r\left(U^{T} Q U, n\right)$ for any $U \in \mathrm{GL}_{m}(\mathbb{Z})$ we may assume that $Q \in \mathcal{S}\left(\frac{4}{3}, \frac{1}{2}\right)$. Since $Q=V^{T} D V$ for $V$ and $D$ as above, we obtain that

$$
q(x)=\frac{1}{2} x^{T} Q x=\frac{a_{1}}{2}\left(x_{1}+v_{1,2} x_{2}+\ldots+v_{1, m} x_{m}\right)^{2}+\cdots+\frac{a_{m}}{2} x_{m}^{2} .
$$

We count the number of solutions of $q(x)=n$. For $x_{3}, \ldots, x_{m}$ there are at most

$$
\left(2 \sqrt{\frac{n}{a_{3}}}+1\right) \cdots\left(2 \sqrt{\frac{n}{a_{m}}}+1\right)
$$

choices. After that, we are left with a binary quadratic form that has $\mathcal{O}\left(n^{\epsilon}\right)$ solutions. This yields

$$
r(Q, n) \ll_{m}\left(1+\frac{n^{1 / 2}}{\sqrt{a_{3}}}+\ldots+\frac{n^{\frac{m}{2}-1}}{\sqrt{a_{3} \cdots a_{m}}}\right) n^{\epsilon} .
$$


If we want to count all solutions of $q(x) \leqslant l$, we proceed as before, but bound the number of solutions for $x_{1}$ and $x_{2}$ by

$$
\left(2 \sqrt{\frac{l}{a_{1}}}+1\right) \text { and }\left(2 \sqrt{\frac{l}{a_{2}}}+1\right) .
$$

Thus, we obtain

$$
\sum_{x \leqslant l} r(Q, x) \ll 1+\max _{1 \leqslant j \leqslant m} \frac{l^{\frac{j}{2}}}{\sqrt{a_{1}} \cdots \sqrt{a_{j}}} .
$$

The first claim now follows from these two bounds and $a_{1} \cdots a_{m}=\operatorname{det} Q$.

Next, we consider the quadratic form corresponding to $N Q^{-1}$. It holds that

$$
N Q^{-1}=N\left(V^{T} D V\right)^{-1}=V^{-1}\left(N D^{-1}\right) V^{-T} .
$$

The diagonal entries of $N D^{-1}$ are

$$
\frac{N}{a_{1}}, \ldots, \frac{N}{a_{m}} \text { with } \quad \frac{N}{a_{i}} \geqslant \frac{3}{4} \frac{N}{a_{i+1}} .
$$

The lowest diagonal entry $\frac{N}{a_{m}}$ of $D$ equals the lower right entries of $N Q^{-1}$ since $V^{-1}$ is an upper and $V^{-T}$ a lower diagonal matrix with ones on the diagonal. Hence, $\frac{N}{a_{m}} \in \mathbb{Z}$ and thus $a_{m} \leqslant N$. If we combine this with $(64)$, we obtain the bounds for $a_{i}$ stated in the lemma.

Furthermore, it holds that

$$
\begin{aligned}
\tilde{q}(x) & :=\frac{1}{2} x^{T} N Q^{-1} x=\frac{1}{2} x^{T} V^{-1}\left(N D^{-1}\right) V^{-T} x \\
& =\frac{N}{2 a_{1}} x_{1}^{2}+\ldots+\frac{N}{2 a_{m}}\left(w_{1, m} x_{1}+\ldots+w_{m-1, m} x_{m-1}^{2}+x_{m}\right)^{2},
\end{aligned}
$$

where $w_{i j}$ are the entries of $V^{-1}$. These values $\left|w_{i j}\right|$ are bounded from above by a constant. To see this, write $V=I+M$ for the $m \times m$ identity $I$ and a nilpotent matrix $M$. A simple calculation shows that $V^{-1}=(I+M)^{-1}=I+\sum_{k=1}^{m-1}(-1)^{k} M^{k}$.

Next, we count solutions of $\tilde{q}(x)=n$. For $x_{1}, \ldots x_{m-2}$ there are

$$
\left(2 \sqrt{n \cdot \frac{a_{1}}{N}}+1\right) \cdots\left(2 \sqrt{n \cdot \frac{a_{m-2}}{N}}+1\right)
$$

choices and $\mathcal{O}\left(n^{\epsilon}\right)$ for $x_{m-1}$ and $x_{m}$. It follows that

$$
r\left(N Q^{-1}, n\right) \ll\left(1+\frac{n^{1 / 2} \sqrt{a_{m-2}}}{\sqrt{N}}+\ldots+\frac{n^{\frac{m}{2}-1} \sqrt{a_{1} \cdots a_{m-2}}}{N^{\frac{m}{2}-1}}\right) n^{\epsilon} .
$$


Furthermore, we obtain by the same argument as above that

$$
\sum_{x \leqslant l} r\left(N Q^{-1}, x\right) \ll 1+\max _{1 \leqslant j \leqslant m} l^{\frac{j}{2}} \cdot \frac{\sqrt{a_{m-j+1}} \cdots \sqrt{a_{m}}}{N^{\frac{j}{2}}} .
$$

Combining these bounds yields the second claim.

To compute the inner product of the cuspidal part of the theta series, we require a transformation formula for the theta series. For simplicity, we omit the weight $\frac{m}{2}$ in the slash operator $\mid[\varrho]_{\frac{m}{2}}$.

Lemma 23. Let $\varrho=\left(\begin{array}{ll}a & b \\ c & d\end{array}\right) \in \mathrm{SL}_{2}(\mathbb{Z})$ with $c \neq 0$ and $l=(N, c)$. Then,

$$
\theta(Q, z) \mid[\varrho]=\left(\operatorname{det} Q_{l}\right)^{-\frac{1}{2}} \sum_{x \in \mathbb{Z}^{m}} \alpha(x, \varrho, Q) e\left(\frac{1}{2} x^{T} D Q_{l}^{-1} x z\right),
$$

where $|\alpha(x, \rho, Q)|=1, D$ is a diagonal matrix of determinant $\left(\operatorname{det} Q, l^{m}\right)$ and $Q_{l}$ is of level $\frac{N}{l}$ and determinant $\frac{\operatorname{det} Q}{\left(\operatorname{det} Q, l^{m}\right)}$.

Remark. The matrix $Q_{l}$ is explicitly constructed in the proof below and is given by $Q_{l}=D^{-1} V^{T} Q V$ for $D$ as above and a matrix $V \in \mathrm{SL}_{m}(\mathbb{Z})$.

Proof. We factorize

$$
\varrho=\left(\begin{array}{cc}
1 & a / c \\
& 1
\end{array}\right)\left(\begin{array}{cc} 
& -1 / c \\
c &
\end{array}\right)\left(\begin{array}{cc}
1 & d / c \\
& 1
\end{array}\right)
$$

and obtain by the transformation formula for the generalized theta series, cf. [55, p. 575], that

$$
\theta(Q, z) \mid[\varrho]=\sum_{x \in \mathbb{Z}^{m}} \alpha(x, Q, \varrho) e\left(\frac{1}{2} x^{T} Q^{-1} x z\right)
$$

where

$$
\alpha(x, Q, \varrho)=\frac{\zeta}{c^{\frac{m}{2}} \sqrt{\operatorname{det} Q}} e\left(\frac{d}{2 c} x^{T} Q^{-1} x\right) \sum_{u \in\left(\mathbb{Z} / c_{j} \mathbb{Z}\right)^{m}} e\left(\frac{1}{c} u^{T} x+\frac{a}{2 c} u^{T} Q u\right)
$$

and $|\zeta|=1$. Then,

$$
\begin{aligned}
\left|\sum_{u} \ldots\right|^{2} & =\sum_{u \in(\mathbb{Z} / c \mathbb{Z})^{m}} \sum_{v \in(\mathbb{Z} / c \mathbb{Z})^{m}} e\left(\frac{a}{2 c}\left(u^{T} Q u-v^{T} Q v\right)+\frac{1}{c}(u-v)^{T} x\right) \\
& =\sum_{w \in(\mathbb{Z} / c \mathbb{Z})^{m}} e\left(\frac{a}{2 c} w^{T} Q w+\frac{1}{c} w^{T} x\right) \sum_{v \in(\mathbb{Z} / c \mathbb{Z})^{m}} e\left(\frac{a}{c} v^{T} Q w\right) .
\end{aligned}
$$


We write $c=\tilde{c} 2^{t}$ with odd $\tilde{c}$ and decompose the $v$ and $w$ sums accordingly. As $\tilde{c}$ is odd, we can find $M \in \mathrm{GL}_{m}(\mathbb{Z} / \tilde{c} \mathbb{Z})$ such that $M^{T} Q M$ is congruent to a diagonal matrix $D \bmod \tilde{c}$ with entries $q_{1} \ldots, q_{m}$. Hence, substituting $v$ by $M v$ and $w$ by $M w$ we obtain

$$
\begin{aligned}
\sum_{w \in\left(\mathbb{Z} / \tilde{c}_{j} \mathbb{Z}\right)^{m}} e\left(\frac{a}{2 \tilde{c}} w^{T} Q w+\frac{1}{\tilde{c}} w^{T} x\right) \sum_{v \in(\mathbb{Z} / \tilde{c} \mathbb{Z})^{m}} e\left(\frac{a}{\tilde{c}} v^{T} Q w\right) \\
=\prod_{1 \leqslant i \leqslant m} \sum_{w_{i} \bmod \tilde{c}} e\left(\frac{a \overline{2} q_{i} w_{i}^{2}}{\tilde{c}}+\frac{w_{i} m_{i}^{T} x}{\tilde{c}}\right) \sum_{v_{i} \bmod \tilde{c}} e\left(\frac{a q_{i} v_{i} w_{i}}{\tilde{c}}\right),
\end{aligned}
$$

where $m_{i}$ denotes the $i$-th row of $M$ and $v_{i}, w_{i}$ the $i$-th entry of $v, w$. Set $\tilde{l}_{i}=\left(q_{i}, \tilde{c}\right)$ and $\tilde{l}=\left(\tilde{l}_{1}, \ldots, \tilde{l}_{m}\right)^{T}$. By applying the orthogonality relation and recalling that $(a, \tilde{c})=1$, the previous display equals

$$
\tilde{c}^{m} \prod_{1 \leqslant i \leqslant m} \sum_{w_{i} \bmod \tilde{l}_{i}} e\left(\frac{w_{i} m_{i}^{T} x_{i}}{\tilde{l}_{i}}\right)= \begin{cases}\tilde{c}^{m}\left(\operatorname{det} Q, \tilde{c}^{m}\right) & \text { if } M^{T} x \equiv 0(\bmod \tilde{l}), \\ 0 & \text { otherwise. }\end{cases}
$$

Over $\mathbb{Z}_{2}$ we cannot diagonalize every quadratic form, but we can decompose it into a sum of diagonal terms and multiples of the binary quadratic forms $x y$ and $x^{2}+x y+y^{2}$, cf. [28, Theorem 33]. Hence, there is $\tilde{M} \in \mathrm{GL}_{m}\left(\mathbb{Z} / 2^{t} \mathbb{Z}\right)$ such that $q(x)=\frac{1}{2} x^{T} \tilde{M}^{T} Q M x$ satisfies

$$
q \equiv 2^{\nu_{1}} g_{1}+\ldots+2^{\nu_{r}} g_{r}+2^{\tilde{\nu}_{1}} d_{1}+\ldots+2^{\tilde{\nu}_{s}} d_{s} \quad\left(\bmod 2^{t}\right)
$$

for $2 r+s=m, \nu_{i}, \tilde{\nu}_{i} \leqslant t$, binary quadratic forms $g_{i}$ given by matrices

$$
\left(\begin{array}{cc}
\alpha_{i} & 1 \\
1 & \alpha_{i}
\end{array}\right) \text { with } \alpha_{i} \equiv 0 \text { or } \alpha_{i} \equiv 2 \quad\left(\bmod 2^{t}\right)
$$

and diagonal forms $d_{i}$ with discriminant coprime to 2 . The $d_{i}$ can be treated as above. For the binary quadratic form $g_{1}$ we need to evaluate:

$$
\begin{aligned}
\sum_{w_{1}, w_{2}\left(\bmod 2^{t}\right)} e & \left(\frac{a\left(\alpha_{1} w_{1}^{2}+2 w_{1} w_{2}+\alpha_{1} w_{2}^{2}\right)}{2^{t-\nu_{1}+1}}+\frac{w_{1} \tilde{m}_{1}^{T} x_{1}+w_{2} \tilde{m}_{2}^{T} x_{2}}{2^{t}}\right) \\
& \times \sum_{v_{1}\left(\bmod 2^{t}\right)} e\left(\frac{a v_{1}\left(\alpha_{1} w_{1}+w_{2}\right)}{2^{t-\nu_{1}}}\right) \sum_{v_{2}\left(\bmod 2^{t}\right)} e\left(\frac{a v_{2}\left(w_{1}+\alpha_{1} w_{2}\right)}{2^{t-\nu_{1}}}\right) .
\end{aligned}
$$

The latter two sums vanish unless

$$
\alpha w_{1}+w_{2} \equiv 0\left(\bmod 2^{t-\nu_{1}}\right) \text { and } w_{1}+\alpha w_{2} \equiv 0\left(\bmod 2^{t-\nu_{1}}\right) .
$$

This implies that $w_{1}, w_{2} \equiv 0\left(\bmod 2^{t-\nu_{1}}\right)$. Hence, display $(68)$ equals

$2^{2 t} \sum_{w_{1}, w_{2}\left(\bmod 2^{\nu_{1}}\right)} e\left(\frac{w_{1} \tilde{m}_{1}^{T} x_{1}+w_{2} \tilde{m}_{2}^{T} x_{2}}{2^{\nu_{1}}}\right)= \begin{cases}2^{2 t+2 \nu_{1}} & \text { if } \tilde{m}_{1}^{T} x_{1} \equiv \tilde{m}_{2}^{T} x_{2} \equiv 0 \bmod 2^{\nu_{1}}, \\ 0 & \text { otherwise. }\end{cases}$ 
The rest works analogously. Let $D$ denote the diagonal matrix with entries $l_{i}$ given by

$$
l_{i}=\tilde{l}_{i} 2^{\nu(i)} \text { with } \nu(i)= \begin{cases}\nu_{\left\lfloor\frac{i+1}{2}\right\rfloor} & \text { if } i \leqslant 2 r \\ \tilde{\nu}_{i-2 r} & \text { if } i>2 r .\end{cases}
$$

By construction, we have $\operatorname{det} D=l_{1} \cdots l_{m}=\left(\operatorname{det} Q, c^{m}\right)$. We choose $V \in \mathrm{GL}_{m}(\mathbb{Z})$ with $V \equiv M(\bmod \tilde{c})$ and $V \equiv \tilde{M}\left(\bmod 2^{t}\right)$. The computation above gives the following congruence condition for $x$ :

$$
V^{T} x \equiv 0\left(\bmod D \mathbb{Z}^{m}\right)
$$

Thus, (66) equals

$$
\begin{aligned}
& \zeta \frac{\sqrt{\left(\operatorname{det} Q, c^{m}\right)}}{\sqrt{\operatorname{det} Q}} \sum_{\substack{x \in \mathbb{Z}^{m} \\
V^{T} x \equiv 0\left(\bmod D \mathbb{Z}^{m}\right)}} e\left(\frac{1}{2}\left(V^{T} x\right)^{T}\left(V^{T} Q V\right)^{-1} V x\left(z+\frac{d}{c}\right)\right) \\
& =\zeta \frac{\sqrt{\left(\operatorname{det} Q, c^{m}\right)}}{\sqrt{\operatorname{det} Q}} \sum_{x \in \mathbb{Z}^{m}} e\left(\frac{1}{2} x^{T} D\left(V^{T} Q V\right)^{-1} D x\left(z+\frac{d}{c}\right)\right) .
\end{aligned}
$$

Note that both $D$ and $V$ only depend on $l=(N, c)$ and not on the specific value of c. By construction $Q_{l}:=D^{-1} V^{T} Q V$ is a positive, integer matrix of level $\frac{N}{(N, c)}=\frac{N}{l}$. The claim follows by setting $\alpha(x, \varrho, \tilde{Q})=\zeta e\left(\frac{d}{2 c}\left(x^{T} D \tilde{Q}^{-1} x\right)\right.$.

We improve the estimates in [4, Lemma 4.2] and [5, Lemma 2.3] by means of Lemma 22 and 23 .

Lemma 24. Consider a positive, integral, primitive quadratic form in $m \geqslant 3$ variables given by $Q$. If $f(z)=\theta(Q, z)-\theta(\operatorname{gen} Q, Z)$ and $q(x)=\sum_{i} a_{i} x_{i}^{2}$ with $a_{i} \leqslant a_{j}$ for $i<j$, then

$$
\langle f, f\rangle \ll\left(\frac{N^{\frac{m}{2}}}{\left(\operatorname{det} Q, N^{\frac{m}{2}}\right)}+\frac{N}{\sqrt{a_{m} a_{m-1}}}\right) N^{\epsilon} .
$$

More general, if $Q, Q^{\prime}$ are in the same genus and $\tilde{f}(z)=\theta(Q, z)-\theta\left(Q^{\prime}, z\right)$ it holds that

$$
\langle\tilde{f}, \tilde{f}\rangle \ll\left(\frac{N^{\frac{m}{2}}}{\left(\operatorname{det} Q, N^{\frac{m}{2}}\right)}+\frac{N}{(\operatorname{det} Q)^{\frac{1}{m}}}\right) N^{\epsilon} .
$$

Proof. First, note that $f$ is a cusp form since the value of $\theta(Q, z)$ at each cusp depends only on a genus-invariant term, cf. Corollary 32 . Set $\mu=\left[\mathrm{SL}_{2}(\mathbb{Z}): \Gamma_{0}(N)\right]$ and $\tilde{\mu}=\left[\mathrm{SL}_{2}(\mathbb{Z}): \Gamma(N)\right]$ and recall that

$$
\frac{\tilde{\mu}}{\mu}=N^{2} \prod_{p \mid N}\left(1-\frac{1}{p}\right) .
$$


We obtain

$$
\left.\langle f, f\rangle=\int_{\Gamma_{0}(N) \backslash \mathbb{H}}|f(z)|^{2} y^{\frac{m}{2}-2} d x d y=\frac{\mu}{\tilde{\mu}} \sum_{j=1}^{\tilde{\mu}} \int_{\mathrm{SL}_{2}(\mathbb{Z}) \backslash \mathbb{H}}|f|\left[\tau_{j}\right]\right)\left.(z)\right|^{2} y^{k-2} d x d y
$$

for a set of coset representatives $\tau_{i}$ of $\Gamma(N) \backslash \mathrm{SL}_{2}(\mathbb{Z})$. On this set, we define an equivalence relation

$$
\tau_{i} \sim \tau_{j} \Leftrightarrow \tau_{i} \in \Gamma(N) \tau_{j} T \quad \text { for } T=\left\{\left(\begin{array}{ll}
1 & x \\
& 1
\end{array}\right) \mid 0 \leqslant x \leqslant N-1\right\}
$$

and choose a subset $\left\{\varrho_{1}, \ldots, \varrho_{\tilde{\mu} / N}\right\}$ of representatives. Thus,

$$
\left.\langle f, f\rangle=\frac{\mu}{\tilde{\mu}} \sum_{j=1}^{\tilde{\mu} / N} \bigcup_{t \in T} \int_{t F}|f|\left[\varrho_{j}\right]\right)\left.(z)\right|^{2} y^{k-2} d x d y
$$

where $F$ is a fundamental domain of $\mathrm{SL}_{2}(\mathbb{Z}) \backslash \mathbb{H}$. Let

$$
\varrho_{j}=\left(\begin{array}{ll}
a_{j} & b_{j} \\
c_{j} & d_{j}
\end{array}\right) .
$$

By construction, the $\tilde{\mu} / N$ cusps $a_{j} / c_{j}$ are a complete set of representatives for $\Gamma(N)$ (with the convention that $1 / 0=\infty$ ). Recall that two rational numbers $a_{j} / c_{j}, a_{j}^{\prime}, c_{j}^{\prime}$ are $\Gamma(N)$-equivalent cusps if and only if $a \equiv a^{\prime}(\bmod N)$ and $c \equiv c^{\prime}(\bmod N)$, so in particular $(c, N)=\left(c^{\prime}, N\right)$. Hence, for $d \mid N$ exactly

$$
\varphi\left(\frac{N}{d}\right) \frac{\varphi(d)}{d} N
$$

of the $c_{j}$ satisfy $\left(c_{j}, N\right)=d$.

First, we estimate the contribution of the $\varphi(N)$ matrices $\varrho_{j}$ with $c_{j}=0$. In this case $f \mid\left[\varrho_{j}\right]=f$ and hence, these matrices contribute at most

$$
\frac{\mu}{\tilde{\mu}} \varphi(N) N \int_{\frac{1}{2}}^{\infty} \sum_{n=1}^{\infty}|r(Q, n)-r(\operatorname{gen} Q, n)|^{2} \exp (-4 \pi y) y^{\frac{m}{2}-2} d y \ll_{m} 1
$$

to $(69)$.

For $\varrho_{j}$ with $c_{j} \neq 0$, we apply Lemma 23 which yields the following Fourier expansion for $f \mid\left[\varrho_{j}\right]$ :

$$
\left(f \mid\left[\varrho_{j}\right]\right)(z)=\left(\operatorname{det} Q_{d}\right)^{-\frac{1}{2}} \sum_{n \geqslant 0} a_{d}(n) e\left(\frac{n z}{N_{d}}\right)
$$


where $N_{d}=\frac{N}{\left(N, c_{j}\right)}=\frac{N}{d}$ and $\left|a_{d}(n)\right| \leqslant r\left(S_{d}, n\right)+r\left(\right.$ gen $\left.S_{d}, n\right)$ for $S_{d}=N_{d} D_{d} Q_{d}^{-1}$ with $Q_{d}=V^{T} Q V D_{d}^{-1}$ as in Lemma 23. In particular, we have

$$
\operatorname{det} Q_{d}=\frac{\operatorname{det} Q}{\left(\operatorname{det} Q, d^{m}\right)} \quad \text { and } \quad \operatorname{det} S_{d}=\frac{N_{d}^{m}\left(\operatorname{det} Q, d^{m}\right)}{\operatorname{det} Q_{d}} \text {. }
$$

It follows that

$$
\begin{aligned}
\langle f, f\rangle & \ll 1+N \frac{\mu}{\tilde{\mu}} \sum_{d \mid N} \sum_{\left(c_{j}, N\right)=d}\left(\operatorname{det} Q_{d}\right)^{-1} \int_{\frac{1}{2}}^{\infty} \sum_{n \geqslant 1} a_{d}(n)^{2} \exp \left(\frac{-4 \pi y n}{N_{d}}\right) y^{\frac{m}{2}-2} d y \\
& \ll 1+\sum_{d \mid N} \frac{N_{d}}{\operatorname{det} Q_{d}} \sum_{n \geqslant 1} a_{d}(n)^{2}\left(\frac{N_{d}}{n}\right)^{\frac{m}{2}-1} \exp \left(\frac{n}{N_{d}}\right) .
\end{aligned}
$$

We truncate the $n$ sum at $n \ll N_{d}^{1+\epsilon}$. First, we consider the contribution of the genus theta series. By (61) we obtain that

$$
\frac{N_{d}^{\frac{m}{2}}}{\operatorname{det} Q_{d}} \sum_{n \ll N_{d}^{1+\epsilon}} \frac{r\left(\operatorname{gen} S_{d}, n\right)^{2}}{n^{\frac{m}{2}-1}} \ll N_{d}^{-\frac{m}{2}} \sum_{n \ll N_{d}^{1+\epsilon}} \frac{n^{\frac{m}{2}-1}(n, N)}{\left(\operatorname{det} Q, d^{m}\right)} \ll N^{\epsilon} .
$$

To estimate $r\left(S_{d}, n\right)$, we apply Lemma 22 . Following the construction of $a_{1}, \ldots, a_{m}$ just before this lemma we obtain tuples $a_{1}\left(S_{d}\right), \ldots, a_{m}\left(S_{d}\right)$ with respect to $S_{d}$ and $a_{1}\left(Q_{d}\right), \ldots, a_{m}\left(Q_{d}\right)$ with respect to $Q_{d}$. It follows that

$$
\begin{aligned}
\frac{N_{d}^{\frac{m}{2}}}{\operatorname{det} Q_{d}} & \sum_{n \ll N_{d}^{1+\epsilon}} \frac{r\left(S_{d}, n\right)^{2}}{n^{\frac{m}{2}-1}} \\
& \ll\left(\frac{N_{d}^{\frac{m}{2}}}{\operatorname{det} Q_{d}}+\ldots+\frac{\sqrt{a_{1}\left(S_{d}\right) a_{2}\left(S_{d}\right)} \sqrt{a_{m}\left(S_{d}\right)}}{\sqrt{N_{d}}\left(\operatorname{det} Q, d^{m}\right)}+\frac{\sqrt{a_{1}\left(S_{d}\right) a_{2}\left(S_{d}\right)}}{\left(\operatorname{det} Q, d^{m}\right)}\right) N_{d}^{\epsilon} \\
& \ll\left(\frac{N_{d}^{\frac{m}{2}}}{\operatorname{det} Q_{d}}+\frac{N_{d}}{a_{m}\left(Q_{d}\right) a_{m-1}\left(Q_{d}\right)}\right) N_{d}^{\epsilon} .
\end{aligned}
$$

If $Q$ is diagonal, then $Q_{d}$ is also diagonal and we can arrange the coefficients by size and set $a_{i}\left(Q_{d}\right)$ as the $i$-th entry. Since $a_{i}(Q) \mid N$, we may decompose $a_{i}:=a_{i}(Q)$ as $\left(a_{i}, N_{d}\right)\left(a_{i}, d\right)$. By definition of $Q_{d}$ we have $a_{m}\left(Q_{d}\right) a_{m-1}\left(Q_{d}\right) \geqslant\left(a_{m}, N_{d}\right)\left(a_{m-1}, N_{d}\right)$. Thus,

$$
\frac{N_{j}}{\sqrt{a_{m}\left(Q_{d}\right) a_{m-1}\left(Q_{d}\right)}} \leqslant \frac{N_{d}}{\sqrt{\left(a_{m}, N_{d}\right)\left(a_{m-1}, N_{d}\right)}} \frac{d}{\sqrt{\left(a_{m}, d\right)\left(a_{m-1}, d\right)}}=\frac{N}{\sqrt{a_{m} a_{m-1}}} .
$$

In this case, the inner product is bounded by

$$
\langle f, f\rangle \ll \sum_{d \mid N}\left(\frac{N_{d}^{\frac{m}{2}}}{\operatorname{det} Q_{d}}+\frac{N}{\sqrt{a_{m} a_{m-1}}}+1\right) N^{\epsilon} \ll\left(\frac{N^{\frac{m}{2}}}{\left(\operatorname{det} Q, N^{\frac{m}{2}}\right)}+\frac{N}{\sqrt{a_{m} a_{m-1}}}\right) N^{\epsilon} .
$$


If $q$ is not diagonal, we simply estimate $a_{m}\left(Q_{j}\right) a_{m-1}\left(Q_{j}\right) \geqslant\left(\operatorname{det} Q_{j}\right)^{\frac{2}{m}}$. As a consequence, we get

$$
\langle f, f\rangle \ll\left(\frac{N^{\frac{m}{2}}}{\left(\operatorname{det} Q, N^{\frac{m}{2}}\right)}+\frac{N}{(\operatorname{det} Q)^{\frac{1}{m}}}\right) N^{\epsilon} .
$$

This bound is a significant improvement to previous results. For $m \geqslant 3$ it is known by [47] that

$$
\langle f, f\rangle \ll N^{m-2+\epsilon}+N^{\frac{m-3}{2}} \sqrt{\operatorname{det} Q}+(\operatorname{det} Q)^{1-\frac{1}{m}}
$$

and for $m=4$ by [45] that

$$
\langle f, f\rangle \ll N^{\frac{3}{2}+\epsilon}(\operatorname{det} Q)^{\frac{1}{4}}+N^{2+\epsilon} .
$$

An application of Lemma 24 is the representation by squares of almost primes. While every number $n \equiv 5(\bmod 24)$ can be expressed by the sum of five squares, it is not possible to prove an analogous result for four or less squares by current technology. However, using a vector sieve, Blomer and Brüdern, [10] [7], [5] obtain similar findings for almost primes. In the case of three squares, the main input is [7, Theorem 4.2] where for $n \equiv 3(\bmod 8)$ and the quadratic form $q_{d}=d_{1}^{2} x_{1}^{2}+$ $d_{2}^{2} x_{2}^{2}+d_{3}^{2} x_{3}^{2}=\frac{1}{2} x^{T} Q_{d} x$, the error term is given by

$$
R_{3}(\theta)=\sum_{d_{1}, d_{2}, d_{3} \leqslant n^{\theta}} \mu\left(d_{1}\right) \mu\left(d_{2}\right) \mu\left(d_{3}\right)\left|r\left(Q_{d}, n\right)-r\left(\operatorname{gen} Q_{d}, n\right)\right| .
$$

The aim is to maximize $\theta$ such that $R_{3}(\theta) \ll n^{\frac{1}{2}-\epsilon}$. Let $N$ denote the level of $Q_{d}$. First, we use a local argument from Blomer [5, p. 14] which allows us to assume that $(n, N)$ is relatively small. Then, we apply [60, Theorem 1] and Lemma 24. This gives

$$
\begin{aligned}
R_{3}(\theta) & \ll \sum_{d_{1}, d_{2}, d_{3} \leqslant n^{\theta}} n^{\frac{1}{4}}\left(\frac{n^{3 / 14}}{N^{\frac{1}{7}}}+\frac{n^{\frac{3}{16}}}{N^{\frac{1}{16}}}+1\right)\left(\frac{N^{\frac{3}{4}}}{\sqrt{\operatorname{det} Q}}+\frac{\sqrt{N}}{(\operatorname{det} Q)^{\frac{1}{6}}}\right)(n N)^{\epsilon} \\
& \ll n^{\frac{1}{4}} \sum_{\substack{d_{1}, d_{2}, d_{3} \leqslant n^{\theta} \\
\text { (n) }}}\left(n^{\frac{13}{28}}\left(d_{1} d_{2} d_{3}\right)^{\frac{2}{3}-\frac{2}{7}}+n^{\frac{7}{16}}\left(d_{1} d_{2} d_{3}\right)^{\frac{2}{3}-\frac{1}{8}}\right)(n N)^{\epsilon} \\
& \ll n^{\frac{13}{28}+\left(4+\frac{1}{7}\right) \theta}(n N)^{\epsilon}+n^{\frac{7}{16}+\left(4+\frac{5}{8}\right) \theta}(n N)^{\epsilon} .
\end{aligned}
$$

We conclude that $R_{3}(\theta) \ll n^{\frac{1}{2}-\epsilon}$ is satisfied as long as $\theta<\frac{1}{116}$. If we insert this bound into [13, 3.50], where the variable $\theta$ is called $\eta$, we obtain that every large integer $n \equiv 3(\bmod 24)$ with $5 \nmid n$ is represented by three almost primes with each at most 67 prime divisors. 
For four squares, the main input is [10, Theorem 3] which shows that

$$
R_{4}(\theta)=\sum_{d_{1}, d_{2}, d_{3} \leqslant n^{\theta}} \mu\left(d_{1}\right) \mu\left(d_{2}\right) \mu\left(d_{3}\right) \mu\left(d_{3}\right) \mid r\left(Q_{d}, n\right)-r\left(\text { gen } Q_{d}, n\right) \mid \ll n^{1-\epsilon}
$$

for the quadratic form $q_{d}=d_{1}^{2} x_{1}^{2}+d_{2}^{2} x_{2}^{2}+d_{3}^{2} x_{3}^{2}+d_{4}^{2} x_{4}^{2}=\frac{1}{2} x^{T} Q_{d} x$ and $\theta<\frac{1}{22}$. Let $N$ denote the level of $q_{d}$. By the Petersson formula and Lemma 24, we obtain

$$
\begin{aligned}
R_{4}(\theta) & \ll \sum_{d_{1}, d_{2}, d_{3} \leqslant n^{\theta}} n^{\frac{1}{2}}\left(1+\frac{n^{\frac{1}{4}}(n, N)^{\frac{1}{4}}}{N^{\frac{1}{2}}}\right)\left(\frac{N}{\sqrt{\operatorname{det} Q}}+\frac{\sqrt{N}}{\sqrt{d_{3} d_{4}}}\right) \\
& \ll n^{8 \theta} n^{\frac{1}{2}}+n^{\frac{3}{4}}\left(\sum_{d \leqslant n^{\theta}}\left(n, d^{2}\right)^{\frac{1}{4}}\right)^{4} \ll n^{8 \theta+\frac{1}{2}}+n^{4 \theta+\frac{3}{4}}
\end{aligned}
$$

Hence, if $0<\theta<\frac{1}{16}$, then $R_{4}(\theta) \ll n^{1-\epsilon}$ which improves [10, Theorem 3].

Another application is due to Golubeva [21] who shows that every even and sufficiently large $n \equiv 1(\bmod 5)$ can be represented by two squares, one cube, two fourth and one sixteenth power. Therefore, she shows that

$$
n=x_{1}^{2}+x_{2}^{2}+6 P x_{3}^{2},
$$

is solvable if $n$ is larger than a small power of $P$. By the lemma above, the cuspidal part $f$ of $\theta(Q, z)$ satifies $\|f\| \ll P^{1 / 4+\epsilon}$ which is sufficient to show that $r(n, Q) \geqslant 1$ if $n>P^{17+\epsilon}$.

To conclude this section, we determine a lower bound for $n$ with respect to $Q$. To bound the Fourier coefficients of $f(z)=\theta(Q, z)-\theta($ gen $Q, z)=\sum_{n \geqslant 1} a(n) e(n z)$, we apply the lemma above and either Deligne's proof of the Ramanujan-Petersson conjecture or the Petersson formula, cf. [27, Corollary 14.24]. This gives for even $m$ the following bounds

$$
\begin{aligned}
a(n) & \ll\|f\| n^{\frac{m}{4}-\frac{1}{2}} \min \left(N^{\frac{1}{2}+\epsilon}, 1+n^{\frac{1}{4}}(n, N)^{\frac{1}{4}} N^{-\frac{1}{2}}\right) \\
& \ll n^{\frac{m}{4}-\frac{1}{2}}\left(\frac{N^{\frac{m}{4}}}{\left(\sqrt{\operatorname{det} Q}, N^{\frac{m}{4}}\right)}+\frac{N^{\frac{1}{2}}}{\sqrt[4]{a}}\right) \min \left(N^{\frac{1}{2}+\epsilon}, 1+\frac{n^{\frac{1}{4}}(n, N)^{\frac{1}{4}}}{N^{\frac{1}{2}}}\right)
\end{aligned}
$$

and for odd $m$ that

$$
a(n) \ll\|f\| n^{\frac{m}{4}-\frac{1}{2}+\epsilon}\left(1+\frac{n^{\frac{1}{4}}(n, N)^{\frac{1}{4}}}{N^{\frac{1}{2}-\epsilon}}\right) .
$$

If $n=t v^{2} w^{2}$ with $t$ squarefree and $v \mid N^{\infty}$ not too large, one can get additional saving by applying [59, Theorem 1].

Combining the results in Lemma 24, (70) and Corollary 21 shows that:

Corollary 25. Consider an integral positive quadratic form $q(x)=\frac{1}{2} x^{T} Q x$ in four variables. If $q$ is diagonal, let a denote the product of the two largest coefficients; 
otherwise, we set $a=\sqrt{\operatorname{det} Q}$. Then, $q(x)=n$ is soluble provided that $q$ satisfies (SLC) for $n$ and

$$
n \gg\left(N^{2}(n, N)+\min \left(\frac{(n, N)(\operatorname{det} Q)^{2}}{a}, \frac{N^{2} \operatorname{det} Q}{\sqrt{a}}\right)\right) N^{\epsilon}
$$

or if $n$ is primitively locally represented by $q$ and

$$
n \gg\left(N^{3}+\frac{N^{2} \operatorname{det} Q}{\sqrt{a}}\right) N^{\epsilon} \min (N \tilde{N}, \operatorname{det} Q)
$$

where $N=\tilde{N} l^{2}$ with $\tilde{N}$ squarefree.

This is a significant improvement of previous results as for example [45, Theorem 1]. For quadratic forms in $m$ variables with $m \geqslant 5$, we get a similar result:

Corollary 26. Consider an integral positive quadratic form $q(x)=\frac{1}{2} x^{T} Q x$ in $m$ variables with $m \geqslant 5$ and set a as the product of the two largest coefficients if $q$ is diagonal and $a=(\operatorname{det} Q)^{\frac{2}{m}}$ otherwise. Then, $q(x)=n$ is soluble provided that $n$ is locally represented by $q$ and

$$
n \gg\left(N^{1+\frac{2}{m-3}}+\frac{\operatorname{det} Q \sqrt{N}}{\sqrt{a}}\right)^{\frac{2}{m-3}} f(N)
$$

where

$$
f(N)= \begin{cases}N^{\epsilon} & \text { if q satisfies (SLC) for } n \\ \min \left(N^{1+\epsilon},(\operatorname{det} Q)^{\frac{1-\delta}{m-4}}\right)^{\frac{4}{m-3}} & \text { otherwise }\end{cases}
$$

and any $\delta>0$ with $(\operatorname{det} Q)^{\delta} \leqslant \prod_{p \mid \operatorname{det} Q}\left(p^{2}, \operatorname{det} Q\right)$. Furthermore $n$ is represented if $(n, N)=1$ and

$$
n \gg N^{1+\frac{2}{m-2}+\epsilon}+\left(\frac{\operatorname{det} Q}{\sqrt{a}}\right)^{\frac{2}{m-3}+\epsilon} .
$$

The size of $f(N)$ depends on the lower bounds for the $p$-adic densities in Corollary 21. If only small powers of primes divide $\operatorname{det} Q$, one can improve the minimum term in $f(N)$.

In the following sections, we frequently use the bound above for the cuspidal part of the theta series, however, only for prime level. To hold notation simple, we use $k=\frac{m}{2}$ and write the first term in the bound as $\frac{N^{k+\epsilon}}{\operatorname{det} Q}$. 


\section{Theta series and Klingen-Eisenstein series of degree two}

Consider two positive, integral quadratic forms in 2 and $m$ variables given by $2 T$ and $Q$. The aim of this section is to obtain an asymptotic formula for

$$
r(Q, T)=\left\{X \in M_{2 k, 2}(\mathbb{Z}) \mid T=\frac{1}{2} X^{T} Q X\right\}
$$

that is uniform in the level $N$ of $Q$. We tacitly assume that $Q$ is primitive which implies that the level of $N Q^{-1}$ is also $N$.

We start by reviewing the theory of Siegel modular forms (of degree two) and explain how to construct a basis for the space of Eisenstein series. Furthermore, we present various transformation formulas for the theta series of degree two. Then, we dive deep into the world of Kingen-Eisenstein series. To evaluate the Fourier coefficients, we express them as an integral. The first step is to decompose the matrices that appear in the sum of the Klingen-Eisenstein series into different parts. Then, by treating each of these components individually, we obtain an explicit formula for the Fourier coefficients.

To obtain an asymptotic formula of $r(Q, T)$ it remains to treat the cuspidal part of the theta series. After applying Kitaoka's equivalent of the Petersson formula we are left with estimating the Petersson inner product. To reduce the computation to the fundamental domain of $\operatorname{Sp}_{4}(\mathbb{Z}) \backslash \mathbb{H}_{2}$, it is essential to obtain transformation formulas for Klingen-Eisenstein and theta series with respect to a set of representatives in $\Gamma_{0}^{(2)}(N) \backslash \operatorname{Sp}_{4}(\mathbb{Z})$. This evaluation proves to be lengthy and technical. The final part of this chapter discusses several ways how to extend the results of Theorem 6 .

\subsection{Siegel modular forms and the space of Eisenstein series}

We start by introducing further notation. We set

$$
\operatorname{GSp}_{2 n}(\mathbb{R})=\left\{M \in \mathrm{GL}_{2 n}(\mathbb{R}) \mid M^{T} J_{2 n} M=\mu(M) J_{2 n}\right\} \quad \text { for } \quad J_{2 n}=\left(\begin{array}{cc} 
& -I_{n} \\
I_{n} &
\end{array}\right)
$$

and some $\mu(M) \in \mathrm{GL}_{1}(\mathbb{R})$. The kernel of $\mu: \mathrm{GSp}_{2 n} \rightarrow \mathrm{GL}_{1}$ is by definition $\mathrm{Sp}_{2 n}$. Note that $M=\left(\begin{array}{ll}A & B \\ C & D\end{array}\right) \in \operatorname{GSp}_{2 n}(\mathbb{R})$ if and only if $A^{T} D-C^{T} B=\mu(M) I_{n}$ and $A^{T} C$ and $B^{T} D$ are symmetric. By $\operatorname{GSp}_{2 n}(\mathbb{R})^{+}$we denote the subgroup of $\mathrm{GSp}_{2 n}(\mathbb{R})$ consisting of those elements $M$ with $\mu(M)>0$.

Let $\mathbb{H}_{n}=\left\{Z=X+I Y \in \operatorname{Mat}_{n}(\mathbb{C}) \mid Y>0\right\}$ be Siegel's upper half space of degree $n$. We let $M=\left(\begin{array}{cc}A & B \\ C & D\end{array}\right) \in \mathrm{GSp}_{2 n}^{+}(\mathbb{R})$ act on $\mathbb{H}_{n}$ by

$$
M Z=(A Z+B)(C Z+D)^{-1}
$$


and define for $k \in \mathbb{Z}$ an action of $\operatorname{GSp}_{2 n}^{+}(\mathbb{R})$ on functions $F: \mathbb{H}_{n} \rightarrow \mathbb{C}$ by

$$
\left(F \mid[M]_{k}\right)(Z)=\mu(M)^{k} j_{M}(Z)^{-k} F(M Z),
$$

where $j_{M}(Z)=\operatorname{det}(C Z+D)$. In many instances it will be clear that we work with modular forms of weight $k$, so in these cases we omit the $k$ and write simply $(F \mid[M])(Z)$. Furthermore, we set

$$
\Gamma_{0}^{(n)}(N)=\left\{M \in \operatorname{Sp}_{2 n}(\mathbb{Z}) \mid M=\left(\begin{array}{ll}
A & B \\
C & D
\end{array}\right) \text { with } C \equiv 0(\bmod N)\right\}
$$

and let $M_{k}^{(n)}(N)$ denote the vector space of all functions $F: \mathbb{H}_{n} \rightarrow \mathbb{C}$ that satisfy $F \mid[M]=F$ for all $M \in \Gamma_{0}^{(n)}(N)$. To hold notation simple, we omit the degree in the superscript if it is clear from the context. For $n=1$, we impose the additional condition that $F$ is regular at all cusps, which holds automatically by Koecher's principle for $n>1$. Any $F \in M_{k}^{(n)}(N)$ has a Fourier expansion

$$
F(Z)=\sum_{T \in \mathcal{S}} A(T) e(\operatorname{tr}(T Z)),
$$

where $\mathcal{S}_{n}$ denotes all $n \times n$ positive semi-definite, half-integer matrices with integer entries on the diagonal. These Fourier coefficients satisfy $A(T)=A\left(U^{T} T U\right)$ for $U \in \mathrm{GL}_{2}(\mathbb{Z})$. Recall that a positive matrix $A$ with diagonal entries $a_{1}, \ldots, a_{n}$ is called Minkowski-reduced, if $0<a_{1} \leqslant \ldots \leqslant a_{n}$ and all off-diagonal entries satisfy $\left|a_{i l}\right| \leqslant \min \left(a_{i}, a_{l}\right)$. In the following, we denote the set of Minkowski-reduced matrices that are contained in $\mathcal{S}_{n}$ by $\mathcal{R}_{n}$. In every class, there is at least one $T \in \mathcal{R}_{n}$, so when evaluating $A(T)$ it is often handy to assume that $T \in \mathcal{R}_{n}$.

Moreover, we define the Siegel- $\Phi$-operator by

$$
\Phi^{(r)}(F)(Z)=\lim _{t \rightarrow \infty} F\left(\begin{array}{ll}
Z & \\
& i t I_{r}
\end{array}\right)
$$

that maps $M_{k}^{(n)}(N) \rightarrow M_{k}^{(n-r)}(N)$. For simplicity, we write $\Phi^{(r)}(F)(Z)$ for $r=1$.

In the following we focus on the case that $n=2$. We denote by $\mathrm{S}(N)$ the Satake compactification of $\Gamma_{0}^{(2)}(N) \backslash \mathbb{H}_{2}$ and by $\operatorname{Bd}(N)$ the boundary of $\mathrm{S}(N)$. Satake [48] proved that the boundary components of $\operatorname{Bd}(N)$ are modular curves and that they intersect at various cusps of these curves. We call the one- and zero-dimensional components of $\operatorname{Bd}(N)$ 1-cusps and 0-cusps respectively.

The subspace of cusp forms in $M_{k}^{(2)}(N)$ is given by those forms that vanish at $\operatorname{Bd}(N)$. To introduce this formally, we set $\Phi_{K}(F)(z)=\Phi^{(1)}(F \mid[K])(z)$ and define

$$
S_{k}^{(2)}(N)=\left\{F \in M_{k}^{(2)}(N) \mid \Phi_{K}(F)=0 \text { for all } K \in \operatorname{Sp}_{4}(\mathbb{Z})\right\}
$$


The 1-cusps correspond bijectively to double cosets of $\Gamma_{0}^{(2)}(N) \backslash \operatorname{Sp}_{4}(\mathbb{Q}) / P(\mathbb{Q})$ where $P(\mathbb{Q})$ is the maximal parabolic subgroup of $\operatorname{Sp}_{4}(\mathbb{Q})$ defined by

$$
P(\mathbb{Q})=\left\{\left(\begin{array}{llll}
\mathbb{Q} & 0 & \mathbb{Q} & \mathbb{Q} \\
\mathbb{Q} & \mathbb{Q} & \mathbb{Q} & \mathbb{Q} \\
\mathbb{Q} & 0 & \mathbb{Q} & \mathbb{Q} \\
0 & 0 & 0 & \mathbb{Q}
\end{array}\right)\right\} \cap \operatorname{Sp}_{4}(\mathbb{Q}) .
$$

To show that $F$ is a cusp form it suffices to verify that $\Phi_{\gamma_{i}}(F)=0$ for a set of 1-cusp representatives $\gamma_{i} \in \Gamma_{0}^{(2)}(N) \backslash \mathrm{Sp}_{4}(\mathbb{Q}) / P(\mathbb{Q})$. In other words, a form $F$ is cuspidal if the Fourier coefficients at every cusp are only supported on positive definite matrices $T$.

A set of coset representatives of 0 -cusps is given by $\Gamma_{0}^{(2)}(N) \backslash \operatorname{Sp}_{4}(\mathbb{Z}) / \Gamma_{\infty}$, where

$$
\Gamma_{\infty}=\left\{M=\left(\begin{array}{cc}
A & B \\
0 & D
\end{array}\right) \in \operatorname{Sp}_{4}(\mathbb{Z})\right\} .
$$

Explicit sets of representatives for 1- and 0-cusps for squarefree level are given in 9]. This work also specifies at which 0 -cusps the 1-cusp representatives intersect.

As in the case of elliptic modular forms, the space $M_{k}^{(2)}(N)$ can be decomposed into cusp forms and Eisenstein series. We assume that $k$ is even, since there are no Eisenstein series with trivial nebentypus of odd weight. Indeed, $F \in M_{k}^{(3)}(N)$ satisfies $F(Z)=(-1)^{k} F(Z)$, so there are no modular forms of odd weight in $M_{k}^{(3)}(N)$. Besides, the $\Phi$-operator is surjective, implying that all non-cusp forms in $M_{k}^{(2)}(N)$ are contained in the image of $M_{k}^{(3)}(N)$ under the $\Phi$-operator.

\subsubsection{Eisenstein series}

We start by considering the so-called Siegel-Eisenstein series which resemble the classical analogue of degree one:

$$
\mathbb{E}(Z)=\sum_{M \in \Gamma_{\infty} \backslash \Gamma_{0}(N)} j(M, Z)^{-k}
$$

They are obviously well-defined and live in $M_{k}^{(2)}(N)$. Furthermore, they converge absolutely (and uniformly on vertical strips of positive height) for $k \geqslant 4$, cf. [38, p. 67]. The value of $\mathbb{E}(Z)$ at the 0 -cusp represented by $\gamma \in \Gamma_{0}(N) \backslash \operatorname{Sp}_{4}(\mathbb{Z}) / \Gamma_{\infty}$ is given by

$$
\Phi_{\gamma}^{2}(\mathbb{E})=\lim _{t \rightarrow \infty} \mathbb{E} \mid[\gamma](i t I)=\sum_{M \in \Gamma_{\infty} \backslash \Gamma_{0}(N)} \lim _{t \rightarrow \infty} j(M \gamma, i t I)^{-k}
$$

The terms of the latter sum equal 1 if $M \gamma \in \Gamma_{\infty}$ and 0 otherwise, since $M \gamma \in \Gamma_{\infty}$ implies that $\gamma \in \Gamma_{0}(N)$. Hence, $\mathbb{E}(Z)$ takes the value 1 at the cusp $i \infty I_{2}$ and 
vanishes at all other cusps. For each 0 -cusp representative $\gamma_{i}$, we set

$$
\mathbb{E}_{\gamma_{i}}(Z)=\sum_{M \in \Gamma_{\infty} \backslash \gamma_{i}^{-1} \Gamma_{0}(N)} j(M, Z)^{-k}
$$

Then, $\mathbb{E}_{\gamma_{i}}(Z)$ takes the value 1 at the 0 -cusp represented by $\gamma_{i}$ and vanishes at all other 0 -cusps. Indeed, $\gamma_{1}^{-1} \Gamma_{0}(N) \gamma_{2} \subseteq \Gamma_{\infty}$ implies that $\gamma_{1}$ and $\gamma_{2}$ are in the same double coset.

Hence, for any $F \in M_{k}^{(2)}(N)$ we can choose a linear combination of SiegelEisenstein series $\sum c_{i} \mathbb{E}_{\gamma_{i}}$ such that $F-\sum c_{i} \mathbb{E}_{\gamma_{i}}$ vanishes at all 0-cusps; a suitable choice is $c_{i}=\Phi_{\gamma_{i}}^{2}(F)$.

It remains to consider forms that vanish at 0 -cusps but not at all 1 -cusps. For this reason, we need some additional notation. An element $L \in P(\mathbb{Z}):=P(\mathbb{Q}) \cap \operatorname{Sp}_{4}(\mathbb{Z})$ has the form

$$
\left(\begin{array}{cccc}
a_{1} & 0 & b_{1} & b_{2} \\
a_{3} & a_{4} & b_{3} & b_{4} \\
c_{1} & 0 & d_{1} & d_{2} \\
0 & 0 & 0 & d_{4}
\end{array}\right)
$$

with $a_{4}=d_{4}= \pm 1$. We define a map from $P(\mathbb{Z})$ to $\mathrm{SL}_{2}(\mathbb{Z})$ by setting

$$
\omega(L)=\left(\begin{array}{ll}
a_{1} & b_{1} \\
c_{1} & d_{1}
\end{array}\right)
$$

Furthermore, for $Z=\left(\begin{array}{ll}z_{1} & z_{2} \\ z_{2} & z_{4}\end{array}\right) \in \mathbb{H}_{2}$, we set $\pi(Z)=z_{1}$. We set

$$
E(f, Z):=\sum_{M \in P(N) \backslash \Gamma_{0}(N)} f(\pi(M Z)) j(M, Z)^{-k},
$$

where $P(N)=P(\mathbb{Q}) \cap \Gamma_{0}(N)$ and $f \in S_{k}(N)$. Such Eisenstein series were first considered by Klingen [37] and are therefore called Klingen-Eisenstein series. If we write $L \in P(N)$ in the form above we have

$$
\pi(L Z)=\omega(L) z_{1}=\frac{a_{1} z_{1}+b_{1}}{c_{1} z_{1}+d_{1}}
$$

By the cocycle relation

$$
j(L M, Z)=j(L, M Z) j(M, Z)
$$

and $j(L, M Z)=d_{4}\left(c_{1} \pi(M Z)+d_{1}\right)$ we conclude for even $k$ that $f(\pi(L M Z)) j(L M, Z)^{-k}=d_{4}^{-k} f \mid[\omega(L)](\pi(M Z)) j(M, Z)^{-k}=f(\pi(M Z)) j(M, Z)^{-k}$. Thus, $E(f, Z)$ is well defined. Furthermore, $E(f, Z)$ converges for $k>4$ uniformly on vertical strips of positive height, cf. [38, Theorem 1]. The following result was first proved by Klingen [38, Proposition 5]: 
Lemma 27. Let $k>4$. It holds that

$$
\Phi(E(f, \cdot))=f .
$$

Proof. Due to the uniform convergence of the Klingen-Eisenstein series we can interchange limit and sum. As a consequence, we obtain

$$
\Phi\left(E(f, \cdot)(z)=f(z)+\sum_{\substack{M \in P(N) \backslash \Gamma_{0}(N) \\
M \notin P(N)}} \lim _{\lambda \rightarrow \infty} f\left(\pi\left(M\left(\begin{array}{ll}
z & \\
& i \lambda
\end{array}\right)\right)\right) j\left(M,\left(\begin{array}{ll}
z & \\
& i \lambda
\end{array}\right)\right)^{-k} .\right.
$$

It remains to show that each of the terms in latter sum is 0 . Recall that for an elliptic cusp form $f$ it holds that $f(z) \ll(\operatorname{Im} z)^{k / 2}$. Thus, it is sufficient to show for $M \notin P(N)$ that

$$
\lim _{\lambda \rightarrow \infty}\left(\operatorname{Im} \pi\left(M\left(\begin{array}{ll}
z & \\
& i \lambda
\end{array}\right)\right)\right)^{-1} j\left(M,\left(\begin{array}{ll}
z & \\
& i \lambda
\end{array}\right)\right)^{-2}=0 .
$$

Assume that $M=\left(\begin{array}{cc}A & B \\ C & D\end{array}\right)$ and denote the entries of each block matrix by small letters and indices from 1 to 4 , for example $A=\left(\begin{array}{ll}a_{1} & a_{2} \\ a_{3} & a_{4}\end{array}\right)$. Then,

$$
\begin{gathered}
\operatorname{Im} \pi\left(M\left(\begin{array}{ll}
z & \\
& i \lambda
\end{array}\right)\right) j\left(M,\left(\begin{array}{ll}
z & \\
& i \lambda
\end{array}\right)\right)^{2}=\left[\left(a_{1} z+b_{1}\right)\left(c_{4} i \lambda\right)\right. \\
\left.-\left(a_{2} i \lambda+b_{2}\right)\left(c_{3} z+d_{3}\right)\right] \cdot\left[\left(c_{1} \bar{z}+d_{1}\right)\left(-c_{4} i \lambda+d_{4}\right)-\left(-c_{2} i \lambda+d_{2}\right)\left(c_{3} z+d_{3}\right)\right] .
\end{gathered}
$$

We interpret the result as a polynomial in $\lambda$. The $\lambda^{2}$ coefficient is given by

$$
\left(a_{1} c_{4}^{2} d_{1}-b_{1} c_{1} c_{4}^{2}-a_{2} c_{3} c_{4} d_{1}+a_{2} c_{1} c_{4} d_{3}-a_{1} c_{2} c_{4} d_{3}+b_{1} c_{2} c_{3} c_{4}\right) y .
$$

Since $M \in \operatorname{Sp}_{4}(\mathbb{Z})$ we have that $A^{T} D-C^{T} B=I$ and $A^{T} C$ is symmetric. This gives us the following three relations:

$$
\begin{aligned}
& a_{1} d_{1}+a_{3} d_{3}-b_{1} c_{1}-b_{3} c_{3}=1 \\
& a_{2} d_{1}+a_{4} d_{3}-b_{1} c_{2}-b_{3} c_{4}=0 \\
& a_{1} c_{2}+a_{3} c_{4}-a_{2} c_{1}-a_{4} c_{3}=0
\end{aligned}
$$

Solving the second term for $b_{3}$ and the third for $a_{3}$ and plugging this into the first equation, we conclude

$$
a_{1} d_{1}-b_{1} c_{1}-\frac{a_{2} c_{3} d_{1}}{c_{4}}+\frac{a_{2} c_{1} d_{3}}{c_{4}}-\frac{a_{1} c_{2} d_{3}}{c_{4}}-\frac{b_{1} c_{2} c_{3}}{c_{4}}=1
$$


Thus, the $\lambda^{2}$ coefficients is $c_{4}^{2}$. The other terms can be computed similarly and display $(74)$ equals

$$
c_{4}^{2} y \lambda^{2}+\left|c_{3} z+d_{3}\right|^{2} \lambda+d_{4}^{2} y
$$

Hence, (73) is satisfied unless, $c_{3}=c_{4}=d_{3}=0$. This yields the claim since $M \in P(N)$ if and only if $M \in \operatorname{Sp}_{4}(\mathbb{Z})$ and $\lambda(M)=(0,0,0, *)$, where $\lambda(M)$ denotes the bottom row of matrix.

In order to obtain a basis of $M_{k}^{(2)}(N)$, we define a Klingen-Eisenstein series for each 1-cusp representative. We assume that the level is squarefree as this allows us to consider a more easily manageable set of 1-cusp representatives. For this purpose, it is handy to identify $\Gamma_{0}^{(2)}(N) \backslash \mathrm{Sp}_{4}(\mathbb{Q}) / P(\mathbb{Q})$ with $\Gamma_{0}^{(2)}(N) \backslash \mathrm{GSp}_{4}(\mathbb{Q}) / P^{*}(\mathbb{Q})$, where $P^{*}(\mathbb{Q})$ is the maximal parabolic subgroup in $\mathrm{GSp}_{2}(\mathbb{Q})$ that contains $P(\mathbb{Q})$. The main advantage of latter coset consists in the possibility to choose representatives $\kappa_{i}$ such that $\kappa_{1}^{-1} \Gamma_{0}(N) \kappa_{i}=\Gamma_{0}(N)$. For each divisor $l$ of the (squarefree) level $N$, we fix a matrix

$$
\operatorname{Sp}_{4}(\mathbb{Z}) \ni \gamma(l) \equiv \begin{cases}\left(\begin{array}{ll} 
& -I \\
I &
\end{array}\right) & \bmod l^{2} \\
\left(\begin{array}{ll}
I & \\
& I
\end{array}\right) & \bmod (N / l)^{2}\end{cases}
$$

and set

$$
\kappa(l):=\gamma(l)\left(\begin{array}{ll}
l I & \\
& I
\end{array}\right) .
$$

Then, $\kappa(l)$ for $l \mid N$ is a set of representatives of $\Gamma_{0}(N) \backslash \mathrm{GSp}_{2}(\mathbb{Q}) / P^{*}(\mathbb{Q})$, cf. [9], Section 2.2.] or [16, Section 7]. We set

$$
E_{l}(f, Z)=E(f, Z) \mid\left[\kappa_{l}\right] \text { and } \Phi_{l}(F)=\Phi\left(F \mid\left[\kappa(l)^{-1}\right]\right) .
$$

Corollary 28. Let $m, l$ denote divisors of $N$. It holds that

$$
\Phi_{m}\left(E_{l}(f, Z)= \begin{cases}f & \text { if } l=m, \\ 0 & \text { otherwise } .\end{cases}\right.
$$

Proof. By definition, we have

$$
\Phi_{l}\left(E_{l}(f, \cdot)\right)=\Phi(E(f, \cdot))=f .
$$

The argument in Lemma 27 also works when we consider $\operatorname{GSp}_{4}(\mathbb{Q})$ and $P^{*}(\mathbb{Q})$ instead of $\Gamma_{0}(N)$ and $P(N)$. Thus, we have for $Z_{\lambda}=\left(\begin{array}{ll}z & \\ & i \lambda\end{array}\right)$ that

$$
\lim _{\lambda \rightarrow \infty} f\left(\pi\left(M Z_{\lambda}\right)\right) j\left(M, Z_{\lambda}\right)^{-k}=0 \text { for } M \in \mathrm{GSp}_{4}(\mathbb{Q}) \backslash P^{*}(\mathbb{Q}) .
$$


Hence, if there is no element $M \in \Gamma_{0}^{(2)}(N)$ such that $M \kappa(l) \kappa(m)^{-1} \in P^{*}(\mathbb{Q})$ for $m \neq l$ it follows that $\Phi_{m}\left(E_{l}(f, Z)=0\right.$. Let's assume that there is such an $M \in$ $\Gamma_{0}^{(2)}(N)$. This implies that $\lambda\left(M \kappa(l) \kappa(m)^{-1}\right)=(0,0,0, *)$. By construction, all entries of

$$
m M \kappa(l) \kappa(m)^{-1}=M \gamma(l)\left(\begin{array}{ll}
l I & \\
& m I
\end{array}\right) \gamma(m) \in \operatorname{GSp}_{4}(\mathbb{Q})
$$

are integers. Assume there is $t>1$ such that $t \mid l$ and $t \nmid m$. For $\lambda(M)=$ $\left(c_{3}, c_{4}, d_{3}, d_{4}\right)$ we have

$$
\lambda\left(m M \kappa(l) \kappa(m)^{-1}\right) \equiv \lambda\left(M\left(l^{-m I}\right)\right) \equiv\left(l d_{3}, l d_{4},-m c_{3},-m c_{4}\right) \quad\left(\bmod t^{2}\right) .
$$

Consequently, we that $d_{3}, d_{4} \equiv 0(\bmod t)$ as $l$ is squarefree. As $t \mid N$, every entry in the lower row of $M$ is divisible by $t$. However, this contradicts the assumption that $M \in \Gamma_{0}^{(2)}(N)$.

For $t \nmid l$ and $t \mid m$ we obtain

$$
\lambda\left(m M \kappa(l) \kappa(m)^{-1}\right) \equiv\left(m d_{3}, m d_{4},-l c_{3},-l c_{4}\right) \quad\left(\bmod t^{2}\right)
$$

and the very same contradiction.

Consider $F \in M_{k}^{(2)}(N)$ for squarefree $N$ and set $f_{l}=\Phi_{l}(F)$ and $c_{i}=\Phi_{\gamma_{i}}^{2}(F)$ for a set of representatives $\gamma_{i}$ of 0 -cusps. Then

$$
F(Z)-\sum_{l \mid N} E\left(f_{l}, Z\right)-\sum_{i} c_{i} \mathbb{E}_{\gamma_{i}}(Z) \in S_{k}^{(2)}(N)
$$

Hence, we get the following decomposition:

Corollary 29. For squarefree $N$ we have

$$
\begin{aligned}
M_{k}^{(2)}(N)=S_{k}^{(2)}(N) & \oplus\left\langle E_{l}(f, Z)\left|f \in S_{k}^{(1)}(N), l\right| N\right\rangle \\
& \oplus\left\langle\mathbb{E}_{\gamma}(Z) \mid \gamma \in \Gamma_{0}(N) \backslash \operatorname{Sp}_{4}(\mathbb{Z}) / \Gamma_{\infty}\right\rangle .
\end{aligned}
$$

For arbitrary level, a set of 1-cusp representatives and the corresponding decomposition of the Klingen-Eisenstein space can be found in [54, Corollary 3.3 and $3.4]$.

\subsubsection{Theta series and representation of quadratic forms}

As the theta series satisfies $\theta(Q, Z)=\theta\left(U^{T} Q U, Z\right)$ for $U \in \mathrm{GL}_{m}(\mathbb{Z})$ it only depends on the class of $Q$. As in the case of elliptic modular forms we approximate the 
theta series by a weighted average over the classes in the genus. Recall that $o(Q)=\#\left\{U \in \mathrm{GL}_{2 m}(\mathbb{Z}) \mid Q=U^{T} Q U\right\}$ is finite. We set

$$
\theta(\operatorname{gen} Q, Z)=\left(\sum_{R \in \operatorname{gen}(Q)} \frac{1}{o(R)}\right)^{-1} \sum_{R \in \operatorname{gen}(Q)} \frac{\theta_{R}(Z)}{o(R)}=\sum_{T \in \mathcal{S}} r(\operatorname{gen} Q, T) e(\operatorname{tr} T Z) .
$$

The Fourier coefficients were explicitly computed by Siegel:

$$
r(\operatorname{gen} Q, T)=\frac{\pi^{2 k-\frac{1}{2}}(\operatorname{det} T)^{k-\frac{3}{2}}}{\Gamma(k) \Gamma(k-1) \operatorname{det} Q} \prod_{p} \beta_{p}(Q, T)
$$

where the $p$-adic densities are given by

$$
\beta_{p}(Q, T)=\lim _{t \rightarrow \infty} p^{t(3-4 k)} \#\left\{G \in M_{m, n}\left(\mathbb{Z} / p^{t} \mathbb{Z}\right) \mid G^{T} Q G \equiv T\left(\bmod p^{t}\right)\right\} .
$$

Our first aim is to show that $\theta(Q, Z)-\theta\left(Q^{\prime}, Z\right)$ vanishes at all 0-cusps if $Q$ and $Q^{\prime}$ are in the same genus. By the results above, this enables us to express the difference $\theta(Q, Z)-\theta\left(Q^{\prime}, Z\right)$ as a linear combination of Klingen-Eisenstein series and cusp forms.

To this end, we introduce the generalized theta series for $A, B \in M_{2,2 k}(\mathbb{C})$

$$
\theta_{(A, B)}(Q, Z)=\sum_{X \in M_{2 k, 2}(\mathbb{Z})} e\left(\frac{1}{2} \operatorname{tr}\left((X+A)^{T} Q(X+A)+2 B^{T} X\right)\right)
$$

that satisfies the transformation formula

$$
\theta_{(A, B)}\left(Q^{-1}, Z^{-1}\right)=e\left(-\operatorname{tr}\left(A^{T} B\right)\right) \operatorname{det} Q(\operatorname{det} Z / i)^{k} \theta_{(B,-A)}(Q, Z),
$$

cf. [19, Satz 0.13]. Note that $\theta_{(A, B)}(Q, Z)=\theta_{(-A,-B)}(Q, Z)$.

Before we compute the value at each 0-cusp, we need to determine a set of suitable representatives:

Lemma 30. In every coset class of $\Gamma_{0}(N) \backslash \mathrm{Sp}_{4}(\mathbb{Z}) / \Gamma_{\infty}$ there is $M=\left(\begin{array}{ll}A & B \\ C & D\end{array}\right)$ with $\operatorname{det} C \neq 0$.

Proof. Consider $M \in \mathrm{Sp}_{4}(\mathbb{Z})$ with $\operatorname{det} C=0$. By left and right multiplication with $\Gamma_{\infty}$, we can assume that

$$
C=\left(\begin{array}{cc}
c_{1} & 0 \\
0 & 0
\end{array}\right)
$$

If $c_{1}=0$ then $\operatorname{det} A \neq 0$ and left multiplication by

$$
K=\left(\begin{array}{cc}
* & * \\
\tilde{C} & \tilde{D}
\end{array}\right) \in \Gamma_{0}(N)
$$


with $\operatorname{det} \tilde{C} \neq 0$ gives a matrix with the desired properties. For $c_{1} \neq 0$ we still have $a_{2} \neq 0$ or $a_{4} \neq 0$ by $A^{T} D-B^{T} C=I$. In the former case we choose $\tilde{C}=\left(\begin{array}{cc}0 & 0 \\ \tilde{c}_{3} & 0\end{array}\right)$ with $\tilde{c}_{3} \neq 0$ and $\tilde{D}=\left(\begin{array}{cc}\tilde{d}_{1} & * \\ \tilde{d}_{3} & *\end{array}\right)$ with $\tilde{d}_{1} \neq 0$. Then,

$$
\operatorname{det}(\tilde{C} A+\tilde{D} C)=\operatorname{det}\left(\begin{array}{cc}
\tilde{d}_{1} c_{1} & 0 \\
\tilde{c}_{3} a_{1}+\tilde{d}_{3} c_{1} & \tilde{c}_{3} a_{2}
\end{array}\right)=\tilde{d}_{1} c_{1} \tilde{c}_{3} a_{2} \neq 0 \text {. }
$$

The latter case works analogously, we choose $\tilde{C}=\left(\begin{array}{cc}0 & 0 \\ 0 & \tilde{c}_{4}\end{array}\right)$ and $\tilde{D}$ as before. This yields

$$
\operatorname{det} \tilde{C} A+\tilde{D} C=\tilde{d}_{1} c_{1} \tilde{c}_{4} a_{4} \neq 0
$$

For a representative with $\operatorname{det} C \neq 0$, we $\operatorname{decompose} M$ into

$$
\left(\begin{array}{ll}
A & B \\
C & D
\end{array}\right)=\left(\begin{array}{cc}
I & A C^{-1} \\
& I
\end{array}\right)\left(\begin{array}{ll} 
& -C^{-T} \\
C &
\end{array}\right)\left(\begin{array}{cc}
I & C^{-1} D \\
& I
\end{array}\right)
$$

This decomposition allows us to get a similar transformation formula for the action of a 0 -cusp representative on the theta series as in the case $n=1$ :

Lemma 31. Consider the decomposition of $M$ with $\operatorname{det} C \neq 0$ as above. Then

$$
\theta(Q, Z) \mid[M]=\sum_{X \in M_{2 k, 2}(\mathbb{Z})} \alpha(X, Q, M) e\left(\frac{1}{2} X^{T} Q^{-1} X Z\right)
$$

with

$$
\begin{aligned}
\alpha(X, Q, M) & =\frac{\omega}{(\operatorname{det} C)^{k} \operatorname{det} Q} e\left(\frac{1}{2} \operatorname{tr}\left(X^{T} Q^{-1} X C^{-1} D\right)\right) \\
& \times \sum_{X_{1}^{T} \in M_{2,2 k}(\mathbb{Z}) / C M_{2,2 k}(\mathbb{Z})} e\left(\frac{1}{2} \operatorname{tr}\left(2 C^{-1} X_{1}^{T} X+A C^{-1} X_{1}^{T} Q X_{1}\right)\right),
\end{aligned}
$$

where $\omega$ is either 1 or -1 .

Proof.

$$
\begin{aligned}
\theta(Q, Z) \mid & {\left[\left(\begin{array}{cc}
I & A C^{-1} \\
I
\end{array}\right)\left(\begin{array}{ll}
C^{-T} & -C^{-T}
\end{array}\right)\right] } \\
& =(\operatorname{det} C Z)^{-k} \sum_{X \in M_{2 k, 2}(\mathbb{Z})} e\left(\frac{1}{2} \operatorname{tr}\left(X^{T} Q X\left(C^{-T}\left(-Z^{-1}\right) C^{-1}+A C^{-1}\right)\right)\right) .
\end{aligned}
$$


We put $X^{T}=X_{1}^{T}+C X_{2}^{T}$ where $X_{1}^{T}$ runs over matrices in $X_{1}^{T} \in M_{2,2 k}(\mathbb{Z}) / C M_{2,2 k}(\mathbb{Z})$ and $X_{2}$ over matrices in $M_{2 k, 2}(\mathbb{Z})$. Since $C^{T} A C^{-1}=A^{T}$ is integral, the previous display equals

$$
\begin{aligned}
& (\operatorname{det} C Z)^{-k} \sum_{X_{1}^{T} \in M_{2,2 k}(\mathbb{Z}) / C M_{2,2 k}(\mathbb{Z})} e\left(\frac{1}{2} \operatorname{tr}\left(X_{1}^{T} Q X_{1} A C^{-1}\right)\right) \\
& \times \sum_{X_{2} \in M_{2 k, 2}(\mathbb{Z})} e\left(\frac{1}{2} \operatorname{tr}\left(\left(\left(X_{1} C^{-T}\right)^{T}+X_{2}^{T}\right) Q\left(X_{1} C^{-T}+X_{2}\right)(-Z)^{-1}\right)\right) \\
& =(\operatorname{det} C Z)^{-k} \sum_{X_{1}^{T} \in M_{2,2 k}(\mathbb{Z}) / C M_{2,2 k}(\mathbb{Z})} e\left(\frac{1}{2} \operatorname{tr}\left(X_{1}^{T} Q X_{1} A C^{-1}\right)\right) \theta_{\left(X_{1} C^{-T}, 0\right)}\left(Q, Z^{-1}\right) .
\end{aligned}
$$

By applying $(78)$ we have that

$$
\theta_{\left(X_{1} C^{-T}, 0\right)}\left(Q, Z^{-1}\right)=\frac{(-1)^{k}(\operatorname{det} Z)^{k} \theta_{\left(0, X_{1} C^{-T}\right)}\left(Q^{-1}, Z\right)}{\operatorname{det} Q},
$$

where

$$
\theta_{\left(0, X_{1} C^{-T}\right)}\left(Q^{-1}, Z\right)=\sum_{X \in M_{2 k, 2}(\mathbb{Z})} e\left(\frac{1}{2}\left(\operatorname{tr}\left(X^{T} Q^{-1} X Z+2 C^{-1} X_{1}^{T} X\right)\right)\right) .
$$

The claim follows now by taking the third matrix in the decomposition of $M$ into account.

Corollary 32. Let $Q, Q^{\prime}$ be in the same genus. Then $\theta(Q, Z)-\theta\left(Q^{\prime}, Z\right)$ vanishes at all 0-cusps.

Proof. The value of $\theta(Q, Z)$ at the cusp $i \infty I$ is obviously 1 . For all other cusps, we choose a representative with $\operatorname{det} C \neq 0$ and apply Lemma 31. Consequently, the value of $\theta(Q, Z)$ at the cusp represented by $M^{-1}$ is given by

$$
\frac{\omega}{(\operatorname{det} C)^{k} \operatorname{det} Q} \sum_{X_{1}^{T} \in M_{2,2 k}(\mathbb{Z}) / C M_{2,2 k}(\mathbb{Z})} e\left(\frac{1}{2} \operatorname{tr}\left(X_{1}^{T} Q X_{1} A C^{-1}\right)\right) .
$$

This term only depends on the discriminant and the congruence class of the underlying quadratic form, thus, it is genus invariant.

By Corollary 29 and Corollary 32 we obtain the following decomposition for squarefree level:

$$
\theta(Q, Z)=\theta(\operatorname{gen} Q, Z)+\sum_{l \mid N} E_{l}\left(f_{l}, Z\right)+G(Z)
$$


where $G(Z)$ is a cusp form of degree two and $f_{l}(z)$ are cusp forms of degree one. To determine $f_{l}$ we apply the $\Phi_{l}$ operator which yields

$$
f_{l}(z)=\lim _{t \rightarrow \infty}\left(\left(\theta(Q, *) \mid\left[\kappa(l)^{-1}\right]\right)\left(\begin{array}{ll}
z & \\
& i t
\end{array}\right)-\left(\theta(\operatorname{gen} Q, *) \mid\left[\kappa(l)^{-1}\right]\right)\left(\begin{array}{ll}
z & \\
& i t
\end{array}\right)\right) .
$$

On the level of Fourier coefficients, this corresponds to

$$
r(Q, T)=r(\operatorname{gen} Q, T)+\sum_{l \mid N} A_{l}(T)+S(T),
$$

where $A_{l}(T), g(T)$ are the Fourier coefficients of $E_{l}\left(f_{l}, Z\right)$ and $G(Z)$. For $T>0$, the followings bounds for $A_{l}(T)$ and $g(T)$ are known by Kitaoka, cf. [30] and [31,

$$
A_{l}(T) \ll_{N, k}(\operatorname{det} T)^{k-\frac{3}{2}} \min (T)^{\frac{1-k}{2}} \quad g(T) \ll_{N, k}(\operatorname{det} T)^{\frac{k}{2}-\frac{1}{4}} .
$$

Furthermore, Kitaoka [29], [32] has shown that the product over the $p$-adic densities for $2 k \geqslant 7$ is bounded from below by a constant depending only on $Q$ as long as $T$ is locally represented by $Q 9^{9}$ By means of $(76)$ this gives a lower bound for $r($ gen $Q, T)$ and hence, an asymptotic formula for $r(Q, T)$ with respect to $T$.

The goal of this thesis is to make these estimates uniform in the level $N$. The treatment of the main term is rather straightforward, since we can apply a formula from Yang [63] to evaluate the local densities at odd primes. The evaluation of the error term, however, is very elaborate. To estimate the Fourier coefficients of the Klingen-Eisenstein series we modify Kitaoka's work [34. The main challenge lies in bounding the Fourier coefficients of the cusps form $G(Z)$. To do so, one expresses the cusp form as a linear combination of Poincaré series and then applies the Kitaoka-Petersson formula to evaluate the Fourier coefficients of the latter. This way, Chida, Katsurada and Matsumoto [15] obtained the following bound:

$$
g(T) \ll \sqrt{\langle G, G\rangle}\left((\operatorname{det} T)^{\frac{k}{2}-\frac{1}{4}+\epsilon} N^{-\frac{1}{2}+\epsilon}+1\right) .
$$

To compute the inner product we make use of the fundamental domain of the full modular group $\operatorname{Sp}_{4}(\mathbb{Z})$. This implies that we need to bound the Fourier coefficients of $G \mid[M]$ where $M$ runs over a set of representatives in $\Gamma_{0}^{(2)}(N) \backslash \operatorname{Sp}_{4}(\mathbb{Z})$.

For the treatment of the error term, we assume for simplicity that the level $N$ is prime. However, an extension of the proof to squarefree level is sketched out in Section 4.4.

\subsection{The main term}

The dominant term in the asymptotic formula for $r(Q, T)$ is given by $r$ (gen $Q, T)$. By (76), we only need to treat the local densities $\beta(p, Q)$ given in (77) to obtain

\footnotetext{
${ }^{9}$ More precisely, Kitaoka shows that if $T$ is locally represented by $Q$, then, for every $p$ and some fixed $a$ there is a solution of $X^{T} Q X \equiv T\left(\bmod p^{a}\right)$ that can be lifted to $p^{(4 k-3) t}$ solutions modulo $p^{a+t}$.
} 
a lower bound for $r$ (gen $Q, T$ ). To this end, we apply Yang's explicit formula for local densities, cf. [63, Theorem 7.1]. Due to the complexity of the formula we only use it in some specific cases and only for odd $p$. Recall that counting solutions of $\frac{1}{2} X^{T} Q X=T$ with $X \in M_{2 k, 2}(\mathbb{Z})$ corresponds to counting solutions of $x, y \in \mathbb{Z}^{2 k}$ of

$$
q(x)=\frac{1}{2} x^{T} Q x=t_{1}, \quad q(x, y):=x^{T} Q y=t_{2}, \quad q(y)=\frac{1}{2} y^{T} Q y=t_{4} .
$$

Lemma 33. Assume that $N$ is odd and squarefree, that $T$ is locally represented by $Q$ and that for all primes $p \mid N$ either $(\operatorname{det} T, p)=1$ or $p^{2 k-4} \nmid \operatorname{det} Q$. Then,

$$
\prod_{p \neq 2} \beta_{p}(Q, T) \gg N^{-\epsilon}
$$

Proof. For $p \nmid 2 \operatorname{det} T \operatorname{det} Q$, the $p$-adic densities are computed explicitly by Siegel [55] and we have that $\beta_{p}(Q, Z) \geqslant 1-p^{1-k}$. If $p \nmid 2 \operatorname{det} T$, we apply [63, Theorem 7.1]. For this purpose, we use that $T$ is equivalent over $\mathbb{Z}_{p}$ to $\operatorname{diag}\left(\alpha_{1} p^{a}, \alpha_{2} p^{b}\right)$ for units $\alpha_{1}, \alpha_{2}$ and $q$ to $q_{0}+p q_{1}$ where $q_{0}, q_{1}$ are diagonal quadratic forms of unit discriminant. Let $v(l)$ be defined as in 60 and set $\chi_{p}(\alpha):=\left(\frac{\alpha}{p}\right)$. If $a=b=0$, the formula yields for even $\operatorname{dim} q_{0}$ that

$$
\beta_{p}(Q, T)=1+v(1) \chi_{p}\left(-\alpha_{1} \alpha_{2}\right) p^{1-\frac{\operatorname{dim} q_{0}}{2}}-\left(v(1)+\chi_{p}\left(-\alpha_{1} \alpha_{2}\right)\right) p^{-\frac{\operatorname{dim} q_{0}}{2}}
$$

and for odd $\operatorname{dim} q_{0}$ that

$$
\beta_{p}(Q, T)=1-p^{1-\operatorname{dim} q_{0}} .
$$

If $\operatorname{dim} q_{0}=1$, there is obviously no solution. For $\operatorname{dim} q_{0}=2$, we get that

$$
\beta_{p}(Q, T)=1+v(1) \chi_{p}\left(-\alpha_{1} \alpha_{2}\right)-p^{-1}\left(v(1)+\chi_{p}\left(-\alpha_{1} \alpha_{2}\right)\right) .
$$

If $v(1) \chi_{p}\left(-\alpha_{1} \alpha_{2}\right)=-1$, then $v(1)=-\chi_{p}\left(-\alpha_{1} \alpha_{2}\right)$ which contradicts $\beta_{p}(Q, T)>0$. Hence, $\beta_{p}(Q, T) \geqslant 2-\frac{2}{p}$. For $\operatorname{dim} q_{0} \geqslant 3$ we see directly that $\beta_{p}(Q, T) \geqslant 1-\frac{1}{p}$.

For arbitrary $T$ and odd $p$, the terms in Yang's formula get very small as long as $\operatorname{dim} Q_{0} \geqslant 5$, since then $d(l) \leqslant-\frac{3}{2} l$, cf. 60). If we trivially estimate the terms $I_{1, i}, I_{2, j}$ with $1 \leqslant i \leqslant 4,1 \leqslant j \leqslant 8$ in the corrected version of [63, p. 25-26], we obtain the lower bound

$$
\beta_{p}(Q, T) \geqslant 1-\frac{2}{p}-\frac{2}{p^{2}} .
$$

Since $p \geqslant 3$, the claim follows.

It remains to treat the case of $p=2$. Therefore, recall that $q$ is equivalent over $\mathbb{Z}_{2}$ to a form

$$
q_{0}+2 q_{1}+2^{2} q_{2}+\ldots
$$

where $q_{i}$ consist of diagonal terms of the form $u_{i} x_{i}^{2}$ with units $u_{i}$ and the binary quadratic forms $x_{1}^{2}+x_{1} x_{2}+x_{2}^{2}$ and $x_{1} x_{2}$. If $\operatorname{dim} q_{0}$ is sufficiently large, we can compute the 2 -adic densities as follows: 
Lemma 34. Let $q=\frac{1}{2} x^{T} Q x$ (with $Q>0$ and integral) be equivalent over $\mathbb{Z}_{2}$ to a form $q_{0}+q_{1}$, where

$$
q_{0}=\sum_{j=1}^{r}\left(x_{2 j-1}^{2}+x_{2 j-1} x_{2 j}+x_{2 j}^{2}\right)+\sum_{l=r+1}^{r+s} x_{2 l-1} x_{2 l}+\sum_{i=2 s+2 r+1}^{6} u_{i} x_{i}^{2} .
$$

with $r+s \leqslant 3$ and $u_{i} \in \mathbb{Z}_{2}^{\times}$, and $q_{1}$ is any form in $m-6$ variables. Then, $\beta_{2}(Q, T) \gg 1$ for any choice of $T$.

Proof. Note that there also exists a formula from Yang [64] for $p=2$, but due to its complexity, we refrain from applying it. Instead, we enumerate all possibilities modulo 8 of quadratic forms $g_{i}$ in six variables as in display (81). By using a suitable computer program such as Python, we can show that for for any combination $t_{1}, t_{2}, t_{4}(\bmod 8)$ there is a solution such that

$$
g_{i}(x) \equiv t_{1}, \quad g_{i}(x, y) \equiv t_{2}, \quad g_{i}(y) \equiv t_{4}(\bmod 8)
$$

where $x_{1}, y_{2}$ are odd, $y_{1}$ even and $x_{1}, x_{2}$ belong to a block that either consists of two diagonal forms or one binary form. By choosing the remaining variables arbitrarily, it follows that for any combination $\left(t_{1}, t_{2}, t_{4}\right)$ there are at least $2^{3(m-6)}$ solutions of $q(x) \equiv t_{1}, q(x, y) \equiv t_{2}, q(x, y) \equiv t_{4}(\bmod 8)$ such that $x_{1}, y_{2}$ are odd and $y_{1}$ is even. If we can show that each of these solution can be lifted to modulus $2^{a}$ with multiplicity $2^{(a-3)(m-3)}$, the claim of the lemma follows.

First, we treat the case that the first block of $q_{0}$ consists of diagonal terms. Assume there is a solution

$$
\begin{aligned}
u_{1} x_{1}^{2}+u_{2} x_{2}^{2}+g\left(x_{3}, \ldots, x_{m}\right) & \equiv t_{1}\left(\bmod 2^{a+1}\right) \\
2 u_{1} x_{1} y_{1}+2 u_{2} x_{2} y_{2}+g\left(x_{3}, y_{3}, \ldots, x_{m}, y_{m}\right) & \equiv t_{2}\left(\bmod 2^{a+1}\right) \\
u_{1} y_{1}^{2}+u_{2} y_{2}^{2}+g\left(y_{3}, \ldots, y_{m}\right) & \equiv t_{4}\left(\bmod 2^{a+1}\right)
\end{aligned}
$$

such that $x_{1}, y_{2}$ are odd and $y_{1}$ is even. These congruence determine $x_{1}, x_{2}, y_{1}, y_{2}$ only up to modulus $2^{a}$. Fix $x_{3}, \ldots x_{m}, y_{1}, y_{3}, \ldots y_{m}$ modulo $2^{a+1}$. If $\tilde{x}_{1}, \tilde{x}_{2}, \tilde{y}_{2}$ solve the congruence above, then also

$$
\begin{array}{ll}
x_{1}=\tilde{x}_{1}+d_{1} 2^{a} & d_{1} \in\{0,1\}, \\
x_{2}=\tilde{x}_{2}+d_{2} 2^{a} & d_{2} \in\{0,1\}, \\
y_{2}=\tilde{y}_{2}+d_{3} 2^{a} & d_{3} \in\{0,1\} .
\end{array}
$$

Consider any lift of $x_{3}, \ldots x_{m}, y_{1}, y_{3} \ldots, y_{m}$ to modulus $2^{a+2}$. Then, one combination of $x_{1}, x_{2}, y_{2}$ from above already solves the congruences modulo $2^{a+2}$. To see this, we first fix $d_{3}$ such that the third congruence is satisfied. Since $y_{2}$ is even and $y_{1}$ is odd, we can choose $d_{1}, d_{2}$ such that

$$
\begin{aligned}
u_{1} x_{1}^{2}+u_{2} x_{2}^{2}+d_{1} 2^{a+1}+d_{2} x_{2} 2^{a+1} & \equiv t_{1}-g\left(x_{3}, \ldots x_{m}\right) & & \left(\bmod 2^{a+2}\right) \\
2 u_{1} x_{1} y_{1}+2 u_{2} x_{2} y_{2}+d_{2} 2^{a+1} & \equiv t_{2}-g\left(x_{3}, y_{3}, \ldots x_{m}, y_{m}\right) & & \left(\bmod 2^{a+2}\right)
\end{aligned}
$$


is satisfied. The resulting solution $\left(x_{1}, x_{2}, y_{2}\right)$ is only fixed modulo $2^{a+1}$ and consequently, gives rise to 8 solutions modulo $2^{a+2}$.

If $x_{1}, x_{2}$ belong to a binary form, the proof is very similar. The congruences

$$
\begin{aligned}
x_{1}^{2}+x_{1} x_{2}+x_{2}^{2} & \equiv t_{1}-g\left(x_{3}, \ldots, x_{m}\right) & & \left(\bmod 2^{a}\right) \\
2 x_{1} y_{1}+x_{1} y_{2}+x_{2} y_{1}+2 x_{2} y_{2} & \equiv t_{2}-g\left(x_{3}, y_{3}, \ldots, x_{m}, y_{m}\right) & & \left(\bmod 2^{a}\right) \\
y_{1}^{2}+y_{1} y_{2}+y_{2}^{2} & \equiv t_{4}-g\left(y_{3}, \ldots, y_{m}\right) & & \left(\bmod 2^{a}\right)
\end{aligned}
$$

already fix $x_{1}, x_{2}, y_{2}$ modulo $2^{a}$. For any lift of $x_{3}, \ldots x_{m}, y_{1}, y_{3}, \ldots y_{m}$, we can choose $d_{1}, d_{2}, d_{3} \in\{0,1\}$ such that

$$
x_{1}+d_{1} 2^{a}, \quad x_{2}+d_{2} 2^{a} \quad y_{2}+d_{3} 2^{a}
$$

satisfy the congruence modulo $2^{a+1}$ since $x_{1}, y_{2}$ are odd and $y_{1}$ is even. The binary quadratic form $x y$ works analogously.

As a consequence, we obtain the following lower bound for the main term:

Corollary 35. Assume that the level $N$ of $Q$ is squarefree. Furthermore, assume that $p^{k-4} \nmid \operatorname{det} Q$ for odd $p$ and $2^{k-5} \nmid \operatorname{det} Q$. Then,

$$
r(\operatorname{gen} Q, T) \gg_{k}(\operatorname{det} T)^{k-\frac{3}{2}}(\operatorname{det} Q)^{-1} N^{-\epsilon} .
$$

\subsection{The error term for prime level}

The aim of this section is to evaluate the error term in the asymptotic formula (79) for $r(Q, T)$. In the following we assume that $k \geqslant 6$ and that the level $N$ is prime. In this case, a set of 1 -cusp representatives in $\Gamma_{0}^{(2)}(N) \backslash \mathrm{GSp}_{4}(\mathbb{Q}) / P^{*}(\mathbb{Q})$ is given by $I_{4}$ and $\eta_{4}=J_{4}\left(\begin{array}{ll}N & \\ & I\end{array}\right), 10$ Thus, the space of Klingen-Eisenstein series is spanned by

$$
\left\langle E\left(f_{1}, Z\right), E_{N}\left(f_{2}, Z\right) \mid f_{1}, f_{2} \in S_{k}(N)\right\rangle
$$

where

$$
E\left(f_{1}, Z\right)=\sum_{M \in P(N) \backslash \Gamma_{0}^{(2)}(N)} j_{M}(Z)^{-k} f_{1}(\pi(M Z))
$$

and

$$
E_{N}\left(f_{2}, Z\right)=E\left(f_{2}, Z\right) \mid\left[\eta_{4}\right]=N^{k} \sum_{M \in P(N) \backslash \Gamma_{0}^{(2)}(N) J_{4}} j(M, N Z)^{-k} f_{2}(\pi(M(N Z))) .
$$

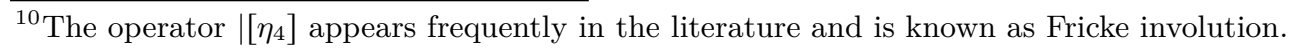


It follows that

$$
\theta(Q, Z)=\theta(\operatorname{gen} Q, Z)+E\left(f_{1}, Z\right)+E_{N}\left(f_{2}, Z\right)+G(Z)
$$

where $G(Z)$ is a cusp form. As previously discussed, the cusp forms $f_{1}, f_{2} \in S_{k}(N)$ can be determined by applying the Siegel $\Phi$-operator. This yields

$$
\begin{aligned}
& f_{1}(z)=\theta(Q, z)-\theta(\operatorname{gen} Q, z) \text { and } \\
& f_{2}(z)=\frac{N^{\frac{k}{2}}}{\sqrt{\operatorname{det} Q}} f_{1} \mid[\eta](z)=\frac{N^{k}}{\operatorname{det} Q}\left(\theta\left(N Q^{-1}, z\right)-\theta\left(\operatorname{gen} N Q^{-1}, z\right)\right) .
\end{aligned}
$$

On the level of Fourier coefficients this corresponds to

$$
r(Q, T)=r(\operatorname{gen} Q, T)+A(T)+B(T)+S(T) .
$$

In the previous section, we already determined a lower bound for $r($ gen $Q, T)$. To bound the Fourier coefficients of the cusp form $G$ we need to evaluate the inner product of $G$. First, we show that for this purpose it suffices to bound the Fourier coefficients of $G \mid[R]$ for $R \in \Gamma_{0}^{(2)}(N) \backslash \mathrm{Sp}_{4}(\mathbb{Z})$. Then, we estimate the absolute value of the Fourier coefficients of the corresponding Klingen-Eisenstein and theta series.

The Klingen-Eisenstein part comprises a lot of work. The underlying idea is to express the Fourier coefficients $A_{R}(T)$ of $E(f, Z) \mid[R]$ as an integral:

$$
A_{R}(T)=\int_{X} \sum_{M \in P(N) \backslash \Gamma_{0}^{(2)}(N) R} f(\pi(M Z)) j(M, Z)^{-k} e\left(-\frac{\operatorname{tr}(T X)}{N}\right)
$$

where $Z=X+i Y$ and $X=\left(\begin{array}{ll}x_{1} & x_{2} \\ x_{2} & x_{4}\end{array}\right)$ runs over $x_{1}, x_{2}, x_{4} \in[0, N]$. To compute this integral, we first derive a suitable decomposition of $P(N) \backslash \Gamma_{0}^{(2)}(N) R$ for a set of representatives $R \in \Gamma_{0}^{(2)}(N) \backslash \mathrm{Sp}_{4}(\mathbb{Z})$ in Section 4.3.2. This enables us to obtain explicit formulas for Fourier coefficients of Klingen-Eisenstein series. As a consequence, we can derive upper bounds for $A(T)$ and $B(T)$ and evaluate the contribution of Klingen-Eisenstein series to the inner-product. Finally, we bound the contribution of theta series to the inner product by evaluating symplectic exponential sums.

\subsubsection{Inner product of theta series}

The principal challenge in obtaining an uniform asymptotic formula for the Siegel theta series is to evaluate the inner product of the cuspidal part:

$$
G(Z)=\theta(Q, Z)-\theta(\operatorname{gen}(Q), Z)-E\left(f_{1}, Z\right)-E_{N}\left(f_{2}, Z\right) .
$$


Let $F(N)$ denote a fundamental domain of $\Gamma_{0}^{(2)}(N) \backslash \mathbb{H}_{2}$. To compute

$$
\langle G, G\rangle=\int_{F(N)}|G(Z)|^{2}(\operatorname{det} Y)^{k} \frac{d X d Y}{(\operatorname{det} Y)^{3}}
$$

we proceed as in the one-dimensional case. First, we reduce the computation to the fundamental domain of the full modular group:

$$
\langle G, G\rangle=\left.\sum_{\gamma \in \Gamma_{0}^{(2)}(N) \backslash \operatorname{Sp}_{4}(\mathbb{Z})} \int_{F(1)}|G|[\gamma]_{k}(Z)\right|^{2}(\operatorname{det} Y)^{k} \frac{d X d Y}{(\operatorname{det} Y)^{3}} .
$$

The usual choice for $F:=F(1)=\operatorname{Sp}_{4}(\mathbb{Z}) \backslash \mathbb{H}_{2}$ is the set of Siegel reduced points. A point $Z=X+i Y$ is called Siegel reduced if $\left|\operatorname{det} j_{M}(Z)\right| \geqslant 1$ for all $M \in \operatorname{Sp}_{4}(\mathbb{Z})$, if $Y$ is Minkowski-reduced and $X$ is reduced modulo 1. As first shown by Gottschling [22] this fundamental domain is explicitly determined by 13 equations. For our computations, we require that

$$
y_{1} \geqslant \frac{\sqrt{3}}{2} \text { and }\left|y_{2}\right| \leqslant \frac{y_{1}}{2} \leqslant \frac{y_{4}}{2} .
$$

The first step in computing (84) is to find a suitable set of right cosets for $\Gamma_{0}^{(2)}(N) \backslash \mathrm{Sp}_{4}(\mathbb{Z})$. If $N$ is prime, this is fairly easy:

Lemma 36. A set of right coset representatives of $\Gamma_{0}^{(2)}(N) \backslash \operatorname{Sp}_{4}(\mathbb{Z})$ for $N$ prime is given by

$$
\begin{aligned}
& R(0)=I_{4}, \\
& R(1)=\iota_{1}(J)\left\{\iota_{1}\left(\left(\begin{array}{ll}
1 & \alpha \\
& 1
\end{array}\right)\right) \mid 0 \leqslant \alpha \leqslant N-1\right\}, \\
& R(2)=\iota_{2}(J)\left\{\left(\begin{array}{ccc}
1 & & \\
\beta_{1} & 1 & \beta_{2} \\
& 1 & -\beta_{1} \\
& & 1
\end{array}\right) \mid 0 \leqslant \beta_{1}, \beta_{2} \leqslant N-1\right\}, \\
& R(3)=J_{4}\left\{\left(\begin{array}{ll}
I & \kappa \\
& I
\end{array}\right) \mid \kappa=\left(\begin{array}{cc}
\kappa_{1} & \kappa_{2} \\
\kappa_{2} & \kappa_{4}
\end{array}\right), 0 \leqslant \kappa_{1}, \kappa_{2}, \kappa_{4} \leqslant N-1\right\} .
\end{aligned}
$$

Proof. See [36, Proposition 2.1].

For more clarity, we recall that

$$
\iota_{1}(J)=\left(\begin{array}{cccc} 
& & -1 & \\
& 1 & & \\
1 & & &
\end{array}\right) \text { and } \iota_{2}(J)=\left(\begin{array}{cccc}
1 & & \\
& & & -1 \\
& & 1 & \\
& & &
\end{array}\right.
$$

We treat the contribution of each coset class to (84) separately. For the simplest case, $R(0)$, we only require an upper bound for the Fourier coefficients of $S$ : 
Lemma 37. Let $G(Z)=\sum_{T \in \mathscr{S}} g(T) e(\operatorname{tr}(T Z))$. Then,

$$
\int_{F}|G(Z)|^{2}(\operatorname{det} Y)^{k-3} d X d Y \ll \sum_{T \in \mathscr{S}}|g(T)|^{2}
$$

where the entries of $T$ run over $t_{1},\left|t_{2}\right|, t_{4} \leqslant C$ for some constant $C$ (that does not depend on $N$ ).

We omit the proof, as it works analogously to the more interesting $R(3)$ case that we consider in the following lemma. For this class, the contribution to $\langle G, G\rangle$ depends on the Fourier coefficients of $G \mid\left[J_{4}\right]$ :

Lemma 38. Let $G \mid\left[J_{4}\right](Z)=\sum_{T \in \mathscr{S}} g_{3}(T) e\left(\frac{\operatorname{tr}(T Z)}{N}\right)$. Then,

$$
\left.\sum_{\gamma \in R(3)} \int_{F}|G|[\gamma]_{k}(Z)\right|^{2}(\operatorname{det} Y)^{k} \frac{d X d Y}{(\operatorname{det} Y)^{3}} \ll N^{2 k} \sum_{\substack{t_{1}, t_{2}, t_{4} \ll N^{1+\epsilon} \\ T>0}} \frac{\left|g_{3}(T)\right|^{2}}{(\operatorname{det} T)^{k-\frac{3}{2}}} .
$$

Proof. The left hand side equals

$$
\begin{aligned}
\int_{\mathrm{SP}_{4}(\mathbb{Z}) \backslash \mathbb{H}_{2}} & \sum_{\kappa \in \mathcal{P}(\mathbb{Z} / N \mathbb{Z})}|(G \mid[J])(Z+\kappa)|^{2}(\operatorname{det} Y)^{k-3} d X d Y \\
=\int_{Y} \sum_{T, T^{\prime} \in \mathscr{S}} g_{3}(T) \overline{g_{3}\left(T^{\prime}\right)} \exp & \left(-\frac{2 \pi \operatorname{tr}\left(T+T^{\prime}\right) Y}{N}\right)(\operatorname{det} Y)^{k-3} d Y \\
& \times \int_{X \bmod N} e\left(\frac{\operatorname{tr}\left(\left(T-T^{\prime}\right) X\right.}{N}\right) d X
\end{aligned}
$$

The $X$ integral vanishes unless $T_{1}=T_{2}$ and equals $N^{3}$ in this case. Taking (85) into account, 86 is bounded by $N^{3} \int_{\sqrt{3} / 2}^{\infty} \int_{-y_{1} / 2}^{y_{1} / 2} \int_{y_{1}}^{\infty} \sum_{T>0}\left|g_{3}(T)\right|^{2} \exp \left(-\frac{4 \pi\left(y_{1} t_{1}+y_{2} t_{2}+y_{4} t_{4}\right)}{N}\right)\left(y_{1} y_{4}-y_{2}^{2}\right)^{k-3} d y_{1} d y_{2} d y_{4}$

where $t_{1}, \frac{t_{2}}{2}$ and $t_{4}$ are the entries of $T$. To compute the integral, we substitute

$$
Y=\left(\begin{array}{ll}
y_{1} & y_{2} \\
y_{2} & y_{4}
\end{array}\right)=\left(\begin{array}{cc}
\gamma_{1} & 0 \\
\gamma_{2} & \gamma_{4}
\end{array}\right)\left(\begin{array}{cc}
\gamma_{1} & \gamma_{2} \\
0 & \gamma_{4}
\end{array}\right)
$$

Note that $y_{4}=\gamma_{2}^{2}+\gamma_{4}^{2}$, so $\gamma_{4}^{2}=y_{4}-\frac{y_{2}^{2}}{y_{1}} \geqslant \frac{3 y_{1}}{4}>\frac{1}{2}$. Hence, the previous display is bounded by $4 N^{3} \sum_{T>0}\left|g_{3}(T)\right|^{2} \int_{\frac{1}{\sqrt{2}}}^{\infty} \int_{-\frac{\gamma_{1}}{2}}^{\frac{\gamma_{1}}{2}} \int_{\frac{1}{\sqrt{2}}}^{\infty} \exp \left(\frac{-4 \pi\left(t_{1} \gamma_{1}^{2}+t_{2} \gamma_{1} \gamma_{2}+t_{4}\left(\gamma_{2}^{2}+\gamma_{4}^{2}\right)\right)}{N}\right) \gamma_{1}^{2 k-4} \gamma_{4}^{2 k-5} d \gamma_{1} d \gamma_{2} d \gamma_{4}$ 
By partial integration, we obtain for the $\gamma_{4}$ integral:

$2 \int_{\frac{1}{\sqrt{2}}}^{\infty} \exp \left(-\frac{4 \pi \gamma_{4}^{2} t_{4}}{N}\right) \gamma_{4}^{2 k-5} d \gamma_{4}=\int_{\frac{1}{2}}^{\infty} \exp \left(-\frac{4 \pi y t_{4}}{N}\right) \gamma_{4}^{k-3} d y \ll\left(\frac{N}{t_{4}}\right)^{k-2} \exp \left(-\frac{t_{4}}{N}\right)$.

Next, we compute the $\gamma_{2}$ integral:

$$
\begin{aligned}
\int_{-\frac{\gamma_{1}}{2}}^{\frac{\gamma_{1}}{2}} \exp \left(-\frac{4 \pi}{N}\left(t_{2} \gamma_{1} \gamma_{2}+t_{4} \gamma_{2}^{2}\right)\right) d \gamma_{2} & =\exp \left(\frac{\gamma_{1}^{2}\left(t_{2}\right)^{2}}{4 t_{4}}\right) \int_{-\frac{\gamma_{1}}{2}}^{\frac{\gamma_{1}}{2}} \exp \left(-\frac{4 \pi t_{4}}{N}\left(\gamma_{2}+\frac{\gamma_{1} t_{2}}{2 t_{4}}\right)^{2}\right) d \gamma_{2} \\
& \leqslant \exp \left(\frac{\gamma_{1}^{2} t_{2}^{2}}{4 t_{4}}\right) \sqrt{\frac{N}{4 \pi t_{4}}} \int_{\frac{\gamma_{1}}{2}\left(t_{2}-t_{4}\right) \sqrt{\frac{4 \pi}{N t_{4}}}}^{\frac{\gamma_{1}}{2}\left(t_{2}+t_{4}\right) \sqrt{\frac{4 \pi}{N t_{4}}}} \exp \left(-\gamma_{2}^{2}\right) d \gamma_{2} .
\end{aligned}
$$

The integral is bounded by $\sqrt{\pi} / 2$. Before applying this, we recall that the complementary Gaussian error function

$$
\operatorname{erfc}(x)=\frac{2}{\sqrt{\pi}} \int_{x}^{\infty} \exp \left(-t^{2}\right) d t \ll x^{-1} \exp \left(-x^{2}\right)
$$

is exponentially decaying in $x$. If $t_{2}>t_{4}\left(\right.$ or $\left.-t_{2}>t_{4}\right)$ the $\gamma_{2}$ integral is bounded by

$$
\exp \left(\frac{\gamma_{1}^{2} t_{2}^{2}}{4 t_{4}}\right) \sqrt{\frac{N}{4 \pi t_{4}}} \operatorname{erfc}\left(\frac{\gamma_{1}\left|t_{2} \mp t_{4}\right| \sqrt{\pi}}{\sqrt{N t_{4}}}\right)
$$

This allows us to truncate the $t_{2}$ sum at

$$
\left|t_{2}\right| \ll t_{4}+\sqrt{t_{4} N} N^{\epsilon}
$$

at the cost of a negligible error. Finally, we estimate the $\gamma_{1}$ integral

$$
\int_{3 / 4}^{\infty} \exp \left(\frac{-4 \pi \gamma_{1}^{2}\left(t_{1}-\frac{t_{2}^{2}}{4 t_{4}}\right)}{N}\right) \gamma_{1}^{2 k-4} d \gamma_{1} \ll\left(\frac{N}{t_{1}-\frac{t_{2}^{2}}{4 t_{4}}}\right)^{k-\frac{3}{2}} \exp \left(-\frac{t_{1}-\frac{t_{2}^{2}}{4 t_{4}}}{N}\right) .
$$

Combining all these computations, 86 is bounded by

$$
N^{3} \sum_{\substack{t_{2} \ll N^{\epsilon}\left(t_{4}+\sqrt{t_{4} N}\right) \\ T \in \mathscr{S}}}\left|g_{3}(T)\right|^{2}\left(\frac{N^{2}}{t_{1} t_{4}-\left(\frac{t_{2}}{2}\right)^{2}}\right)^{k-\frac{3}{2}} \exp \left(-\frac{t_{4}}{N}\right) \exp \left(-\frac{t_{1}-\frac{t_{2}^{2}}{4 t_{4}}}{N}\right) .
$$

The exponential decay allows us to truncate $t_{1}, t_{2}, t_{4}$ at $t_{1}, t_{2}, t_{4} \ll N^{1+\epsilon}$ at the cost of a negligible error. 
The cases $R(1)$ and $R(2)$ are slightly more complex. We start with the observation that

$$
\iota_{1}(-J) \Gamma_{0}(N) \iota_{1}(J) \equiv\left(\begin{array}{cccc}
* & 0 & 0 & * \\
* & * & * & * \\
* & * & * & * \\
* & 0 & 0 & *
\end{array}\right)(\bmod N)
$$

Let $F \in S_{k}(N)$. Then, $F \mid\left[\iota_{1}(J)\right]$ has the following Fourier expansion:

$$
F \mid\left[\iota_{1}(J)\right](Z)=\sum_{\substack{T \in \mathscr{S} \\ t_{2}, t_{4}=0(\bmod N)}} A(T) e\left(\frac{\operatorname{tr}(T Z)}{N}\right)
$$

where $T=\left(\begin{array}{cc}t_{1} & t_{2} / 2 \\ t_{2} / 2 & t_{4}\end{array}\right)$. Indeed, for convergence we require $A(T)=0$ if $\operatorname{det} T<0$ and $\Phi\left(F \mid\left[\iota_{1}(J)\right]\right)=0$ implies that $A(T)=0$ if $\operatorname{det} T=0$. Similarly, we have

$$
\iota_{2}(-J) \Gamma_{0}(N) \iota_{2}(J) \equiv\left(\begin{array}{cccc}
* & * & * & * \\
0 & * & * & 0 \\
0 & * & * & 0 \\
* & * & * & *
\end{array}\right)(\bmod N) .
$$

Consequently, $F \mid\left[\iota_{2}(J)\right]$ possesses the following expansion:

$$
F \mid\left[\iota_{2}(J)\right](Z)=\sum_{\substack{T \in \mathscr{S} \\ t_{1}, t_{2}=0(\bmod N)}} A(T) e\left(\frac{\operatorname{tr}(T Z)}{N}\right) .
$$

These considerations give rise to the following lemma:

Lemma 39. Let $G \mid\left[\iota_{1}\right](Z)=\sum_{T \in \mathscr{S}} g_{1}(T)\left(\frac{\operatorname{tr}(T Z)}{N}\right)$ with $t_{2}, t_{4} \equiv 0(\bmod N)$. Then,

$$
\left.\sum_{\gamma \in R(1)} \int_{F}|G|[\gamma](Z)\right|^{2}(\operatorname{det} Y)^{k-3} d X d Y \ll N \sum_{T \in \mathscr{S}}\left|g_{1}(T)\right|^{2}\left(\frac{N^{2}}{\operatorname{det} T}\right)^{k-\frac{3}{2}}
$$

where the entries $t_{1}, t_{2}, t_{4}$ of $T$ run over $t_{1}, t_{2}, t_{4} \ll N^{1+\epsilon}$ and $t_{2}, t_{4} \equiv 0(\bmod N)$. Moreover, for $G \mid\left[\iota_{2}\right](Z)=\sum_{T \in \mathscr{S}} g_{2}(T) e\left(\frac{\operatorname{tr}(T Z)}{N}\right)$ with $N\left|t_{1}, N\right| t_{2}$ it holds

$$
\left.\sum_{\gamma \in R(2)} \int_{F}|G|[\gamma](Z)\right|^{2}(\operatorname{det} Y)^{k-3} d X d Y \ll N \sum_{u=0}^{N-1} \sum_{T \in \mathscr{S}}\left|g_{2}\left(U T U^{T}\right)\right|^{2}\left(\frac{N^{2}}{\operatorname{det} T}\right)^{k-\frac{3}{2}}
$$

where $t_{1}, t_{2}, t_{4} \ll N^{1+\epsilon}, t_{1}, t_{2} \equiv 0(\bmod N)$ and $U=\left(\begin{array}{ll}1 & u \\ & 1\end{array}\right)$. 
Proof. In the $R(1)$ case the integral equals

$$
\begin{aligned}
& \int_{Y} \sum_{\substack{T, T^{\prime} \in \mathscr{S} \\
t_{2}, t_{4}, t_{2}^{\prime}, t_{4}^{\prime}=0(\bmod N)}} g_{1}(T) \overline{A\left(T^{\prime}\right)} \exp \left(\frac{-2 \pi \operatorname{tr}\left(\mathrm{T}+\mathrm{T}^{\prime}\right) \mathrm{Y}}{N}\right)(\operatorname{det} Y)^{k-3} d Y \\
& \times \int_{0}^{N} \int_{0}^{1} \int_{0}^{1} e\left(\frac{\left(t_{1}-t_{1}^{\prime}\right) x_{1}}{N}+\frac{\left(t_{2}-t_{2}^{\prime}\right) x_{2}}{N}+\frac{\left(t_{4}-t_{4}^{\prime}\right) x_{4}}{N}\right) d x_{1} d x_{2} d x_{4} .
\end{aligned}
$$

The latter integral vanishes unless $t_{1}=t_{1}^{\prime}, t_{2}=t_{2}^{\prime}$ and $t_{4}=t_{4}^{\prime}$ in which case it equals $N$. It remains to compute

$$
N \int_{\sqrt{3} / 2}^{\infty} \int_{-y_{1} / 2}^{y_{1} / 2} \int_{y_{1}}^{\infty} \sum_{\substack{T \in \mathscr{S} \\ t_{2}, t_{4}=0(\bmod N)}}\left|g_{1}(T)\right|^{2} \exp \left(-4 \pi\left(\frac{\operatorname{tr} T Y}{N}\right)\right)(\operatorname{det} Y)^{k-3} d Y .
$$

The very same integral appears in the proof of Lemma 38 and is computed there. This yields the first claim.

In the $R(2)$ case the integral equals

$$
\int_{Y} \int_{\substack{x_{1}, x_{2} \in[0,1] \\ x_{4} \in[0, N]}} \sum_{U}|G|\left[\iota_{2}(J)\right]\left(U^{T} Z U\right) \mid(\operatorname{det} Y)^{k-3} d X d Y .
$$

where $U$ runs over $\left(\begin{array}{cc}1 & u \\ & 1\end{array}\right)$ with $0 \leqslant u \leqslant N-1$. For $U T U^{T}=\left(\begin{array}{cc}\tilde{t}_{1} & \tilde{t}_{2} \\ \tilde{t}_{2} & \tilde{t}_{4}\end{array}\right)$ we cannot assume that $\tilde{t}_{1}, \tilde{t}_{2} \equiv 0(\bmod N)$. For that reason, we consider $N^{2}$ copies of the fundamental domain $F$ by letting $x_{1}, x_{2} \in[0, N]$. Then, the previous display equals

$$
\left.\frac{1}{N^{2}} \int_{Y} \int_{X \in \mathcal{P}(\mathbb{Z} / N \mathbb{Z})} \sum_{U}|G|\left[\iota_{2}(J)\right]\left(U^{T} Z U\right)\right|^{2}(\operatorname{det} Y)^{k-3} d X d Y .
$$

This is bounded by

$$
N \sum_{U} \int_{\sqrt{3} / 2}^{\infty} \int_{-y_{1} / 2}^{y_{1} / 2} \int_{y_{1}}^{\infty} \sum_{\substack{T \in \mathscr{S} \\ t_{2}, t_{4} \equiv 0(\bmod N)}}\left|g_{2}\left(U T U^{T}\right)\right|^{2} \exp \left(-4 \pi\left(\frac{\operatorname{tr} T Y}{N}\right)\right)(\operatorname{det} Y)^{k-3} d Y .
$$

Computing the integral as in the proof of Lemma 38 yields the second claim.

In summary, it remains to evaluate the Fourier coefficients of $G, G\left|\left[\iota_{1}\right], G\right|\left[\iota_{2}\right]$ and $G \mid\left[J_{4}\right]$ in order to obtain an upper bound for $\langle G, G\rangle$. As the estimation of Fourier coefficients of Klingen-Eisenstein and theta series is based on different methods, we treat them one by one. We start with the more challenging Klingen-Eisenstein part. 


\subsubsection{Decomposition of matrices in $\mathrm{Sp}_{4}(\mathbb{Z})$ and $\Gamma_{0}^{(2)}(N)$}

The aim of this section is to find a decomposition of $P(N) \backslash \Gamma_{0}^{(2)}(N) R$ in order to compute

$$
A_{R}(T)=\int_{X \in \mathcal{P}(\mathbb{R}) / \mathcal{P}(N \mathbb{Z})} \sum_{M \in P(N) \backslash \Gamma_{0}^{(2)}(N) R} f(\pi(M Z)) j(M, Z)^{-k} e\left(-\frac{\operatorname{tr}(T X)}{N}\right)
$$

for $T>0$. We start by showing that some matrices in the sum over $M$ do not contribute to the Fourier coefficients $A_{R}(T)$ if $\operatorname{det} T \neq 0$. Then, we find a suitable decomposition for the remaining matrices. To do so, we closely follow the work from Kitaoka for the principal congruence subgroup, cf. [31] and [34].

Let $\lambda(M)$ denote the bottom row of a matrix $M$. For a better understanding, we state and prove [31, Proposition 1.6.12]:

Lemma 40. Let $f \in S_{k}(N)$ and $R \in \mathrm{Sp}_{4}(\mathbb{Z})$. The Fourier coefficients of

$$
\sum_{\substack{M \in P(N) \backslash \Gamma_{0}^{(2)}(N) R \\ \lambda(M)=(0,0, *, *)}} j_{M}(Z)^{-k} f(\pi(M(Z)))
$$

vanish for $T>0$.

Proof. First, recall that $\Gamma(\mathrm{N})=\left\{M \in \mathrm{Sp}_{4}(\mathbb{Z}) \mid M \equiv I_{4}(\bmod N)\right\}$ is a normal subgroup of $\mathrm{Sp}_{4}(\mathbb{Z})$. Hence, we have a Fourier extension

$$
\sum_{\substack{M \in P(N) \backslash \Gamma_{0}^{(2)}(N) R \\ \lambda(M)=(0,0, *, *)}} j_{M}(Z)^{-k} f(\pi(M(Z)))=\sum_{T \in \mathcal{S}} a(T) e\left(\frac{\operatorname{tr} T Z}{N}\right) .
$$

For $M \in \operatorname{Sp}_{4}(\mathbb{Z})$ with $\lambda(M)=\left(0,0, d_{3}, d_{4}\right)$ set $u_{1}=\frac{-d_{4}}{\left(d_{3}, d_{4}\right)}, u_{2}=\frac{d_{3}}{\left(d_{3}, d_{4}\right)}$ and consider $U=\left(\begin{array}{cc}u_{1} & u_{2} \\ * & *\end{array}\right) \in \mathrm{SL}_{2}(\mathbb{Z})$. Then,

$$
M\left(\begin{array}{cc}
U^{-1} & \\
& U^{T}
\end{array}\right) \in P(\mathbb{Z}) .
$$

By (72) we have

$$
\begin{aligned}
j(M, Z) & =j(U, Z) j\left(L, U Z U^{T}\right)=d_{4}\left(c_{1} \pi\left(U Z U^{T}\right)+d_{1}\right), \\
\pi(M Z) & =\omega(L) \pi\left(U Z U^{T}\right),
\end{aligned}
$$

for some $L \in P(\mathbb{Z})$ whose lower rows are given by $\left(c_{1}, 0, d_{1}, *\right)$ and $\left(0,0,0, d_{4}\right)$. 
Moreover, we set

$$
\frac{\partial}{\partial Y}=\left(\begin{array}{cc}
\frac{\partial}{\partial y_{1}} & \frac{1}{2} \frac{\partial}{\partial y_{2}} \\
\frac{1}{2} \frac{\partial}{\partial y_{2}} & \frac{\partial}{\partial y_{4}}
\end{array}\right) \quad \text { for } \quad Y=\left(\begin{array}{ll}
y_{1} & y_{2} \\
y_{2} & y_{4}
\end{array}\right)=\operatorname{Im} Z
$$

By $D_{Y}$ we denote the differential operator

$$
D_{Y}=(\operatorname{det} Y)\left(\operatorname{det} \frac{\partial}{\partial Y}\right) .
$$

It is straightforward to show that $D_{Y}=D_{V Y V^{T}}$ for $V \in \mathrm{GL}_{2}(\mathbb{R})$. Hence,

$$
\begin{aligned}
D_{Y}\left(j(M, Z)^{-k} f(\pi(M(Z)))\right) & =d_{4} D_{Y}(f \mid[\omega(L)])\left(\pi\left(U Z U^{T}\right)\right) \\
& =d_{4} D_{U Y U^{T}}(f \mid[\omega(L)])\left(\pi\left(U X U^{T}+i U Y U^{T}\right)\right) .
\end{aligned}
$$

By substituting $Y=U Y U^{T}$ the previous display equals

$$
d_{4} \operatorname{det} Y \operatorname{det}\left(\begin{array}{ll}
* & 0 \\
0 & 0
\end{array}\right)=0
$$

since $(f \mid[\omega(L)])\left(\pi\left(U X U^{T}+i Y\right)\right.$ only depends on $y_{1}$ and not on $y_{2}$ or $y_{4}$. On the other hand, we have

$$
\left(\operatorname{det} \frac{\partial}{\partial Y} e\left(\frac{\operatorname{tr}(T Z))}{N}\right)\right)=\frac{4 \pi^{2}}{N^{2}}(\operatorname{det} T) e\left(\frac{\operatorname{tr}(T Z)}{N}\right) .
$$

Thus, by letting $D_{Y}$ act on both sides of 89 , we get

$$
0=D_{Y}\left(\sum_{T \in \mathcal{S}} a(T) e\left(\frac{\operatorname{tr}(T Z)}{N}\right)\right)=\frac{4 \pi^{2}}{N^{2}}(\operatorname{det} Y) \sum_{T \in \mathcal{S}} a(T)(\operatorname{det} \mathrm{T}) e(\operatorname{tr}(\mathrm{TZ}))
$$

This implies that $a(T)=0$ for $T>0$.

Consequently, we may neglect matrices $M$ with $\lambda(M)=(0,0, *, *)$ in our decomposition and only consider $M \in P(N) \backslash \Gamma_{0}^{(2)}(N)$ with $\lambda(M) \neq(0,0, *, *)$. For this purpose, the first step is to decompose $M \in P(\mathbb{Z}) \backslash \operatorname{Sp}_{4}(\mathbb{Z})$ with $\lambda(M) \neq(0,0, *, *)$. Therefore, we follow Kitaokas's work [34.

For this purpose, we introduce further notation. Set $\mathrm{PSL}_{2}(\mathbb{Z})=\mathrm{SL}_{2}(\mathbb{Z}) /\{ \pm 1\}$ and for a subgroup $\Gamma$ of $\mathrm{SL}_{2}(\mathbb{Z})$ we denote by $\bar{\Gamma}=\Gamma\{( \pm 1\} /\{ \pm 1\}$ the image of $\Gamma$ in $\operatorname{PSL}_{2}(\mathbb{Z})$. Furthermore, for $\sigma=\left(\begin{array}{ll}a & b \\ c & d\end{array}\right)$ we set

$$
\iota_{1}(\sigma)=\left(\begin{array}{cccc}
a & & b & \\
& 1 & & 0 \\
c & & d & \\
& 0 & & 1
\end{array}\right) \text { and } \iota_{2}(\sigma)=\left(\begin{array}{cccc}
1 & & 0 & \\
& a & & b \\
0 & & 1 & \\
& c & & d
\end{array}\right) \text {. }
$$


Then, $\iota_{i}(\sigma)^{-1}=\iota_{i}\left(\sigma^{-1}\right)$ and for $\sigma \in \Gamma_{0}(N)$ we have that $\iota_{1}(\sigma), \iota_{2}(\sigma) \in \Gamma_{0}^{(2)}(N)$. Moreover, we put

$$
\gamma(v, \sigma):=\left(\begin{array}{cc}
v & \\
& v^{-T}
\end{array}\right) \iota_{2}(\sigma) \in \operatorname{Sp}_{4}(\mathbb{Z})
$$

for $\sigma, v \in \mathrm{SL}_{2}(\mathbb{Z})$ and set $\Gamma_{\infty}(a)=\left(\begin{array}{cc}1 & a \mathbb{Z} \\ 0 & 1\end{array}\right)$ as well as $\Gamma_{\infty}:=\Gamma_{\infty}(1)$. The following two lemmas are based on [34, Lemma $2 \& 3]$ :

Lemma 41. For $M \in \operatorname{Sp}_{4}(\mathbb{Z})$ with $\lambda(M)=\left(0, c_{4}, d_{3}, d_{4}\right)$ and $c_{4} \neq 0$ there are (unique) $v=\left(\begin{array}{cc}v_{1} & v_{2} \\ * & *\end{array}\right) \in \Gamma_{\infty}^{T} \backslash \mathrm{SL}_{2}(\mathbb{Z})$ with $v_{1}>0$ and $\sigma=\left(\begin{array}{ll}* & * \\ c & d\end{array}\right) \in \Gamma_{\infty} \backslash \mathrm{SL}_{2}(\mathbb{Z})$ with $c>0$ such that

$$
M \in P(\mathbb{Z}) \gamma(v, \sigma) .
$$

Proof. By multiplying $M$ with $-I_{4} \in P(\mathbb{Z})$, we may assume that $c_{4}>0$. We set $\tilde{u}_{4}=\left(c_{4}, d_{4}\right), c=\frac{c_{4}}{\tilde{u_{4}}}, d=\frac{d_{4}}{\tilde{u}_{4}}$ and choose any $a, b$ such that

$$
\sigma:=\left(\begin{array}{ll}
a & b \\
c & d
\end{array}\right) \in \mathrm{SL}_{2}(\mathbb{Z})
$$

The last row of $M \iota_{2}(\sigma)^{-1}$ is given by

$$
\left(0, c_{4}, d_{3}, d_{4}\right)\left(\begin{array}{cccc}
1 & & 0 & \\
& d & & -b \\
0 & & 1 & \\
& -c & & a
\end{array}\right)=\left(0,0, d_{3}, \tilde{u}_{4}\right)
$$

As the matrix above is in $\operatorname{Sp}_{4}(\mathbb{Z})$ we infer that $d_{3}$ and $\tilde{u}_{4}$ are coprime. Hence, there is an extension

$$
\tilde{u}=\left(\begin{array}{cc}
* & * \\
d_{3} & \tilde{u}_{4}
\end{array}\right) \in \mathrm{SL}_{2}(\mathbb{Z})
$$

We write $\tilde{u}=w u$ with $w \in \Gamma_{\infty}$ and $u \in \Gamma_{\infty} \backslash \mathrm{SL}_{2}(\mathbb{Z})$. Then,

$$
\lambda\left(M \iota_{2}(\sigma)^{-1}\left(\begin{array}{ll}
u^{T} & \\
& u^{-1}
\end{array}\right)\right)=(0,0,0, *)\left(\begin{array}{ll}
w^{-T} & \\
& w
\end{array}\right)=(0,0,0, *) .
$$

Thus, $M \iota_{2}(\sigma)^{-1}\left(\begin{array}{cc}u^{T} & \\ & u^{-1}\end{array}\right) \in P(\mathbb{Z})$. By setting $v=u^{-T}$, we conclude that

$$
M \in P(\mathbb{Z})\left(\begin{array}{ll}
v & \\
& v^{-T}
\end{array}\right) \iota_{2}(\sigma)^{-1}=P(\mathbb{Z}) \gamma(v, \sigma) .
$$

Note that $v_{1}=u_{4}=\tilde{u}_{4}>0$. Furthermore, we can assume by left multiplication with $P(\mathbb{Z})$ that $\sigma \in \Gamma_{\infty} \backslash \mathrm{SL}_{2}(\mathbb{Z})$ since the upper entries of $\sigma$ have no impact on the lower row of the decomposition. 
Lemma 42. We get the following disjoint decomposition:

$$
\left\{M \in \operatorname{Sp}_{4}(\mathbb{Z}) \mid \lambda(M) \neq(0,0, *, *)\right\}=\bigsqcup_{\sigma, v, w} P(\mathbb{Z}) \gamma(v, \sigma)\left(\begin{array}{ll}
w^{-1} & \\
& w^{T}
\end{array}\right)
$$

where

$$
\sigma=\left(\begin{array}{ll}
* & * \\
c & d
\end{array}\right) \in \Gamma_{\infty} \backslash \mathrm{SL}_{2}(\mathbb{Z}), v=\left(\begin{array}{cc}
v_{1} & v_{2} \\
* & *
\end{array}\right) \in \Gamma_{\infty}^{T} \backslash \mathrm{SL}_{2}(\mathbb{Z}), w \in \mathrm{PSL}_{2}(\mathbb{Z}) / \bar{\Gamma}_{\infty}
$$

with $c>0$ and $v_{1}>0$.

Proof. For $M=\left(\begin{array}{ll}A & B \\ C & D\end{array}\right) \in \operatorname{Sp}_{4}(\mathbb{Z})$ with $\lambda(M) \neq(0,0, *, *)$, we choose $w \in$ $\operatorname{PSL}_{2}(\mathbb{Z}) / \bar{\Gamma}_{\infty}$ such that

$$
C w=\left(\begin{array}{cc}
* & * \\
0 & c_{4}
\end{array}\right) \quad \text { with } c_{4} \neq 0
$$

This allows us to apply Lemma 41 for $M\left(\begin{array}{ll}w & \\ & w^{-T}\end{array}\right)$ which implies that $M$ is contained in the right hand side of $(90)$.

Conversely, assume that

$$
M=L \gamma(v, \sigma)\left(\begin{array}{ll}
w^{-1} & \\
& w^{T}
\end{array}\right)
$$

where $L \in P(\mathbb{Z})$. Denote the upper left entry of $\sigma$ by $a$ and the penultimate row of $L$ by $\left(c_{1}, 0, d_{1}, d_{2}\right)$. Then, the lower left two-by-two block of $M$ is given by

$$
\left(\left(\begin{array}{cc}
c_{1} & 0 \\
0 & 0
\end{array}\right) v\left(\begin{array}{ll}
1 & 0 \\
0 & a
\end{array}\right)+\left(\begin{array}{cc}
d_{1} & d_{2} \\
0 & \pm 1
\end{array}\right) v^{-T}\left(\begin{array}{ll}
0 & 0 \\
0 & c
\end{array}\right)\right) w^{-1}=\left(\begin{array}{cc}
* & * \\
0 & \pm v_{1} c
\end{array}\right) w^{-1}
$$

As $c, v_{1} \neq 0$ this implies that $\lambda(M) \neq(0,0, *, *)$.

It remains to show that the decomposition is disjoint. Therefore, we fix representatives of the cosets $\Gamma_{\infty} \backslash \mathrm{SL}_{2}(\mathbb{Z}), \Gamma_{\infty}^{T} \backslash \mathrm{SL}_{2}(\mathbb{Z}), \mathrm{PSL}_{2}(\mathbb{Z}) / \bar{\Gamma}_{\infty}$ and consider two pairs $\sigma, v, w$ and $\tilde{\sigma}, \tilde{v}, \tilde{w}$ of such representatives with $c>0, \tilde{c}>0, v_{1}>0, \tilde{v}_{1}>0$ that satisfy

$$
P(\mathbb{Z}) \gamma(v, \sigma)\left(\begin{array}{cc}
w^{-1} & \\
& w^{T}
\end{array}\right)=P(\mathbb{Z}) \gamma(\tilde{v}, \tilde{\sigma})\left(\begin{array}{cc}
\tilde{w}^{-1} & \\
& \tilde{w}^{T}
\end{array}\right)
$$

Since

$$
\gamma(v, \sigma)=\left(\begin{array}{cccc}
* & * & * & * \\
0 & v_{1} c & -v_{3} & v_{1} d
\end{array}\right)
$$


it follows by equation (91) that

$$
\left(0, v_{1} c,-v_{3}, v_{1} d\right)= \pm\left(0, \tilde{v}_{1} \tilde{c},-\tilde{v}_{3}, \tilde{v}_{1} \tilde{d}\right)\left(\begin{array}{ll}
\tilde{w}^{-1} w & \\
& \left(\tilde{w}^{-1} w\right)^{T}
\end{array}\right)
$$

By

$$
\left(0, v_{1} c\right) w^{-1} \tilde{w}= \pm\left(0, \tilde{v}_{1} \tilde{c}\right)
$$

and by $v_{1} c \neq 0$, we conclude that $w^{-1} \tilde{w} \in \bar{\Gamma}_{\infty}$ and thus $w=\tilde{w}$ since they are part of the same class. This, on the other hand implies

$$
v_{1}(c, d)=\tilde{v}_{1}(\tilde{c}, \tilde{d})
$$

and hence, $v_{1}=\tilde{v}_{1}, \sigma \tilde{\sigma}^{-1} \in \Gamma_{\infty}$ and thus, $\sigma=\tilde{\sigma}$. As a consequence, we get $v_{3}=\tilde{v}_{3}$ which implies that $\tilde{v}^{-1} v \in \Gamma_{\infty}$.

The next result, based on [34, Lemma 4], plays a crucial part in the computation of the Fourier coefficients:

Lemma 43. For $\mu=\left(\begin{array}{ll}\mu_{1} & \mu_{2} \\ \mu_{2} & \mu_{4}\end{array}\right) \in M_{2}(\mathbb{Z})$ and $\sigma, v \in \mathrm{SL}_{2}(\mathbb{Z})$ we have that

$$
\gamma(v, \sigma)\left(\begin{array}{cc}
I & \mu \\
0 & I
\end{array}\right)=\left(\begin{array}{ll}
I & \tau \\
0 & I
\end{array}\right) \gamma\left(v\left(\begin{array}{cc}
1 & -\mu_{2} c \\
0 & 1
\end{array}\right), \sigma\left(\begin{array}{cc}
1 & \mu_{4} \\
0 & 1
\end{array}\right)\right)
$$

where $a$ and $c$ are the upper and lower left entries of $\sigma$ and

$$
\tau=v\left(\begin{array}{cc}
\mu_{1}-\mu_{2}^{2} a c & \mu_{2} a \\
a \mu_{2} & 0
\end{array}\right) v^{T} .
$$

Proof. We set $\tilde{v}=v\left(\begin{array}{cc}1 & -\mu_{2} c \\ 0 & I\end{array}\right)$. Then,

$$
\begin{aligned}
& \gamma(v, \sigma)\left(\begin{array}{cc}
I & \mu \\
0 & I
\end{array}\right) \gamma\left(\tilde{v}, \sigma\left(\begin{array}{cc}
1 & \mu_{4} \\
0 & I
\end{array}\right)\right)^{-1} \\
& =\left(\begin{array}{ll}
v & \\
& v^{-T}
\end{array}\right) \iota_{2}(\sigma)\left(\begin{array}{cccc}
1 & & \mu_{1} & \mu_{2} \\
& 1 & \mu_{2} & \\
& & 1 & \\
& & & 1
\end{array}\right) \iota_{2}\left(\sigma^{-1}\right)\left(\begin{array}{cc}
\tilde{v} & \\
& \tilde{v}^{-T}
\end{array}\right) .
\end{aligned}
$$

A simple calculation shows that

$$
\iota_{2}(\sigma)\left(\begin{array}{cccc}
1 & & \mu_{1} & \mu_{2} \\
& 1 & \mu_{2} & \\
& & 1 & \\
& & & 1
\end{array}\right) \iota_{2}\left(\sigma^{-1}\right)=\left(\begin{array}{cccc}
1 & -\mu_{2} c & \mu_{1} & \mu_{2} a \\
& 1 & a \mu_{3} & \\
& & 1 & \\
& & \mu_{2} c & 1
\end{array}\right) .
$$


Hence 92 is equal to

$$
\begin{aligned}
& \left(\begin{array}{cc}
v & \\
& v^{-T}
\end{array}\right)\left(\begin{array}{cccc}
1 & -\mu_{2} c & \mu_{1} & \mu_{2} a \\
& 1 & a \mu_{3} & \\
& & 1 & \\
& & \mu_{2} c & 1
\end{array}\right)\left(\begin{array}{cccc}
1 & \mu_{2} c & & \\
& 1 & & \\
& & 1 & \\
& & -\mu_{2} c & 1
\end{array}\right)\left(\begin{array}{cc}
v^{-1} & \\
& v^{T}
\end{array}\right) \\
& =\left(\begin{array}{cc}
v & \\
& v^{-T}
\end{array}\right)\left(\begin{array}{cc}
I & v^{-1} \tau v^{-T} \\
& I
\end{array}\right)\left(\begin{array}{cc}
v^{-1} & \\
& v^{T}
\end{array}\right)=\left(\begin{array}{ll}
I & \tau \\
& I
\end{array}\right) .
\end{aligned}
$$

This gives a second decomposition of $M \in \operatorname{Sp}_{4}(\mathbb{Z})$ with $\lambda(M) \neq(0,0, *, *)$ which turns out to be very useful for the evaluation of Fourier coefficients of KlingenEisenstein series:

Lemma 44. Fix any two positive integers $N_{1}, N_{2}$. There is a disjoint decomposition

$$
\left\{M \in \operatorname{Sp}_{4}(\mathbb{Z}) \mid \lambda(M) \neq(0,0, *, *)\right\}=\bigsqcup_{v, \sigma, \mu, w} P(\mathbb{Z}) \gamma(v, \sigma)\left(\begin{array}{cc}
I & \mu \\
& I
\end{array}\right)\left(\begin{array}{ll}
w^{-1} & \\
& w^{T}
\end{array}\right)
$$

with

$$
\begin{aligned}
\mu & =\left(\begin{array}{cc}
0 & \mu_{2} \\
\mu_{2} & \mu_{4}
\end{array}\right) \text { with } \mu_{2} \in N_{1} \mathbb{Z}, \mu_{4} \in N_{2} \mathbb{Z}, \\
\sigma & =\left(\begin{array}{ll}
a & b \\
c & d
\end{array}\right) \in \Gamma_{\infty} \backslash \mathrm{SL}_{2}(\mathbb{Z}) / \Gamma_{\infty}\left(N_{2}\right), c>0, \\
v & =\left(\begin{array}{ll}
v_{1} & v_{2} \\
v_{3} & v_{4}
\end{array}\right) \in \Gamma_{\infty}^{T} \backslash \mathrm{SL}_{2}(\mathbb{Z}) / \Gamma_{\infty}\left(N_{1} c\right), v_{1}>0, \\
w & =\left(\begin{array}{ll}
w_{1} & w_{2} \\
w_{3} & w_{4}
\end{array}\right) \in \operatorname{PSL}_{2}(\mathbb{Z}) / \bar{\Gamma}_{\infty} .
\end{aligned}
$$

Proof. Set $\mathrm{Sp}_{4}(\mathbb{Z})^{*}=\left\{M \in \mathrm{Sp}_{4}(\mathbb{Z}) \mid \lambda(M) \neq(0,0, *, *)\right\}$. By Lemma 42 , we get a decomposition

$$
\mathrm{Sp}_{4}(\mathbb{Z})^{*}=\bigsqcup_{\sigma, v, \mu, w} P(\mathbb{Z}) \gamma\left(v\left(\begin{array}{cc}
1 & -\mu_{2} c \\
0 & 1
\end{array}\right), \sigma\left(\begin{array}{cc}
1 & \mu_{4} \\
0 & 1
\end{array}\right)\right)\left(\begin{array}{ll}
w^{-1} & \\
& w^{T}
\end{array}\right)
$$

with $\sigma, v, \mu, w$ as above. By applying Lemma 43 we obtain the desired decomposition. It remains to show the uniqueness. Therefore, assume that

$$
\Gamma_{\infty} \sigma=\Gamma_{\infty} \sigma\left(\begin{array}{cc}
1 & \mu_{4} \\
0 & 1
\end{array}\right)
$$


Then

$$
\Gamma_{\infty} \ni \sigma\left(\begin{array}{cc}
1 & \mu_{4} \\
0 & 1
\end{array}\right) \sigma^{-1}=\left(\begin{array}{cc}
* & * \\
-c^{2} \mu_{4} & *
\end{array}\right)
$$

implies that $\mu_{4}=0$ since $c \neq 0$. In a similar manner, $\lambda_{2}=0$ if $\Gamma_{\infty}^{T} v\left(\begin{array}{cc}1 & \lambda_{2} \\ 0 & 1\end{array}\right)=\Gamma_{\infty}^{T} v$ since

$$
\Gamma_{\infty}^{T} \ni v\left(\begin{array}{cc}
1 & \lambda_{2} \\
0 & 1
\end{array}\right) v^{-1}=\left(\begin{array}{cc}
* & v_{1}^{2} \lambda_{2} \\
* & *
\end{array}\right)
$$

and $v_{1} \neq 0$.

Kitaoka extends this to a decomposition with respect to the principal congruence subgroup. This approach is also useful to determine a decomposition of $M \in$ $P(N) \backslash \Gamma_{0}^{(2)}(N) R$ with $R \in \operatorname{Sp}_{4}(\mathbb{Z})$ and $\lambda(M) \neq(0,0, *, *)$. To do so, we start with the decomposition above for $P(\mathbb{Z}) \backslash \mathrm{Sp}_{4}(\mathbb{Z})$ and multiply if from the right hand side by $R^{-1}$. This gives a decomposition of

$$
\left\{M \in P(\mathbb{Z}) \backslash \mathrm{Sp}_{4}(\mathbb{Z}) \mid \lambda(M) \neq(0,0, *, *) R^{-1}\right\} .
$$

To restrict this to $\Gamma_{0}^{(2)}(N)$, we write $P(\mathbb{Z})=P(N) L$, where $L$ runs over a set of representatives in $P(\mathbb{Z}) \backslash P(N)$ and introduce congruence conditions for the different components such that each element is contained in $\Gamma_{0}^{(2)}(N)$. As a consequence, we obtain a decomposition of $P(N) \backslash \Gamma_{0}^{(2)}(N)$ with $\lambda(M) \neq(0,0, *, *) R^{-1}$. Multiplying from the right-hand side by $R$ we obtain the following result:

Corollary 45. Let

$$
\mathcal{M}=\bigsqcup_{v, \sigma, \mu, w} P(N) L \gamma(v, \sigma)\left(\begin{array}{cc}
I & \mu \\
& I
\end{array}\right)\left(\begin{array}{ll}
w^{-1} & \\
& w^{T}
\end{array}\right)
$$

with $v, \sigma, \mu, w$ as in Lemma 44 and $L$ running over a set of representatives in $P(N) \backslash P(\mathbb{Z})$. Let $(\#)$ denote a set of congruences such that $\mathcal{M} R^{-1} \subset \Gamma_{0}^{(2)}(N)$ if and only if (\#) is satisfied. Then, we have a disjoint decomposition

$$
\left\{M \in \Gamma_{0}^{(2)}(N) R \mid \lambda(M) \neq(0,0, *, *)\right\}=\{\mathcal{M} \mid(\#)\} .
$$

Note that every set of representatives in $P(N) \backslash P(\mathbb{Z})$ corresponds to a set of representatives in $\Gamma_{0}(N) \backslash \mathrm{SL}_{2}(\mathbb{Z})$ by the map $(71)$. Furthermore, if $\gamma_{i}$ is a set of representatives in $\Gamma_{0}(N) \backslash \mathrm{SL}_{2}(\mathbb{Z})$, then $\iota_{1}\left(\gamma_{i}\right)$ is a set of representatives in $P(N) \backslash P(\mathbb{Z})$. Recall that for $N$ prime a set of representatives of $\Gamma_{0}(N) \backslash \mathrm{SL}_{2}(\mathbb{Z})$ is given by

$$
\left\{I, J\left(\begin{array}{cc}
1 & d_{1} \\
& 1
\end{array}\right) \mid 0 \leqslant d_{1} \leqslant N-1\right\} .
$$

To determine a suitable decomposition for all $M \in \Gamma_{0}(N)$ with $\lambda(M) \neq(0,0, *, *)$ we apply the procedure of Corollary 45 for $R=I_{4}$ : 
Lemma 46. We have

$$
\left\{M \in \Gamma_{0}^{(2)}(N) \mid \lambda(M) \neq(0,0, *, *)\right\}=\bigsqcup_{v, \sigma, \mu, w} P(N) L \gamma(v, \sigma)\left(\begin{array}{cc}
I & \mu \\
0 & I
\end{array}\right)\left(\begin{array}{ll}
w^{-1} & \\
& w^{T}
\end{array}\right)
$$

with $v, \sigma, \mu, w$ as in Lemma 44 and either

$$
N \mid c \quad \text { or } \quad N \mid v_{1}, N \nmid c .
$$

In the former case we put $L=I_{4}$, and in the latter case

$$
L=\iota_{1}\left(J\left(\begin{array}{cc}
1 & d_{1} \\
& 1
\end{array}\right)\right)
$$

for $d_{1} \equiv-a v_{2}^{2} \bar{c}(\bmod N)$ with $\bar{c}$ given by $c \bar{c} \equiv 1(\bmod N)$.

Proof. By Corollary 45 we consider

$$
\bigsqcup_{\sigma, v, \mu, w, L} P(N) L \gamma(v, \sigma)\left(\begin{array}{cc}
I_{2} & \mu \\
0 & I_{2}
\end{array}\right)\left(\begin{array}{ll}
w^{-1} & \\
& w^{T}
\end{array}\right),
$$

for some $L \in P(N) \backslash P(\mathbb{Z})$ and examine the necessary conditions for this decomposition to be in $\Gamma_{0}^{(2)}(N)$. If we write the penultimate row of $L$ as $\left(c_{1}, 0, d_{1}, d_{2}\right)$, this is the case if and only if

$$
\left(\begin{array}{cc}
c_{1} v_{1} & c_{1} v_{2} a-d_{1} v_{3} c+d_{2} v_{1} c \\
0 & \pm v_{1} c
\end{array}\right) . \equiv 0 \quad(\bmod N)
$$

This implies that $N \mid c$ or $N \mid v_{1}$. In the former case, it holds by the two upper entries that $C \equiv 0(\bmod N)$ if and only if $c_{1} \equiv 0(\bmod N)$. This implies that $L \in P(N)$ and we choose $L=I_{4}$.

For $N \mid v_{1}$, we require $a c_{1} v_{2} \equiv d_{1} c v_{3} \bmod N$. If $N \mid c$, this implies that $N \mid c_{1}$ and we put $L=I_{4}$. If $N \nmid c$, then any $c_{1} \equiv e c v_{3} \bmod N$ and $d_{1} \equiv e a v_{2} \bmod N$ for some $(e, N)=1$ and $\left(c_{1}, d_{1}\right)=1$ satisfy this condition. All these matrices lie in the same coset of $P(N) \backslash P(\mathbb{Z})$ and making use of $(93)$ we choose

$$
L=\iota_{1}\left(J\left(\begin{array}{cc}
1 & d_{1} \\
& 1
\end{array}\right)\right)
$$

with $d_{1} \equiv a v_{2} \overline{c v_{3}} \equiv-a v_{2}^{2} \bar{c}(\bmod N)$.

For the Eisenstein series $E_{N}\left(f_{2}, Z\right)$, the sum runs over matrices $M \in \Gamma_{0}(N)$ with $\lambda\left(M J_{4}\right) \neq(0,0, *, *)$. To this end, we derive the following decomposition: 
Lemma 47. We have

$$
\left\{M \in \Gamma_{0}(N) \mid \lambda\left(M J_{4}\right) \neq(0,0, *, *)\right\}=\bigsqcup_{v, \sigma, \mu, w} P(N) \iota_{1}(J) \gamma(v, \sigma)\left(\begin{array}{cc}
I & \mu \\
0 & I
\end{array}\right)\left(\begin{array}{ll}
w^{-1} & \\
& w^{T}
\end{array}\right)
$$

with $\mu_{2}, \mu_{4} \in N \mathbb{Z}, v, \sigma, \mu, w$ as in Lemma 44.

$$
N \mid d \text { and } N \mid v_{2} \text {. }
$$

Proof. By Corollary 45 we need to examine the condition under which

$$
\begin{aligned}
& \bigsqcup_{v, \sigma, \mu, w} P(N) L \gamma(v, \sigma)\left(\begin{array}{ll}
I & \mu \\
0 & I
\end{array}\right)\left(\begin{array}{ll}
w^{-1} & \\
& w^{T}
\end{array}\right)\left(\begin{array}{ll} 
& -I \\
I &
\end{array}\right) \\
& =\bigsqcup_{v, \sigma, \mu, w} P(\mathbb{Z})\left(\begin{array}{ll}
v & \\
& v^{-T}
\end{array}\right) \iota_{1}(J) \iota_{2}(\sigma J)\left(\begin{array}{ll}
I & 0 \\
\mu & I
\end{array}\right)\left(\begin{array}{ll}
w^{T} & \\
& w^{-1}
\end{array}\right)
\end{aligned}
$$

is in $\Gamma_{0}(N)$, where $\sigma, v, w, \mu$ are as in Lemma 44 and $L$ run over $P(N) \backslash P(\mathbb{Z})$. For simplicity, we assume that $\mu_{2}, \mu_{4} \in N \mathbb{Z}$. Then, the decomposition above is contained in $\Gamma_{0}(N)$ if

$$
\left(\begin{array}{cc}
d_{1} v_{4}+d_{2} v_{2} & c_{1} v_{2} b-d_{1} v_{3} d+d_{2} v_{1} d \\
\mp v_{2} & \pm v_{1} d
\end{array}\right) \equiv 0(\bmod N)
$$

where the penultimate row of $L$ is $\left(c_{1}, 0, d_{1}, d_{2}\right)$. This congruence is satisfied if and only if $N$ divides $d_{1}, v_{2}$ and $d$. Consequently, we set $L=\iota_{1}(J)$.

To compute the inner product of the cuspidal part of the theta series, we need to examine how the slash operators $\mid\left[\iota_{1}(J)\right]$ and $\mid\left[\iota_{2}(J)\right]$ act on Klingen-Eisenstein series and its Fourier coefficients. The sums in $E\left(f_{1}, Z\right) \mid\left[\iota_{1}(J)\right]$ and $E_{N}\left(f_{2}, Z\right) \mid\left[\iota_{2}(J)\right]$ run over $M \in P(N) \backslash \Gamma_{0}(N) \iota_{1}(J)$ while in $E\left(f_{1}, Z\right) \mid\left[\iota_{2}(J)\right]$ and $E_{N}\left(f_{2}, Z\right) \mid\left[\iota_{1}(J)\right]$ they run over $M^{\prime} \in P(N) \backslash \Gamma_{0}(N) \iota_{2}(J)$. By Lemma 40 it suffices to consider $M, M^{\prime}$ such that $\lambda(M), \lambda\left(M^{\prime}\right) \neq(0,0, *, *)$. We use the decomposition from Lemma 44 with the following congruence conditions:

Lemma 48. Let

$$
\mathcal{M}=\bigsqcup_{v, \sigma, \mu, w} P(N) L \gamma(v, \sigma)\left(\begin{array}{cc}
I & \mu \\
& I
\end{array}\right)\left(\begin{array}{ll}
w^{-1} & \\
& w^{T}
\end{array}\right)
$$

with $v, \sigma, w, \mu$ as in Lemma 44 and $L \in P(\mathbb{Z})$ with $\omega(L)=\left(\begin{array}{cc}* & * \\ c_{1} & d_{1}\end{array}\right)$.

We have the following decomposition:

$$
\left\{M \in \Gamma_{0}(N) \iota_{1}(J) \mid \lambda(M) \neq(0,0, *, *)\right\}=\left\{\mathcal{M} \mid \mu_{4} \in N \mathbb{Z} ;(a) \text { or }(b) \text { or }(c) \text { or }(d)\right\}
$$


where $(a),(b),(c),(d)$ denote the following congruence conditions

(a) $v_{1}, w_{1} \equiv 0, d \not \equiv 0(\bmod N)$,

(b) $c, w_{2}, v_{2}, d_{1} \equiv 0(\bmod N)$,

(c) $c \equiv 0, w_{1}, w_{2}, v_{1}, v_{2} \not \equiv 0(\bmod N)$,

(d) $w_{1}, d, c_{1} \equiv 0(\bmod N)$.

In case $(a)$ we set $c_{1} \equiv 1$ and $d_{1} \equiv v_{2} b \overline{v_{3} d}(\bmod N)$ and in case $(c)$ we assume for simplification $\mu_{2} \in N \mathbb{Z}$ and set

$$
d \equiv v_{2} w_{1} \overline{v_{1} w_{2}}, c_{1} \equiv 1 \text { and } d_{1} \equiv v_{2} b w_{2} \overline{v_{1} w_{1}}(\bmod N) .
$$

Furthermore, we have

$\left\{M^{\prime} \in \Gamma_{0}(N) \iota_{2}(J) \mid \lambda\left(M^{\prime}\right) \neq(0,0, *, *)\right\}=\left\{\mathcal{M} \mid \mu_{4} \in N \mathbb{Z} ;(e)\right.$ or $(f)$ or $(g)$ or $\left.(h)\right\}$ where $(e),(f),(g),(h)$ denote the following congruence conditions

(e) $v_{1}, w_{3} \equiv 0, d \neq \equiv(\bmod N)$,

(f) $c, w_{4}, v_{2}, d_{1} \equiv 0(\bmod N)$,

(g) $c \equiv 0, w_{3}, w_{4}, v_{1}, v_{2} \not \equiv 0(\bmod N)$,

(h) $w_{3}, d, c_{1} \equiv 0(\bmod N)$.

In case $(e)$ we set $c_{1} \equiv 1$ and $d_{1} \equiv v_{2} b \overline{v_{3} d}(\bmod N)$. For $(g)$ we assume for simplification that $\mu_{2} \in N \mathbb{Z}$ and choose

$$
d \equiv v_{2} w_{3} \overline{v_{1} w_{4}}, c_{1} \equiv 1 \text { and } d_{1} \equiv v_{2} b w_{4} \overline{v_{1} w_{3}}(\bmod N) .
$$

Proof. By Corollary 45 we need to determine congruence conditions for $M \in \mathcal{M} \iota_{1}(-J)$ such that $M \in \Gamma_{0}^{(2)}(N)$. Therefore, we use that

$$
\begin{aligned}
& \bigsqcup_{v, \sigma, \mu, w} P(N) L \gamma(v, \sigma)\left(\begin{array}{cc}
I_{2} & \mu \\
0 & I_{2}
\end{array}\right)\left(\begin{array}{ll}
w^{-1} & \\
& w^{T}
\end{array}\right) \iota_{1}(-J) \\
= & \bigsqcup_{v, \sigma, \mu, w} P(N) L\left(\begin{array}{cccc}
0 & v_{2} a & -v_{1} & v_{2} b \\
0 & v_{4} a & -v_{3} & v_{4} b \\
v_{4} & -v_{3} c & 0 & -v_{3} d \\
-v_{2} & v_{1} c & 0 & v_{1} d
\end{array}\right)\left(\begin{array}{cccc}
1 & & \\
-\mu_{2} & 1 & \mu_{4} \\
& & 1 & \mu_{2} \\
& & 1
\end{array}\right)\left(\begin{array}{cccc}
w_{1} & & \\
& w_{1} & -w_{3} & \\
& -w_{2} & w_{4} & \\
-w_{2} & & & w_{4}
\end{array}\right) .
\end{aligned}
$$

An element of this decomposition is in $\Gamma_{0}(N)$ if the lower right block matrix is congruent 0 modulo $N$. This is satisfied if

$$
\left(\begin{array}{cccc}
c_{1} & 0 & d_{1} & d_{2} \\
0 & 0 & 0 & 1
\end{array}\right)\left(\begin{array}{cc}
-v_{2} b w_{2}-v_{2} a\left(w_{1} \mu_{2}+w_{2} \mu_{4}\right)+\mu_{2} v_{1} w_{2} & v_{1} w_{2}+v_{2} a w_{1} \\
* & * \\
w_{1} v_{4}+v_{3} d w_{2}+\mu_{2} v_{3} c w_{1} & -v_{3} c w_{1} \\
-v_{2} w_{1}-v_{1} d w_{2}-\mu_{2} v_{1} c w_{1} & v_{1} c w_{1}
\end{array}\right) \equiv 0(\bmod N) .
$$


For simplicity, we assume that $\mu_{4} \in N \mathbb{Z}$. The lower right entry implies that $N \mid v_{1} c w_{1}$. If $N \mid v_{1}$, then the lower left entry implies that $N$ divides $w_{1}$. If

$$
c_{1} v_{2} b \equiv v_{3} d(\bmod N)
$$

the congruence is satisfied.

If $N \mid w_{1}$ and $N \nmid v_{1}$, then $N \mid d$ by the lower left entry. By the upper right entry, the congruence is only satisfied if either $N \mid v_{1}$ or $N \mid c_{1}$. If $N \mid v_{1}$, we obtain by the second lowest, left entry that $-c_{1} v_{2} b w_{2} \equiv 0(\bmod N)$, which can only be fulfilled if $N \mid c_{1}$.

Assume that $N \mid c$ and $N \nmid v_{1}, N \nmid w_{1}$. If $N \mid v_{2}$, then $N\left|w_{2}, N\right| d_{1}$ and the congruence is satisfied. If $N \nmid v_{2}$, then also $N \nmid w_{2}$. Hence, we require that $v_{2} w_{1} \equiv-v_{1} d w_{2}(\bmod N)$. Thus, the left entry in the third row is congruent to $d_{1} w_{1} v_{1}(\bmod N)$. If we assume that $\mu_{2}, \in N \mathbb{Z}$, it follows that

$$
c_{1} b v_{2} w_{2} \equiv d_{1} w_{1} v_{1}(\bmod N) \text {. }
$$

For the second decomposition, we proceed in the same way. We need to analyze under which condition

$$
\bigsqcup_{v, \sigma, \mu, w} P(N) L\left(\begin{array}{cccc}
v_{1} & v_{2} b & 0 & -v_{2} a \\
v_{3} & v_{4} b & 0 & -v_{4} a \\
0 & -v_{3} d & v_{4} & v_{3} c \\
0 & v_{1} d & -v_{2} & -v_{1} c
\end{array}\right)\left(\begin{array}{cccc}
1 & -\mu_{2} & \\
& 1 & & \\
& 1 & \\
-\mu_{4} & \mu_{2} & 1
\end{array}\right)\left(\begin{array}{cccc}
w_{4} & & -w_{2} \\
& w_{4} & -w_{2} & \\
& -w_{3} & w_{1} & \\
-w_{3} & & & w_{1}
\end{array}\right)
$$

is in $\Gamma_{0}^{(2)}(N)$. This is satisfied if

$$
\left(\begin{array}{cccc}
c_{1} & 0 & d_{1} & d_{2} \\
0 & 0 & 0 & \pm 1
\end{array}\right)\left(\begin{array}{cc}
v_{1} w_{4}+v_{2} a w_{3} & v_{2} b w_{4}-v_{1} \mu_{2} w_{4}+v_{2} a \mu_{2} w_{3} \\
* & * \\
-v_{3} c w_{3} & -v_{3} d w_{4}-v_{4} w_{3}-\mu_{2} v_{3} c w_{3} \\
v_{1} c w_{3} & v_{1} d w_{4}+v_{2} w_{3}+\mu_{2} v_{1} c w_{3}
\end{array}\right) \equiv 0(\bmod N)
$$

The case distinction works exactly as above.

\subsubsection{Bounds for Fourier coefficients of Klingen-Eisenstein series}

By applying the decompositions from the previous section, we now obtain explicit formulas for the Fourier coefficients of Klingen-Eisenstein series at $T>0$. For reasons of clarity, we put

$$
(f \|[M])(Z):=j(M, Z)^{-k} f(\pi(M Z)) .
$$

In Section 4.1 we already saw that

$$
(f \|[L M])(Z)=(f \mid[\omega(L)] \|[M])(Z)
$$


for $L \in P(\mathbb{Z})$ and $\omega(L)$ defined as in (71). Furthermore, we have

$$
\left(f \|\left[M\left(\begin{array}{cc}
I_{2} & \mu \\
0 & I_{2}
\end{array}\right)\left(\begin{array}{ll}
w^{-1} & \\
& w^{T}
\end{array}\right)\right]\right)(Z)=(f \|[M])\left(w^{-1} Z w^{T}+\mu\right)
$$

for $\mu \in \mathcal{P}(\mathbb{Z})$ and $w \in \mathrm{SL}_{2}(\mathbb{Z})$. A decisive role plays the following result [34, Lemma 15] from Kitaoka:

Lemma 49. Let $Z=\left(\begin{array}{ll}z_{1} & z_{2} \\ z_{2} & z_{4}\end{array}\right) \in \mathbb{H}_{2}, v=\left(\begin{array}{ll}v_{1} & v_{2} \\ v_{3} & v_{4}\end{array}\right)$ and $\sigma=\left(\begin{array}{ll}a & b \\ c & d\end{array}\right) \in \mathrm{SL}_{2}(\mathbb{Z})$ with $c v_{1} \neq 0$. Then, it holds that

$$
(f \|[\gamma(v, \sigma)])(Z)=j\left(\sigma, z_{4}\right)^{-k} f\left(z_{1} v_{1}^{2}-\left(z_{2} v_{1}-c^{-1} v_{2}\right)^{2}\left(z_{4}+c^{-1} d\right)^{-1}+a c^{-1} v_{2}^{2}\right) .
$$

Proof. Recall that $\gamma(v, \sigma)=\left(\begin{array}{ll}v & \\ & v^{-T}\end{array}\right) \iota_{2}(\sigma)$. Thus, we have

$$
j(\gamma(v, \sigma), Z)=\operatorname{det}\left(v^{-T}\left(\begin{array}{ll}
0 & \\
& c
\end{array}\right) Z+v^{-T}\left(\begin{array}{ll}
1 & \\
& d
\end{array}\right) Z\right)=j\left(\sigma, z_{4}\right)
$$

and

$$
\begin{aligned}
\gamma(v, \sigma)(Z) & =v\left(\left(\begin{array}{ll}
1 & \\
& a
\end{array}\right) Z+\left(\begin{array}{ll}
0 & \\
& b
\end{array}\right)\right)\left(\left(\begin{array}{ll}
0 & \\
& c
\end{array}\right) Z+\left(\begin{array}{ll}
1 & \\
& d
\end{array}\right)\right)^{-1} v^{T} \\
& =v\left(\begin{array}{cc}
z_{1}-c z_{2}^{2}\left(c z_{4}+d\right)^{-1} & z_{2}\left(c z_{4}+d\right)^{-1} \\
a z_{2}-c z_{2}\left(a z_{4}+b\right)\left(c z_{4}+d\right)^{-1} & \left(a z_{4}+b\right)\left(c z_{4}+d\right)^{-1}
\end{array}\right) v^{T} .
\end{aligned}
$$

Writing $b=c^{-1} a d-c^{-1}$ gives $(a z+b)(c z+d)^{-1}=a c^{-1}+c^{-1}\left(z_{4}+c^{-1} d\right)$. Hence,

$$
\begin{aligned}
\pi(\gamma(v, \sigma) Z) & =v_{1}^{2}\left(z_{1}-z_{2}^{2} c\left(c z_{4}+d\right)^{-1}\right)+2 v_{1} v_{2} z_{2}\left(c z_{4}+d\right)^{-1}+v_{2}^{2}\left(a c^{-1}-c^{-1}\left(c z_{4}+d\right)^{-1}\right) \\
& =v_{1}^{2} z_{1}-\left(v_{1} z_{2}-c^{-1} v_{2}\right)^{2}\left(z_{4}+c^{-1} d\right)^{-1}+v_{2}^{2} a c^{-1} .
\end{aligned}
$$

This proves the claim.

To evaluate the Fourier coefficients of $E(f, Z)=\sum_{T \in \mathcal{S}(Z)} A(T) e(\operatorname{tr} T Z)$ we express them as

$$
A(T)=e^{2 \pi \operatorname{tr}(T Y)} \int_{X \in \mathcal{P}(\mathbb{R}) / \mathcal{P}(\mathbb{Z})} \sum_{M \in P(N) \backslash \Gamma_{0}(N)}(f \|[M])(X+i Y) d X .
$$

To compute this integral, we apply the decomposition of $M \in P(N) \backslash \Gamma_{0}(N)$ with $\lambda(M) \neq(0,0, *, *)$ from the previous section step-by-step, following the approach in [34, Lemma 16]. 
Lemma 50. Let

$$
T=\left(\begin{array}{cc}
t_{1} & t_{2} / 2 \\
t_{2} / 2 & t_{4}
\end{array}\right) \in \mathscr{S}, \quad \sigma=\left(\begin{array}{cc}
a & * \\
c & d
\end{array}\right), v=\left(\begin{array}{cc}
v_{1} & v_{2} \\
* & *
\end{array}\right) \in \mathrm{SL}_{2}(\mathbb{Z})
$$

with $c>0, v_{1}>0$ and $f(z)=\sum_{n} a(n) e(n z) \in S_{k}(N)$. Then,

$$
\begin{aligned}
& \int_{X \in \mathcal{P}(\mathbb{R} / \mathbb{Z})} \sum_{\mu \in \mathcal{P}(\mathbb{Z}), \mu_{1}=0}\left(f \|\left[\gamma(v, \sigma)\left(\begin{array}{cc}
I & \mu \\
0 & I
\end{array}\right)\right]\right)(X+i Y) e(-\operatorname{tr}(T X)) d X \\
& =C_{k} e^{-2 \pi \operatorname{tr}(T Y)}(\operatorname{det} T)^{k-\frac{3}{2}} t_{1}^{1-k} c^{-k} a\left(t_{1} v_{1}^{-2}\right) e\left(\frac{t_{1} a v_{2}^{2} v_{1}^{-2}+t_{2} v_{2} v_{1}^{-1}+t_{4} d}{c}\right)
\end{aligned}
$$

with $d X=d x_{1} d x_{2} d x_{4}, v_{1}^{2} \mid t_{1}$ and

$$
C_{k}=\frac{(2 \pi)^{k-\frac{1}{2}}}{\Gamma\left(k-\frac{1}{2}\right) \sqrt{2} i^{k}} .
$$

Proof. By applying Lemma 49 the integral on the left hand equals

$$
\begin{gathered}
\int_{x_{1}, x_{2}, x_{4} \in \mathbb{R} / \mathbb{Z}} \sum_{\mu_{2}, \mu_{4} \in \mathbb{Z}}\left(f \|[\gamma(v, \sigma)]\left(\begin{array}{cc}
z_{1} & z_{2}+\mu_{2} \\
z_{2}+\mu_{2} & z_{4}+\mu_{4}
\end{array}\right)\right) e\left(-t_{1} x_{1}-t_{2} x_{2}-t_{4} x_{4}\right) d X \\
=\int_{\substack{x_{1} \in \mathbb{R} / \mathbb{Z} \\
x_{2}, x_{4} \in \mathbb{R}}}\left(c z_{4}+d\right)^{-k} f\left(z_{1} v_{1}^{2}-\left(z_{2} v_{1}-c^{-1} v_{2}\right)^{2}\left(z_{4}+c^{-1} d\right)^{-1}+a c^{-1} v_{2}^{2}\right) \\
\times e\left(-t_{1} x_{1}-t_{2} x_{2}-t_{4} x_{4}\right) d x_{1} d x_{2} d x_{4} .
\end{gathered}
$$

Taking the Fourier expansion of $f$ into account, the previous display equals

$$
\begin{aligned}
& =c^{-k} \sum_{n} a(n) e\left(n\left(i y_{1} v_{1}^{2}+a c^{-1} v_{2}^{2}\right) \int_{x_{1} \in \mathbb{R} / \mathbb{Z}} e\left(\left(n v_{1}^{2}-t_{1}\right) x_{1}\right) d x_{1}\right. \\
& \times \int_{x_{2}, x_{4} \in \mathbb{R}}\left(z_{4}+c^{-1} d\right)^{-k} e\left(n\left(-\left(z_{2} v_{1}-c^{-1} v_{2}\right)^{2}\left(z_{4}+c^{-1} d\right)^{-1}\right)-t_{2} x_{2}-t_{4} x_{4}\right) d x_{2} d x_{4} .
\end{aligned}
$$

The first integral vanishes unless $n=t_{1} v_{1}^{-2}$. In the second integral, we shift $x_{2}$ by $v_{1}^{-1} c^{-1} v_{2}$ and $x_{4}$ by $-c^{-1} d$. In this way, the previous display equals

$$
c^{-k} e^{-2 \pi t_{1} y_{1}} a\left(t_{1} v_{1}^{-2}\right) e\left(\frac{t_{1} a v_{2}^{2} v_{1}^{-2}-t_{2} v_{1}^{-1} v_{2}+t_{4} d}{c}\right) H(T, Y)
$$

where $H(T, Y)$ is given by

$$
\begin{aligned}
& \int_{x_{2}, x_{4} \in \mathbb{R}}\left(x_{4}+i y_{4}\right)^{-k} e\left(-t_{1}\left(x_{2}+i y_{2}\right)^{2}\left(x_{4}+i y_{4}\right)^{-1}-t_{2} x_{2}+t_{4} x_{4}\right) d x_{2} d x_{4} \\
& =\int_{x_{4} \in \mathbb{R}}\left(x_{4}+i y_{4}\right)^{-k} e\left(t_{1} y_{2}^{2}\left(x_{4}+i y_{4}\right)^{-1}-t_{4} x_{4}\right) \\
& \left.\times \int_{x_{2} \in \mathbb{R}} e\left(-t_{1}\left(x_{4}+i y_{4}\right)^{-1}\right) x_{2}^{2}-\left(2 t_{1} i y_{2}\left(x_{4}+i y_{4}\right)^{-1}+t_{2}\right) x_{2}\right) d x_{2} d x_{4}
\end{aligned}
$$


To compute this, we apply [23, 3.323/2]:

$$
\begin{aligned}
& =\left(2 i t_{1}\right)^{-\frac{1}{2}} e\left(i\left(t_{2}+t_{4}\right) y\right) \int_{x_{4} \in \mathbb{R}} x_{4}^{-k+1 / 2} e\left(-t_{4}+\left(t_{2} / 2\right)^{2} t_{1}^{-1} x_{4}\right) d x_{4} \\
& =e^{-2 \pi\left(t_{2}+t_{4}\right) y} i^{-k} 2^{-\frac{1}{2}}(2 \pi)^{k-\frac{1}{2}} \Gamma\left(k-\frac{1}{2}\right)^{-1} t_{1}^{1-k}(\operatorname{det} T)^{k-\frac{3}{2}}
\end{aligned}
$$

In the last step we used [23, 3.382/6].

Remark 51. To treat the term $L \in P(N) \backslash P(\mathbb{Z})$ in the decomposition of Lemma 46 we use 94). If $\omega(L) \notin \Gamma_{0}(N)$, then $f \mid[\omega(L)]$ is not a cusp form with respect to $\Gamma_{0}(N)$. In every case, $f \mid[\omega(L)]$ is a cusp form with respect to $\Gamma(N)$ and thus, we may expand

$$
f \mid[\omega(L)]=\sum_{n} b(n) e\left(\frac{n z}{N}\right)
$$

Using this Fourier expansion, we can proceed very similarly to the proof above. The key difference is that the $x_{1}$ integral vanishes unless $n=N t_{1} v_{1}^{-2}$. As a consequence, all the $N$ terms in the exponential sums cancel and we obtain with $\sigma, v$ and $T$ as above that:

$$
\begin{aligned}
& \int_{X \in \mathcal{P}(\mathbb{R} / \mathbb{Z})} \sum_{\mu \in \mathcal{P}(\mathbb{Z}), \mu_{1}=0}\left((f \mid[\omega(L)]) \|\left[\gamma(v, \sigma)\left(\begin{array}{cc}
I & \mu \\
0 & I
\end{array}\right)\right]\right)(X+i Y) e(-\operatorname{tr}(T X)) d X \\
& =C_{k} e^{-2 \pi \operatorname{tr}(T Y)}(\operatorname{det} T)^{k-\frac{3}{2}} t_{1}^{1-k} c^{-k} b\left(t_{1} N v_{1}^{-2}\right) e\left(\frac{t_{1} a v_{2}^{2} v_{1}^{-2}+t_{2} v_{2} v_{1}^{-1}+t_{4} d}{c}\right)
\end{aligned}
$$

with $v_{1}^{2} \mid N t_{1}$.

By Lemma 46 we now obtain a formula for the Fourier coefficients $A(T)$ of $E(f, Z)$ :

Lemma 52. Let

$$
f(z)=\sum_{n} a(n) e(n z) \text { and } N^{-k / 2}(f \mid[\eta])(z)=\sum_{n} b(n) e(z n) .
$$

Furthermore, let

$$
T[w]=w^{T} T w=\left(\begin{array}{ll}
T[w]_{1} & T[w]_{2} \\
T[w]_{2} & T[w]_{4}
\end{array}\right) \in \mathscr{S}
$$


and $C_{k}$ be given by 95 . Then,

$$
\begin{aligned}
A(T) & =e^{2 \pi \operatorname{tr}(T Y)} \int_{X \in \mathcal{P}(\mathbb{R} / \mathbb{Z})} \sum_{\substack{M \in P(N) \backslash \Gamma_{0}(N) \\
\lambda(M) \neq(0,0, *, *)}}\left(f||[M]_{k}\right)(Z) e(-\operatorname{tr}(T X)) d X \\
& =C_{k}(\operatorname{det} T)^{k-\frac{3}{2}} \sum_{\substack{w_{3} \geqslant 0 \\
\left(w_{1}, w_{3}\right)=1}}\left(T[w]_{1}\right)^{1-k}\left(\sum_{c>0, N \mid c} c^{-k} \sum_{\substack{v_{1}>0 \\
v_{1}^{2} \mid T[w]_{1}}} a\left(\frac{T[w]_{1}}{v_{1}^{2}}\right) K\left(v_{1}, c, T[w]\right)\right. \\
& \left.+\sum_{c>0, N \nmid c} c^{-k} \sum_{\substack{v_{1}>0, N\left|v_{1} \\
v_{1}^{2}\right| N T[w]_{1}}} b\left(\frac{T[w]_{1} N}{v_{1}^{2}}\right) \tilde{K}\left(v_{1}, c, T[w]\right)\right),
\end{aligned}
$$

where

$$
K\left(v_{1}, c, T[w]\right)=\sum_{\substack{d \neq 0 \bmod c \\ a d \equiv=1 \bmod c\left(v_{2} \bmod c v_{1} \\\left(v_{1}, v_{2}\right)=1\right.}} e\left(\frac{T[w]_{1} v_{1}^{-2} a v_{2}^{2}+T[w]_{2} v_{1}^{-1} v_{2}+T[w]_{4} d}{c}\right)
$$

and $\tilde{K}\left(N v_{1}, c, T[w]\right)$ equals

$\sum_{\substack{d \neq 0 \bmod c \\ a d \equiv 1 \bmod c}} \sum_{\substack{v_{2} \bmod c N v_{1} \\\left(N v_{1}, v_{2}\right)=1}} e\left(\frac{\tilde{T}[w]_{1} v_{1}^{-2} a v_{2}^{2}+T[w]_{2} v_{1}^{-1} v_{2}}{N c}\right) e\left(-\frac{\tilde{T}[w]_{1} v_{1}^{-2} a v_{2}^{2} \bar{c}}{N}\right) e\left(\frac{T[w]_{4} d}{c}\right)$

where $\tilde{T}[w]_{1}=\frac{T[w]_{1}}{N}$ and $\bar{c} c \equiv 1 \bmod N$.

Proof. By Lemma 46 and $(94)$ the integral equals

$\int_{X \in \mathcal{P}(\mathbb{R} / \mathbb{Z})} \sum_{\tau, \sigma, \mu, v, w}\left((f[\omega(L)]) \|\left[\gamma(v, \sigma)\left(\begin{array}{cc}I & \mu \\ & I\end{array}\right)\left(\begin{array}{cc}w^{-1} & \\ & w^{T}\end{array}\right)\right]\right)(Z) e(-\operatorname{tr}(T X)) d X$

with $L, \sigma, \lambda, v, w$ running over the cosets from Lemma 46 . We substitute $X$ by $w X w^{T}$ getting

$\int_{X \in \mathcal{P}(\mathbb{R} / \mathbb{Z})} \sum_{\tau, \sigma, \mu, v, w}((f \mid[\omega(L)]) \|[\gamma(v, \sigma)])\left(X+\mu+w^{-1} Y w^{-T}\right) e\left(-\operatorname{tr}\left(w^{T} T w X\right)\right) d X$.

Moreover, note that

$$
\sigma=\left(\begin{array}{ll}
* & * \\
c & d
\end{array}\right) \in \Gamma_{\infty} \backslash \mathrm{SL}_{2}(\mathbb{Z}) / \Gamma_{\infty}, c>0
$$

translates into $c>0$ and $d \bmod c$ with $(d, c)=1$ as well as

$$
v=\left(\begin{array}{cc}
v_{1} & v_{2} \\
* & *
\end{array}\right) \in \Gamma_{\infty}^{T} \backslash \mathrm{SL}_{2}(\mathbb{Z}) / \Gamma_{\infty}(c), v_{1}>0
$$


corresponds to $v_{1}>0$ and $v_{2} \bmod c v_{1}$ with $\left(v_{1}, v_{2}\right)=1$. Furthermore

$$
w=\left(\begin{array}{ll}
w_{1} & * \\
w_{3} & *
\end{array}\right) \in \operatorname{PSL}_{2}(\mathbb{Z}) / \bar{\Gamma}_{\infty}
$$

is equivalent to $w_{3} \geqslant 0$ and $w_{1} \in \mathbb{Z}$ with $\left(w_{1}, w_{3}\right)=1$.

For the case $N \mid v_{1}$, we use that $J\left(\begin{array}{cc}N & \\ & N\end{array}\right)=\eta\left(\begin{array}{ll}1 & \\ & N\end{array}\right)$ and obtain

$$
f|[\omega(L)]=f|\left[J\left(\begin{array}{cc}
1 & d_{1} \\
& 1
\end{array}\right)\right]=f \mid\left[\eta\left(\begin{array}{cc}
1 & d_{1} \\
& N
\end{array}\right)\right]=\sum_{n} b(n) e\left(\frac{n d_{1}}{N}\right) e\left(\frac{n z}{N}\right)
$$

where $d_{1} \equiv a v_{2}^{2} \bar{c}(\bmod N)$ and $b(n)$ denotes the Fourier coefficients of $N^{-\frac{k}{2}} f \mid[\eta]$. The rest follows by Lemma 50 and Remark 51 .

For the second Klingen-Eisenstein series $E_{N}(f, Z)=\sum_{T} B(T) e(\operatorname{tr}(T Z))$ we proceed similarly:

Lemma 53. Let

$$
T[w]=w^{T} T w=\left(\begin{array}{ll}
T[w]_{1} & T[w]_{2} \\
T[w]_{2} & T[w]_{4}
\end{array}\right) \in \mathscr{S}
$$

and $\left.N^{-k / 2} f \mid[\eta]\right)(z)=\sum_{n} b(n) e(n z) \in S_{k}(N)$. Then,

$$
\begin{aligned}
& B(T)=C_{k}(\operatorname{det} T)^{k-\frac{3}{2}} \sum_{v_{1}>0} \sum_{\substack{\left(w_{1}, w_{3}\right)=1, w_{3} \geqslant 0 \\
v_{1}^{2} \mid T[w]_{1}}}\left(T[w]_{1}\right)^{1-k} a\left(\frac{T[w]_{1}}{v_{1}^{2}}\right) \sum_{c>0} c^{-k} \\
& \times \sum_{\substack{d \neq 0 \bmod N c \\
N \mid d, a d \equiv 1 \bmod c}} \sum_{\substack{v_{2} \bmod N c v_{1} \\
N \mid v_{2},\left(v_{1}, v_{2}\right)=1}} e\left(\frac{T[w]_{1} a v_{2}^{2} v_{1}^{-2}+T[w]_{2} v_{2} v_{1}^{-1}+T[w]_{4} d}{N c}\right) .
\end{aligned}
$$

Proof. We use

$$
B(T)=N^{k} \int_{X \in \mathcal{P}(\mathbb{R}) / \mathcal{P}(\mathbb{Z})} \sum_{\substack{M \in P(N) \backslash \Gamma_{0}^{(2)}(N) J_{4} \\ \lambda(M) \neq(0,0, *, *)}}(f \| M)(N Z) e(-\operatorname{tr} T X) d X .
$$

By Lemma 47 this equals

$$
N^{k} \int_{X \in \mathcal{P}(\mathbb{R}) / \mathcal{P}(\mathbb{Z})} \sum_{v, \sigma, w, \mu}((f \mid[J]) \|[\gamma(v, \sigma)])\left(N w^{-1} Z w^{-T}+\mu\right) e(-\operatorname{tr} T X) d X
$$


with $\mu_{2}, \mu_{4} \in N \mathbb{Z}$ and $\sigma, v, w$ as in Lemma 44 (with $N_{1}=N_{2}=N$ ), $N \mid d$ and $N \mid v_{2}$. This can be computed in the same way as in Lemma 50. The first step is to substitute $X$ by $w X w^{T}$ and apply Lemma 49 . This gives

$$
\begin{aligned}
B(T) & =N^{k} \sum_{c, d, w_{1}, w_{3}, v_{1}, v_{2}} \int_{\substack{x_{1} \in \mathbb{R} / \mathbb{Z} \\
x_{2}, x_{4} \in \mathbb{R}}}\left(N\left(x_{4}+\tilde{y}_{4}+\frac{d}{N c}\right)\right)^{-k} e\left(-T[w]_{1} x_{1}-T[w]_{2} x_{2}-T[w]_{4} x_{4}\right) \\
& \times(f \mid[J])\left(N\left(\left(x_{1}+i \tilde{y}_{1}\right) v_{1}^{2}-\left(\left(x_{2}+i \tilde{y}_{2}\right) v_{1}-\frac{v_{2}}{N c}\right)^{2}\left(x_{4}+i \tilde{y}_{4}+\frac{d}{N c}\right)^{-1}+\frac{a v_{2}^{2}}{N c}\right)\right) d X \\
& =\sum_{c, d, w_{1}, w_{3}, v_{1}, v_{2}} c^{-k} \sum_{n} b(n) e\left(n\left(i \tilde{y}_{1} v_{1}^{2}+\frac{a v_{2}^{2}}{N c}\right)\right) \int_{x_{1} \in \mathbb{R} / \mathbb{Z}} e\left(\left(n v_{1}^{2}-T[w]_{1}\right) x_{1}\right) d x_{1} \\
& \times \int_{x_{2}, x_{4} \in \mathbb{R}}\left(x_{4}+i \tilde{y}_{4}+\frac{d}{N c}\right)^{-k} e\left(-T[w]_{2} x_{2}-T[w]_{4} x_{4}\right) \\
& \times e\left(n\left(-\left(x_{2}+i \tilde{y}_{2}\right) v_{1}-\frac{v_{2}}{N c}\right)^{2}\left(x_{4}+i \tilde{y}_{4}+\frac{d}{N c}\right)\right) d x_{2} d x_{4},
\end{aligned}
$$

where $(f \mid[J])(z)=\sum_{n} b(n) e\left(\frac{n z}{N}\right), \tilde{y}_{1}, \tilde{y}_{2}, \tilde{y}_{4}$ and $T[w]_{1}, T[w]_{2}, T[w]_{4}$ are the entries of $w^{-1} Y w^{-T}$ and $w^{T} T w$ respectively. The rest of the proof works exactly as in Lemma 50 and 52 ,

By means of these explicit formulas, we can bound the Fourier coefficients $A(T)$ of $E\left(f_{1}, Z\right)$ and $B(T)$ of $E_{N}\left(f_{2}, Z\right)$ in the asymptotic formula of $r(Q, T)$. For this purpose, we start with two preparatory lemmas:

Lemma 54. Let $K\left(v_{1}, c, T[w]\right)$ be defined as in Lemma 52 with $v_{1}^{2} \mid T[w]_{1}$. Then,

$$
K\left(v_{1}, N c, T[w]\right) \ll N^{\frac{3}{2}}(N, T[w])^{\frac{1}{2}} c^{2} v_{1}(N c)^{\epsilon} .
$$

Moreover, for $\tilde{K}\left(v_{1}, c, T[w]\right)$ as in Lemma 52 with $v_{1}^{2} \mid N T[w]_{1}$ and $N^{r}:=\left(v, N^{\infty}\right)$, it holds that

$$
\tilde{K}\left(v_{1}, c, T[w]\right) \leqslant\left(N^{r}, T[w]_{2}\right) \frac{v_{1}}{N^{r}} c .
$$

Proof. We write

$$
K\left(v_{1}, N c, T[w]\right)=\sum_{\substack{v_{2}\left(\bmod N c v_{1}\right) \\\left(v_{1}, v_{2}\right)=1}} e\left(\frac{T[w]_{2} v_{2}}{N c v_{1}}\right) \sum_{\substack{d \neq 0(\bmod N c) \\ a d \equiv 1 \bmod c}} e\left(\frac{T[w]_{1} v_{1}^{-2} v_{2}^{2} a+T[w]_{4} d}{N c}\right) .
$$

We decompose the first sum according to whether $N \mid v_{2}$. If $N \mid v_{2}$, we trivially estimate $K\left(v_{1}, N c, T[w]\right) \leqslant N v_{1} c^{2}$. If $N \nmid v_{2}$, the Weil bound for Kloosterman sums gives

$$
\sum_{d(\bmod N c)}^{*} e\left(\frac{T[w]_{1} v_{1}^{-2} v_{2}^{2} \bar{d}+T[w]_{4} d}{N c}\right) \ll c N^{1 / 2}\left(N, T[w]_{1} v_{1}^{-2}, T[w]_{4}\right)^{1 / 2}(N c)^{\epsilon} .
$$


If $N \nmid T[w]_{1} v_{1}^{-2}$ or $N \nmid T[w]_{4}$ the greatest common divisor above equals 1 . If $N \mid T[w] v_{1}^{-2}$ the Gauss sum

$$
\sum_{v_{2}\left(\bmod N c v_{1}\right)} e\left(\frac{T[w]_{1} v_{1}^{-1} v_{2}^{2} a+T[w]_{2} v_{2}}{N c v_{1}}\right)
$$

vanishes unless $N \mid T[w]_{2}$. Thus, if one of the entries of $2 T[w]$ is not divisible by $N$, we save a factor of $N^{\frac{1}{2}}$ compared to trivial estimation.

Let $v_{1}=n^{r} \tilde{v}_{1}$ with $\left(N, \tilde{v}_{1}\right)=1$. It follows that $N^{2 r-1} \mid T[w]_{1}$. We decompose the $v_{2}$ sum into a term of modulus $N^{r}$ and one of $\tilde{v}_{1} c$. We estimate the latter sum trivially by $\tilde{v}_{1} c$. The former sum equals

$$
\sum_{v_{2}\left(\bmod N^{r}\right)}^{*} e\left(\frac{T[w]_{1} N^{-2 r+1} a v_{2}^{2} \bar{c}-T[w]_{1} N^{-2 r+1} a v_{2}^{2} \bar{c}}{N}\right) e\left(\frac{T[w]_{2} v_{2}}{N^{r}}\right) .
$$

The first term cancels. The Ramanujan sum equals

$$
\mu\left(\frac{N^{r}}{\left(N^{r}, T[w]_{2}\right)}\right) \frac{\varphi\left(N^{r}\right)}{\varphi\left(\frac{N^{r}}{\left(N^{r}, T[w]_{2}\right)}\right)} \leqslant\left(N^{r}, T[w]_{2}\right) .
$$

Lemma 55. Let $T=\left(\begin{array}{cc}t_{1} & t_{2} / 2 \\ t_{2} / 2 & t_{4}\end{array}\right) \in \mathscr{S}$ be Minkowski-reduced and $r \geqslant 1$. Then,

$$
\sum_{\left(w_{1}, w_{3}\right)=1, w_{3} \geqslant 1}\left(w_{1}^{2} t_{1}+w_{1} w_{3} t_{2}+w_{3}^{2} t_{4}\right)^{-r} \ll t_{1}^{-\frac{1}{2}} t_{4}^{\frac{1}{2}-r} \ll(\operatorname{det} T)^{-\frac{r}{2}} .
$$

Proof. We estimate

$$
\sum_{\left(w_{1}, w_{3}\right)=1, w_{3} \geqslant 1}\left(w_{1}^{2} t_{1}+w_{1} w_{3} t_{2}+w_{3}^{2} t_{4}\right)^{-r} \ll t_{4}^{-r} \sum_{w_{1} \geqslant 0, w_{3} \geqslant 1}\left(w_{1}^{2} \frac{t_{1}}{t_{4}}+w_{3}^{2}\right)^{-r} .
$$

To estimate the latter term we set $t=\frac{t_{4}}{t_{1}} \geqslant 1$ and conclude the argument by

$$
\begin{array}{r}
\sum_{w_{1} \geqslant 0, w_{3} \geqslant 1}\left(w_{1}^{2} t^{-1}+w_{3}^{2}\right)^{-r}=\sum_{w_{3} \geqslant 1} \sum_{n=0}^{\infty} \sum_{n \sqrt{t} \leqslant \frac{w_{1}}{w_{3}} \leqslant(n+1) \sqrt{t}}\left(w_{1}^{2} t^{-1}+w_{3}^{2}\right)^{-r} \\
\leqslant \sqrt{t} \sum_{n=0}^{\infty} \sum_{w_{3} \geqslant 1}\left((n+1) w_{3}\right)^{-2 r} \ll \sqrt{t} .
\end{array}
$$


If $q(x)=\frac{1}{2} x^{T} Q x=\sum_{i} a_{i} x_{i}^{2}$ with $a_{1} \leqslant \ldots \leqslant a_{2 k}$ we set $a=a_{2 k} a_{2 k-1}$ and $\tilde{a}=a_{1} a_{2}$. Otherwise, we set $a=\tilde{a}=(\operatorname{det} Q)^{\frac{1}{k}}$. As a consequence of Lemma 52 , 53 . 54 and 55, we obtain the following bound for the Fourier coefficients of the Klingen-Eisenstein series:

Theorem 56. For $T>0$, set

$$
\min T=\min _{0 \neq x \in \mathbb{Z}^{2}} x^{T} T x
$$

Then, it holds for the Fourier coefficients $A(T)$ of $E\left(f_{1}, Z\right)$ that

$$
\begin{aligned}
A(T) & \ll(\operatorname{det} T)^{k-\frac{3}{2}}(\min T)^{\frac{1-k}{2}}\left(\left(1+\frac{(\min T)^{\frac{1}{4}}}{N^{\frac{1}{2}}}\right) \frac{N^{\frac{1-k}{2}+\epsilon} \sqrt[4]{\tilde{a}}}{\sqrt{\operatorname{det} Q}}(N, \operatorname{det} T) \delta_{N \mid \min T}\right. \\
& \left.+(N, T)^{\frac{1}{2}}\left(1+\frac{(\min T)^{\frac{1}{4}}(\min T, N)^{\frac{1}{4}}}{N^{\frac{1}{2}}}\right)\left(\frac{N^{\frac{3-k}{2}}}{\sqrt{\operatorname{det} Q}}+\frac{N^{-k+2}}{\sqrt[4]{a}}\right) N^{\epsilon}\right) \\
& +(\operatorname{det} T)^{\frac{3}{4} k-\frac{5}{4}}\left(\frac{N^{2-\frac{k}{2}}}{\sqrt{\operatorname{det} Q}}+\frac{N^{-k+\frac{5}{2}}}{\sqrt[4]{a}}\right)\left(1+\frac{(\operatorname{det} T)^{\frac{1}{8}}}{N^{\frac{1}{4}}}\right) N^{\epsilon}
\end{aligned}
$$

where $(N, T)=\left(N, t_{1}, t_{2}, t_{4}\right)$ for $T=\left(\begin{array}{cc}t_{1} & t_{2} / 2 \\ t_{2} / 2 & t_{4}\end{array}\right)$. For the Fourier coefficients $B(T)$ of $E_{N}\left(f_{2}, Z\right)$, we have that

$$
\begin{aligned}
B(T) & \ll(\operatorname{det} T)^{k-\frac{3}{2}}\left(\frac{N^{\frac{k}{2}}}{\operatorname{det} Q}+\frac{N^{\frac{1}{2}}}{\sqrt{\operatorname{det} Q} \sqrt[4]{a}}\right) N^{\epsilon} \\
& \times\left((\min T)^{\frac{1-k}{2}}\left(1+\frac{(\min T)^{\frac{1}{4}}(\min T, N)^{\frac{1}{4}}}{N^{\frac{1}{2}}}\right)+(\operatorname{det} T)^{\frac{1-k}{4}}\left(1+\frac{(\operatorname{det} T)^{\frac{1}{8}}}{N^{\frac{1}{4}}}\right)\right) .
\end{aligned}
$$

Proof. We apply the formula of Lemma 52 and decompose $A(T)=A_{c}(T)+A_{v}(T)$ according to whether $N \mid c$ or $N \mid v_{1}$ and $N \nmid c$. For the former term we have by Lemma 52 that

$$
\begin{aligned}
A_{c}(T) & \ll(\operatorname{det} T)^{k-\frac{3}{2}} \sum_{w \in \mathrm{SL}_{2}(\mathbb{Z}) / \Gamma_{\infty}}\left(T[w]_{1}\right)^{1-k} \sum_{c \geqslant 1}(N c)^{-k} \\
& \times \sum_{v_{1} \geqslant 1, v_{1}^{2} \mid T[w]_{1}}\left|a\left(\frac{T[w]_{1}}{v_{1}^{2}}\right)\right|\left|K\left(v_{1}, N c, T[w]\right)\right| .
\end{aligned}
$$

Recall that $a(n)$ denote the Fourier coefficients of $f_{1}(z)=\theta(Q, z)-\theta($ gen $Q, z)$ and $T[w]=w^{t} T w$. By Lemma 54 , we have that $K\left(v_{1}, N c, T[w]\right) \ll N^{\frac{3}{2}}(N, T[w])^{\frac{1}{2}} c^{2} v_{1}$. For the Fourier coefficients, we apply Cauchy-Schwarz and the Petersson formula, cf. [27, Corollary 14.24]. This gives

$$
a\left(\frac{T[w]_{1}}{v_{1}^{2}}\right) \ll\left\|f_{1}\right\|\left(\frac{T[w]_{1}}{v_{1}^{2}}\right)^{\frac{k-1}{2}+\epsilon}\left(1+\frac{T[w]_{1}^{\frac{1}{4}}\left(T[w]_{1} v_{1}^{-2}, N\right)^{\frac{1}{4}}}{v_{1}^{\frac{1}{2}} N^{\frac{1}{2}}}\right) N^{\epsilon}
$$


Furthermore, it holds by Lemma 24 that

$$
\left\|f_{1}\right\| \ll \frac{N^{\frac{k}{2}+\epsilon}}{\sqrt{\operatorname{det} Q}}+\frac{N^{\frac{1}{2}+\epsilon}}{\sqrt[4]{a}} .
$$

Since $A(T)=A\left(U^{T} T U\right)$ for $U \in \mathrm{GL}_{2}(\mathbb{Z})$, we may assume that $T=\left(\begin{array}{cc}t_{1} & t_{2} / 2 \\ t_{2} / 2 & t_{4}\end{array}\right)$ is Minkowski-reduced, which means that $t_{1}=\min T, t_{2} \leqslant t_{1}$ and $t_{1} t_{4}=\operatorname{det} T$. If $w_{3}=0$ this implies that $w_{1}=1$ and thus, $w^{T} T w=T$. We infer that

$$
\begin{aligned}
A_{c}(T) & \ll(\operatorname{det} T)^{k-\frac{3}{2}} N^{\frac{3}{2}-k}\left(\frac{N^{\frac{k}{2}+\epsilon}}{\sqrt{\operatorname{det} Q}}+\frac{N^{\frac{1}{2}+\epsilon}}{\sqrt[4]{a}}\right) \sum_{c \geqslant 1} c^{2-k} \\
& \times\left(\sum_{v_{1} \geqslant 1, v_{1}^{2} \mid t_{1}} v_{1}^{2-k} t_{1}^{\frac{1-k}{2}+\epsilon}(N, T)^{\frac{1}{2}}\left(1+\frac{t_{1}^{\frac{1}{4}}\left(N, t_{1}\right)^{\frac{1}{4}}}{v_{1}^{\frac{1}{2}} N^{\frac{1}{2}}}\right)\right. \\
& \left.+\sum_{\substack{\left(w_{1}, w_{3}\right)=1 \\
w_{3}>0}}\left(T[w]_{1}\right)^{\frac{1-k}{2}+\epsilon}(N, T[w])^{\frac{1}{2}} \sum_{\substack{v_{1} \geqslant 1 \\
v_{1}^{2} \mid T[w]_{1}}} v_{1}^{2-k}\left(1+\frac{T[w]_{1}^{\frac{1}{4}}\left(N, T[w]_{1}\right)^{\frac{1}{4}}}{v_{1}^{\frac{1}{2}} N^{\frac{1}{2}}}\right)\right) .
\end{aligned}
$$

For the $w_{1}, w_{3}$ sum, we recall that $T[w]_{1}=w_{1}^{2} t_{1}+w_{1} w_{3} t_{2}+w_{3}^{2} t_{4}$ and apply Lemma 55 which states that

$$
\sum_{\left(w_{1}, w_{3}\right)=1, w_{3}>0}\left(T[w]_{1}\right)^{-r} \ll(\operatorname{det} T)^{-\frac{r}{2}}
$$

Furthermore, we trivially estimate $(N, T[w]) \leqslant N$ and $\left(N, T[w]_{1}\right) \leqslant N$.

To treat $A_{v}(T)$ we proceed similarly. In this case, we need to estimate the Fourier coefficients of

$$
N^{-k / 2} f_{1} \mid[\eta](z)=(\operatorname{det} Q)^{-1 / 2}\left(\theta\left(N Q^{-1}, z\right)-\theta\left(\operatorname{gen} N Q^{-1}, z\right)\right)=\sum_{n} b(n) e(n z) .
$$

By Lemma 24 and the Petersson formula we obtain that

$$
b\left(\frac{T[w]_{1}}{N v_{1}^{2}}\right) \ll\left(N^{-\frac{k}{2}}+\frac{\sqrt[4]{\tilde{a}}}{\sqrt{\operatorname{det} Q}}\right)\left(\frac{T[w]_{1}}{N v_{1}^{2}}\right)^{\frac{k-1}{2}+\epsilon}\left(1+\frac{T[w]_{1}^{\frac{1}{4}}}{v_{1}^{\frac{1}{2}} N^{\frac{1}{2}}}\right) N^{\epsilon} .
$$

For simplicity, we estimated $\left(\frac{T[w]_{1}}{v_{1}^{2} N}, N\right)^{1 / 4} \leqslant N^{1 / 4}$. Furthermore, by Lemma 54 it holds that $K\left(N v_{1}, c, T[w]\right) \leqslant\left(N, T[w]_{2}\right) v_{1} c^{2}$. For $T \in \mathcal{R}_{2}$, i.e. Minkowski-reduced, 
we infer

$$
\begin{aligned}
A_{v}(T) & \ll(\operatorname{det} T)^{k-\frac{3}{2}} N^{\frac{1-k}{2}+\epsilon}\left(N^{-k+\frac{1}{2}}+\frac{N^{\frac{1-k}{2}} \sqrt[4]{\tilde{a}}}{\sqrt{\operatorname{det} Q}}\right) \sum_{N \nmid c} c^{2-k} \\
& \times\left(\left(N, t_{2}\right) \sum_{\substack{v_{1} \geqslant 1 \\
v_{1}^{2} N \mid t_{1}}} v_{1}^{2-k} t_{1}^{\frac{1-k}{2}+\epsilon}\left(1+\frac{t_{1}^{\frac{1}{4}}}{v_{1}^{\frac{1}{2}} N^{\frac{1}{2}}}\right)\right. \\
& \left.+N \sum_{\substack{\left(w_{1}, w_{3}\right)=1 \\
w_{3} \neq 0}}\left(T[w]_{1}\right)^{\frac{1-k}{2}+\epsilon} \sum_{\substack{v_{1} \geqslant 1 \\
v_{1}^{2} N \mid T[w]_{1}}} v_{1}^{2-k}\left(1+\frac{T[w]_{1}^{\frac{1}{4}}}{v_{1}^{\frac{1}{2}} N^{\frac{1}{2}}}\right)\right) .
\end{aligned}
$$

Note that $N \mid t_{1}$ implies that $\left(N, t_{2}\right)=(N$, $\operatorname{det} T)$. As last step, we apply Lemma 55 to evaluate the $w_{1}, w_{3}$ sum.

To estimate $B(T)$ we use the formula from Lemma 53. We substitute $d$ by $N d$ and $v_{2}$ by $N v_{2}$ and estimate the exponential sum

$$
\sum_{\substack{d \neq 0 \bmod c \\ a N d \equiv 1 \bmod c}} \sum_{\substack{v_{2} \bmod c v_{1} \\\left(v_{1}, N v_{2}\right)=1}} e\left(\frac{T[w]_{1} a v_{2}^{2} N v_{1}^{-2}+T[w]_{2} v_{2} v_{1}^{-1}+T[w]_{4} d}{c}\right)
$$

trivially by $c^{2} v_{1}$. Recall that $f_{2}(z)=\frac{N^{k}}{\operatorname{det} Q}\left(\theta\left(N Q^{-1}, z\right)-\theta\left(\right.\right.$ gen $\left.\left.N Q^{-1}, z\right)\right)$. Hence,

$$
N^{-k / 2} f_{2} \mid[\eta]=(\operatorname{det} Q)^{-\frac{1}{2}}(\theta(Q, z)-\theta(\operatorname{gen} Q, z)) .
$$

Proceeding similarly to the case of $A_{c}(T)$ we obtain for $T \in \mathcal{R}_{2}$ that

$$
\begin{aligned}
B(T) & \ll(\operatorname{det} T)^{k-\frac{3}{2}}\left(\frac{N^{\frac{k}{2}+\epsilon}}{\operatorname{det} Q}+\frac{N^{\frac{1}{2}+\epsilon}}{\sqrt{\operatorname{det} Q} \sqrt[4]{a}}\right) \sum_{c \geqslant 1} c^{2-k} \\
& \times\left(\sum_{v_{1} \geqslant 1, v_{1}^{2} \mid t_{1}} v_{1}^{2-k} t_{1}^{\frac{1-k}{2}+\epsilon}\left(N, t_{1}\right)^{\frac{1}{2}}\left(1+\frac{t_{1}^{\frac{1}{4}}\left(N, t_{1}\right)^{\frac{1}{4}}}{v_{1}^{\frac{1}{2}} N^{\frac{1}{2}}}\right)\right. \\
& \left.+\sum_{\left(w_{1}, w_{3}\right)=1, w_{3} \neq 0}\left(T[w]_{1}\right)^{\frac{1-k}{2}+\epsilon} N^{\frac{1}{2}} \sum_{\substack{v_{1} \geqslant 1 \\
v_{1}^{2} \mid T[w]_{1}}} v_{1}^{2-k}\left(1+\frac{T[w]_{1}^{\frac{1}{4}} N^{\frac{1}{4}}}{v_{1}^{\frac{1}{2}} N^{\frac{1}{2}}}\right)\right) .
\end{aligned}
$$

By applying Lemma 55 the claim follows.

It remains to estimate the contribution of Klingen-Eisenstein series to the inner product. Therefore, we treat each coset class from Lemma 36 separately. We start with easiest class $R(0)$ that only contains the identity. By Lemma 37 we have a contribution of size

$$
\sum_{T>0, t_{1},\left|t_{2}\right|, t_{4} \ll 1}|A(T)|^{2}+|B(T)|^{2} \ll \frac{N^{\frac{k}{2}}}{\operatorname{det} Q}+\frac{N^{\frac{1}{2}}}{\sqrt{\operatorname{det} Q} \sqrt[4]{a}} .
$$


The treatment of the $R(3)$ class is already more complex. We use the following trick, to obtain bounds for the Fourier coefficients of $E\left(f_{1}, Z\right) \mid\left[J_{4}\right]$ and $E_{N}\left(f_{2}, Z\right) \mid\left[J_{4}\right]$ :

$$
\begin{gathered}
E\left(f_{1}, Z\right)\left|\left[J_{4}\right]=E\left(f_{1}, Z\right)\right|\left[J_{4}\left(\begin{array}{ll}
N I & \\
& N I
\end{array}\right)\right]=E_{N}\left(f_{1}, Z\right) \mid\left[\left(\begin{array}{ll}
I & \\
& N I
\end{array}\right)\right], \\
E_{N}\left(f_{2}, Z\right)\left|\left[J_{4}\right]=E\left(f_{2}, Z\right)\right|\left[\left(\begin{array}{ll}
N I & -I
\end{array}\right) J_{4}\right]=E\left(f_{2}, Z\right) \mid\left[\left(\begin{array}{ll}
I & \\
& N I
\end{array}\right)\right] .
\end{gathered}
$$

As a consequence, we obtain

$$
\begin{aligned}
E\left(f_{1}, Z\right) \mid\left[J_{4}\right] & =\sum_{T \in \mathcal{S}} A_{3}(T) e\left(\frac{\operatorname{tr}(T Z)}{N}\right), \\
E_{N}\left(f_{2}, Z\right) \mid\left[J_{4}\right] & =\sum_{T \in \mathcal{S}} B_{3}(T) e\left(\frac{\operatorname{tr}(T Z)}{N}\right),
\end{aligned}
$$

where $A_{3}(T), B_{3}(T)$ are the Fourier coefficients of $N^{-k} E_{N}\left(f_{1}, Z\right)$ and $N^{-k} E\left(f_{2}, Z\right)$ respectively. By applying Theorem 56 we obtain the bounds

$$
\begin{aligned}
& A_{3}(T) \ll(\operatorname{det} T)^{k-\frac{3}{2}}(\min T)^{\frac{1-k}{2}} N^{-k}\left(N^{-\frac{k}{2}}+\frac{\sqrt[4]{\tilde{a}}}{\sqrt{\operatorname{det} Q}}\right), \\
& B_{3}(T) \ll\left((\operatorname{det} T)^{k-\frac{3}{2}}(\min T)^{\frac{1-k}{2}} \sqrt{(N, T)}+\operatorname{det} T^{-\frac{3}{4} k+\frac{5}{4}} \sqrt{N}\right) N^{-k}\left(\frac{N^{\frac{3-k}{2}}}{\sqrt{\operatorname{det} Q}}+\frac{N^{\frac{3}{2}} \sqrt[4]{\tilde{a}}}{\operatorname{det} Q}\right) .
\end{aligned}
$$

By Lemma 38 and assuming that $T \in \mathcal{R}_{2}$ it follows that the contribution in the $R(3)$ case is bounded by

$$
N^{4 k-3} \sum_{\substack{t_{1}, t_{2} t_{4} \ll N^{2+\epsilon} \\\left|t_{2}\right| \leqslant t_{1}}} \frac{A_{3}(T)^{2}+B_{3}(T)^{2}}{(\operatorname{det} T)^{2 k-3}} \ll \frac{N^{2 k+2} \sqrt{\tilde{a}}}{(\operatorname{det} Q)^{2}}+\frac{N^{2 k-1} \sqrt{\tilde{a}}}{\operatorname{det} Q}+N^{k-1} .
$$

The negative power of $t_{1}=\min T$ saves us only one power in $N$. The estimate above is sufficient in order to prove Theorem 6 as it is smaller than the bound for the theta series in the $R(2)$ case. Nonetheless, we take a closer look to obtain a slightly better bound. This might prove useful in the future, when there is a better treatment available for the theta series.

Lemma 57. Consider $A_{3}(T), B_{3}(T)$ as above. Then

$$
N^{4 k-3} \sum_{\substack{t_{1}, t_{2} t_{4} \ll N^{2+\epsilon} \\\left|t_{2}\right| \leqslant t_{1}}} \frac{A_{1}(T)^{2}+A_{2}(T)^{2}}{(\operatorname{det} T)^{2 k-3}} \ll \frac{N^{2 k+2}}{(\operatorname{det} Q)^{2}}+\frac{N^{2 k-1}}{\operatorname{det} Q}+\frac{N^{2}}{a} .
$$


Proof. We proceed similarly as in the proof of Theorem 56. For the Fourier coefficients $a(n)$ of $\theta\left(N Q^{-1}, z\right)-\theta$ (gen $\left.N Q^{-1}, z\right)$ we have two options, we can apply Lemma 24 and the Petersson formula which gives

$$
a(n) \ll\left(\frac{\sqrt{\operatorname{det} Q}}{N^{\frac{k}{2}}}+\sqrt[4]{\tilde{a}}\right) n^{\frac{k-1}{2}}\left(1+\frac{n^{\frac{1}{4}}(n, N)^{\frac{1}{4}}}{N^{\frac{1}{2}}}\right)
$$

or (65) and (61) which yields

$$
a(n) \ll 1+\frac{n^{\frac{1}{2}} \sqrt{a_{2 k-2}}}{N} \ldots+\frac{n^{k-1} \sqrt{\operatorname{det} Q}}{N^{k-1} \sqrt{a_{2 k} a_{2 k-1}}}+\frac{n^{k-1} \sqrt{\operatorname{det} Q} \sqrt{(n, N)}}{N^{k}} .
$$

Recall that we have to estimate

$$
\sum_{\left(w_{1}, w_{3}\right)=1}\left(T[w]_{1}\right)^{1-k} a\left(\frac{T[w]_{1}}{v_{1}^{2}}\right)
$$

where $T[w]_{1}=w_{1}^{2} t_{1}+w_{1} w_{3} t_{2}+w_{3}^{2} t_{4}$. We assume that $T \in \mathcal{R}_{2}$ so that $t_{1}=\min T$. If we apply the second estimate for all $w_{1}, w_{3}$ these sums do not converge anymore. Hence, we apply it only for $w_{3}=0$ (which implies $t_{1}=\min T$ ) and the first estimate in all other cases:

$$
\begin{aligned}
A_{3}(T) & \leqslant N^{-k}(\operatorname{det} Q)^{-\frac{1}{2}} \sum_{\left(w_{1}, w_{3}\right)=1}\left(T[w]_{1}\right)^{1-k}\left(r\left(N Q^{-1}, \frac{T[w]_{1}}{v_{1}^{2}}\right)-r\left(\operatorname{gen} N Q^{-1}, \frac{T[w]_{1}}{v_{1}^{2}}\right)\right) \\
& \ll(\operatorname{det} T)^{k-\frac{3}{2}} N^{-k}\left(\frac{(\min T)^{1-k}}{\sqrt{\operatorname{det} Q}}+\ldots+\frac{1}{N^{k-1} \sqrt{a_{2 k} a_{2 k-1}}}+\frac{(\min T, N)}{N^{k}}\right) \\
& +(\operatorname{det} T)^{\frac{3}{4} k-\frac{5}{4}} N^{-k}\left(\frac{\sqrt[4]{a_{1} a_{2}}}{\sqrt{\operatorname{det} Q}}+N^{-\frac{k}{2}}\right)\left(1+\frac{(\operatorname{det} T)^{\frac{1}{8}}}{N^{\frac{1}{4}}}\right), \\
B_{3}(T) & \ll(\operatorname{det} T)^{k-\frac{3}{2}} N^{-k} \frac{N^{\frac{3}{2}}(N, T)^{\frac{1}{2}}}{\sqrt{\operatorname{det} Q}}\left(\frac{(\min T)^{1-k}}{\sqrt{\operatorname{det} Q}}+\ldots+\frac{1}{N^{k-1} \sqrt{a_{2 k} a_{2 k-1}}}+\frac{(\min T, N)}{N^{k}}\right) \\
& +(\operatorname{det} T)^{k-\frac{3}{2}}\left(\frac{N^{2-\frac{3}{2} k}}{\operatorname{det} Q}+\frac{N^{2-2 k}}{\sqrt{\operatorname{det} Q}}\right) \\
& +(\operatorname{det} T)^{\frac{3}{4} k-\frac{5}{4}} N^{-k}\left(\frac{N^{2-k+\epsilon}}{\sqrt{\operatorname{det} Q}}+\frac{N^{\frac{3}{2}+\epsilon}}{\operatorname{det} Q \sqrt[4]{a_{2 k} a_{2 k-1}}}\right)\left(1+\frac{(\operatorname{det} T)^{\frac{1}{8}}}{N^{\frac{1}{4}}}\right) .
\end{aligned}
$$

The case $w_{3} \neq 0$ gives the term with respect to $(\operatorname{det} T)^{\frac{3}{4} k-\frac{5}{4}}$. In the case of $N \mid v_{1}$ we save a considerable power in $N$ by $N \mid t_{1}$ as illustrated by the second line in the bound of $A_{2}(T)$. The claim follows now by inserting the bounds in the formula above.

Finally, we address the representatives in $R(1)$ and $R(2)$. To this end, we use the decomposition from Lemma 48. This gives the following bounds: 
Lemma 58. Let

$$
E\left(f_{1}, Z\right) \mid\left[\left[\iota_{1}(J)\right]=\sum_{T \in \mathcal{S}} A_{1}(T) e\left(\frac{\operatorname{tr}(T Z)}{N}\right) .\right.
$$

Then, for $T>0$ it holds that

$$
A_{1}(T) \ll(\operatorname{det} T)^{k-\frac{3}{2}} N^{2-2 k}\left(\frac{N^{\frac{k}{2}}}{\sqrt{\operatorname{det} Q}}+\frac{N^{\frac{1}{2}}}{\sqrt[4]{a}}\right) .
$$

Proof. We use that

$$
\begin{aligned}
& A_{1}(T) \exp \left(-\frac{2 \pi \operatorname{tr}(T Y)}{N}\right)=N^{-3} \int_{X} \sum_{M \in P(N) \backslash \Gamma_{0}(N) \iota_{1}(J)}\left(f_{1} \| M\right)(Z) e\left(-\frac{\operatorname{tr}(T X)}{N}\right) d X \\
= & N^{-3} \int_{X} \sum_{\sigma, v, w, \mu, L}\left(f_{1} \mid[\omega(L)] \|[\gamma(v, \sigma)]\right)\left(X+\mu+i w^{-1} Y w^{-T}\right) e\left(-\frac{\operatorname{tr}\left(w^{T} T w X\right)}{N}\right) d X
\end{aligned}
$$

where $X \in \mathcal{P}(\mathbb{Z} / N \mathbb{Z}), \mu_{2}, \mu_{4} \in N \mathbb{Z}, \sigma, v, w, L$ as in Lemma 48 and 44 (with $\left.N_{1}=N_{2}=N\right)$. We denote the entries of $w^{-1} Y w^{-T}$ and $T[w]$ by $\tilde{y}_{1}, \tilde{y}_{2}, \tilde{y}_{4}$ and $T[w]_{1}, T[w]_{2}, T[w]_{4}$.

We treat the different cases of Lemma 48 separately. We start with case $(d)$ since this is the only case with $N \mid c_{1}$. This implies that $L=I_{4}$ and we put $f_{1}(z)=a(n) e(n z)$. The further conditions of this case are $N \mid w_{1}$ and $N \mid d$. We compute the contribution analogously to Lemma 50 by

$$
\begin{aligned}
& N^{-3} \sum_{c \geqslant 1} \sum_{v_{1} \geqslant 1} \sum_{\substack{\left(w_{1}, w_{3}\right)=1 \\
w_{1} \geqslant 0, N \mid w_{1}}} c^{-k} \sum_{n} a(n) e\left(n\left(i \tilde{y}_{1} v_{1}^{2}+a c^{-1} v_{2}^{2}\right)\right) \int_{0}^{N} e\left(\left(n N v_{1}^{2}-T[w]_{1}\right) \frac{x_{1}}{N}\right) d x_{1} \\
& \times \sum_{\substack{d \bmod N c \\
N \mid d, a d \equiv 1 \bmod c}} \sum_{\substack{v_{2} \bmod N c v_{1} \\
\left(v_{1}, v_{2}\right)=1}} \int_{x_{2}, x_{4} \in \mathbb{R}}\left(x_{4}+\tilde{y}_{4}+c^{-1} d\right)^{-k} e\left(-\frac{T[w]_{2} x_{2}}{N}-\frac{T[w]_{4} x_{4}}{N}\right) \\
& \times e\left(-n\left(\left(v_{1}\left(x_{2}+i \tilde{y}_{2}\right)-c^{-1} v_{2}\right)^{2}\left(x_{4}+i \tilde{y}_{4}+c^{-1} d\right)^{-1}\right)\right) d x_{2} d x_{4} .
\end{aligned}
$$

We substitute $x_{2}$ by $N x_{2}$ and $x_{4}$ by $N x_{4}$. Proceeding as in Lemma 50 we obtain

$$
\begin{aligned}
& =(\operatorname{det} T)^{k-\frac{3}{2}} N^{-k} \exp \left(-\frac{2 \pi \operatorname{tr}(T Y)}{N}\right) C_{k} \sum_{c \geqslant 1} c^{-k} \sum_{\substack{\left(w_{1}, w_{3}\right)=1 \\
w_{1} \geqslant 0, N\left|w_{1} N v_{1}^{2}\right| T[w]_{1}}} \sum_{\substack{v_{1} \geqslant 1 \\
N v_{1}^{2}}} a\left(\frac{T[w]_{1}}{N c}\right) \\
& \times\left(T[w]_{1}\right)^{1-k} \sum_{\substack{d \bmod N c \\
N \mid d, a d \equiv 1 \bmod c}} \sum_{\substack{v_{2} \bmod N c v_{1} \\
\left(v_{1}, v_{2}\right)=1}} e\left(\frac{T[w]_{1} v_{1}^{-2} a v_{2}^{2}-T[w]_{2} v_{2} v_{1}^{-1}+T[w]_{4} d}{N c}\right)
\end{aligned}
$$

where $C_{k}$ is given by (95). Note that $N \mid T\left[w_{1}\right]=w_{1}^{2} t_{1}+w_{1} w_{3} t_{2}+w_{3}^{2} t_{4}$ implies that $N \mid t_{4}$. 
We bound $a\left(\frac{T[w]_{1}}{N v_{1}^{2}}\right)$ by the Petersson formula and $\left\langle f_{1}, f_{1}\right\rangle \ll \frac{N^{k}}{\operatorname{det} Q}+\frac{N}{\sqrt{a}}$. Furthermore, we estimate the $d$ sum by $c$, the $v_{1}$ sum by $N c v_{1}$ and $\left(T[w]_{1}\right)^{1-k} \leqslant N^{1-k}$. This gives a contribution of at most

$$
(\operatorname{det} T)^{k-\frac{3}{2}} N^{2-2 k}\left(\frac{N^{\frac{k}{2}}}{\sqrt{\operatorname{det} Q}}+\frac{N^{\frac{1}{2}}}{\sqrt[4]{a}}\right) .
$$

This forms the main error term. In all other cases we put

$$
f \mid[\omega(L)](z)=\sum_{n} b(n) e\left(\frac{n d_{1}}{N}\right) e\left(\frac{n z}{N}\right)
$$

where $b(n)$ denote the Fourier coefficients of $N^{-\frac{k}{2}} f \mid[\eta](z)=\sum_{n} b(n) e(n z)$ and $d_{1}$ is the lower right entry of $\omega(L)$. We compute the integral in the same way as above. The main difference is that the $x_{1}$ integral equals

$$
\int_{0}^{N} e\left(\left(n v_{1}^{2}-T[w]_{1}\right) \frac{x_{1}}{N}\right) d x_{1}
$$

which implies that $n=\frac{T[w]_{1}}{v_{1}^{2}}$ and thus, $v_{1}^{2} \mid T[w]_{1}$. Apart from that, everything works completely analogously and as before, we obtain a factor of size $N^{-k}$ from the term $\left(N x_{4}+\tilde{y}_{4}+c^{-1} d\right)^{-k}$. Consequently, the contribution is given by

$$
\begin{aligned}
& (\operatorname{det} T)^{k-\frac{3}{2}} N^{-k} \exp \left(-\frac{2 \pi \operatorname{tr}(T Y)}{N}\right) C_{k} \sum_{c \geqslant 1} c^{-k} \sum_{\substack{\left(w_{1}, w_{3}\right)=1 \\
w_{1} \geqslant 0}} \sum_{\substack{v_{1} \geqslant 1 \\
v_{1}^{2} \mid T[w]_{1}}} b\left(\frac{T[w]_{1}}{v_{1}^{2}}\right)\left(T[w]_{1}\right)^{1-k} \\
& \times \sum_{\substack{d \bmod N c{ }_{a} c \\
a d \equiv 1 \bmod c}} \sum_{\substack{v_{2} \bmod N c v_{1} \\
\left(v_{1}, v_{2}\right)=1}} e\left(\frac{T[w]_{1} v_{1}^{-2} a v_{2}^{2}-T[w]_{2} v_{2} v_{1}^{-1}+T[w]_{4} d}{N c}\right) e\left(\frac{d_{1} T[w]_{1} v_{1}^{-2}}{N}\right)
\end{aligned}
$$

with the respective congruence conditions of each case $(a),(b),(c)$. To estimate the Fourier coefficients $b(n)$ we apply the Petersson formula and

$$
N^{-k}\langle f|[\eta], f|[\eta]\rangle \ll\left(N^{-k}+\frac{\sqrt{\tilde{a}}}{\operatorname{det} Q}\right) N^{\epsilon} .
$$

For case $(a)$, we note that $N \mid v_{1}$ implies that $N^{2} \mid T[w]_{1}$. Hence, its contribution is bounded by

$$
(\operatorname{det} T)^{k-\frac{3}{2}} N^{5-3 k}\left(\frac{\sqrt[4]{\tilde{a}}}{\sqrt{\operatorname{det} Q}}+N^{-\frac{k}{2}}\right) N^{\epsilon}
$$

By $N \mid c$, case $(b)$ and $(c)$ contribute at most

$$
(\operatorname{det} T)^{k-\frac{3}{2}} N^{4-2 k}\left(\frac{\sqrt[4]{\tilde{a}}}{\sqrt{\operatorname{det} Q}}+N^{-\frac{k}{2}}\right) N^{\epsilon} .
$$


Corollary 59. Let

$$
E_{N}\left(f_{2}, Z\right) \mid\left[\iota_{2}(J)\right]=\sum_{T \in \mathcal{S}} B_{2}(T) e\left(\frac{\operatorname{tr} T Z}{N}\right) .
$$

Then, for $T>0$, it holds that

$$
B_{2}(T) \ll(\operatorname{det} T)^{k-\frac{3}{2}} N^{2-2 k}\left(\frac{N^{\frac{k}{2}}}{\sqrt{\operatorname{det} Q}}+\frac{N^{k} \sqrt[4]{\tilde{a}}}{\operatorname{det} Q}\right) .
$$

Proof. We apply the following trick:

$$
E_{N}\left(f_{2}, Z\right)\left|\left[\iota_{2}(J)\right]=E\left(f_{2},\left(\begin{array}{cc}
N z_{1} & z_{2} \\
z_{2} & N^{-1} z_{4}
\end{array}\right)\right)\right|\left[\iota_{1}(J)\right] .
$$

If $E_{N}\left(f_{2}, Z\right) \mid\left[\iota_{2}(J)\right]=\sum_{T \in \mathcal{S}} B_{2}(T) e\left(\frac{\operatorname{tr}(T Z)}{N}\right)$ with $t_{1}, t_{2} \equiv 0(\bmod N)$, then,

$$
E\left(f_{2}, Z\right) \mid\left[\iota_{1}(J)\right]=\sum_{0 \leqslant T \in \mathrm{P}_{2}} B_{2}\left(\begin{array}{cc}
N^{-1} t_{1} & t_{2} / 2 \\
t_{2} / 2 & N t_{4}
\end{array}\right) e\left(\frac{\operatorname{tr}(T Z)}{N}\right) .
$$

Let $T>0$. Then,

$$
B_{2}(T) e\left(-\frac{2 \pi \operatorname{tr}(T Z)}{N}\right)=N^{-3} \int_{X} \sum_{\substack{M \in P(N) \backslash \Gamma_{0}(N) \iota_{1}(J) \\ \lambda(M) \neq(0,0, *, *)}}\left(f_{2} \| M\right)(Z) e\left(-\frac{2 \pi \operatorname{tr}(\tilde{T} X)}{N}\right) d X
$$

with $\tilde{T}=\left(\begin{array}{cc}N^{-1} t_{1} & t_{2} / 2 \\ t_{2} / 2 & N t_{4}\end{array}\right) \in \mathscr{S}$ and $X \in \mathcal{P}(\mathbb{Z} / N \mathbb{Z})$. This expression can be estimated exactly as in the previous lemma. The only difference is that we need to consider $f_{2}$ and $\tilde{T}$ instead of $f_{1}$ and $T$. The latter does not impact our estimates as $\operatorname{det} \tilde{T}=\operatorname{det} T$ and we apply the same trivial bound for $\tilde{T}[w]_{1}^{1-k}$ as we $\operatorname{did}$ for $\left(T[w]_{1}\right)^{1-k}$, that is $\tilde{T}[w]_{1} \geqslant\left(N^{2}, \tilde{T}[w]_{1}\right)$.

Recall that $f_{2}(z)=\frac{N^{k}}{\operatorname{det} Q}\left(\theta\left(N Q^{-1}, z\right)-\theta\left(\right.\right.$ gen $\left.\left.N Q^{-1}, z\right)\right)$. For case $(d)$ we use

$$
\left\langle f_{2}, f_{2}\right\rangle \ll \frac{N^{k}}{\operatorname{det} Q}+\frac{N^{2 k} \sqrt{\tilde{a}}}{(\operatorname{det} Q)^{2}} .
$$

This gives a contribution of at most

$$
(\operatorname{det} T)^{k-\frac{3}{2}} N^{2-2 k}\left(\frac{N^{\frac{k}{2}}}{\sqrt{\operatorname{det} Q}}+\frac{N^{k} \sqrt[4]{\tilde{a}}}{\operatorname{det} Q}\right)
$$

In all other cases we use

$$
N^{-k}\left\langle f_{2}\left|[\eta], f_{2}\right|[\eta]\right\rangle \ll \frac{N^{k}}{(\operatorname{det} Q)^{2}}+\frac{N}{\operatorname{det} Q \sqrt{a}}
$$


Consequently, the contribution of $(a)$ is bounded by

$$
(\operatorname{det} T)^{k-\frac{3}{2}} N^{5-3 k}\left(\frac{N^{\frac{k}{2}}}{\operatorname{det} Q}+\frac{N^{\frac{1}{2}}}{\sqrt{\operatorname{det} Q} \sqrt[4]{a}}\right)
$$

and $(b)$ and $(c)$ contribute at most

$$
(\operatorname{det} T)^{k-\frac{3}{2}} N^{4-2 k}\left(\frac{N^{\frac{k}{2}}}{\operatorname{det} Q}+\frac{N^{\frac{1}{2}}}{\sqrt{\operatorname{det} Q \sqrt[4]{a}}}\right) .
$$

The remaining two Klingen-Eisenstein series can be treated very similarly:

Lemma 60. Let

$$
\begin{aligned}
& E\left(f_{1}, Z\right) \mid\left[\left[\iota_{2}(J)\right]=\sum_{T \in \mathcal{S}} A_{2}(T) e\left(\frac{\operatorname{tr}(T Z)}{N}\right)\right. \\
& \left.E_{N}\left(f_{2}, Z\right) \mid \iota_{1}(J)\right]=\sum_{T \in \mathcal{S}} B_{1}(T) e\left(\frac{\operatorname{tr}(T Z)}{N}\right) .
\end{aligned}
$$

Then, we have for $T>0$ that

$$
\begin{aligned}
& A_{2}(T) \ll(\operatorname{det} T)^{k-\frac{3}{2}} N^{2-2 k}\left(\frac{N^{\frac{k}{2}}}{\sqrt{\operatorname{det} Q}}+\frac{N^{\frac{1}{2}}}{\sqrt[4]{a}}\right), \\
& B_{1}(T) \ll(\operatorname{det} T)^{k-\frac{3}{2}} N^{2-2 k}\left(\frac{N^{\frac{k}{2}}}{\sqrt{\operatorname{det} Q}}+\frac{N^{k} \sqrt[4]{\tilde{a}}}{\operatorname{det} Q}\right) .
\end{aligned}
$$

Proof. Similarly to before, we use that

$$
\begin{aligned}
& A_{2}(T) \exp \left(-\frac{2 \pi \operatorname{tr}(T Y)}{N}\right)=N^{-3} \int_{X} \sum_{M \in P(N) \backslash \Gamma_{0}(N) \iota_{2}(J)}\left(f_{1} \| M\right)(Z) e\left(-\frac{\operatorname{tr}(T X)}{N}\right) d X \\
= & N^{-3} \int_{X} \sum_{\sigma, v, w, \mu, L}(f \mid[\omega(L)] \|[\gamma(v, \sigma)])\left(X+\mu+i w^{-1} Y w^{-T}\right) e\left(-\operatorname{tr} w^{T} T w X\right) d X
\end{aligned}
$$

where $X \in \mathcal{P}(Z / N \mathbb{Z}), \mu_{2}, \mu_{4} \in N \mathbb{Z}, \sigma, v, w, L$ as in Lemma 48 and 44 (with $N_{1}=$ $\left.N_{2}=N\right)$.

As above, we treat the different cases of Lemma 48 separately. We start with case $(h)$ since this is the only case with $N \mid c_{1}$. This implies that $L=I_{4}$ and we put $f_{1}(z)=a(n) e(n z)$. The further conditions of this case are $N \mid w_{3}$ and $N \mid d$. 
We compute the contribution as in Lemma 58 which gives

$$
\begin{aligned}
& (\operatorname{det} T)^{k-\frac{3}{2}} N^{-k} \exp \left(-\frac{2 \pi \operatorname{tr}(T Y)}{N}\right) C_{k} \sum_{c \geqslant 1} c^{-k} \sum_{\substack{\left(w_{1}, w_{3}\right)=1 \\
w_{1} \geqslant 0, N \mid w_{3}}} \sum_{\substack{v_{1} \geqslant 1 \\
N v_{1}^{2} \mid T[w]_{1}}} a\left(\frac{T[w]_{1}}{N v_{1}^{2}}\right) \\
& \times\left(T[w]_{1}\right)^{1-k} \sum_{\substack{d \bmod N c \\
N \mid d, a d \equiv 1 \bmod c}} \sum_{\substack{v_{2} \bmod N c v_{1} \\
\left(v_{1}, v_{2}\right)=1}} e\left(\frac{T[w]_{1} v_{1}^{-2} a v_{2}^{2}-T[w]_{2} v_{2} v_{1}^{-1}+T[w]_{4} d}{N c}\right) .
\end{aligned}
$$

Note that $N \mid T\left[w_{1}\right]=w_{1}^{2} t_{1}+w_{1} w_{3} t_{2}+w_{3}^{2} t_{4}$ directly implies that $N \mid t_{1}$. Again, we estimate $\left(T[w]_{1}\right)^{1-k} \leqslant N^{1-k}$.

We proceed as in Lemma 58 and obtain a contribution of at most

$$
(\operatorname{det} T)^{k-\frac{3}{2}} N^{2-2 k}\left(\frac{N^{\frac{k}{2}}}{\sqrt{\operatorname{det} Q}}+\frac{N^{\frac{1}{2}}}{\sqrt[4]{a}}\right)
$$

This is the main error term. The other cases work exactly as before and we get for $(f),(g)$ and $(h)$ the same error terms as in Lemma 58 for the cases $(a),(b)$ and (c) respectively.

For $B_{1}(T)$ we apply that

$$
E_{N}\left(f_{2}, Z\right)\left|\left[\iota_{1}(J)\right]=E\left(f_{2},\left(\begin{array}{cc}
N^{-1} z_{1} & z_{2} \\
z_{2} & N z_{4}
\end{array}\right)\right)\right|\left[\iota_{2}(J)\right]
$$

Hence,

$$
B_{1}(T) \exp \left(-\frac{2 \pi i \operatorname{tr}(T Y)}{N}\right)=N^{-3} \int_{X} \sum_{\substack{M \in P(N) \backslash \Gamma_{0}(N) \iota_{2}(J) \\ \lambda(M) \neq(0,0, *, *)}}\left(f_{2} \| M\right)(Z) e\left(-\frac{\operatorname{tr}\left(T^{\prime} X\right)}{N}\right) d X
$$

with $T^{\prime}=\left(\begin{array}{cc}N t_{1} & t_{2} / 2 \\ t_{2} / 2 & \frac{t_{4}}{N}\end{array}\right)$ and $X \in \mathcal{P}(\mathbb{R} / N \mathbb{Z})$. The rest works exactly as above, bearing in mind that $f_{2}(z)=\frac{N^{k}}{\operatorname{det} Q}\left(\theta\left(N Q^{-1}, z\right)-\theta\left(\operatorname{gen} N Q^{-1}, z\right)\right)$.

By Lemma 39 the Klingen-Eisenstein series contribution to $\langle G, G\rangle$ for the $R(1)$ class is bounded by

$$
N^{2 k-2} \sum_{\substack{T \in \mathscr{S}, t_{1}, t_{2}, t_{4} \ll N^{1+\epsilon} \\ t_{2}, t_{4}=0(\bmod N)}} \frac{\left|A_{1}(T)+B_{1}(T)\right|^{2}}{(\operatorname{det} T)^{k-\frac{3}{2}}} \ll\left(\frac{N^{2 k} \sqrt{\tilde{a}}}{(\operatorname{det} Q)^{2}}+\frac{N}{\sqrt{a}}\right) N^{\epsilon},
$$


and for the $R(2)$ class with $U=\left(\begin{array}{ll}1 & u \\ & 1\end{array}\right)$ by

$$
N^{2 k-2} \sum_{u=0}^{n-1} \sum_{\substack{T \in \mathscr{S}, t_{1}, t_{2}, t_{4} \ll N^{1+\epsilon} \\ t_{1}, t_{2}=0(\bmod N)}} \frac{\left|A_{2}\left(U T U^{T}\right)+B_{2}\left(U T U^{T}\right)\right|^{2}}{(\operatorname{det} T)^{k-\frac{3}{2}}} \ll\left(\frac{N^{2 k+1} \sqrt{\tilde{a}}}{(\operatorname{det} Q)^{2}}+\frac{N^{2}}{\sqrt{a}}\right) N^{\epsilon}
$$

\subsubsection{Bounds for Fourier coefficients of theta series}

The aim of this section is to estimate the contribution of theta and genus theta series to the inner product of $G(Z)=\theta(Q, Z)-\theta($ gen $Q, Z)-E\left(f_{1}, Z\right)-E_{N}\left(f_{2}, Z\right)$. As done in the case of Klingen-Eisenstein series we treat the coset classes $R(0)$, $R(1), R(2)$ and $R(3)$ from Lemma 36 one by one. To this end, recall that

$$
r(Q, T)=\#\left\{x, y \in \mathbb{Z}^{2 k} \mid T=\left(\begin{array}{ll}
x^{T} Q x & x^{T} Q y \\
y^{T} Q x & y^{T} Q y
\end{array}\right)\right\}
$$

For given $t_{1}, t_{4}$ we count solutions $x_{1}, x_{2}, y_{1}, y_{2} \in \mathbb{Z}^{2 k}$ of

$$
t_{1}=x_{1}^{T} Q x_{1}=x_{1}^{T} Q x_{1}, t_{4}=y_{1}^{t} Q y_{1}=y_{2}^{t} Q y_{2}
$$

with the additional requirement that $t_{1}=x_{1}^{T} Q y_{1}=x_{2}^{T} Q y_{2}$. This already determines $t_{2}$ and by dropping $x_{1}^{T} Q y_{1}=x_{2}^{T} Q y_{2}$ we obtain for $l \in \mathbb{R}$ that

$$
\sum_{t_{1}, t_{2}, t_{4} \leqslant l} r(Q, T)^{2} \leqslant \sum_{t_{1}, t_{4} \leqslant l} r\left(Q, t_{1}\right)^{2} r\left(Q, t_{4}\right)^{2}
$$

By definition, the very same result holds for $r($ gen $Q, T)$. It follows by Lemma 22 , 37 , and (61) that the contribution of theta and genus theta series in the $R(0)$ case is bounded by a constant.

Next, we address the $R(3)$ class. By the transformation formula it holds that

$$
\theta(Q, Z) \mid\left[J_{4}\right]=(\operatorname{det} Q)^{-1} \theta\left(Q^{-1}, Z\right) .
$$

Thus, the Fourier expansion is given by

$$
\theta(Q, Z) \mid\left[J_{4}\right]=\sum_{T \in \mathcal{S}} \frac{r\left(N Q^{-1}, T\right)}{\operatorname{det} Q} e\left(\frac{\operatorname{tr}(T Z)}{N}\right) .
$$

According to Lemma 38 we need to estimate

$$
\sum_{t_{1}, t_{2}, t_{4} \ll N^{1+\epsilon}} \frac{r\left(N Q^{-1}, T\right)^{2}+r\left(\operatorname{gen} N Q^{-1}, T\right)^{2}}{(\operatorname{det} Q)^{2}} .
$$


As $r(Q, T)=r\left(Q, U^{T} T U\right)$ for $U \in \mathrm{GL}_{2}(\mathbb{Z})$, we may assume that $T \in \mathcal{R}_{2}$. This simplifies the evaluation of $r\left(N Q^{-1}, T\right)^{2}$ since $t_{1} t_{4}=\operatorname{det} T$, but comes at the cost that $t_{4} \ll \frac{\operatorname{det} T}{t_{1}}$ which implies for small $t_{1}=\min T$ that $t_{4}$ might get as large as $N^{2+\epsilon}$. The following result balances these two effects:

Lemma 61. Let $Q$ be positive definite $2 k \times 2 k$ matrix and $0<T \in \mathcal{S}(\mathbb{Z})$ and $\alpha \in[1,2]$. Then,

$\sum_{t_{1}, t_{2}, t_{4} \leqslant N} \frac{\left(r\left(N Q^{-1}, T\right)+r\left(\operatorname{gen} N Q^{-1}, T\right)\right)^{2}}{(\operatorname{det} T)^{k-\frac{3}{2}}(\operatorname{det} Q)^{2}} \ll \frac{1}{(\operatorname{det} Q)^{2}}+\frac{N^{(\alpha-2) k+1+\frac{\alpha}{2}}}{\operatorname{det} Q^{1+\frac{1}{2 k}}}+\frac{N^{-\alpha\left(k-\frac{3}{2}\right)}}{(\operatorname{det} Q)^{\frac{1}{k}}}$.

Proof. We make a case distinction according to the size of $\operatorname{det} T$. If $\operatorname{det} T \leqslant N^{\alpha}$ we assume that $T \in \mathcal{R}_{2}$ and estimate by 100 :

$$
\sum_{T \in \mathcal{R}_{2}} \frac{r\left(N Q^{-1}, T\right)^{2}}{\operatorname{det} T} \ll \sum_{\substack{t_{1} t_{4} \leqslant N^{\alpha} \\ t_{1} \leqslant t_{4}}} \frac{r\left(N Q^{-1}, t_{1}\right)^{2}}{t_{1}^{k-\frac{3}{2}}} \frac{r\left(N Q^{-1}, t_{4}\right)^{2}}{t_{4}^{k-\frac{3}{2}}} .
$$

The largest term comes from the case when $t_{1}=\min T$ is small and $t_{4}$ is large. By Lemma 22 with $m=2 k$, we obtain

$$
\begin{aligned}
\sum_{t_{4} \leqslant N^{\alpha}} \frac{r\left(N Q^{-1}, t_{4}\right)^{2}}{t_{4}^{k-\frac{3}{2}}} & \ll\left(1+\ldots+\frac{N^{\alpha\left(k+\frac{1}{2}\right)} \operatorname{det} Q}{N^{2 k-1} \sqrt{a_{m} a_{m-1}}}\right) N^{\epsilon} \\
& \ll\left(1+N^{(\alpha-2) k+1+\frac{\alpha}{2}}(\operatorname{det} Q)^{1-\frac{1}{2 k}}\right) N^{\epsilon} .
\end{aligned}
$$

For the remaining matrices, we use 100$), \operatorname{det} T \geqslant N^{\alpha}$ and Lemma 22 which gives a bound of size

$N^{-\alpha\left(k-\frac{3}{2}\right)} \sum_{t_{1} \ll N^{1+\epsilon}} r\left(N Q^{-1}, t_{1}\right)^{2} \sum_{t_{4} \ll N^{1+\epsilon}} r\left(N Q^{-1}, t_{4}\right)^{2} \ll N^{-\alpha\left(k-\frac{3}{2}\right)}\left(1+(\operatorname{det} Q)^{2-\frac{1}{k}}\right) N^{\epsilon}$.

For the genus theta series, we apply 61

$$
\begin{aligned}
\sum_{t_{1}, t_{2}, t_{4} \leqslant N^{1+\epsilon}} \frac{r\left(\operatorname{gen} N Q^{-1}, T\right)^{2}}{(\operatorname{det} T)^{k-\frac{3}{2}}} & \ll \sum_{t_{1} \leqslant N^{1+\epsilon}} r\left(\operatorname{gen} N Q^{-1}, t_{1}\right)^{2} \sum_{t_{4} \leqslant N^{1+\epsilon}} r\left(\operatorname{gen} N Q^{-1}, t_{4}\right)^{2} \\
& \ll \frac{(\operatorname{det} Q)^{2}}{N^{2 k-1}} N^{\epsilon} .
\end{aligned}
$$

By Lemma 38, the theta series contributes in the $R(3)$ case at most

$$
\begin{array}{r}
N^{2 k} \sum_{t_{1}, t_{2}, t_{4} \ll N^{1+\epsilon}} \frac{r\left(N Q^{-1}, T\right)^{2}+r\left(\operatorname{gen} N Q^{-1}, T\right)^{2}}{(\operatorname{det} Q)^{2}(\operatorname{det} T)^{k-\frac{3}{2}}} \\
\ll \frac{N^{2 k}}{(\operatorname{det} Q)^{2}}+\frac{N^{\alpha\left(k+\frac{1}{2}\right)+1}}{\operatorname{det} Q^{1+\frac{1}{4 k}}}+\frac{N^{(2-\alpha) k+\frac{3 \alpha}{2}}}{\operatorname{det} Q^{\frac{1}{k}}} .
\end{array}
$$


We match the second and third error term roughly by setting

$$
\alpha=\frac{4 k-(i+2)+\frac{i}{2 k}}{2 k-1}
$$

where $i$ is given by $N^{2 k-i}=\operatorname{det} Q$. Thus, these two terms are bounded by $N^{\frac{3+i}{2}}$. In summary, the contribution in the $R(3)$ case of theta series to $\langle G, G\rangle$ is bounded by

$$
\frac{N^{2 k}}{(\operatorname{det} Q)^{2}}+\frac{N^{k+\frac{3}{2}}}{\sqrt{\operatorname{det} Q}}
$$

As before, we treat the representatives of $R(1)$ and $R(2)$ simultaneously. For this purpose, we introduce the following transformation formula:

Lemma 62. We have

$$
\theta(Q, Z) \mid\left[\iota_{1}(J)\right]=\sum_{x_{1}, x_{2} \in \mathbb{Z}^{2 k}} \alpha\left(x_{1}, x_{2}, Q\right) e\left(\frac{1}{2} x_{1}^{T} Q^{-1} x_{1} z_{1}+x_{1} x_{2} z_{2}+\frac{1}{2} x_{2}^{T} Q x_{2} z_{4}\right)
$$

with $\left|\alpha\left(x_{1}, x_{2}, Q\right)\right|=(\operatorname{det} Q)^{-1 / 2}$ and

$$
\theta(Q, Z) \mid\left[\iota_{2}(J)\right]=\sum_{x_{1}, x_{2} \in \mathbb{Z}^{2 k}} \beta\left(x_{1}, x_{2}, Q\right) e\left(\frac{1}{2} x_{1}^{T} Q x_{1} z_{1}+x_{1} x_{2} z_{2}+\frac{1}{2} x_{2}^{T} Q^{-1} x_{2} z_{4}\right)
$$

with $\left|\beta\left(x_{1}, x_{2}, Q\right)\right|=(\operatorname{det} Q)^{-1 / 2}$.

Proof. We write $\iota_{1}(J)$ and $\iota_{2}(J)$ as

$$
-\left(\begin{array}{cc}
I & M \\
& I
\end{array}\right) J_{4}\left(\begin{array}{cc}
I & S \\
& I
\end{array}\right) J_{4}\left(\begin{array}{cc}
I & K \\
& I
\end{array}\right)
$$

where we put

$$
M, S, K=\left(\begin{array}{cc}
-1 & 0 \\
0 & 0
\end{array}\right) \quad \text { and } \quad M, S, K=\left(\begin{array}{cc}
0 & 0 \\
0 & -1
\end{array}\right)
$$

respectively. To hold notation simple, we set $\tau_{S}=\left(\begin{array}{cc}I & S \\ & I\end{array}\right)$. By the transformation formula of the theta series, we get

$$
\begin{aligned}
\theta(Q, Z) \mid\left[\tau_{M} J \tau_{S}\right] & =(\operatorname{det} Q)^{-1} \theta\left(Z+S, Q^{-1}\right) \\
& =(\operatorname{det} Q)^{-1} \sum_{X \in M_{2 k, 2}(\mathbb{Z})} e\left(\frac{1}{2} \operatorname{tr}\left(X^{T} Q^{-1} X(Z+S)\right)\right) .
\end{aligned}
$$


We put $X=V+Q U$ with $V \in M_{2 k, 2}(\mathbb{Z}) / Q M_{2 k, 2}(\mathbb{Z})$ and $U \in M_{2 k, 2}(\mathbb{Z})$. Thus, the previous display equals

$$
(\operatorname{det} Q)^{-1} \sum_{V \in M_{2 k, 2}(\mathbb{Z}) / Q M_{2 k, 2}(\mathbb{Z})} e\left(\frac{1}{2} \operatorname{tr}\left(V^{T} Q^{-1} V S\right)\right) \theta_{\left(Q^{-1} V, 0\right)}(Q, Z),
$$

where $\theta_{(A, B)}(Q, Z)$ is the generalized theta series from Section 4.1.2. By its transformation formula, (78), we conclude

$$
\begin{aligned}
& \theta(Q, Z) \mid\left[\tau_{M} J \tau_{S} J\right]=(\operatorname{det} Q)^{-2} \sum_{V} e\left(\frac{1}{2} \operatorname{tr}\left(V^{T} Q^{-1} V S\right)\right) \theta_{\left(0, Q^{-1} V\right)}\left(Q^{-1}, Z\right) \\
& =(\operatorname{det} Q)^{-2} \sum_{X \in M_{2 k, 2}(\mathbb{Z})} e\left(\frac{1}{2} \operatorname{tr}\left(X^{T} Q^{-1} X Z\right)\right) \sum_{V} e\left(\frac{1}{2} \operatorname{tr}\left(V^{T} Q^{-1} V S+2 V^{T} Q^{-1} X\right)\right) .
\end{aligned}
$$

where the $V$ sum runs over $V \in M_{2 k, 2}(\mathbb{Z}) / Q M_{2 k, 2}(\mathbb{Z})$. Hence, we obtain that

$$
\begin{aligned}
\theta(Q, Z) \mid\left[-\tau_{M} J \tau_{S} J \tau_{K}\right] & =(\operatorname{det} Q)^{-2} \sum_{X \in M_{2 k, 2}(\mathbb{Z})} e\left(\frac{1}{2} \operatorname{tr}\left(X^{T} Q^{-1} X(Z+K)\right)\right. \\
& \times \sum_{V \in M_{2 k, 2}(\mathbb{Z}) / Q M_{2 k, 2}(\mathbb{Z})} e\left(\frac{1}{2} \operatorname{tr}\left(V^{T} Q^{-1} V S+2 V^{T} Q^{-1} X\right)\right) .
\end{aligned}
$$

We set $X=\left(x_{1}, x_{2}\right)$ and $V=\left(v_{1}, v_{2}\right)$ and start with the $\iota_{1}(J)$ case:

$$
\begin{aligned}
& \theta(Q, Z) \mid\left[\iota_{1}(J)\right]=(\operatorname{det} Q)^{-2} \sum_{x_{1}, x_{2} \in \mathbb{Z}^{2 k}} e\left(\frac{1}{2}\left(x_{1}^{T} Q^{-1} x_{1} z_{1}+2 x_{1}^{T} Q^{-1} x_{2} z_{2}+x_{2}^{T} Q^{-1} x_{2} z_{4}\right)\right) \\
& \times e\left(-\frac{1}{2} x_{1}^{T} Q^{-1} x_{1}\right) \sum_{v_{1} \in \mathbb{Z}^{2 k} / Q \mathbb{Z}^{2 k}} e\left(-\frac{1}{2} v_{1}^{T} Q^{-1} v_{1}+v_{1}^{T} Q^{-1} x_{1}\right) \sum_{v_{2} \in \mathbb{Z}^{2 k} / Q \mathbb{Z}^{2 k}} e\left(v_{2}^{T} Q^{-1} x_{2}\right) .
\end{aligned}
$$

By the orthogonality relation, the last sum vanishes unless $x_{2} \equiv 0\left(\bmod Q \mathbb{Z}^{2 k}\right)$ and is of size $\operatorname{det} Q$ in this case. For the $v_{1}$ sum we apply the bound for symplectic Gauss sums from [57, Theorem 1] which states that

$$
\sum_{v \in \mathbb{Z}^{2 k} / Q \mathbb{Z}^{2 k}} e\left(-\frac{1}{2} v^{T} Q^{-1} v\right)=\zeta(\operatorname{det} Q)^{1 / 2}
$$

for a fourth root of unity $\zeta$. This gives the first statement.

For the second part, we obtain

$$
\begin{aligned}
& \theta(Q, Z) \mid\left[\iota_{2}(J)\right]=(\operatorname{det} Q)^{-2} \sum_{x_{1}, x_{2} \in \mathbb{Z}^{2 k}} e\left(\frac{1}{2}\left(x_{1}^{T} Q^{-1} x_{1} z_{1}+2 x_{1}^{T} Q^{-1} x_{2} z_{2}+x_{2}^{T} Q^{-1} x_{2} z_{4}\right)\right) \\
& \times e\left(-\frac{1}{2} x_{2}^{T} Q^{-1} x_{2}\right) \sum_{v_{2} \in \mathbb{Z}^{2 k} / Q \mathbb{Z}^{2 k}} e\left(-\frac{1}{2} v_{2}^{T} Q^{-1} v_{2}+v_{2}^{T} Q^{-1} x_{2}\right) \sum_{v_{1} \in \mathbb{Z}^{2 k} / Q \mathbb{Z}^{2 k}} e\left(v_{1}^{T} Q^{-1} x_{1}\right) .
\end{aligned}
$$

Similarly, the last sum vanishes unless $x_{1} \equiv 0\left(\bmod Q \mathbb{Z}^{2 k}\right)$ and we apply the previous estimate for symplectic Gauss sums. 
If

$$
\theta(Q, Z) \mid\left[\iota_{1}(J)\right]=\sum_{T \in \mathscr{S}} R_{1}(T) e\left(\frac{\operatorname{tr}(T Z)}{N}\right)
$$

with $t_{2}, t_{4} \equiv 0(\bmod N)$, then, we have by the result above that

$\left|R_{1}(T)\right|=(\operatorname{det} Q)^{-\frac{1}{2}} \#\left\{x_{1}, x_{2} \in \mathbb{Z}^{2 k} \mid t_{1}=x_{1}^{T} N Q^{-1} x_{1}, t_{2}=N x_{1}^{T} x_{2}, t_{4}=N x_{2}^{T} Q x_{2}\right\}$.

Again, we count solutions $x_{1}, y_{1}$ and $x_{2}, y_{2}$ such that $t_{1}=x_{1}^{T} N Q^{-1} x_{1}=y_{1}^{T} N Q^{-1} y_{1}$ and $t_{4}=N x_{2}^{T} Q x_{2}=N y_{2}^{T} Q y_{2}$. This automatically determines $t_{2}$ and we drop the condition that $x_{1}^{T} x_{2}=y_{1}^{T} y_{2}$. Hence, the theta series contributes in the $R(1)$ case at most

$$
\begin{aligned}
& N \sum_{\substack{T \in \mathscr{S}, t_{1}, t_{2}, t_{4} \ll N^{1+\epsilon} \\
t_{2}, t_{4} \equiv 0(\bmod N)}} \frac{r\left(N Q^{-1}, t_{1}\right)^{2} r\left(N Q, t_{4}\right)^{2}}{\operatorname{det} Q}\left(\frac{N^{2}}{\operatorname{det} T}\right)^{k-\frac{3}{2}} \\
& \ll N \sum_{\substack{t_{1} t_{4}-N\left(t_{2} / 2\right)^{2}>0 \\
t_{1} \ll N^{1+\epsilon} ; t_{2}, t_{4} \ll N^{\epsilon}}} \frac{r\left(N Q^{-1}, t_{1}\right)^{2}}{\operatorname{det} Q} r\left(Q, t_{4}\right)\left(\frac{N}{t_{1} t_{4}-N t_{2}^{2} / 4}\right)^{k-\frac{3}{2}} \ll \frac{N^{k-\frac{1}{2}+\epsilon}}{\sqrt{a}} .
\end{aligned}
$$

For the last step, we trivially estimate $\left(t_{1} t_{4}-N\left(t_{2} / 2\right)^{2} \geqslant 1\right.$ and apply Lemam 22 for the $t_{1}$-sum. The estimation of the genus theta series works similarly and by (61) we obtain a contribution of size $N^{k-\frac{3}{2}+\epsilon}$.

The $R(2)$ case proves to be more challenging. We set

$$
\theta(Q, Z) \mid\left[\iota_{2}(J)\right]=\sum_{\in \mathscr{S}} R_{2}(T) e\left(\frac{\operatorname{tr}(T Z)}{N}\right) .
$$

By Lemma 39 we need to estimate

$$
\sum_{\substack{T \in \mathscr{S}, t_{1}, t_{2} \equiv 0(\bmod N) \\ t_{1}, t_{2}, t_{4} \ll N^{1+\epsilon}}} \sum_{U}\left|R_{2}\left(U T U^{T}\right)\right|^{2}\left(\frac{N^{2}}{\operatorname{det} T}\right)^{k-\frac{3}{2}}
$$

where $U$ runs over $U=\left(\begin{array}{ll}1 & u \\ & 1\end{array}\right)$ with $0 \leqslant u \leqslant N-1$. The main obstacle lies in the fact that we need to estimate the Fourier coefficients at $U T U^{T}$. Since $G \mid\left[\iota_{2}(J)\right](Z)$ transforms with respect to 88 we cannot assume $A\left(U T U^{T}\right)=A(T)$. Furthermore, for

$$
U T U^{T}=\left(\begin{array}{cc}
\tilde{t_{1}} & \tilde{t}_{2} / 2 \\
\tilde{t}_{2} / 2 & \tilde{t}_{4}
\end{array}\right)
$$


and $u>0$ the growth and congruence conditions for $T$ transform into

$$
\tilde{t}_{1} \ll u^{2} N^{1+\epsilon}, \tilde{t}_{2} \ll u N^{1+\epsilon} \text { and } N\left|\tilde{t}_{1}+u \tilde{t}_{2}, N\right| t_{2}+u t_{4} .
$$

A way out is to bound $R_{2}(T)$ by a Fourier coefficient that satisfies $A(T)=$ $A\left(U^{T} T U\right)$ for $U \in \mathrm{SL}_{2}(\mathbb{Z})$. This way, we can assume that $T$ is Minkowski-reduced. By Lemma 62 we have that

$$
\theta \mid\left[\iota_{2}(J)\right](Z)=\sum_{X \in \mathbb{Z}^{2 k}, x_{1} \equiv 0\left(\bmod Q \mathbb{Z}^{2 k}\right)} \beta(X, Q) e\left(\frac{\operatorname{tr}\left(\frac{1}{2} X^{T} N Q^{-1} X Z\right)}{N}\right)
$$

with $|\beta(X, Q)|=(\operatorname{det} Q)^{-\frac{1}{2}}$. We drop the condition $x_{1} \equiv 0\left(\bmod Q \mathbb{Z}^{2 k}\right)$ and estimate

$$
R_{2}(T) \leqslant \frac{r\left(N Q^{-1}, T\right)}{\sqrt{\operatorname{det} Q}}
$$

By doing so, the $R(2)$ contribution is bounded by

$$
N^{2} \sum_{T \in \mathscr{T}} \frac{r\left(N Q^{-1}, T\right)^{2}}{\operatorname{det} Q}\left(\frac{N^{2}}{\operatorname{det} T}\right)^{k-\frac{3}{2}}
$$

where $\mathscr{T} \subset\left\{T \in \mathcal{R}_{2}, N \mid \operatorname{det} T\right\}$ with $\# \mathscr{T} \ll N^{1+\epsilon}$. This term is bounded by

$$
N^{2} \sum_{\substack{N \ll t_{1} t_{4} \ll N^{2+\epsilon} \\ \# t_{1} t_{4} \ll N^{1+\epsilon}}} \frac{r\left(N Q^{-1}, t_{1}\right)^{2} r\left(N Q^{-1}, t_{4}\right)^{2}}{\operatorname{det} Q}\left(\frac{N^{2}}{t_{1} t_{4}}\right)^{k-\frac{3}{2}} .
$$

To estimate $r\left(N Q^{-1}, t_{1}\right)^{2}$ and $r\left(N Q^{-1}, t_{4}\right)^{2}$ we apply 65 which gives

$$
r\left(N Q^{-1}, t\right)^{2} \ll\left(1+\ldots+\frac{t^{2 k-2} \operatorname{det} Q}{N^{2 k-2} a}\right) N^{\epsilon} .
$$

Again, the largest contribution comes from the case when $t_{1}$ is small and $t_{4}=N^{2+\epsilon}$. As a consequence, display 103 is bounded by

$$
\frac{N^{2 k-1}}{\operatorname{det} Q} \sum_{\substack{t_{4} \ll N^{2+\epsilon} \\ \# t_{4} \ll N^{1+\epsilon}}}\left(1+\ldots+\frac{t_{4}^{k-\frac{1}{2}} \operatorname{det} Q}{N^{2 k-2} a}\right) N^{\epsilon} \ll \frac{N^{2 k+1}}{a} N^{\epsilon} .
$$

The bounds for the genus theta series are significantly stronger and by proceeding as above and applying (61), we obtain a contribution of size $\operatorname{det} Q$ for the genus theta series in the $R(2)$ case.

As a consequence, we obtain: 
Proposition 63. Let $G(Z)=\theta(Q, Z)-\theta($ gen $Q, Z)-E\left(f_{1}, Z\right)-E\left(f_{2}, Z\right)$. Then

$$
\langle G, G\rangle \ll \frac{N^{2 k+1}}{a} N^{\epsilon} .
$$

Proof. As previously seen, it holds that

$$
\langle G, G\rangle=\left.\sum_{i=0}^{3} \sum_{\gamma \in R(i)} \int_{F}|G|[\gamma](Z)\right|^{2}(\operatorname{det} Y)^{3} d X \frac{D Y}{(\operatorname{det} Y)^{k}} .
$$

Let $g(T), g_{1}(T), g_{2}(T), g_{3}(T)$ denote the Fourier coefficients of $G, G \mid\left[\iota_{1}(J)\right], G\left[\iota_{2}(J)\right]$ and $G \mid\left[J_{4}\right]$. Then, we have by Lemma 37,38 and 39 that

$$
\begin{aligned}
\langle G, G\rangle & \ll \sum_{\substack{T \in \mathscr{S} \\
t_{1},\left|t_{2}\right|, t_{4} \ll 1}}|g(T)|^{2}+N^{2 k-2} \sum_{T \in \mathscr{S}} \frac{\left|g_{1}(T)\right|^{2}}{(\operatorname{det} T)^{k-\frac{3}{2}}} \\
& +N^{2 k-2} \sum_{u=0}^{N-1} \sum_{T \in \mathscr{S}} \frac{\mid g_{2}\left(\left.U T U^{T}\right|^{2}\right.}{(\operatorname{det} T)^{k-\frac{3}{2}}}+N^{2 k} \sum_{\substack{t_{1}, t_{2}, t_{4} \ll N^{1+\epsilon} \\
\in \mathscr{S}}} \frac{\left|g_{3}(T)\right|^{2}}{(\operatorname{det} T)^{k-\frac{3}{2}}},
\end{aligned}
$$

where $U=\left(\begin{array}{ll}1 & u \\ & 1\end{array}\right)$. We bound the Fourier coefficients by

$$
|g(T)|^{2} \ll|A(T)|^{2}+|B(T)|^{2}+r(Q, T)^{2}+r(\operatorname{gen} Q, T)^{2}
$$

where $A(T), B(T)$ are the Fourier coefficients of $E\left(f_{1}, Z\right)$ and $E_{N}\left(f_{2}, Z\right)$. This gives a bound of size (96) for the first term in display (105). Analogously, we bound $\left|g_{1}(T)\right|^{2},\left|g_{2}(T)\right|^{2},\left|g_{3}(T)\right|^{2}$. The resulting bound for the contribution of $R(1)$ is given in (98) and (102), of $R(2)$ in $(99)$ and $(104)$ and of $R(3)$ in (97) and (101) The bound induced by the theta series in the $R(2)$ case dominates all other error terms.

There is certainly still room for improvement. For Klingen-Eisenstein series the main obstacle is to transform the negative power in $\min T$ into a considerable saving with respect to $N$. For the theta series, we run into difficulties when estimating Fourier coefficients for which both, $t_{1}$ and $t_{4}$ are large but $\operatorname{det} T$ is small. A way to address this issue is to assume that $T$ is Minkowski-reduced. The downside is, however, that growth and congruence conditions for the entries of $T$ are altered.

By (80), Proposition 63 gives the following upper bound for the Fourier coefficients of $G$ :

$$
g(T) \ll \frac{N^{k}}{a}\left((\operatorname{det} T)^{\frac{k}{2}-\frac{1}{4}+\epsilon}+N^{\frac{1}{2}}\right) N^{\epsilon} .
$$


As a consequence of Theorem (56) and the display above, we obtain $r(Q, T)=$ $r($ gen $Q, T)+E(Q, T)$ with

$$
\begin{aligned}
& E(Q, T) \ll(\operatorname{det} T)^{k-\frac{3}{2}}(\min T)^{\frac{1-k}{2}}\left(\frac{N^{\frac{k}{2}}}{\operatorname{det} Q}+\frac{N^{\frac{1}{2}}}{\sqrt{\operatorname{det} Q} \sqrt[4]{a}}+\left(\frac{N^{\frac{3-k}{2}}}{\sqrt{\operatorname{det} Q}}+\frac{N^{-k+2}}{\sqrt[4]{a}}\right)(N, T)^{\frac{1}{2}}\right) \\
& \left.\times\left(1+\frac{(\min T)^{\frac{1}{4}}(\min T, N)^{\frac{1}{4}}}{N^{\frac{1}{2}}}\right)+\left(\left(1+\frac{(\min T)^{\frac{1}{4}}}{N^{\frac{1}{2}}}\right) \frac{N^{\frac{1-k}{2}+\epsilon} \sqrt[4]{\tilde{a}}}{\sqrt{\operatorname{det} Q}}(N, \operatorname{det} T) \delta_{N \mid \min T}\right) N^{\epsilon}\right) \\
& +(\operatorname{det} T)^{\frac{3}{4} k-\frac{5}{4}}\left(\frac{N^{2-\frac{k}{2}}}{\sqrt{\operatorname{det} Q}}+\frac{N^{-k+\frac{5}{2}}}{\sqrt[4]{a}}+\frac{N^{\frac{k}{2}}}{\operatorname{det} Q}+\frac{N^{\frac{1}{2}}}{\sqrt{\operatorname{det} Q} \sqrt[4]{a}}\right)\left(1+\frac{(\operatorname{det} T)^{\frac{1}{8}}}{N^{\frac{1}{4}}}\right) N^{\epsilon} \\
& +(\operatorname{det} T)^{\frac{k}{2}-\frac{1}{4}+\epsilon} \frac{N^{k+\epsilon}}{a}+\frac{N^{k+\frac{1}{2}+\epsilon}}{a} .
\end{aligned}
$$

Together with Corollary 35, this gives the following result:

Theorem 64. Let $k \geqslant 6$ be even. Consider a positive, integral $2 k \times 2 k$ matrix $Q$ of large prime level $N$ such that $\operatorname{det} Q$ is a square. A binary quadratic form $T>0$ is represented by $Q$, i.e. $T=X^{T} Q X$ is soluble for $X \in \mathbb{Z}^{2 k \times 2}$, if $N^{2 k-4} \nmid \operatorname{det} Q$ and

$$
\begin{aligned}
& \min T \gg N^{1+\frac{2}{2 k-3}+\epsilon}+\left(\frac{\sqrt{N} \operatorname{det} Q}{\sqrt{a}}\right)^{\frac{2}{2 k-3}} N^{\epsilon}, \\
& \operatorname{det} T \gg N^{2+\epsilon}\left(\frac{N^{5}(\operatorname{det} Q)^{2}}{a}\right)^{\frac{2}{2 k-5}} .
\end{aligned}
$$

Remark. If $\operatorname{det} Q \leqslant N^{k-\frac{1}{2}}$ only the first term in each condition is relevant. For $N^{2 k-4} \mid \operatorname{det} Q$ and $(\operatorname{det} T, N)=1$ we obtain the same result with the additional condition that

$$
\min T \gg\left(\frac{N^{-2 k+\frac{7}{2}}(\operatorname{det} Q)^{2}(N, T)}{\sqrt{a}}\right)^{\frac{2}{2 k-3}} N^{\epsilon} .
$$

If $(N, \min T)=1$, the exponent in the lower bound of $\min T$ improves to $\frac{1}{k-1}$ instead of $\frac{2}{2 k-3}$.

\subsection{Extensions}

We discuss two ways to extend the results in Theorem 6. The aim of the main part is the treatment of the squarefree level case. In the second part we discuss how to drop the requirement in Theorem 6 that the determinant is a square. This involves the estimation of Eisenstein series and cusp forms transforming with respect to quadratic characters. 


\subsubsection{Squarefree level}

We explain how to extend the results for Klingen-Eisenstein series and Siegel theta series to squarefree level. We state the necessary theoretical framework but keep the proofs very short. The structure follows the prime level case.

To start, we recall the setting of Section 4.1. For squarefree level we stated the following basis of the Klingen-Eisenstein space:

$$
E_{l}(f, Z)=l^{k} \sum_{M \in P(N) \backslash \Gamma_{0}(N) \gamma(l)} j(M, l Z) f(\pi(M(l Z)))
$$

where $l \mid N, f \in S_{k}(N)$, and $\gamma_{l}$ satisfies

$$
\operatorname{Sp}_{4}(\mathbb{Z}) \ni \gamma(l) \equiv\left\{\begin{array}{l}
J_{4} \bmod l^{2} \\
I_{4} \bmod (N / l)^{2}
\end{array}\right.
$$

We let $\gamma_{1}(l)$ denote the corresponding element in $\mathrm{SL}_{2}(\mathbb{Z})$, i.e., $\gamma_{1}(l) \equiv J(\bmod l)$ and $\gamma_{1}(l) \equiv I(\bmod N / l)$. For simplicity, we assume that $\gamma(l)$ has the form

$$
\gamma(l)=\iota_{1}(\gamma(l)) \iota_{2}(\gamma(l))
$$

Furthermore, we set

$$
\kappa(l)=\gamma(l)\left(\begin{array}{ll}
l I & \\
& I
\end{array}\right) \quad \text { and } \quad \kappa_{1}(l)=\gamma_{1}(l)\left(\begin{array}{ll}
l & \\
& 1
\end{array}\right) .
$$

By [9, p. 127] we have for $l|N, m| N, d=(l, m), l=\tilde{l} d, m=\tilde{m} d$ that

$$
\Gamma_{0}^{(2)}(N) \kappa(l) \kappa(m)=\Gamma_{0}^{(2)}(N) \kappa(\tilde{l} \tilde{m})\left(\begin{array}{ll}
d I & \\
& d I
\end{array}\right)
$$

We decompose

$$
\theta(Q, Z)=\theta(\operatorname{gen} Q, Z)+\sum_{l \mid N} E_{l}\left(f_{l}, Z\right)+G(Z)
$$

for a cusp form $G(Z)$. An application of the Siegel $\Phi$-operator yields that $f_{l}(z)$ is given by $\Phi_{l}(\theta(Q, *)-\theta($ gen $Q, *))(z)$, where

$$
\Phi_{l}(\theta(Q, *))(z)=\lim _{t \rightarrow \infty}(\theta(Q, *) \mid[\kappa(l)])\left(\begin{array}{ll}
z & \\
& i t
\end{array}\right) .
$$

The computation of $f_{l}$ proves to be more challenging than in the prime level case. To this end, we recall the results for the one-dimensional theta series. By Lemma 23 it holds for $l \mid N$ with $l \neq 1$ that

$$
\theta(Q, z) \mid\left[\gamma_{1}(l)\right]=\frac{1}{\sqrt{\left(\operatorname{det} Q, l^{2 k}\right)}} \sum_{x \in \mathbb{Z}^{2 k}} \alpha(x, Q, l) e\left(\frac{1}{2} x^{T} D_{l}\left(V^{T} Q V\right)^{-1} D_{l} x z\right),
$$


where $\left(V^{T} Q^{-1} V D_{l}\right)^{-1}$ is an integer matrix of determinant $\left(\operatorname{det} Q, l^{2 k}\right)$ and level l. Furthermore, $|\alpha(x, Q, l)|=1$ and $D_{l}$ is a diagonal matrix of determinant $\left(\operatorname{det} Q,(N / l)^{2 k}\right){ }_{m}^{11}$ From now on, we fix $V$ such that $V^{T} Q V D_{m}^{-1}$ is an integral matrix of level $m$ for all $m \mid N$.

We establish a similar transformation formula for the theta series of degree two:

Lemma 65. Let $1 \neq l$ be a divisor of $N$. Then, it holds that

$$
\theta(Q, Z) \mid\left[\gamma_{l}\right]=\frac{1}{\left(\operatorname{det} Q, l^{2 k}\right)} \sum_{X \in M_{2 k, 2}(\mathbb{Z})} \alpha(X, Q, l) e\left(\operatorname{tr}\left(X^{T} D_{l}\left(V^{T} Q V\right)^{-1} D_{l} X Z\right)\right)
$$

where $|\alpha(x, Q, l)|=1$ and $D_{l}$ and $V$ satisfy the same properties as above. Furthermore, we have that

$$
\Phi(\theta(Q, *) \mid[\kappa(l)])(z)=\zeta \frac{l^{\frac{k}{2}}}{\sqrt{\left(\operatorname{det} Q, l^{2 k}\right)}} \theta(Q, z) \mid\left[\kappa_{1}(l)\right] .
$$

for some root of unity $\zeta$.

Proof. Let

$$
\gamma(l)=\left(\begin{array}{llll}
a & 0 & * & 0 \\
0 & a & 0 & * \\
c & 0 & d & 0 \\
0 & c & 0 & d
\end{array}\right)
$$

with $a \equiv 1, c \equiv 0\left(\bmod (N / l)^{2}\right), a \equiv 0, c \equiv 1\left(\bmod l^{2}\right)$. By Lemma 31 we have that

$$
\theta(Q, Z) \mid[\gamma(l)]=\sum_{X \in M_{2 k, 2}(\mathbb{Z})} \alpha(X, Q, l) e\left(\frac{1}{2} X^{T} Q^{-1} X Z\right)
$$

with $X=\left(x_{1}, x_{2}\right) \in M_{2 k, 2}(\mathbb{Z})$ and

$$
\begin{aligned}
\alpha(X, Q, l) & =\frac{\omega}{c^{2 k} \operatorname{det} Q} e\left(\frac{d}{2 c}\left(x_{1}^{T} Q^{-1} x_{1}+x_{2}^{T} Q^{-1} x_{2}\right)\right) \\
& \times \sum_{v_{1}, v_{2} \in \mathbb{Z}^{2 k} / c \mathbb{Z}^{2 k}} e\left(\frac{a}{2 c}\left(v_{1}^{T} Q v_{1}+v_{2}^{T} Q v_{2}\right)\right) e\left(\frac{1}{c}\left(x_{1}^{T} v_{1}+x_{2}^{T} v_{2}\right)\right),
\end{aligned}
$$

where $\omega \in\{ \pm 1\}$. This reduces the problem to the one-dimensional case which we already computed in Lemma 23.

\footnotetext{
${ }^{11}$ Note that this part uses a slightly different notation compared to Lemma 23 . The lower left entry $c$ of $\gamma_{1}(l)$ satisfies $(c, N)=\frac{N}{l}$. This means $\left(V^{T} Q V\right)^{-1} D_{l}$ corresponds to $Q_{N / l}$ in Lemma 23
} 
To prove the second claim, let $X=\left(x_{1}, x_{2}\right) \in M_{2 k, 2}(\mathbb{Z})$. If we apply the Siegel $\Phi$-operator on

$$
\frac{1}{\left(\operatorname{det} Q, l^{2 k}\right)} \sum_{X \in M_{2 k, 2}(\mathbb{Z})} \alpha(X, Q, l) e\left(\operatorname{tr}\left(X^{T} D_{l}\left(V^{T} Q V\right)^{-1} D_{l} X Z\right)\right)
$$

all terms with $x_{2} \neq 0$ vanish. If $x_{2} \neq 0$, we have

$$
\begin{aligned}
\alpha(X, Q, l) & =\frac{\omega}{c^{2 k} \operatorname{det} Q} e\left(\frac{d}{2 c} x_{1}^{T} Q^{-1} x_{1}\right) \\
& \times \sum_{v_{1} \in \mathbb{Z}^{2 k} / c \mathbb{Z}^{2 k}} e\left(\frac{a}{2 c} v_{1}^{T} Q v_{1}+\frac{1}{c} x_{1}^{T} v_{1}\right) \sum_{v_{2} \in \mathbb{Z}^{2 k} / c \mathbb{Z}^{2 k}} e\left(\frac{a}{2 c} v_{2}^{T} Q v_{2}\right) .
\end{aligned}
$$

Since $(c, N)=\frac{N}{l}$ the Gauss sum equals

$$
\sum_{v_{2} \in \mathbb{Z}^{2 k} / c \mathbb{Z}^{2 k}} e\left(\frac{a}{2 c}\left(v_{2}^{T} Q v_{2}\right)\right)=\xi c^{k} \sqrt{\left(\operatorname{det} Q,(N / l)^{2 k}\right)}
$$

for some root of unity $\xi$. Thus, for $x_{2}=0$ display (107) equals

$$
\frac{\xi \omega l^{\frac{k}{2}}}{c^{k} \sqrt{\left(\operatorname{det} Q, l^{2 k}\right)} \sqrt{\operatorname{det} Q}} e\left(\frac{d}{2 c}\left(x_{1}^{T} Q^{-1} x_{1}\right) \sum_{v_{1} \in \mathbb{Z}^{2 k} / c \mathbb{Z}^{2 k}} e\left(\frac{a}{2 c} v_{1}^{T} Q v_{1}+\frac{1}{c} x_{1}^{T} v_{1}\right) .\right.
$$

Note that both $\xi$ and $\omega=(-1)^{k}$ are independent of $x_{1}$. Furthermore, the term $\zeta$ in Lemma 23 equals $i^{k}$ and hence, also does not depend on $x_{1}$. The second claim now follows by comparing the term above with the display below (66).

By 106$)$ it holds that

$$
\Gamma_{0}^{(2)}(N) \kappa(l)^{-1}=\Gamma_{0}^{(2)}(N) \kappa(l)\left(\begin{array}{ll}
l I & \\
& l I
\end{array}\right) .
$$

Hence, we obtain by the lemma above that

$$
f_{l}(z)=\zeta \frac{l^{\frac{k}{2}}}{\sqrt{\left(\operatorname{det} Q, l^{2 k}\right)}}\left(\theta(Q, z)\left|\left[\kappa_{1}(l)\right]-\theta(\operatorname{gen} Q, z)\right|\left[\kappa_{1}(l)\right]\right) .
$$

To bound the Fourier coefficients of $E_{l}\left(f_{l}, Z\right)$, we need to bound the inner product of $f_{l} \mid\left[\kappa_{1}(m)\right]$ for $m \mid N$. For this purpose, we set $S_{l}=l D_{l}\left(V^{T} Q V\right)^{-1} D_{l}$ and

$$
a(l)= \begin{cases}a_{2 k-1} a_{2 k} & \text { if } \frac{1}{2} x^{T} S_{l} x=\sum_{i} a_{i} x_{i}^{2} \text { with } a_{i} \leqslant a_{i+1} \\ \left(\operatorname{det} S_{l}\right)^{\frac{1}{k}} & \text { otherwise }\end{cases}
$$


Note that $\operatorname{det} S_{l}=l^{2 k} \frac{\left(\operatorname{det} Q,(N / l)^{2 k}\right)^{2}}{\operatorname{det} Q}$. To apply Lemma 24 we need to compute $\left(\operatorname{det} S_{l}, N^{k}\right)$.

To hold notation simple, we assume for the rest of this section that $\left(\operatorname{det} Q, N^{k}\right)=$ $\operatorname{det} Q$. The remaining cases can be treated in a similar manner. With this assumption, we obtain for $m=1$ that

$$
\left\langle f_{l}, f_{l}\right\rangle \ll \frac{l^{k}}{\left(\operatorname{det} Q, l^{k}\right)}\left(\frac{N^{k}}{\operatorname{det} Q}+\frac{l^{k} N}{\left(\operatorname{det} Q, l^{k}\right) \sqrt{a(l)}}\right) .
$$

For $m>1$ we estimate the Petersson inner product as follows:

Corollary 66. Let $d=(m, l)$ and $l=\tilde{l} d, m=\tilde{m} d$. Then,

$$
\left\langle f_{l}\left|\left[\left(\kappa_{1}(m)\right)\right](z), f_{l}\right|\left[\left(\kappa_{1}(m)\right)\right](z)\right\rangle \ll \frac{l^{k}}{\left(\operatorname{det} Q, l^{k}\right)}\left(\frac{N^{k}}{\operatorname{det} Q}+\frac{\tilde{l}^{k} \tilde{m}^{k} N}{\left(\operatorname{det} Q,(\tilde{l} \tilde{m})^{k}\right) \sqrt{a(\tilde{l} \tilde{m})}}\right) .
$$

Proof. By (106) we have that

$$
\Gamma_{0}(N) \kappa_{1}(l) \kappa_{1}(m)=\Gamma_{0}(N) \kappa_{1}(\tilde{l} \tilde{m})\left(\begin{array}{ll}
d & \\
& d
\end{array}\right) .
$$

It follows by Lemma 23 that

$$
f_{l} \mid[\kappa(m)](z)=\frac{l^{\frac{k}{2}}}{\sqrt{\left(\operatorname{det} Q, l^{k}\right)}} \frac{(\tilde{l} \tilde{m})^{\frac{k}{2}}}{\sqrt{\left(\operatorname{det} Q,(\tilde{l} \tilde{m})^{k}\right)}}(\theta(S, z)-\theta(\operatorname{gen} S, z))
$$

for $S=\tilde{m} \tilde{l} D_{\tilde{l} \tilde{m}}\left(V^{T} Q V\right)^{-1} D_{\tilde{l} \tilde{m}}$. The matrix $S$ has level $N$ and determinant

$$
\tilde{l}^{2 k} \tilde{m}^{2 k} \frac{\left(\operatorname{det} Q,(N / \tilde{m} \tilde{l})^{k}\right)}{\left(\operatorname{det} Q,(\tilde{m} \tilde{l})^{k}\right)}
$$

By

$$
\left(\operatorname{det} S, N^{k}\right)=\frac{\tilde{l}^{k} \tilde{m}^{k} \operatorname{det} Q}{\left(\operatorname{det} Q,(\tilde{l} \tilde{m})^{k}\right)}
$$

and Lemma 24, the claim follows.

We continue as in Section 4.3.2 and 4.3.3. First, we determine a decomposition of $\mathcal{M}_{l}=\left\{M \in P(N) \backslash \Gamma_{0}(N) \gamma_{l} \mid \lambda(M) \neq(0,0, *, *)\right\}$ :

Lemma 67. There is a unique decomposition

$$
\mathcal{M}_{l}=\bigsqcup_{r s=\frac{N}{l}} \bigsqcup_{\sigma, v, w, \mu} P(N) L\left(\begin{array}{ll}
v & \\
& v^{-T}
\end{array}\right) \iota_{2}(\sigma)\left(\begin{array}{cc}
I & \mu \\
& I
\end{array}\right)\left(\begin{array}{ll}
w^{-1} & \\
& w^{T}
\end{array}\right)
$$


for

$$
\begin{aligned}
\mu & =\left(\begin{array}{cc}
0 & \mu_{2} \\
\mu_{2} & \mu_{4}
\end{array}\right) \text { and } \mu_{2}, \mu_{4} \in l \mathbb{Z}, \\
\sigma & =\left(\begin{array}{ll}
* & * \\
c & d
\end{array}\right) \in \Gamma_{\infty} \backslash \mathrm{SL}_{2}(\mathbb{Z}) / \Gamma_{\infty}(l), c>0 \\
v & =\left(\begin{array}{ll}
v_{1} & v_{2} \\
* & *
\end{array}\right) \in \Gamma_{\infty}^{T} \backslash \mathrm{SL}_{2}(\mathbb{Z}) / \Gamma_{\infty}(l c), v_{1}>0, \\
w & =\left(\begin{array}{ll}
w_{1} & w_{2} \\
w_{3} & w_{4}
\end{array}\right) \in \operatorname{PSL}_{2}(\mathbb{Z}) / \bar{\Gamma}_{\infty}
\end{aligned}
$$

with $c \equiv 0(\bmod r), c \not \equiv 0(\bmod s), d \equiv 0(\bmod l), v_{1} \equiv 0(\bmod s), v_{2} \equiv 0(\bmod l)$ and

$$
L=\iota_{1}\left(\gamma_{1}(l s)\left(\begin{array}{cc}
1 & l r d_{1} \\
& 1
\end{array}\right)\right) \in \mathrm{P}(\mathbb{Z})
$$

with $d_{1} \equiv a v_{2} \overline{c v_{3} l r}(\bmod s)$.

Proof. By Corollary 45 it is sufficient to consider

$$
\bigsqcup_{\sigma, v, w, \mu} P(N) L\left(\begin{array}{ll}
v & \\
& v^{-T}
\end{array}\right) \iota_{2}(\sigma)\left(\begin{array}{cc}
I & \mu \\
& I
\end{array}\right)\left(\begin{array}{ll}
w^{-1} & \\
& w^{T}
\end{array}\right) \gamma(l)^{-1}
$$

and to determine congruence conditions such that all elements in the decomposition above are contained in $\Gamma_{0}^{(2)}(N)$. As $N$ is squarefree, we may split $N=\operatorname{lm}$ and consider these cases separately.

Modulo $l$ we proceed as in Lemma 47 and obtain that $l$ divides both $d, v_{2}$ and that

$$
\omega(L) \equiv\left(\begin{array}{ll} 
& -1 \\
1 &
\end{array}\right)(\bmod l)
$$

For $m=\frac{N}{l}$ we follow the argument in Lemma 46 which yields that $c v \equiv 0(\bmod m)$. We decompose $m=r s$ such that $c \equiv 0(\bmod r), c \neq \equiv 0(\bmod s)$ and $v_{1} \equiv 0(\bmod s)$. Furthermore, we have by Lemma 46 that

$$
\omega(L) \equiv I(\bmod r) \text { and } \omega(L) \equiv\left(\begin{array}{cc} 
& -1 \\
1 & d_{1}
\end{array}\right)(\bmod s)
$$

where $d_{1} \equiv-a v_{2}^{2} \bar{c}(\bmod s)$. The choice above for $L$ satisfies all three congruences.

Next, we determine the Fourier coefficients $A_{l}(T)$ of $E_{l}\left(f_{l}, Z\right)=\sum_{T} A_{l}(T) e(\operatorname{tr} T Z)$. To this end, we proceed as in Section 4.3.3. 
Corollary 68. It holds that

$$
\begin{aligned}
& A_{l}(T)=C_{k}(\operatorname{det} T)^{k-\frac{3}{2}} \sum_{r s=\frac{N}{l}} \sum_{v_{1}>0} \sum_{\substack{\left(w_{1}, w_{3}\right)=1, w_{3} \geqslant 0 \\
v_{1}^{2} s \mid T[w]_{1}}}\left(T[w]_{1}\right)^{1-k} a\left(\frac{T[w]_{1}}{s v_{1}^{2}}\right) \sum_{\substack{c>0, s \nmid c}}(r c)^{-k} \\
& \times \sum_{\substack{d \neq 0 \bmod r c \\
\text { ald } \equiv 1 \bmod r c\left(s v_{1}, l v_{2}\right)=1}} \sum_{v_{2} \bmod c s v_{1}} e\left(\frac{s^{-1} v_{1}^{-2} T[w]_{1} a l v_{2}^{2}}{s r c}-\frac{s^{-1} v_{1}^{-2} T[w]_{1} a l v_{2}^{2} \overline{r c}}{s}+\frac{T[w]_{2} v_{2}}{s v_{1} r c}+\frac{T[w]_{4} d}{r c}\right)
\end{aligned}
$$

where $a(n)$ are the Fourier coefficients of $(l s)^{-\frac{k}{2}} f_{l} \mid\left[\kappa_{1}(l s)\right]=\sum_{n \geqslant 1} a(n) e(n z)$

Note that we already substituted $v_{1}$ by $s v_{1}, c$ by $r c, d$ by $l d$ and $v_{2}$ by $l v_{2}$ in the formula above.

Proof. We apply the decomposition from Lemma 67 and follow the line of argument in Section 4.3 .3 very closely. Note that

$$
f_{l} \mid\left[\gamma_{1}(l s)\right]=\sum_{n \geqslant 1} a(n) e\left(\frac{n z}{l s}\right) e\left(\frac{n l r d_{1}}{l s}\right) .
$$

Furthermore, the $x_{1}$ integral

$$
\int_{0}^{l}\left(\frac{n v_{1}^{2}}{s}-T[w]_{1}\right) \frac{x_{1}}{l} d x_{1}
$$

vanishes unless $n=\frac{T[w]_{1} s}{v_{1}^{2}}$.

As a consequence, we obtain the following bound:

Theorem 69. For the coefficients $A_{l}(T)$ of $E_{l}(f, Z)$ we have

$$
\begin{aligned}
A_{l}(T) & \ll(\operatorname{det} T)^{k-\frac{3}{2}}(\min T)^{\frac{1-k}{2}} \sum_{r s=\frac{N}{l}} r^{\frac{3}{2}-k}(r, T)^{\frac{1}{2}} s^{\frac{1-k}{2}} \delta_{s \mid \min T}(s, \operatorname{det} T) \\
& \times(l s)^{-\frac{k}{2}}\left\|f_{l} \mid[\kappa(l s)]\right\|\left(1+\frac{(\min T)^{\frac{1}{4}}(\min T, r l)^{\frac{1}{4}}}{(r s l)^{\frac{1}{2}}}\right) \\
& +(\operatorname{det} T)^{\frac{3}{4} k-\frac{5}{4}} \sum_{r s=\frac{N}{l}} r^{2-k} s^{\frac{3}{2}-k} l^{-\frac{k}{2}}\left\|f_{l} \mid[\kappa(l s)]\right\|\left(1+\frac{(\operatorname{det} T)^{\frac{1}{8}}}{(r l)^{\frac{1}{4}} s^{\frac{1}{2}}}\right)
\end{aligned}
$$

where

$$
(l s)^{-\frac{k}{2}}\left\|f_{l} \mid[\kappa(l s)]\right\| \ll \frac{1}{\sqrt{\left(\operatorname{det} Q, l^{k}\right)}}\left(\frac{N^{\frac{k}{2}}}{s^{\frac{k}{2}} \sqrt{\operatorname{det} Q}}+\frac{\sqrt{N}}{\sqrt{\left(\operatorname{det} Q, s^{k}\right) \sqrt[4]{a(s)}}}\right) .
$$


Proof. We apply Corollary 68 and proceed as in Theorem 56. By the Petersson formula and Corollary 66 we obtain for the Fourier coefficients of $(l s)^{-\frac{k}{2}} f_{l} \mid[\kappa(l s)]=$ $\sum_{n} a(n) e(n z)$ that

$$
\begin{aligned}
a\left(\frac{T\left[w_{1}\right]}{s v_{1}^{2}}\right) & \ll\left(\frac{T\left[w_{1}\right]}{s v_{1}^{2}}\right)^{\frac{k-1}{2}}\left(1+\frac{T[w]_{1}^{\frac{1}{4}}(T[w], r l s)^{\frac{1}{4}}}{\left(s v_{1}\right)^{\frac{1}{2}}(r l)^{\frac{1}{2}}}\right) \\
& \times \frac{1}{\sqrt{\left(\operatorname{det} Q, l^{k}\right)}}\left(\frac{N^{\frac{k}{2}}}{s^{\frac{k}{2}} \sqrt{\operatorname{det} Q}}+\frac{\sqrt{N}}{\sqrt{\left(\operatorname{det} Q, s^{k}\right)} \sqrt[4]{a(s)}}\right) .
\end{aligned}
$$

Recall that $a(1)=a$ where $a$ is defined as as the product of the two largest coefficients if $Q$ is diagonal and as $(\operatorname{det} Q)^{\frac{1}{k}}$ otherwise. To obtain an asymptotic formula for $r(Q, T)$, we need to bound $\sum_{l \mid N} A_{l}(T)$.

Corollary 70. Let $A_{l}(T)$ denote the Fourier coefficients of $E_{l}\left(f_{l}, Z\right)$. Then,

$$
\sum_{l \mid N} A_{l}(T) \ll(\operatorname{det} T)^{k-\frac{3}{2}}(\min T)^{\frac{1-k}{2}}\left(\frac{N^{k / 2}}{\operatorname{det} Q}+\frac{N^{\frac{1}{2}}}{\sqrt[4]{a}}\right) .
$$

Proof. By the bound in Theorem 69 we need to find an upper bound for

$$
\sum_{l r s=N}\left(\frac{r^{2-\frac{k}{2}} s^{\frac{3}{2}-\frac{k}{2}} l^{\frac{k}{2}}}{\sqrt{\left(\operatorname{det} Q, l^{k}\right)} \sqrt{\operatorname{det} Q}}+\frac{r^{\frac{5}{2}-k} s^{2-\frac{k}{2}} l^{\frac{1}{2}}}{\sqrt{\left(\operatorname{det} Q,(s l)^{k}\right) \sqrt[4]{a(s)}}}\right) .
$$

Every prime divisor of $N$ divides either $l, r$ or $s$. The expression above is maximized when $l=N$

As in the prime case, we observe that the largest contribution derives from the Eisenstein series $E_{N}\left(f_{N}, Z\right)$ as long as the determinant is not very large compared to the level.

It remains to evaluate the Petersson inner product of

$$
G(Z)=\theta(Q, Z)-\theta(\operatorname{gen} Q, Z)-\sum_{l \mid N} f_{l}(Z)
$$

For this purpose, we state the following set of representatives:

Lemma 71. A set of right coset representatives of $\Gamma_{0}^{(2)}(N) \backslash \mathrm{Sp}_{4}(\mathbb{Z})$ for squarefree $N$ is given by

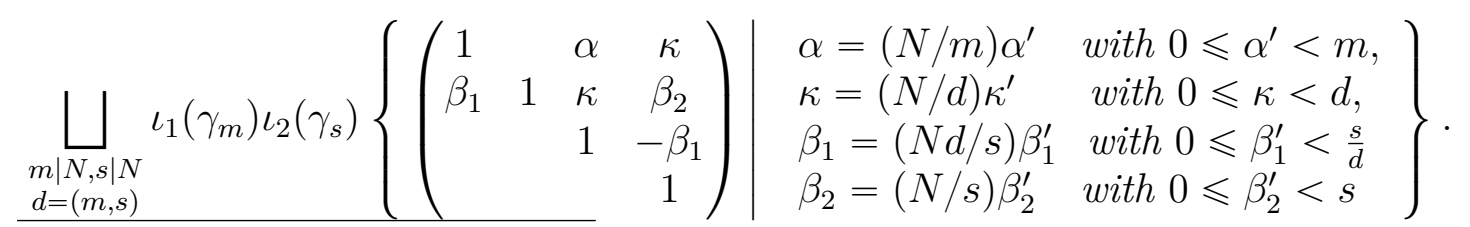

${ }^{12}$ Without the assumption $\left(\operatorname{det} Q, N^{k}\right)=\operatorname{det} Q$ it makes sense to include the congruence conditions in the cases $p \mid s$ and $p \mid r$. Therefore, we need to treat the two terms in Theorem 69 separately. 
Proof. For general $N$ a set of representatives is given in [36, Korollar 2.12]. Our set of representatives is similarly constructed as [36, Beispiel 2.14].

We set $d=(m, s)$ and $m=\tilde{m} d, s=\tilde{s} d$. Then,

$$
\begin{aligned}
\langle G, G\rangle & =\sum_{M \in \Gamma_{0}(N) \backslash \operatorname{Sp}_{4}(\mathbb{Z})} \int_{\operatorname{Sp}_{4}(\mathbb{Z}) \backslash \mathbb{H}_{2}} G \mid[M](Z)(\operatorname{det} Y)^{k-3} d X d Y \\
& =\sum_{m|N, s| N} \frac{1}{\tilde{m}^{2} \tilde{s}^{2}} \sum_{U} \int_{Y} \int_{X \bmod \tilde{m} \tilde{s} d} G \mid\left[\iota_{1}(m) \iota_{2}(s)\right]\left(U Z U^{T}\right)(\operatorname{det} Y)^{k-3} d X d Y
\end{aligned}
$$

where $U$ runs over matrices of the form $\left(\begin{array}{cc}1 & \frac{N}{\tilde{s}} \beta_{1} \\ & 1\end{array}\right)$ and $0 \leqslant \beta_{1}<\tilde{s}$. We expand

$$
G \mid\left[\iota_{1}\left(\gamma_{m}\right) \iota_{2}\left(\gamma_{s}\right)\right](Z)=\sum_{T \in \mathscr{S}} A_{m, s}(T) e\left(\frac{e(\operatorname{tr}(T Z))}{\tilde{m} d \tilde{s}}\right)
$$

where $T$ satisfies

$$
\tilde{s}\left|t_{1}, \tilde{s} \tilde{m}\right| t_{2}, \tilde{m} \mid t_{4}
$$

We insert the Fourier expansion into the integral term above and follow the chain of arguments in Lemma 38 and Lemma 39. As a consequence, we obtain:

$$
\langle G, G\rangle \ll \sum_{\substack{m|N, s| N \\ d=(m, s)}} \tilde{m} \tilde{s} d^{3} \sum_{U} \sum_{\substack{T \in \mathscr{S} \\ t_{1}, t_{2}, t_{4} \ll(\tilde{m} \tilde{s} d)^{1+\epsilon}}} A_{m, s}\left(U T U^{T}\right)\left(\frac{(\tilde{m} \tilde{s} d)^{2}}{\operatorname{det} T}\right)^{k-\frac{3}{2}} .
$$

As before, we treat the contribution of Klingen-Eisenstein and theta series separately. For the latter, the following transformation formula is useful:

Lemma 72. It holds that

$$
\begin{array}{r}
\theta(Q, Z) \mid\left[\iota_{1}\left(\gamma_{1}(m)\right) \iota_{2}\left(\gamma_{2}(s)\right)\right]=\frac{1}{\sqrt{\left(\operatorname{det} Q, m^{k}\right)\left(\operatorname{det} Q, s^{k}\right)}} \sum_{X \in M_{2 k, 2}(\mathbb{Z})} \alpha(X, Q, l, m) \\
e\left(x_{1}^{T} D_{m}\left(V^{T} Q V\right)^{-1} D_{m} x_{1}+x_{1}^{T} D_{m}\left(V^{T} Q V\right)^{-1} D_{s} x_{2}+x_{2}^{T} D_{s}\left(V^{T} Q V\right)^{-1} D_{s} x_{2}\right)
\end{array}
$$

where $|\alpha(X, Q, l, m)|=1$ and $D_{m}, D_{s}$ and $V$ are defined as before.

Proof. If both $r, s \neq 1$, the proof works exactly as in Lemma 65. For $m=1$ or $s=1$ we need to follow another approach as the lower left block matrix of $\iota_{1}\left(\gamma_{1}(m)\right) \iota_{2}\left(\gamma_{2}(s)\right)$ might have determinant 0 . To solve this, we proceed as in Lemma 62, 
We expand

$$
\theta(Q, Z) \mid\left[\iota_{1}\left(\gamma_{1}(m)\right) \iota_{2}\left(\gamma_{2}(s)\right)\right]=\sum_{T} A_{m, s}(T) e\left(\frac{\operatorname{tr} T Z}{\tilde{m} d \tilde{s}}\right)
$$

and estimate

$$
A_{m, s}(T) \leqslant \frac{r\left(\tilde{m} d \tilde{s} D_{\tilde{m} d \tilde{s}} Q_{\tilde{m} d \tilde{s}}^{-1}, T\right)}{\sqrt{\left(\operatorname{det} Q, m^{k}\right)\left(\operatorname{det} Q, s^{k}\right)}} .
$$

By assuming that $T \in \mathcal{R}_{2}$, the contribution of the theta series is bounded by

$$
\sum_{d \tilde{m} \tilde{s}=N} \frac{d^{3}}{\left(\operatorname{det} Q, d^{k}\right)} \tilde{s}^{2} \tilde{m} \sum_{\substack{T \in \mathcal{R} \\ \tilde{s} \tilde{m} \mid \operatorname{det} T \ll N^{2+\epsilon}}} \frac{r\left(N Q^{-1}, T\right)^{2}}{\operatorname{det} Q}\left(\frac{N^{2}}{\operatorname{det} T}\right)^{k-\frac{3}{2}} N^{\epsilon} .
$$

We estimate the $T$ sum by

$$
\sum_{t_{1} t_{4} \ll N^{2+\epsilon}} \frac{r\left(N Q^{-1}, t_{1}\right) r\left(N Q^{-1}, t_{4}\right)}{\left(t_{1} t_{4}\right)^{k-\frac{3}{2}}} \frac{N^{2 k-3}}{\operatorname{det} Q}
$$

and apply Lemma 22, As a consequence, the display above is bounded by $\frac{N^{2 k-1}}{\sqrt{a}}$ and we obtain that display 109 is bounded by

$$
\sum_{d \tilde{m} \tilde{s}=N} \frac{d^{3}}{\left(\operatorname{det} Q, d^{2 k}\right)} \tilde{s}^{2} \tilde{m} \frac{N^{2 k-1+\epsilon}}{\sqrt{a}} \ll \frac{N^{2 k+1+\epsilon}}{\sqrt{a}} .
$$

For the genus theta series we apply (61) and proceed similarly. This gives a bound of size $N^{\epsilon} \operatorname{det} Q$.

Next, we treat the Klingen-Eisenstein series. For this purpose, let $l, m, s$ denote divisors of $N$. Our aim is to bound the Fourier coefficients of

$$
E_{l}\left(f_{l}, Z\right) \mid\left[\iota_{1}\left(\gamma_{1}(m)\right) \iota_{2}\left(\gamma_{1}(s)\right)\right]=\sum_{T} A_{l, m, s}(T) e\left(\frac{\operatorname{tr}(T Z)}{[m, s]}\right) .
$$

To this end, we commence by decomposing $M \in P(N) \Gamma_{0}^{(2)}(N) \iota_{1}\left(\gamma_{1}(r)\right) \iota_{2}\left(\gamma_{1}(s)\right)$. According to Corollary 45 we need to determine congruence conditions such that

$$
\bigsqcup_{\sigma, v, w, \mu} P(N) L\left(\begin{array}{ll}
v & \\
& v^{-T}
\end{array}\right) \iota_{2}(\sigma)\left(\begin{array}{cc}
I & \mu \\
& I
\end{array}\right)\left(\begin{array}{ll}
w^{-1} & \\
& w^{T}
\end{array}\right)\left(\iota_{1}\left(\gamma_{1}(r)\right) \iota_{2}\left(\gamma_{1}(s)\right)\right)^{-1}
$$

is in $\Gamma_{0}^{(2)}(N)$ if and only if these conditions holds. Then, we apply the machinery from Section 4.3.3 and 4.3.4. The first step is to decompose

$$
[l, m, s]=\tilde{l} \tilde{m} \tilde{s} d d_{1} d_{2} d_{3}
$$


into mutually coprime factors with $d=(l, m, s), d_{1}=\frac{(l, m)}{d}, d_{2}=\frac{(l, s)}{d}, d_{3}=\frac{(m, s)}{d}$. Furthermore, we set $r:=\frac{N}{[l, m, s]}$. We determine the congruence conditions for each factor separately. The following display illustrates how these cases are connected to our previous analysis of prime level:

$$
\begin{aligned}
& R(0) \text { case }: \begin{cases}\text { modulo } r & \longrightarrow E\left(f_{1}, Z\right), \\
\text { modulo } \tilde{l} & \longrightarrow E_{N}\left(f_{2}, Z\right),\end{cases} \\
& R(1) \text { case }: \begin{cases}\text { modulo } \tilde{m} & \longrightarrow E\left(f_{1}, Z\right) \mid\left[\iota_{1}(J)\right], \\
\text { modulo } d_{1} & \longrightarrow E_{N}\left(f_{2}, Z\right)\left[\iota_{1}(J)\right],\end{cases} \\
& R(2) \text { case }: \begin{cases}\text { modulo } \tilde{s} & \longrightarrow E\left(f_{1}, Z\right) \mid\left[\iota_{2}(J)\right], \\
\text { modulo } d_{2} & \longrightarrow E_{N}\left(f_{2}, Z\right)\left[\iota_{2}(J)\right],\end{cases} \\
& R(3) \text { case : } \begin{cases}\text { modulo } d_{3} & \longrightarrow E\left(f_{1}, Z\right) \mid\left[J_{4}\right], \\
\operatorname{modulo} d & \longrightarrow E_{N}\left(f_{2}, Z\right)\left[J_{4}\right]\end{cases}
\end{aligned}
$$

Accordingly, we get the following congruence conditions:

$$
\begin{aligned}
& p|r d \rightarrow p| c \text { or } p \mid v_{1}, \\
& p\left|\tilde{l} d_{3} \rightarrow p\right| v_{2} \text { and } p \mid d, \\
& p \mid \tilde{m} d_{2} \rightarrow \text { case }(a),(b),(c) \text { or }(d) \text { modulo } p \text { in Lemma } 48 \\
& p \mid d_{1} \tilde{s} \rightarrow \text { case }(e),(f),(g) \text { or }(h) \text { modulo } p \text { in Lemma } 48
\end{aligned}
$$

Following the line of arguments in Section 4.3.4, we obtain the largest contribution modulo $r d_{3}$ if $r d_{3} \mid c$. Furthermore, modulo $\tilde{m} d_{2}$, case $(d)$ contributes the most while modulo $d_{1} \tilde{s}$ the largest error term is due to case $(h)$. For the sake of simplicity, we only compute the contribution of this main error term. In this case, the following two congruences hold:

$$
c_{1} \equiv 0\left(\bmod r d \tilde{m} d_{2} d_{1} \tilde{s}\right) \quad c_{1} \not \equiv 0\left(\bmod \tilde{l} d_{3}\right) .
$$

To estimate the Petersson inner product, we apply Corollary 66 .

$$
\begin{aligned}
\left(\tilde{l} d_{3}\right)^{-\frac{k}{2}}\left|f_{\tilde{l} d_{1} d_{2} d}\right|\left[\gamma\left(\tilde{l} d_{3}\right)\right] \mid & \ll \frac{\left(d d_{1} d_{2}\right)^{\frac{k}{2}}}{\sqrt{\left(\operatorname{det} Q,\left(\tilde{l} d d_{1} d_{2}\right)^{k}\right)}} \\
& \times\left(\frac{N^{\frac{k}{2}}}{d_{3}^{\frac{k}{2}} \sqrt{\operatorname{det} Q}}+\frac{\left(d d_{1} d_{2}\right)^{\frac{k}{2}} \sqrt{N}}{\sqrt{\left(\operatorname{det} Q,\left(d d_{1} d_{2} d_{3}\right)^{k}\right)} \sqrt[4]{a\left(d d_{1} d_{2} d_{3}\right)}}\right) .
\end{aligned}
$$


Proceeding as in Section 4.3 .4 we infer that $A_{l, r, s}(T)$ is bounded by

$$
\begin{gathered}
(\operatorname{det} T)^{k-\frac{3}{2}}(\min T)^{\frac{1-k}{2}} N^{\epsilon}\left(\frac{d^{\frac{3}{2}-k}(d, T)^{\frac{1}{2}}}{\left(\operatorname{det} Q, d^{k}\right)} \frac{d_{3}^{-k}}{\sqrt{\left(\operatorname{det} Q, d_{3}^{k}\right)}} \frac{\left(d_{1} d_{2}\right)^{2-k}}{\left(\operatorname{det} Q,\left(d_{1} d_{2}\right)^{k}\right)}\right. \\
\times \frac{(\tilde{m} \tilde{s})^{2-\frac{3 k}{2}}}{\sqrt{\left(\operatorname{det} Q,(\tilde{m} \tilde{s})^{k}\right)}} \frac{\tilde{l}^{\frac{k}{2}}}{\left(\operatorname{det} Q, \tilde{l}^{k}\right)} \frac{r^{\frac{3-k}{2}}(r, T)^{\frac{1}{2}}}{\sqrt{\left(\operatorname{det} Q, r^{k}\right)}} \\
\left.+\left(a\left(d d_{1} d_{2} d_{3}\right)\right)^{-\frac{1}{4}} \frac{d^{2-k}(d, T)^{\frac{1}{2}}}{\left(\operatorname{det} Q, d^{k}\right)} \frac{d_{3}^{\frac{1}{2}-k}}{\sqrt{\left(\operatorname{det} Q, d_{3}^{k}\right)}} \frac{\left(d_{1} d_{2}\right)^{\frac{5}{2}-k}}{\left(\operatorname{det} Q,\left(d_{1} d_{2}\right)^{k}\right)}(\tilde{m} \tilde{s})^{\frac{5}{2}-2 k} \frac{r^{2-k}(r, T)^{\frac{1}{2}} \tilde{l}^{\frac{1}{2}}}{\left(\operatorname{det} Q, \tilde{l}^{k}\right)^{\frac{1}{2}}}\right) .
\end{gathered}
$$

By 108$)$ we infer that $A_{l, r, s}(T)$ contributes at most

$$
\begin{aligned}
\left(d d_{3}\right)^{3} \tilde{s} d_{2} \tilde{m} d_{1} \sum_{\# U=\tilde{s} d_{2}} \sum_{T} A_{l, r, s}\left(U T U^{T}\right)^{2} \frac{\left(\tilde{m} \tilde{s} d d_{1} d_{2} d_{3}\right)^{4 k-6}}{(\operatorname{det} T)^{2 k-3}} & \\
& \ll \frac{d^{2 k+2}}{\left(\operatorname{det} Q, d^{k}\right)^{2}} d_{3}^{k-1} \frac{d_{2}^{2 k+1} d_{1}^{2 k}}{\left(\operatorname{det} Q,\left(d_{1} d_{2}\right)^{k}\right)^{2}} \frac{\tilde{s}^{k+2} \tilde{m}^{k+1}}{\left(\operatorname{det} Q,(\tilde{s} \tilde{m})^{k}\right)} \frac{\tilde{l}^{k}}{\left(\operatorname{det} Q, \tilde{l}^{k}\right)^{2}} \frac{r^{4-k}}{\left(\operatorname{det} Q, r^{k}\right)} N^{\epsilon} \\
& +\sqrt{a\left(d d_{1} d_{2} d_{3}\right)} \frac{d^{2+3 k}}{\left(\operatorname{det} Q, d^{k}\right)^{2}} \frac{d_{3}^{2 k}}{\left(\operatorname{det} Q, d_{3}^{2 k}\right)} \frac{d_{2}^{2 k+2} d_{1}^{2 k+1}}{\left(\operatorname{det} Q,\left(d_{1} d_{2}\right)^{2 k}\right)^{2}} \tilde{s}^{2} \tilde{m} \frac{\tilde{l}}{\left(\operatorname{det} Q, \tilde{l}^{2 k}\right)} r^{3-k} N^{\epsilon}
\end{aligned}
$$

In the last step, we use that the $T$ sum runs over $d^{3} d_{3}^{3} \tilde{s} d \tilde{m} d_{1} N^{\epsilon}$ elements and we save one power in $d$ and $d_{3}$ by assuming that $T \in \mathcal{R}_{2}$ and making use of the negative power of $\min T$. To obtain the contribution of all Klingen-Eisenstein series we let $l, r, s$ vary over all divisors of $N$. As $a(N)=\frac{N^{2}}{\tilde{a}}$, this gives a bound of size

$$
\left(\frac{N^{2 k+2} \sqrt{\tilde{a}}}{(\operatorname{det} Q)^{2}}+\frac{N^{2 k-1} \sqrt{\tilde{a}}}{\operatorname{det} Q}+N^{k-1}\right) N^{\epsilon}
$$

The largest error terms occur in the cases $N \mid d$ and $N \mid d_{3}$ which correspond to the $R(3)$ case in Section 4.3.4. Basically, we obtain the same results as in the prime case, the only difference is that we applied a more elaborate technique in the proof of Lemma 57 .

Altogether, we obtain:

Lemma 73. Let $N$ be squarefree and $G(Z)=\theta(Q, Z)-\theta($ gen $Q, Z)-\sum_{l \mid N} E_{l}\left(f_{l}, Z\right)$. Then,

$$
\langle G, G\rangle \ll \frac{N^{2 k+1+\epsilon}}{\sqrt{a}}
$$

Similar to the case of prime level, the main error term is due to contribution of the theta series in the $R(2)$ case. In combination with Lemma 33 and 34, we obtain the following representation result for squarefree level: 
Theorem 74. Let $m \geqslant 12$ with $4 \mid m$. Consider a positive, integral $m \times m$ matrix $Q$ of squarefree level $N$ such that $\operatorname{det} Q$ is a square and $\left(\operatorname{det} Q, N^{k}\right)=\operatorname{det} Q$. For $m=12$ we additionally assume that $2^{5} \nmid \operatorname{det} Q$. A binary quadratic form $2 T>0$ is represented by $Q$ provided that $\frac{1}{2} X^{T} Q X=T$ is soluble over the $p$-adic integers for all $p$ and

$$
\begin{aligned}
& \min T \gg N^{1+\frac{2}{m-3}+\epsilon}+\left(\frac{\sqrt{N} \operatorname{det} Q}{\sqrt{a}}\right)^{\frac{2}{m-3}} N^{\epsilon}, \\
& \operatorname{det} T \gg N^{2+\epsilon}\left(\frac{N^{5}(\operatorname{det} Q)^{2}}{\sqrt{a}}\right)^{\frac{2}{m-5}} .
\end{aligned}
$$

\subsubsection{Quadratic nebentypus}

Recall that $\theta(Q, Z)$ transforms with respect to the quadratic character

$$
\chi\left(\begin{array}{ll}
A & B \\
C & D
\end{array}\right)=\left(\frac{(-1)^{k} \operatorname{det} Q}{\operatorname{det} D}\right) .
$$

In order to drop the condition that $\operatorname{det} Q$ is a square in Theorem 6 and 74 , we need to estimate Klingen-Eisenstein series and Siegel cusp forms that transform with respect to quadratic characters.

We start with the former. Consider a divisor $l$ of the squarefree level $N$. For $f \in S_{k}(N, \chi)$ we define

$$
E_{l}(f, Z, \chi)=l^{k} \sum_{M \in P(N) \backslash \Gamma_{0}(N) \gamma(l)} \bar{\chi}\left(M \gamma(l)^{-1}\right) j(M, l Z) f(\pi(M l Z))
$$

where $\gamma(l)$ is given by (75). This is well defined as long as $\chi(-1)=(-1)^{k}$ since

$\bar{\chi}\left(L M \gamma(l)^{-1}\right) f(\pi(L M Z)) j(L M, Z)^{-k}=\bar{\chi}\left(d_{4}\right) d_{4}^{-k} \chi\left(M \gamma(l)^{-1}\right) f(\pi(M Z)) j(M, Z)^{-k}$

and $d_{4} \in\{ \pm 1\}$. The twisted Klingen-Eisenstein series can be computed as in Section 4.3.3. The only difference is that the exponential sum in Lemma 52 is twisted by $\bar{\chi}(d)$ in the case $N \mid c$ and by

$$
\bar{\chi}\left(v_{2}^{2} b+d_{1} d\right)=\bar{\chi}\left(v_{2}^{2} b-v_{2}^{2} a d \bar{c}\right)=\bar{\chi}\left(-v_{2}^{2} \bar{c}\right)
$$

if $N \nmid c, N \mid v_{1}$. Furthermore, the term in Lemma 53 is twisted by $\bar{\chi}\left(-v_{1}^{2} c\right)$ in .

If $\chi$ is quadratic, then the results of Theorem 56 also apply for $E(f, Z, \chi)$ and $E_{N}(f, Z, \chi)$ with the only difference that we have $N$ instead of $(N, \operatorname{det} T)$. In the case of $N \mid c$ we use that Kloosterman sums twisted by a quadratic character still satisfy the Weil bound. In other words, we have

$$
\sum_{d(\bmod c)}^{\prime} \bar{\chi}(d) e\left(\frac{m d+n \bar{d}}{N}\right) \leqslant \tau(c)(m, n, c)^{\frac{1}{2}} c^{\frac{1}{2}} .
$$


In the case $N \mid v_{1}$ we estimate the exponential sum by $N^{\frac{3}{2}}$ instead of $N^{\frac{1}{2}}\left(N, T[w]_{2}\right)$. This bound is still sufficient for bounds as in Theorem 6 . For $E_{N}(f, Z)$ the twist does not depend on $v_{2}$ or $d$.

Every Siegel cusp form transforming with respect to a character $\chi$ of $\Gamma_{0}^{(2)}(N)$ can be expressed as a linear combination of Poincaré series

$$
P_{Q}(Z, \chi)=\sum_{M \in P_{0}(N) \backslash \Gamma_{0}^{(2)}(N)} \bar{\chi}(M) e(\operatorname{tr}(Q M Z)) j(M, Z)^{-k}
$$

The Fourier coefficients of $P_{Q}(Z, \chi)$ can be evaluated by the Kitaoka-Petersson formula. To this end, one follows the steps of proof in [15] bearing in mind the additional character. This way, [15, Theorem 1.3] can be extended to Siegel cusp form transforming with respect to (quadratic) characters. The author plans to work this out in detail during his time at the University of Bonn. 


\section{References}

[1] Anatolij N. Adrianov, Quadratic forms and Hecke operators, Grundlehren der mathematischen Wissenschaften, vol. 286, Springer-Verlag, 1987.

[2] Mahdi Asgari and Ralf Schmidt, On the adjoint L-function of the p-adic GSp(4), Journal of Number Theory 128 (2008), no. 8, 2340-2358.

[3] A.O.L Atkin and J. Lehner, Hecke operators on $\gamma_{0}(n)$, Mathematische Annalen 185 (1970), 134-160.

[4] Valentin Blomer, Uniform bounds for Fourier coefficients of theta-series with arithmetic applications, Acta Arithmetica 114 (2004), no. 1, 1-21.

[5] _ Ternary quadratic forms, and sums of three squares with restricted variables, CRM Proceedings and Lecture Notes, American Mathematical Society, 2008, pp. 1-17.

[6] _ Spectral summation formula for GSp(4) and moments of spinor Lfunctions, Journal of the European Mathematical Society 21 (2019), 17511774 .

[7] Valentin Blomer and Jörg Brüdern, A three squares theorem with almost primes, Bulletin of the London Mathematical Society 37 (2005), no. 4, 507513.

[8] Valentin Blomer and Farrell Brumley, On the Ramanujan conjecture over number fields, Annals of Mathematics 174 (2011), no. 1, 581-605.

[9] Siegfried Böcherer and Tomoyoshi Ibukiyama, Surjectivity of Siegel $\Phi$-operator for square free level and small weight, Annales de l'Institut Fourier 62 (2012), no. $1,121-144$.

[10] Jörg Brüdern and Etienne Fouvry, Lagrange's four squares theorem with almost prime variables., Journal für die reine und angewandte Mathematik 454 (1994), 59-96.

[11] Jim Brown and Ameya Pitale, Special values of L-functions for SaitoKurokawa lifts, Mathematical Proceedings of the Cambridge Philosophical Society 155 (2013), no. 2, 237-255.

[12] T. D. Browning and R. Dietmann, On the representation of integers by quadratic forms, Proceedings of the London Mathematical Society 96 (2007), no. 2, 389-416.

[13] Yingchun Cai, Gauss's three squares theorem involving almost-primes, Rocky Mountain Journal of Mathematics 42 (2012), no. 4, 1115-1134. 
[14] J.W.S. Cassels, Rational quadratic forms, London Mathematical Society Monographs, vol. 13, Academic Press, 1978.

[15] Masataka Chida, Hidenori Katsurada, and Kohji Matsumoto, On Fourier coefficients of Siegel modular forms of degree two with respect to congruence subgroups, Abhandlungen aus dem Mathematischen Seminar der Universität Hamburg 84 (2011), 31-47.

[16] Martin Dickson, Hecke eigenvalues of Klingen-Eisenstein series of squarefree level, arXiv preprint arXiv:1512.09069, 2015.

[17] Martin Dickson, Ameya Pitale, Abhishek Saha, and Ralf Schmidt, Explicit refinements of Böcherer's conjecture for Siegel modular forms of squarefree level, Journal of the Mathematical Society of Japan, no. 1, 251-301.

[18] Jordan S. Ellenberg and Akshay Venkatesh, Local-global principles for representations of quadratic forms, Inventiones mathematicae 171 (2007), no. 2, $257-279$.

[19] Eberhard Freitag, Siegelsche modulfunktionen, Springer Berlin Heidelberg, 1983.

[20] Masaami Furusawa and Kazuki Morimoto, Refined global Gross-Prasad conjecture on special Bessel periods and Böcherer's conjecture, To appear in Journal of the European Mathematical Society arXiv:1611.05567.

[21] Elena P. Golubeva, A bound for the representability of large numbers by ternary quadratic forms and nonhomogenous Waring equations, Journal of Mathematical Sciences 157 (2009), no. 4, 543-552.

[22] E. Gottschling, Explizite Bestimmung der Randflächen des Fundamentalbereichs der Modulgruppe zweiten Grades, Mathematische Annalen 138 (1959), 103-124.

[23] I. S. Gradshteyn and I. M. Ryzhik, Table of integrals, series, and products, 7th ed., Academic Press,New York, 2007.

[24] Jonathan Hanke, Local densities and explicit bounds for representability by a quadratic form, Duke Mathematical Journal 124 (2004), no. 2, 351-388.

[25] Erich Hecke, Über Modulfunktionen und die Dirichletschen Reihen mit Eulerscher Produktentwicklung, Mathematische Annalen 114 (1937), 1-28, 316 351.

[26] J. Hsia and M. Icaza, Effective version of Tartakowsky's theorem, Acta Arithmetica 89 (1999), no. 3, 235-253. 
[27] Henryk Iwaniec and Emmanuel Kowalski, Analytic number theory, Colloquium Publications, vol. 53, American Mathematical Society, 2004.

[28] Burton W. Jones, The arithmetic theory of quadratic forms, The Carus Mathematical Monographs, vol. 10, Mathematical Association of America, 1950.

[29] Yoshiyuki Kitaoka, Modular forms of degree $n$ and representation by quadratic forms. II, Nagoya Math. J. 87 (1982), 127-146.

[30] _ Fourier coefficients of Siegel cusp forms of degree two, Nagoya Mathematical Journal 93 (1984), 149-171.

[31]__ Lectures on Siegel modular forms and representation by quadratic forms, Tata Institute Lectures on Mathematics and Physics, Springer, 1986.

[32] _ Local densities of quadratic forms, Investigations in Number Theory (Tokyo, Japan), Mathematical Society of Japan, 1988, pp. 433-460.

[33] _ Modular forms of degree $n$ and representation by quadratic forms. $V$, Nagoya Math. J. 111 (1988), 173-179.

[34] _ Fourier coefficients of Klingen's Eisenstein series for principal congruence subgroups of the symplectic group, Japanese Journal of Mathematics 18 (1992), no. 2, 361-402.

[35] _ Arithmetic of quadratic forms, Cambridge Tracts in Mathematics, vol. 106, Cambridge University Press, 1993.

[36] Markus Klein, Verschwindungssätze für Hermitesche Modulformen sowie Siegelsche Modulformen zu den Kongruenzuntergruppen $\Gamma_{0}^{(n)}(N)$ und $\Gamma^{(n)}(N)$, Dissertation, Universität Saarbrücken, 2004.

[37] Helmut Klingen, Zum Darstellungssatz für Siegelsche Modulformen, Mathematische Zeitschrift 102 (1967), no. 1, 30-43.

[38] _ Introductory lecture on Siegel modular forms, Cambridge Studies in Advanced Mathematics, vol. 20, Cambridge University Press, 1990.

[39] Max Koecher, Zur Theorie der Modulformen n-ten Grades. I, Mathematische Zeitschrift 59 (1954), 399 - 416.

[40] Emmanuel Kowalski, Abhishek Saha, and Jacob Tsimerman, Local spectral equidistribution for Siegel modular forms and applications, Compositio Mathematica 148 (2012), 335-384.

[41] M. Manickam, B. Ramakrishnan, and T. C. Vasudevan, On Saito-Kurokawa descent for congruence subgroups, Manuscripta mathematica 81 (1993), 161182. 
[42] Ian Petrow and Matthew P. Young, A generalized cubic moment and the Petersson formula for newforms, Mathematische Annalen 373 (2018), no. 12, 287-353.

[43] Ameya Pitale and Ralf Schmidt, Bessel models for GSp(4): Siegel vectors of squarefree levels (long version), "http://www.math.ou.edu/ rschmidt/ papers/BSI.pdf", 2007.

[44] S. Raghavan, Modular forms of degree $n$ and representation by quadratic forms, Annals of Mathematics 70 (1959), no. 3, 446-477.

[45] Jeremy Rouse, Integers represented by positive-definite quadratic forms and Petersson inner products, Acta Arithmetica 187 (2019), 81-100.

[46] Abishhek Saha and Ralf Schmidt, Yoshida lifts and simultaneous nonvanishing of dihedral twists of modular L-functions, Journal of the London Mathematical Society 88 (2011), 251-270.

[47] Naser T. Sardari, Quadratic forms and semiclassical eigenfunction hypothesis for flat tori, Communications in Mathematical Physics 358 (2018), 895-917.

[48] Ichiro Satake, Compactification des espaces quotients de Siegel, II, Séminaire Henri Cartan 10 (1957/18), no. 2, 1-10, Expose No. 10.

[49] Ralf Schmidt, Iwahori-spherical representations of GSp(4) and Siegel modular forms of degree 2 with square-free level, Journal of the Mathematical Society of Japan 57 (2005), no. 1, 259-293.

[50] _ The Saito-Kurokawa lifting and functoriality, American Journal of Mathematics 127 (2005), 209-240.

[51] _ _ On classical Saito-Kurokawa liftings, Journal für die reine und angewandte Mathematik 604 (2007), 211-236.

[52] _ Packet structure and paramodular forms, Transactions of the American Mathematical Society 370 (2018), no. 5, 3085-3112.

[53] Rainer Schulze-Pillot, Representation of quadratic forms by integral quadratic forms, Quadratic and Higher Degree Forms, Springer New York, 2013, pp. 233-253.

[54] Alok Shukla, Co-dimensions of the spaces of cusp forms for Siegel congruence subgroups in degree two, Pacific Journal of Mathematics 293 (1993), no. 1, 207-244.

[55] Carl Ludwig Siegel, Über die analytische Theorie der quadratischen Formen, Annals of Mathematics 36 (1935), no. 3, 527-600. 
[56] On the theory of indefinite quadratic forms, Annals of Mathematics 45 (1944), no. $3,577$.

[57] R. Styer, Evaluating symplectic Gauss sums and Jacobi symbols, Nagoya Mathematical Journal 95 (1984), 1-23.

[58] W. Tartakowsky, Die Gesamtheit der Zahlen, die durch eine positive quadratische Form $f\left(x_{1}, \ldots, x_{s}\right)(s \geqslant 4)$ darstellbar sind, Izv. Akad. Nauk SSSR 7 (1929), no. 1, 111-121.

[59] Fabian Waibel, Fourier coefficients of half-integral weight cusp forms and Waring's problem, The Ramanujan Journal 47 (2017), no. 1, 185-200.

[60] _ Moments of spinor L-functions and symplectic Kloosterman sums, The Quarterly Journal of Mathematics 70 (2019), no. 4, 1411-1436.

[61] Lynne H. Walling, Some relations on Fourier coefficients of degree 2 Siegel forms of arbitrary level, Journal of Number Theory 180 (2017), 349-359.

[62] G. L. Watson, Integral quadratic forms, Cambridge University Press, New York, vol. 51, Cambridge Tracts in Mathematics and Mathematical Physics, 1960.

[63] Tonghai Yang, An explicit formula for local densities of quadratic forms, Journal of Number Theory 72 (1998), no. 2, 309-356.

[64] _ Local densities of 2-adic quadratic forms, Journal of Number Theory 108 (2004), no. 2, 287-345. 
Göttingen, 14.05.2020

\section{Erklärungen gemäß Promotionsordnung}

Hiermit versichere ich an Eides statt, dass ich die Dissertation mit dem Titel "Arithmetic and analytical aspects of Siegel modular forms" selbstständig und ohne unerlaubte Hilfe angefertigt habe.

Hiermit erkläre ich, dass ich mich an keiner anderen Universität um einen Doktortitel beworben habe.

Fabian Waibel, Weender Str. 48, 37073 Göttingen 